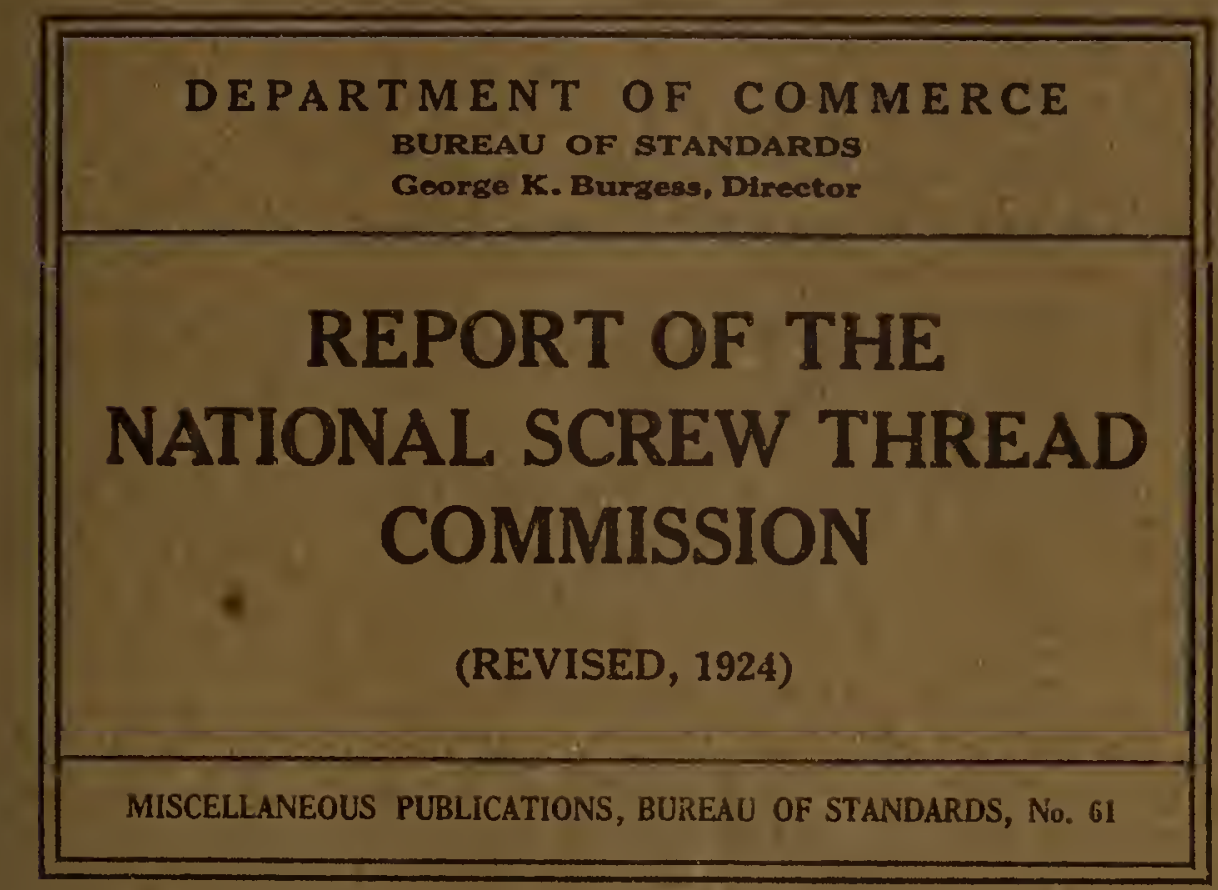



DEPARTMENT OF COMMERCE

BUREAU OF STANDARDS

GEORGE K. BURGESS, DIRECTOR

\section{REPORT}

OF THE

\section{NATIONAL SCREW THREAD COMMISSION}

(REVISED, 1924)

(AUTHORIZED BY CONGRESS, JULY 18, 1918, H. R. 10852)

AS APPROVED AUGUST 19, 1924

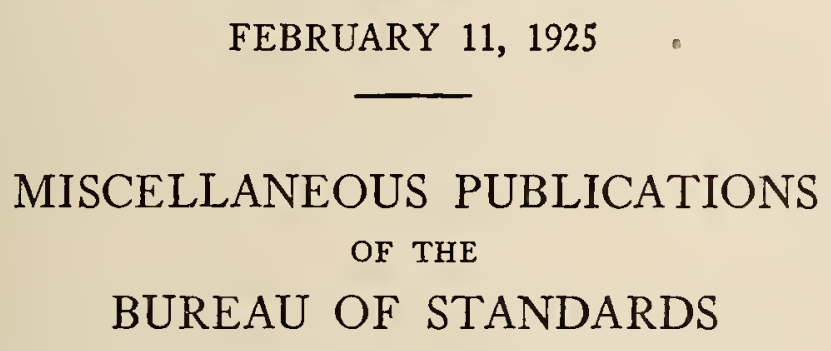

No. 61

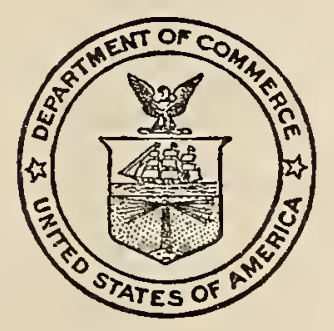

PRICE 35 CENTS

Sold only by the Superintendent of Documents, Government Printing Office Washington, D.C.

WASHINGTON

GOVERNMENT PRINTING OFFICE 


\section{PREFACE}

This report is the first revision of the progress report of the $\mathrm{Na}$ tional Screw Thread Commission published in 1921. The material has been generally rearranged and subdivided into sections, all specifications relating to a given class of product being included in a single section. Among the more important changes made in the specifications as previously published are the following: The classes of fit have been renamed and renumbered; the tolerance on major diameter of screws of classes 3 and 4 has been made the same as for class 2; specifications for gages have been extensively revised, and the allowances and tolerances on fire-hose coupling threads have been revised to decrease the maximum permissible looseness. In general, any screw thread product which met the previous specifications will meet the revised specifications. New material added includes specifications for threading tools, recommended tool shapes, tap dimensions, and tap drill sizes; specifications for screw threads of special diameters, pitches and lengths of engagement; specifications for gages for fire-hose coupling threads; specifications for wood screws; and the appendixes, which embody information supplementing the specifications.

Acknowledgment is made to the many individuals, firms, and other organizations, whose cooperation and assistance have made possible the completion of this report. The thanks of the commission are especially due the manufacturers and users of screw-thread products, tools, and gages; the American Society of Mechanical Engineers; the Society of Automotive Engineers; the American Engineering Standards Committee and its sectional committees on screw threads and plain limit gages; The Federal Specifications Board; the National Board of Fire Underwriters; the United States Army; the United States Nary; and the Bureau of Standards.

Attention is directed to the fact that in so far as the same ground is covered by this revised report of the commission and by report (B 1a-1924) of the American Engineering Standards Committee (Sec. II, Sec. III-1, 2, 3, and 4, and Appendix 1, herein), the two reports are in substantial agreement.

Criticisms and suggestions for the improvement of the report are invited and should be addressed to the National Screw Thread Commission, Bureau of Standards, Washington, D. C. 
APPROVAL BY THE COMMISSION AND TRANSMITTAL TO THE SECRETARIES OF WAR, NAVY, AND COMMERCE

\author{
Hon. John W. Weers, \\ Secretary of War. \\ Hon. Curtes D. Wilber, \\ Secretary of the Navy. \\ Hon. Herbert Hoover, \\ Secretary of Commerce.
}

August 19, 1924.

To the honorables the Secretary of War, the Secretary of the Navy, the Secretary of Commerce.

The National Screw Thread Commission, having revised its Progress Report, dated January 4, 1921, herewith submits its report revised 1924, for your acceptance and approval, in accordance with Public Act No. 201 (H. R. 10852, 65th Cong.), approved July 18, 1918; as amended by Public Act No. 324 (H. R. 15495, 65th Cong.), approved March 3, 1919; Public Resolution No. 34 (H. J. 299, 66th Cong.), approved March 23, 1920; and Public Resolution No. 43 (H. J. 227, 67th Cong.), approved March 21, 1922.

- George K. Burgess,

Chairman.

E. C. Peck, Lieut. Colonel, U. S. A.,

J. O. Johnson, Major, U.S.A., Appointed by the Secretary of War.

M. A. Libbey, Commander, U.S. N., John B. Rirodes, Commander, U.S. N., Appointed by the Secretary of the Navy.

F. O. Wells,

RALPI E. Flanders,

Appointed by the Secretary of Commerce from nominations

by the American Society of Mechanical Engineers.

Earle Buchingham,

George S. Case,

Appointed by the Secretary of Commerce from nominations

by the Society of Automotive Engineers. 
APPROVAL BY THE SECRETARIES OF WAR, NAVY, AND COMMERCE

OCTOBER 2, 1924.

The attached report prepared by the National Screw Thread Commission, in accordance with the law establishing the commission, Public Act No. 201 (H. R. 10852, 65th Cong.), amended by Public Act No. 324 (H. R. 15495, 65th Cong.), is hereby accepted and approved.

JoHN W. WeEKs,

Secretary of War.

Curtis D. Wilbur,

Secretary of the Nary.

Herbert Hoover,

Secretary of Commerce. 


\title{
1924 REPORT OF THE NATIONAL SCREW THREAD COMMISSION
}

\author{
(Authorized by Congress, July 18, 1918, H. R. 10852)
}

AS APPROVED AUGUST 19, 1924

\section{CONTENTS}

Preface

Section

I. Introduction

1. Historical

2. Authorization

(a) Commission authorized by Congress

(b) Life of commission extended by Congress.--

3. Organization of the commission

Page

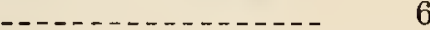

(a) Preliminary meeting

(b) Members

(c) Officers

(d) Committees

(e) Later committees ${ }^{1} \ldots$

$(f)$ Personnel on European trip ${ }^{1}$

(g) Present organization 1

(h) General procedure.................. 10

4. Arrangement of report 1................... 11

5. General

(a) Strict interchangeability ............ 12

(b) Need of definite specifications

Section II. Terminology

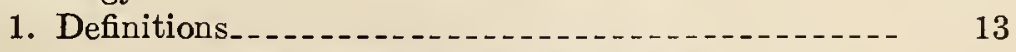

(a) Terms relating to screw threads

(b) Terms relating to classification and toler-

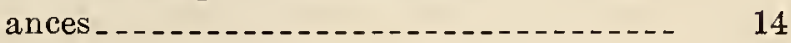

2. Symbols

3. Illustrations showing terminology

Section III. Screw threads for bolts, nuts, commercial tapped holes, etc_- 18

1. National form of thread.

(a) Specifications

(b) Illustration $\ldots \ldots$

2. Thread series._._. 19

(a) National coarse-thread series.......... 19

(b) National fine-thread series._.......... 20

3. Classification and tolerances._._. 21

(a) General specifications.......... 22

(b) Classification of fits.......... 23

4. Tables of dimensions

1 New material not included in the progress report of Jan. 4, 1921. 
Section III. Screw threads for bolts, etc.-Continued.

Page

5. Specifications for threading tools ${ }^{1} \ldots \ldots \ldots \ldots$

(a) Form of tools for producing screws.......

(b) Taps_-....-

(c) Tap-drill sizes

6. Gages ${ }^{1}$

(a) Fundamentals.

(b) Gaging practices and types of gages.......

(c) Specifications for gages................

Section IV. Screw threads of special diameters, pitches, and lengths of engagement ${ }^{1}$

1. Form of thread.

2. Thread series._-_.

3. Classification and tolerances...................

(a) General specifications..........

(b) Classification of fits

4. Specifications for threading tools

(a) Form of tools for producing screws. . .....

(b) Taps_..........

(c) Tap drills

5. Gages.

(a) Specifications for gages-.-.............

Section V. National hose-coupling and fire-hose coupling threads_._..-

1. Form of thread

2. Thread series_..........

(a) National hose-coupling threads..........

(b) National fire-hose coupling threads

3. Allowances and tolerances

4. Tables of dimensions

5. Gages ${ }^{1}$
(a) Gages for national fire-hose coupling threads.

Section VI. National pipe threads.

1. Form of thread.....

(a) Specifications.

(b) Illustration......

2. Symbols

3. Thread series.

(a) National (American Briggs') taper pipe

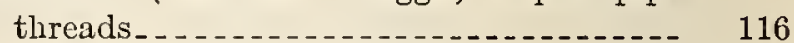

(b) National straight pipe threads. . . . . . . . 120

(c) National locknut threads

4. Tables of pipe dimensions............ 124

5. Threading tools ${ }^{1} \ldots \ldots \ldots$

(a) Taps_...

(b) Tap drills . .

6. Gages _... 128

(a) Classification of gages.............. 128

(b) Gaging practices_..._._._._._._. 130

(c) Specifications for gages_._._._._._._._. 130

Section VII. Wood screws ${ }^{1}$

1. General specifications_._... 139

2. Thread series_._._._... 141

\footnotetext{
1 Now material not included in the progress report of Jan. 4, 1921.
} 
Section VII. Wood screws-Continued. Page

3. Tolerances on length

(a) Flat and oval-head screws._........ 141

(b) Round-head screws._._.

4. Standard sizes of wood screws........ 142

(a) Steel screws

(b) Brass screws....... 142

Appendix 1. Derivation of tolcrances 1

1. Pitch diameter tolerances_....... 145

(a) Tolerances for fastening screws_._._._.- 145

(b) Tolerances for screw threads of special diameters, pitches, and lengths of engage-

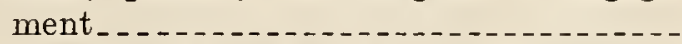

2. Relation of lead and angle errors to pitch diameter

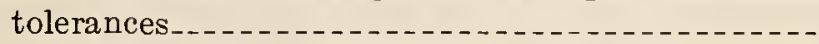

(a) Diameter equivalent of lead error

(b) Diameter equivalent of angle error

Appendix 2. Wire methods of measurcment of pitch diameter 1

1. Size of wires... 147

2. Specification for wires _.................... 149

3. Methods of measuring and using wires_._._._. 149

4. Measurement of pitch diameter of national straight threads

5. Measurement of pitch diameter of national taper

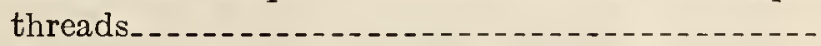

6. Measurement of pitch diameter of thread ring gages

Appendix 3. Control of accuracy of thread elements in the production of thrcaded product ${ }^{1}$

1. Fundamental factors $\ldots \ldots \ldots$

(a) Tool controlled by lead screw

(b) Self-leading threading tool

2. Cutting of screw threads

(a) Single-point tool

(b) Thread chaser..............

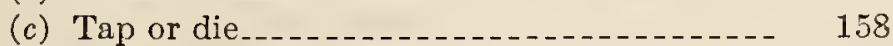

(d) Milling cutter

(e) Threading hob_...... 159

3. Rolling of screw threads_...... 160

(a) Threading roll_....... 160

(b) Thread rolling dies

4. Finishing of screw threads_..................... 161

(a) Grinding $\ldots \ldots 2$

(b) Lapping. 162

Appendix 4. Design and construction of gages.

1. Material

2. Plug thread gages............. 163

(a) Type

(b) Design of "go" thread plug gages _...... 163

(c) Design of "not go" thread plug gages_-.--- 164

3. Ring thread gages....... 164

(a) Type....... 164

(b) Design of "go" thread ring gages_....... 164

(c) Design of "not go" thread ring gages_----- 164

${ }^{1}$ New material not included in tho progress report of Jan. 4, 1921. 
Appendix 4. Design and construction of gages-Continued.

4. Plain plug gages. Page

5. Plain ring gages $\ldots \ldots$

6. Plain snap gages..... 164

Appendix 5. Future work of the commission ${ }^{1} \ldots \ldots \ldots 5$

1. Screw threads used in electrical industry _........ 165

2. Bolt and nut proportions and wrench openings_._._. 165

3. Machine-screw and stove-bolt proportions_._._._. 166

4. Oil-well casing threads

5. Wrench fit of threaded studs

6. Wire gages, and stock sizes of wire, metal sheet, and plate........... 167

7. Acme threads ............. 168

8. Other standardization projects............ 169

(a) Tolerance specifications for pipe threads subject to high pressures........ 169

(b) Instrument-tubing threads_._.......... 169

(c) Threads on instrument screws.......... 169

(d) Form of thread for valve stems........ 169

(e) Threads on condenser-tube ferrules ....... 169

(f) Plumbers' fine threads . . . .

(g) Special threads

Index

\section{SECTION I. INTRODUCTION}

\section{HISTORICAL}

The initial accomplishment in the standardization of screw threads in the United States was the report under date of December 15, 1864, of the special committee appointed by the Franklin Institute on April 21,1861 , for the investigation of a proper system of screw threads, bolt heads, and nuts to be recommended by the institute for adoption and general use by American engineers.

In its report this committee recommended a thread system designed by William Sellers. This thread system specified a single series of pitches for certain diameters from one-fourth inch to 6 inches, inclusive. The threads had an included angle of $60^{\circ}$ and a flat at the crest and root equal to one-eighth of the pitch. This system came into general use and was known as the Franklin Institute thread, the Sellers thread, and commonly as the United States thread.

The accomplishments realized in the adoption of the Franklin Institute, or United States standard thread, in 1864 were brought about largely by the great need of standard threads by American railroads for the development of their lines and equipment. In May, 1868, this thread was adopted by the United States Navy.

In recent years numerous organizations have carried forward the standardization of screw threads. The American Society of Mechani-

1 New material not included in the progress report of Jan. 4, 1921. 
cal Engineers, the Society of Automotive Engineers, the Bureau of Standards, and prominent manufacturers of specialized threaded products have been the chief influences in standardization of screw threads in this country. In England the standardization of screw threads began with the efforts of Sir Joseph Whitworth in ascertaining shop practice in the manufacture of screw threads resulting in the standardization and adoption of the Whitworth thread system, which found extensive use in England. This work has been carried forward by the British Engineering Standards Association, an organization formed in 1901.

While the United States standard thread system fulfilled a great need in the period of the development of our great railway systems, it did not fully meet the requirements of modern manufacture because of the need for additional standard sizes and pitches developed in other industries, and especially because of the need for definitely specified limiting sizes of threaded parts. To fulfill the first of these needs, a thread system having finer pitches than the United States standard system was recommended by the Society of Automotive Engineers, and a machine-screw thread series which provided smaller sizes of screws than the United States standard threads was recommended by the American Society of Mechanical Engineers. The progress of machine design and manufacture has established an extensive use of these fine thread series.

With the great extension of quantity production in this and other countries, particularly during the World War, the need for national standard limiting dimensions was emphasized, as one of the prerequisites of quantity production is standardization of form and dimensions of parts, in order that interchangeability may be established. This is especially important in the matter of screw-thread parts, since there are two mating parts that must fit and these parts in many cases are made in different places. Standardization of screw threads is important to both the manufacturer and the user of a machine, as the user should be able to buy locally a screw or nut for replacement in case of breakage or wear.

\section{AUTHORIZATION}

Through the efforts of several of the engineering societies, the Bureau of Standards, and prominent manufacturers of screw-thread products, a petition was presented to Congress requesting the appointment of a commission to investigate and promulgate standards of screw threads to be adopted by manufacturing plants under the control of the Army and Navy and for adoption and use by the public.

(a) Commission Authorized By Congress.-As a result of this action the National Screw Thread Commission was authorized by 
the following act of Congress, approred July 18, 1918 (Public Act No. 201, H. R. 10852, 65th Cong.):

AN ACT To provide for the appoiniment of a commission to standardize screw threads.

Be it enacted by the Senate and House of Representatives of the United Siates of America in Congress assembled, That a commission is hereby creatcd, to be known as the Commission for the Standardization of Screw Threads, hereinafter referred to as the commission, which shall be composed of nine commissioners, one of whom shall be the Director of the Bureau of Standards, who shall be chairman of the commission; two commissioned officers of the Army, to be appoinied by the Secretary of War; two commissioned officers of the Navy, to be appointed by the Secretary of the Navy; and four to be appointed by the Secretary of Commerce, two of whom shall be chosen from nominations made by the American Society of Mechanical Engineers and two from nominations made by the Society of Automotive Engineers.

SEc. 2. That it shall be the duty of said commission to ascertain and establish standards for screw threads, which shall be submitted to the Secretary of War, the Sccretary of the Navy, and the Secretary of Commerce for their acceptance and approval. Such standards, when thus accepted and approved, shall be adopted and used in the sevcral manufacturing plants under the control of the Nar and Navy Departments, and, so far as practicable, in all specifications for screw threads in proposals for manufactured articles, parts, or materials to be used under the direction of these departments.

SEC. 3. That the Secretary of Commerce shall promulgate such standards for use by the public and cause the same to be published as a public document.

SEc. 4. That the commission shall serve without compensation, but nothing herein shall be held to affect the pay of the commissioners appointed from the Army and Navy or of the Dircetor of the Bureau of Standards.

SEC. 5. That the commission may adopt rules and regulations in regard to its procedure and the conduct of its business.

SEC. 6. That the commission shall cease and terminate at the end of six months from date of its appointment.

Approved, July 18, 1918.

(b) Life of Commission Extended br Congress.-Prior to the expiration of the original term of six months for which the commission was appointed, it became apparent that it would be impossible to complete in a satisfactory manner the work outlined by the commission. Extensions of time were, therefore, asked.by the commission and granted by Congress in accordance with the following acts: Public Act No. 324 (H. R. 15495), 65th Cong.; Public Resolution No. 34 (H. J. Res. 299), 66th Cong.; and Joint Public Resolution No. 43 (H. J. Res. 227), 67th Cong. The term of the commission, as last extended, expires March 21, 1927.

\section{ORGANIZATION OF THE COMMISSION}

(a) Prelminary Meeting.-As soon as nominees were selected by the various organizations to be represented in the commission a preliminary meeting was called at Washington, D. C., on September 12, 1918, by Dr. S. W. Stratton, Director of the Bureau of Standards and chairman of the commission. At this meeting the 
organization of the commission was planned in order that work could be started as soon as formal appointments of the various members of the commission were made. The various commissioners were formally appointed under date of September 21, 1918.

(b) Mevibers. - In accordance with the act, the following members were appointed:

Appointed by the Secretary of Commerce:

Chairman:

Dr. S. W. Stratton, Director of Bureau of StandDate appointed ards, Washington, D. C_._._._.

Dr. G. K. Burgess, Director of Bureau of Standards, Washington, D. C., succeeding Dr. S. W. Stratton - .

On nomination by the American Society of Mechanical

Engineers:

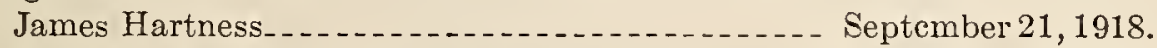

F. O. Wells _. . . . . . . . . . . . . . . . . . September 21, 1918.

Ralph E. Flanders, succeeding James Hartness_..- December 15, 1920.

On nomination by the Society of Automotive Engineers:

H. T. Herr _...

E. H. Ehrman

Earle Buckingham, succeeding H. T. Herr..... April 8, 1921.

George S. Case, succeeding E. H. Ehrman_....... October 3, 1922.

Appointed by the Secretary of War:

E. C. Peck, lieutenant colonel, Ordnance, U. S. Army - - September 21, 1918.

O. B. Zimmerman, major of Engineers, U. S. Army _- September 21, 1918.

John O. Johnson, major of Ordnance, succeeding Miaj.

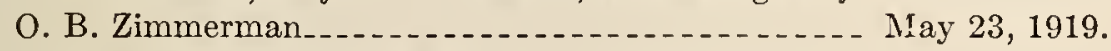

Appointed by the Secretary of the Navy:

E. J. Marquart, commander, U. S. Navy, Bureau of Ordnance _................. September 21, 1918.

S. M. Robinson, commander, U. S. Navy, Bureau of Steam Engineering-......................... September 21, 1918.

N. H. Wright, commander, U. S. Navy, Bureau of Steam Engineering, succeeding Commander S. M. Robinson _. . . . . . . July 14, 1919.

L. N. MeNair, commander, U. S. Navy, Bureau of Ordnance, succeeding Commander E. J. Marquart _ October 7, 1919.

Joseph S. Evans, commander, U. S. Navy, Bureau of Steam Engineering, succeeding Commander N. H.

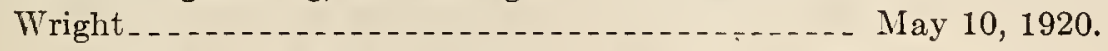

S. M. Robinson, commander, U. S. Navy, Bureau of Steam Engineering, succeeding Commander J. S. Evans. . . . . .

J. N. Ferguson, commander, U. S. Navy, Bureau of Ordnance, succeeding Commander L. N. McNair__._ January 9, 1922.

C. A. Jones, lieutenant commander, U. S. Navy, Bureau of Engineering, succeeding Commander S. M. Robinson ................................... 21, 1922.

M. A. Libbey, commander, U. S. Navy, Bureau of Engineering, succeeding Commander C. A. Jones...- July 19, 1922.

John B. Rhodes, commander, U. S. Navy, Bureau of Ordnance, succeeding Commander J. N. Ferguson _.. February 20, 1924. 
(c) Officers.-The following officers were elected by the commission at the first meeting.

Lieut. Col. E. C. Peck, vice chairman for meetings held in Washington.

James Hartness, vice chairman for meetings held outside of Washington.

H. L. Van Keuren, executive secretary.

H. W. Bearce, general secretary.

Robt. Lacy, first lieutenant of Engineers, U. S. Army, assistant secretary.

A. W. Coombs, stenographic reporter.

(d) Commitrees.-The commission resolved itself into the following subcommittees, with authority to call to their aid one or more experts for counsel. These subcommittees were responsible for compiling and auditing data pertaining to the subject of each committee and for compiling reports for presentation to the commission as a whole, for the action of the commission.

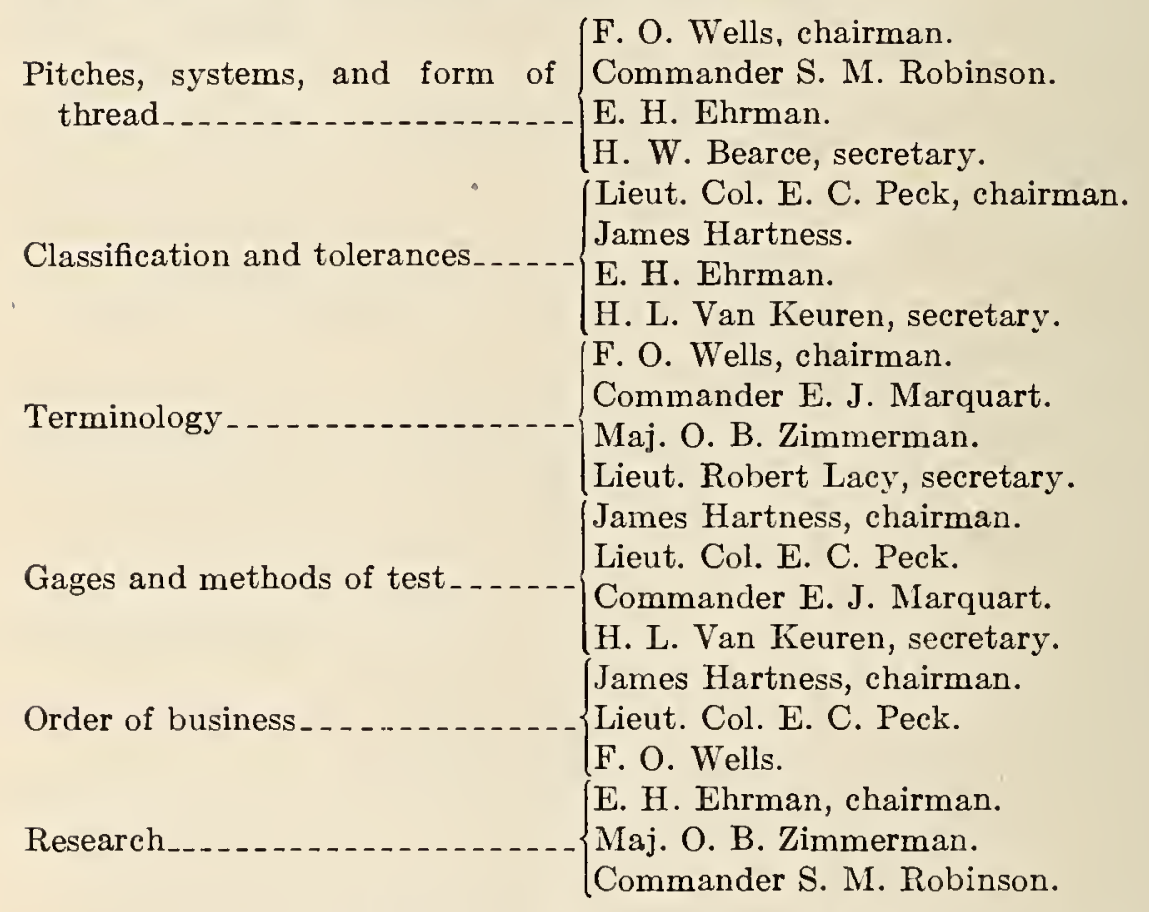

(e) Later Comnttees. - Since the publication of the progress report of 1921 the following additional subcommittees have been appointed:

$\begin{aligned} \text { Taps, dies, tap drills, and wire } & \left\{\begin{array}{l}\text { Lieut. Col. E. C. Peck, chairman. } \\ \text { Ralph E. Flanders. } \\ \text { Earle Buckingham. }\end{array}\right. \\ \text { Boltheads, nuts, and wrenches... } & \left\{\begin{array}{l}\text { F. O. Wells, chairman. } \\ \text { Commander L. N. MeNair (replaced by } \\ \text { Commander J. N. Ferguson). } \\ \text { E. H. Ehrman (replaced by George S. Case). } \\ \text { E. H. Ehrman, chairman (replaced by } \\ \text { George S. Case). } \\ \text { Maj. J. O. Johnson. } \\ \text { Commander Joseph S. Evans (replaced by } \\ \text { Commander M. A. Libbey). }\end{array}\right.\end{aligned}$ 


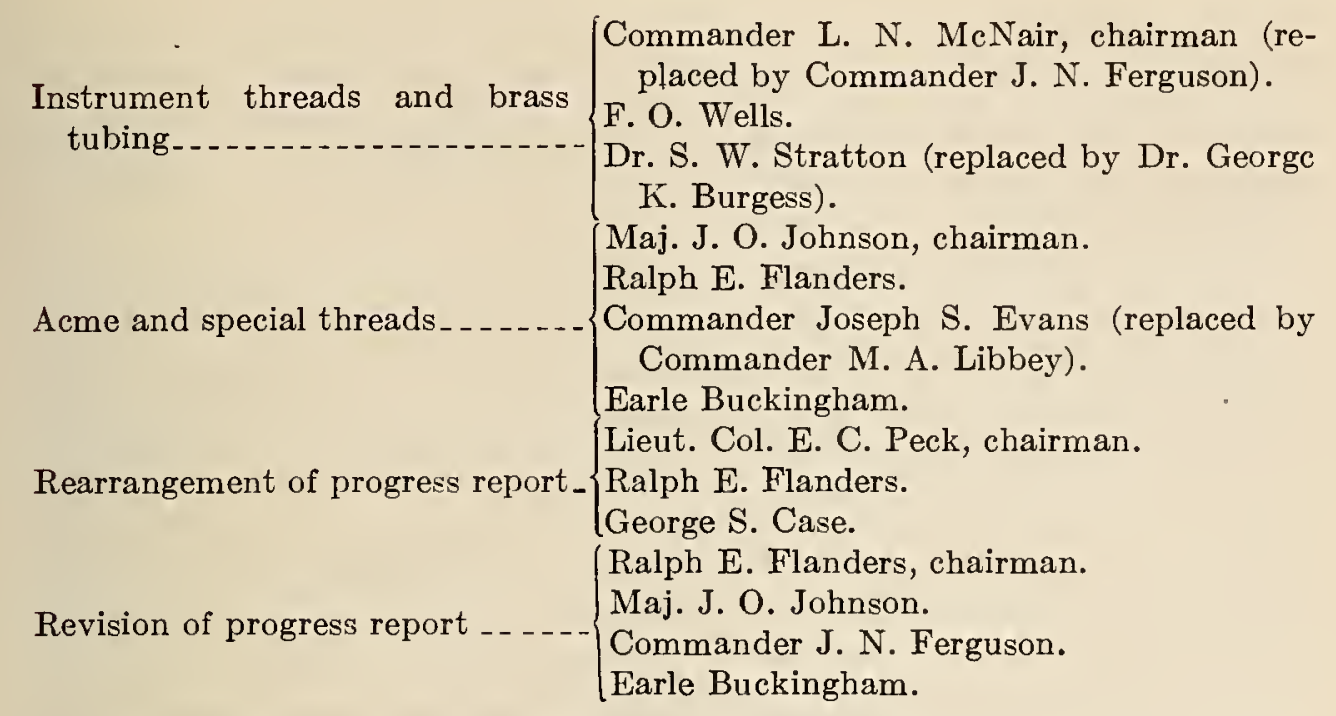

(f) Personnel on European Trip.-In July, 1919, the commission conferred with British and French engineers and manufacturers of screw-thread products, for the purpose of discussing the tentative report prepared by the commission with reference to its suitability to serve as a basis for international standardization of screw threads.

The commission was represented by the following persons:

E. C. Peck (chairman), representative U. S. Army, lieutenant colonel, Ordnance, U. S. Army.

F. O. Wells (vice chairman), representative A. S. M. E.

L. D. Burlingame, representative A. S. M. E., alternate for James Hartness.

E. Buckingham. representative S. A. E., alternate for H. T. Herr.

H. L. Horning, representative S. A. E., alternate for E. H. Ehrman.

J. O. Johnson, representative U. S. Army, major, Ordnance, U. S. Army.

L. B. McBride, representative U. S. Navy, commander, U. S. Navy.

H. C. Dickinson, Representative Department of Commerce, U. S. Government, advisory member.

H. W. Bearce, representative Bureau of Standards, U. S. A. (general secretary).

Robert Lacy, representative U. S. Army, first lieutenant Engineers, U. S. Army (technical secretary).

(g) Present Organization.- At the time of publication of this 1924 revised report the commission comprises the following members:

Dr. George K. Burgess, chairman.

Lieut. Col. E. C. Peck, vice chairman.

F. O. Wells.

Maj. John O. Johnson.

Earle Buckingham.

Ralph E. Flanders.

George S. Case.

Commander John B. Rhodes.

Commander M. A. Libbey.

H. W. Bearce, general secretary.

E. G. Hubbell, stenographic reporter. 
(h) General Procedure.-In its work of establishing standards for screw threads, the commission has made particular efforts to secure actual facts concerning the need of standardization and the economic conditions to be provided for in the production and use of screw threads.

The work of the commission has proceeded rapidly, inasmuch as, in recent years, the accomplishments of the American Society of Mechanical Engincers, the Society of Automotive Engineers, and the British Engincering Standards Association have paved the way toward the adoption of necessary screw-thread standards. Furthermore, the commission has availed itself of the opportunity to secure from the Tap Makers Association and representatives of prominent manufacturing concerns valuable information and data regarding the production of tools and appliances for making threaded products, as well as information and data regarding the application and use of screw-thread products.

Steps were taken to secure from various screw-thread authorities, and representative manufacturers and users, testimony as to the nature of the standards to be adopted for the use of the Government and for American manufacturers. To secure this information public hearings were conducted in various industrial centers throughout the country; and Government officials, authorities on screw threads, manufacturers, and users of screw-thrcad products, as well as manufacturers of taps, dies, gages, and other tools required for producing screw-thread products, were invited to attend these hearings and present their views on rarious phases of the subject. In addition, announcements of the meetings, extending invitations to all interested to be present, were published in the technical magazines. Topic sheets were distributed in adrance of the hearings in order that witnesses could prepare their vicws on the subjects of the meeting in a definite, concise, and authentic form.

A large amount of evidence was collected in this way and the opportunity was available for the various members of the commission to bring out by cross-examination information which could have been secured in no other way. This evidence was tabulated for the consideration of the commission in formulating its report.

A large number of experiments and tests were made by the Bureau of Standards to verify the results obtained at the various hearings and also in connection with the development of tolerances and of other technical subjects considered by the commission. In addition to the experiments conducted by the Bureau of Standards, the members of the commission individually conducted experiments and research work at their own expense.

With regard to the possibilities of international standardization of screw threads, it is the opinion of the commission that not only 
is such international standardization very desirable, but that the present timc is most opportune for accomplishments in this direction. Furthermore, international standardization is of great importance in connection with the development of foreign trade.

In July, 1919, the commission visited Europe to confer with British and French engineering standards organizations, and while no definite agreements were reached in regard to international standardization of screw threads, it was apparent in both France and England that the engineers and manuiacturers in these countries were anxious to cooperate with the United States in this work. The time is very opportune for accomplishments along this line, and it is the opinion of the commission that, as a result of the war, it should be possible to reach an agreement on an international standard thread. Such an international standard should be established by giving consideration to the predominating sizes and standards used in manufactured products, as well as to the possibilities of providing a means for producing this international screw thread by the use of either the English or the metric system of measurement.

The advances made by the commission up to date will facilitate manufacture in case of war, make the best use of labor in our industries in time of peace, increase the safety of travel by rail, steamship, and airplane, and, in general, will increase the dependability of all mechanisms. Every step toward standardization will result in increased production with a minimum expenditure of materials, energies, and other rcsources.

The commission, in formulating this report, has acted largely in a judicial capacity, basing its decisions upon evidence received from authorities on screw-thread subjects and upon the conclusions drawn by other organizations having to do with standardization of screw threads. In addition, the various subjects dealt with havc been considered with a knowledge of present manufacturing conditions and with anticipation of further development in the production of screw-thread products. Above all, it is the intention of the commission to facilitate and promote progress in manufacture.

\section{ARRANGEIMENT OF REPORT}

There are included in the body of the report matters of particular importance and of general interest, while in the appendixes there is arranged supplementary information of both a general and a technical nature. There is included in the body of the report sufficient information to permit the writing of definite and complete specifications for the purchase of screw-thread products.

The specifications in the report have been arranged, as far as possible, by products. For example, one section deals with threads 
for bolts and nuts, etc., another with hose-coupling threads, another with pipe threads, etc. As far as practicable, each section is arranged in the following order:

1. Form of thread.

2. Thread series.

3. Classification and tolerances.

4. Tables of dimensions.

5. Specifications of threading tools.

6. Gages.

\section{GENERAL}

One of the most important phases of standardization of screwthread products is that of interchangeability. The dircct result of establishing a national thread system will bc the elimination of many unnccessary sizes. Ot even morc importance are the advantages to be gained in the manufacture of interchangeable screw-thread parts, especially when made in different manufacturing plants located at a distance from each other, which will assemble without difficulty and in a dependable manner.

(a) Strict Interchangeability.-Many manufacturers, previous to the war, were making interchangeable machine parts in their own shops where there was but one master gage or reference standard, but one individual who had authority to pass on parts in dispute, and where it was possible to secure asscmbly and satisfactory opcration by fitting the parts.

The experience gained by manufacturers producing war material has demonstrated the economic advantage of producing interchangeable parts, especially wherc large quantities of parts are manufactured. In addition to the direct saving in the cost of manufacture, the numerous advantages to be gained make it mandatory that a definite procedure for producing interchangeable work to specified tolcrances be explicitly followed, if we, as manufacturers, arc to keep pace with or lead in the world's progress.

A screw-thread fit can not be accuratcly made with the same facility as the fit of a plain hole and shaft. In the fit of a plain hole and shaft only three clements are taken into account in securing a given class of fit, namcly, rotundity, diamcter, and length; whereas in a screw-thread fit it is necessary to consider rotundity, length, major diameter, pitch diametcr, minor diameter, angle of thread, and pitch or lead. A variation in any one of thcse elements of a screw thread will prevent a perfect fit, so that it is much more difficult to makc a perfect screw-thread fit than it is to make a plain bearing fit.

(b) Need of Definite Specifications.-The difficulties encountered in obtaining enormous quantities of war material needed 
by the United States Government during the recent World War pointed out to Government authorities, as well as manufacturers, the need of writing definite and complete specifications for material required. All specifications should be so written that the qualities in the product desired are stated in definite terms of known measurable standards and correctly defined by the largest tolerance limits compatible with the satisfactory operation or performance of the articles or material for the purpose intended. To this end every factor involved in the acceptability of the manufactured product required should be comparable within specified limits with a known measurable standard. Every specification should be so definite that no dispute regarding the limiting lines of acceptance can arise.

\section{SECTION II. TERMINOLOGY}

In this report there are utilized, as far as possible, nontechnical words and terms which best convey alike to the producer and user of screw threads the information presented.

\section{DEFINITIONS}

The following definitions are given of the more important terms used in the report. Definitions of terms which are obviously elementary in character are intentionally omitted.

(a) Terms Relating to Screw Threads.-1. Screw thread.A ridge of uniform section in the form of a helix on the surface of a cylinder or cone.

2. External and internal threads. - An external thread is a thread on the outside of a member. Example: A threaded plug.

An internal thread is a thread on the inside of a member. Example: A threaded hole.

(These terms are here defined because of possible confusion arising from the fact that an "internal member" has an "external thread," and vice versa.)

3. Major diameter (formerly known as "outside diameter").--The largest diameter of the thread of the screw or nut. The term "major diameter" replaces the term "outside diameter" as applied to the thread of a screw and also the term "full diameter" as applied to the thread of a nut.

4. Minor diameter (formerly known as "core diameter").-The smallest diameter of the thread of the screw or nut. The term "minor diameter" replaces the term "core diameter" as applied to the thread of a screw and also the term "inside diameter" as applied to the thread of a nut.

5. Pitch diameter.-On a straight screw thread, the diameter of an imaginary cylinder, the surface of which would pass through the $16802^{\circ}-25 \dagger-2$ 
threads at such points as to make equal the width of the threads and the width of the spaces cut by the surface of the cylinder. On a taper screw thread, the diameter, at a given distance from a reference plane, of an imaginary cone, the surface of which would pass through the threads at such points as to make equal the width of the threads and the width of the spaces cut by the surface of the cone.

6. Pitch.-The distance from a point on a screw thread to a corresponding point on the next thread measured parallel to the axis.

The pitch in inches $=\frac{1}{\text { Number of threads per inch }}$

7. Lead.-The distance a screw thread advances axially in one turn. On a single-thread screw, the lead and pitch are identical; on a double-thread screw the lead is twice the pitch; on a triplethread screw, the lead is three times the pitch, etc.

8. Angle of thread. - The angle included between the sides of the thread measured in an axial plane.

9. Helix angle.-The angle made by the helix of the thread at the pitch diameter with a plane perpendicular to the axis.

10. Crest.-The top surface joining the two sides of a thread.

11. Root.- The bottom surface joining the sides of two adjacent threads.

12. Side.-The surface of the thread which connects the crest with the root.

13. Axis of a screw.-The longitudinal central line through the screw.

14. Base of thread.-The bottom section of the thread; the greatest section between the two adjacent roots.

15. Depth of thread.-The distance between the crest and the base of thread measured normal to the axis.

16. Number of threads. - Number of threads in 1 inch of length.

17. Length of engagement.-The length of contact between two mating parts, measured axially.

18. Depth of engagement.-The depth of thread contact of two mating parts, measured radially.

(b) Terms Relating to Classification and Tolerances.1. Allowance.-An intentional difference in the dimensions of mating parts. It is the minimum clearance or the maximum interference which is intended between mating parts. It represents the condition of the tightest permissible fit, or the largest internal member mated with the smallest external member. It is to provide for different classes of fit. Examples:

One-half inch, class 1, loose fit, national coarse thread:

Minimum pitch dianeter of nut. 0. 4.500

Maximum pitch diameter of screw _... 4478

Allowance (positive) .0022 
One-half inch, class 4, close fit, national coarse thread:

Minimum pitch diameter of nut_... 0. 4500

Maximum pitch diameter of screw _... . . . . 4504

Allowance (negative)

2. Tolerance.-The amount of variation permitted in the size of a part. Example:

One-half inch screw, class 1, loose fit, national coarse thread:

Maximum pitch diameter.

Minimum pitch diameter._._._. 4404

Tolerance._._. 0074

3. Basic size.--The theoretical or nominal standard size from which all variations are made.

4. Crest clearance.-Defined on a screw form as the space between the crest of a thread and the root of its mating thread.

5. Finish.-The character of the surface on a screw thread.

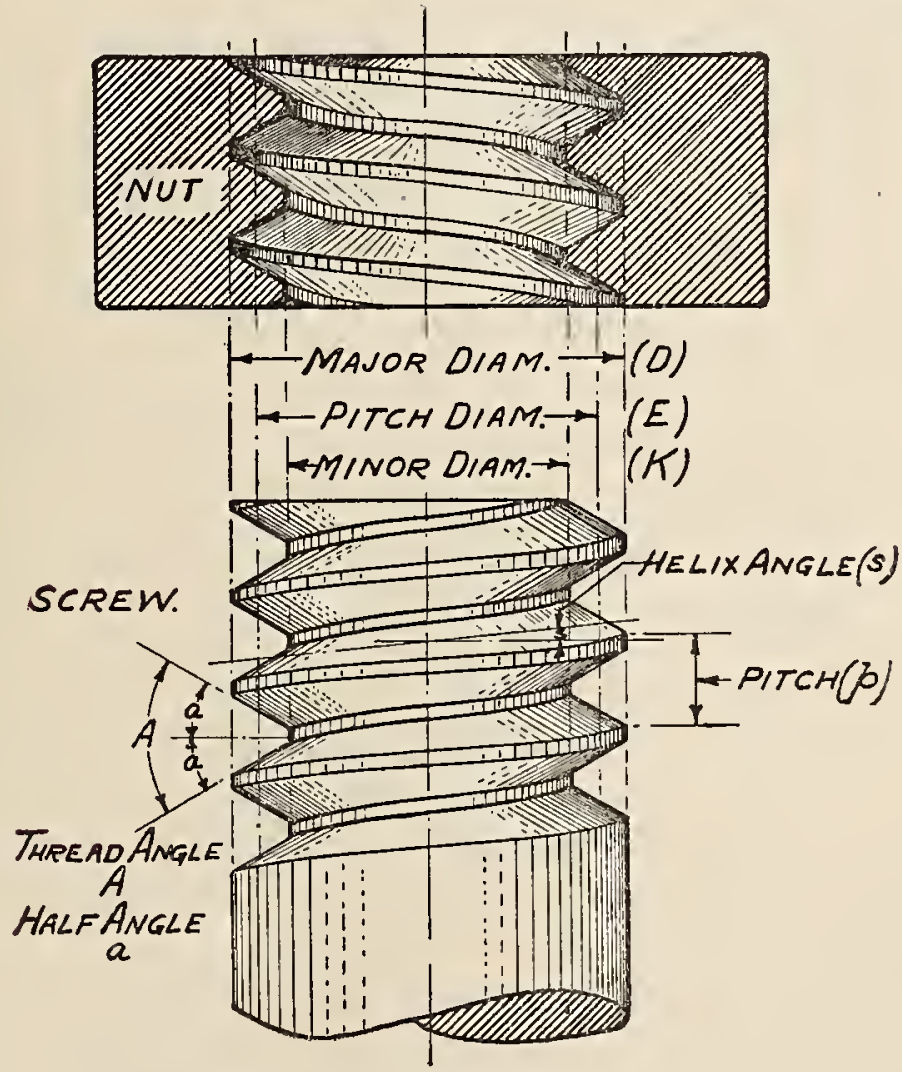

FIG. 1.-Screw thread notation

6. Fit.-The relation between two mating parts with reference to the conditions of assembly; for example: Wrench fit; close fit; medium fit; free fit; loose fit. The quality of fit is dependent upon both the relative size and finish of the mating parts.

7. Neutral zone.-A positive allowance. (See "Allowance.") 
8. Limits.-The extreme permissible dimensions of a part. Example:

One-half inch screw, class 1 , loose fit, national coarse thread:

Maximum pitch diameter.

0.4478 /These are

Minimum pitch diameter. .4404 the limits.

\section{SYMBOLS}

For use in formulas for expressing relations of screw threads, and for use on drawings and for similar purposes, the following symbols should be used:

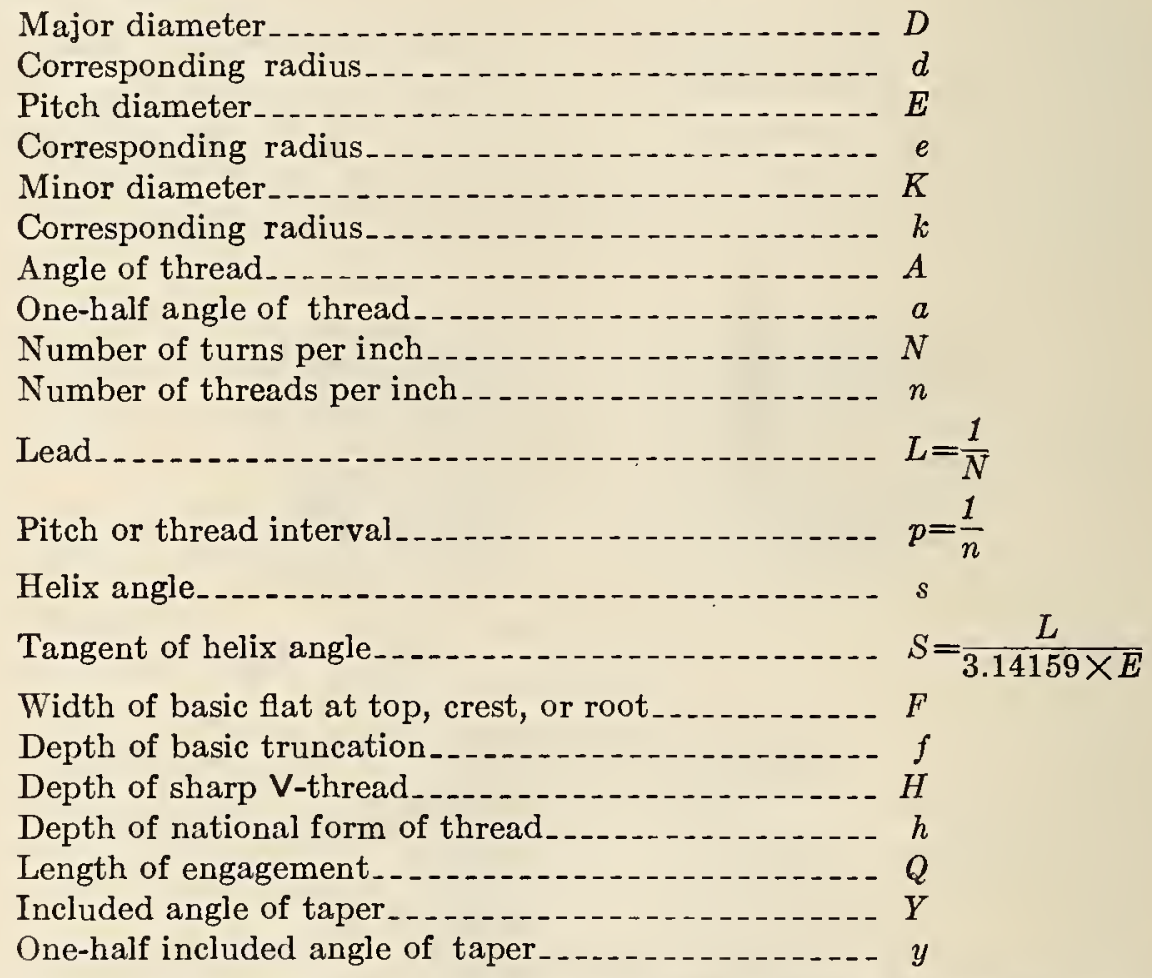

Additional symbols for national pipe threads are given in Section VI.

Symbols are for use on correspondence, drawings, shop and storeroom cards, specifications for parts, taps, dies, gages, etc., and on tools and gages.

The method of designating a screw thread by means of symbols is by the use of the initial letters of the thread series, preceded by the diameter in inches (or the screw number) and number of threads per inch, all in Arabic characters, followed by the classification of fit in Arabic numerals. If the thread is left hand, the symbol "L. H." shall follow the number of threads. No symbol is used to distinguish right-hand threads. Examples: 


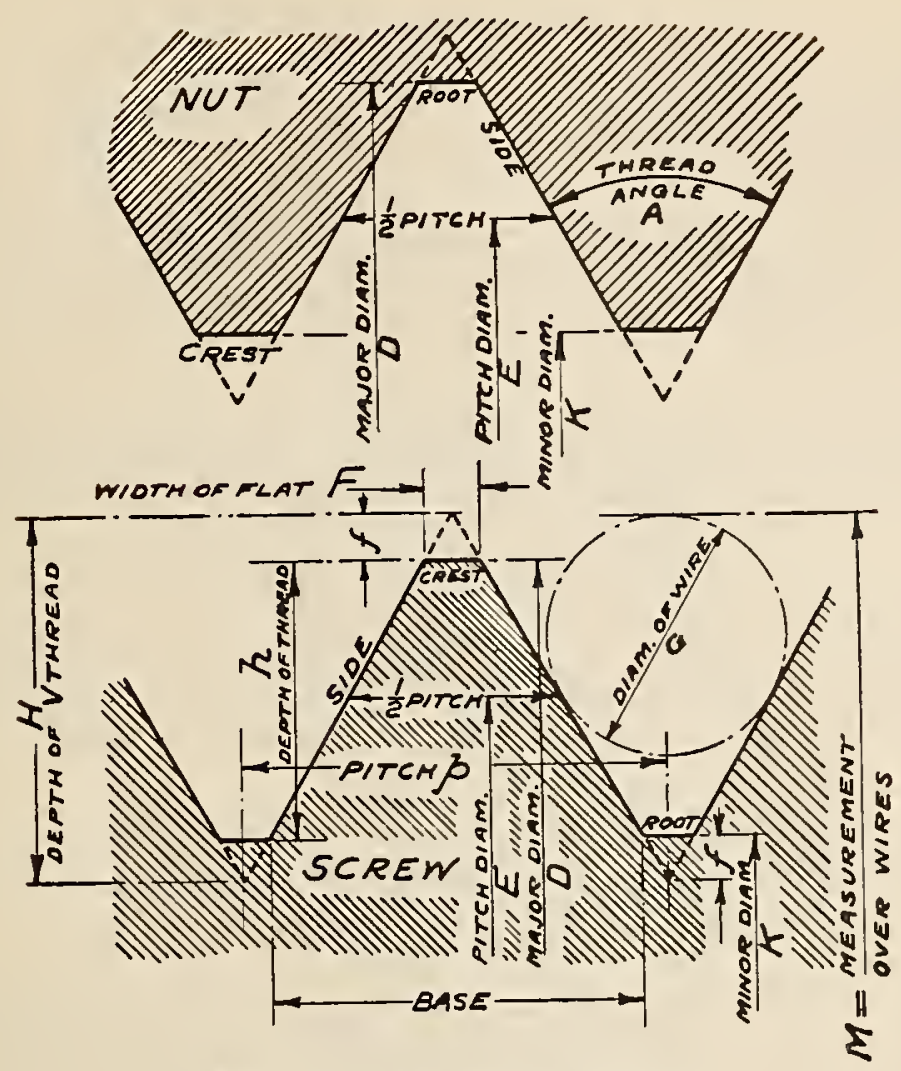

FIG. 2.-Screw thread notation

National Coarse Thread Series:

To specify a threaded part 1 in.-diameter, 8 threads per inch, class 1 fit $1^{\prime \prime}-8-N C-1$

National Fine Thread Series:

Threaded part 1 in.-diameter, 14 threads per inch, class 4 fit.................... $1^{\prime \prime}-14-N F-4$

National Form, Special Pitch:

Threaded part 1 in.-diameter, 12 threads per inch, class 5 fit..................

National Form, Left-Hand Thread:

Threaded part 1 in.-diameter, 12 threads per inch, class 5 fit.................

National Pipe Threads:

National taper pipe thread. Threaded part 1 in.diameter, $11 \frac{1}{2}$ threads per inch $\ldots \ldots \ldots$.

National straight pipe thread . . . . . .

National Fire-Hose Coupling Threads and National Hose-

Coupling Threads:

Threaded part 3 in.-diameter, 6 threads per inch $\ldots$ - $3^{\prime \prime}-6-N H$

Threaded part 1 in.-diameter, 111/2 threads per inch. - $1^{\prime \prime}-111 / 2-N H$

The number of threads per inch must be indicated in all cases, irrespective of whether it is the standard number of threads for that particular size of threaded part or special. 


\section{Symbols for Wire Measurements}

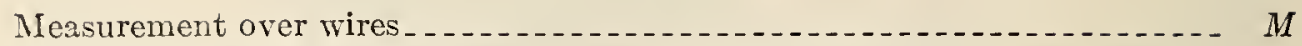

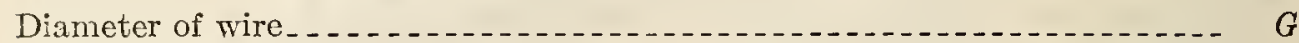

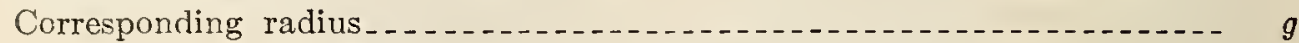

\section{ILLUSTRATIONS SHOWING TERMINOLOGY}

Figures 1 and 2 illustrate the usc of the terms used in the report as previously defined.

\section{SECTION III. SCREW THREADS FOR BOLTS, MACHINE SCREWS, NUTS, COMMERCIAL TAPPED HOLES, ETC.}

\section{NATIONAL FORM OF THREAD}

The form of thread profile specified herein, known previously as the "United States standard or Sellers' profile," is adopted by the commission and shall hereafter be known as the "national form of thread."

The national form of thread shall be used for all screw-thread work cxcept when otherwise specified for special purposes.

\section{(a) SPECIFICATIONS}

1. Axgle of Thread. - The basic angle of thread $(A)$ between the sides of the thread measured in an axial plane is $60^{\circ}$. The line bisecting this $60^{\circ}$ angleis perpendicular to the axis of the screw thread.

2. Flat at Crest and Root. - The flat at the root and crest of the basic thread form is $1 / 8 \times p$, or $0.125 \times p$.

3. Deptu of Thread.-The depth of the basic thread form is

where

$$
h=0.649519 \times p, \text { or } h=\frac{0.649519}{n}
$$

$$
\begin{aligned}
& p=\text { pitch in inches, } \\
& n=\text { number of threads per inch, } \\
& h=\text { basic depth of thread. }
\end{aligned}
$$

4. Clearance at Minor Diameter.-A clearance shall be provided at the minor diameter of the nut by removing from the crest of the basic thread form an amount equal to one-sixth to one-fourth of the basic thread depth.

5. Clearance at Major Diameter.-A clearance at the root of the thread at the major diameter of the nut shall be provided by decreasing the depth of the truncation triangle any desired amount not exceeding two-thirds of its theoretical value.

\section{(b) ILIUSTRATION}

There are indicated in Figure 3 the relations as specified herein for the national form of thread for the minimum nut and maximum screw, frce or medium fits. These relations are further shown in Figures 7 and 9 . 


\section{THREAD SERIES}

It is the aim of the commission, in establishing thread systems for general use, to eliminate all unnecessary sizes and, in addition, to utilize as far as possible present predominating sizes. While from certain standpoints it would have been desirable to make simplifications in the thread systems and to establish more thoroughly consistent standards, it is believed that any radical change at the present time would be out of place and interfere with rnanufacturing conditions, and would involve great economic loss.

The testimony given at the various hearings held by the commission is very consistent in favoring the maintenance of the present coarsethread and fine-thread series, the coarse-thread series being the "United States standard" threads, supplemented in the sizes below one-fourth inch by sizes taken from the standard established by the American Society of Mechanical Engineers (A. S. M. E.). The fine-

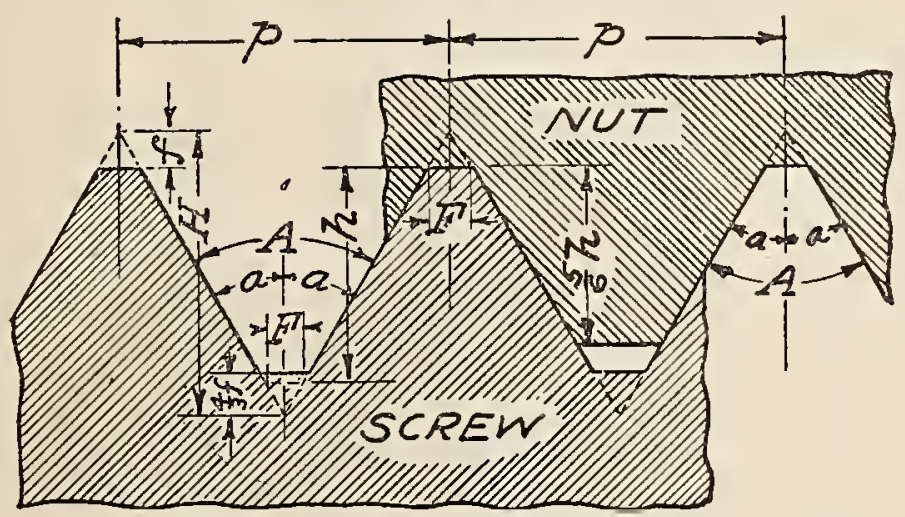

FIG. 3.-National form of thread

Note: No allowance is shown. This condition exists in class 2 , free fit, and class 3 , medium fit, where both the minimum nut and the maximum screw are basic

$$
\text { NOTATION }
$$

$$
\begin{aligned}
& n=\text { number of threads per inch } \\
& \begin{aligned}
n & =\text { number of thrcads per inch } \\
H & =0.866025 \mathrm{p} \text { depth of } 60^{\circ} \text { sharp } \vee \text { thread }
\end{aligned} \\
& h=0.649519 p \text { depth of national form thread } \\
& 8 / 6 h=0.541266 p \text { maximum depth of engagement } \\
& F=0.125000 p \text { width of tlat at crest and root of national form } \\
& f=0.108253 p \\
& \left.\begin{array}{l}
=1 / 8 H \\
=1 / 6 h
\end{array}\right\} \text { depth of truncation }
\end{aligned}
$$

thread series is composed of standards that have been found necessary and consists of sizes taken from from the standards of the Society of Automotive Engineers (S. A. E.), and the fine-thread series of the American Society of Mechanical Engineers (A. S. M. E.).

\section{(a) NATIONAL COARSE-THREAD SERIES}

In Table 1 are specified the nominal sizes and basic dimensions of the "national coarse-thread series."

The national coarse threads are recommended for general use in engineering work, in machine construction where conditions are favorable to the use of bolts, screws, and other threaded components where quick and easy assembly of the parts is desired, and for all work where conditions do not require the use of of fine-pitch threads. 
TABLE 1.-National coarse-thread series

\begin{tabular}{|c|c|c|c|c|c|c|c|c|c|c|c|}
\hline \multicolumn{2}{|c|}{ Identification } & \multicolumn{3}{|c|}{ Basic diameters } & \multicolumn{7}{|c|}{ Thread data } \\
\hline Sizes & $\begin{array}{c}\text { Threads } \\
\text { per } \\
\text { inch } \\
n\end{array}$ & $\begin{array}{c}\text { Major } \\
\text { diame- } \\
\text { ter } \\
D\end{array}$ & $\begin{array}{c}\text { Pitch } \\
\underset{E}{\text { diameter }}\end{array}$ & $\underset{K}{\substack{\text { Minor } \\
\text { diameter }}}$ & $\begin{array}{l}\text { Metric } \\
\text { equiva- } \\
\text { lent of } \\
\text { major } \\
\text { diame- } \\
\text { ter }\end{array}$ & $\underset{p}{\text { Pitch }}$ & $\begin{array}{c}\text { Depth } \\
\text { of } \\
\text { thread } \\
h\end{array}$ & $\begin{array}{c}\text { Basic } \\
\text { width } \\
\text { of fat } \\
p / 8\end{array}$ & $\begin{array}{c}\text { Mini- } \\
\text { mum } \\
\text { width } \\
\text { of flat } \\
\text { at major } \\
\text { diameter } \\
\text { of nut } \\
\text { p/24 }\end{array}$ & $\begin{array}{r}\text { Heli } \\
\text { angl } \\
\text { at ba } \\
\text { pitc } \\
\text { diam } \\
8\end{array}$ & $\begin{array}{l}x \\
\text { e } \\
\text { sic } \\
\text { b } \\
\text { ter }\end{array}$ \\
\hline 1 & 2 & 3 & 4 & 5 & 6 & 7 & 8 & 9 & 10 & 11 & \\
\hline & $\begin{array}{l}64 \\
56 \\
48 \\
40 \\
40\end{array}$ & $\begin{array}{l}\text { Inches } \\
0.073 \\
.086 \\
.099 \\
.112 \\
.125\end{array}$ & $\begin{array}{c}\text { Inches } \\
0.0629 \\
.0744 \\
.0855 \\
.0958 \\
.1088\end{array}$ & $\begin{array}{c}\text { Inches } \\
0.0527 \\
.0628 \\
.0719 \\
.0795 \\
.0925\end{array}$ & $\begin{array}{l}m m \\
1.854 \\
2.184 \\
2.515 \\
2.845 \\
3.175\end{array}$ & $\begin{array}{c}\text { Inch } \\
0.01562 \\
.01786 \\
.02083 \\
.02500 \\
.02500\end{array}$ & $\begin{array}{c}\text { Inch } \\
0.01015 \\
.01160 \\
.01353 \\
.01624 \\
.01624\end{array}$ & $\begin{array}{c}\text { Inch } \\
0.00195 \\
.00223 \\
.00260 \\
.00312 \\
.00312\end{array}$ & $\begin{array}{c}\text { Inch } \\
0.00065 \\
.00074 \\
.00087 \\
.00104 \\
.00104\end{array}$ & $\begin{array}{c}D e g .1 \\
4 \\
4 \\
4 \\
4 \\
4\end{array}$ & $\begin{aligned} \text { Iin. } \\
31 \\
22 \\
26 \\
45 \\
11\end{aligned}$ \\
\hline $\begin{array}{l}6 . \\
8 . \\
10 \\
12\end{array}$ & $\begin{array}{l}32 \\
32 \\
24 \\
24\end{array}$ & $\begin{array}{l}.138 \\
.164 \\
.190 \\
.216\end{array}$ & $\begin{array}{l}.1177 \\
.1437 \\
.1629 \\
.1889\end{array}$ & $\begin{array}{l}.0974 \\
.1234 \\
.1359 \\
.1619\end{array}$ & $\begin{array}{l}3.505 \\
4.166 \\
4.826 \\
5.486\end{array}$ & $\begin{array}{l}.03125 \\
.03125 \\
.04167 \\
.04167\end{array}$ & $\begin{array}{l}.02030 \\
.02030 \\
.02706 \\
.02706\end{array}$ & $\begin{array}{l}.00391 \\
.00391 \\
.00521 \\
.00521\end{array}$ & $\begin{array}{l}.00130 \\
.00130 \\
.00174 \\
.00174\end{array}$ & $\begin{array}{l}4 \\
3 \\
4 \\
4\end{array}$ & $\begin{array}{r}50 \\
58 \\
39 \\
1\end{array}$ \\
\hline & $\begin{array}{l}20 \\
18 \\
16 \\
14 \\
13\end{array}$ & $\begin{array}{l}.2500 \\
.3125 \\
.3750 \\
.4375 \\
.5000\end{array}$ & $\begin{array}{l}.2175 \\
.2764 \\
.3344 \\
.3911 \\
.4500\end{array}$ & $\begin{array}{r}.1850 \\
.2403 \\
.2938 \\
.3447 \\
.4001\end{array}$ & $\begin{array}{r}6.350 \\
7.938 \\
9.525 \\
11.113 \\
12.700\end{array}$ & $\begin{array}{l}.05000 \\
.05556 \\
.06250 \\
.07143 \\
.07692\end{array}$ & $\begin{array}{l}.03248 \\
.03608 \\
.04059 \\
.04639 \\
.04996\end{array}$ & $\begin{array}{l}.00625 \\
.00694 \\
.00781 \\
.00893 \\
.00962\end{array}$ & $\begin{array}{l}.00208 \\
.00231 \\
.00260 \\
.00298 \\
.00321\end{array}$ & $\begin{array}{l}4 \\
3 \\
3 \\
3 \\
3\end{array}$ & $\begin{array}{r}11 \\
40 \\
24 \\
20 \\
7\end{array}$ \\
\hline & $\begin{array}{r}12 \\
11 \\
10 \\
9 \\
8\end{array}$ & $\begin{array}{r}.5625 \\
.6250 \\
.7500 \\
.8750 \\
1.0000\end{array}$ & $\begin{array}{l}.5084 \\
.5660 \\
.6850 \\
.8028 \\
.9188\end{array}$ & $\begin{array}{l}.4542 \\
.5069 \\
.6201 \\
.7307 \\
.8376\end{array}$ & \begin{tabular}{|l|}
14.288 \\
15.875 \\
19.050 \\
22.225 \\
25.400
\end{tabular} & $\begin{array}{l}.08333 \\
.09091 \\
.10000 \\
.11111 \\
.12500\end{array}$ & $\begin{array}{r}.05413 \\
.05905 \\
.06495 \\
.07217 \\
.08119\end{array}$ & $\begin{array}{l}.01042 \\
.01136 \\
.01250 \\
.01389 \\
.01562\end{array}$ & $\begin{array}{l}.00347 \\
.00379 \\
.00417 \\
.00463 \\
.00521\end{array}$ & $\begin{array}{l}2 \\
2 \\
2 \\
2 \\
2\end{array}$ & $\begin{array}{l}59 \\
56 \\
40 \\
31 \\
29\end{array}$ \\
\hline $\begin{array}{l}11 / 8 . \\
11 / 4 \\
11 / 2 . \\
13 / 4 . \\
2 . .\end{array}$ & $\begin{array}{l}7 \\
7 \\
6 \\
5 \\
41 / 2\end{array}$ & $\begin{array}{l}\text { 1. } 1250 \\
1.2500 \\
\text { 1. } 5000 \\
\text { 1. } 7500 \\
2.0000\end{array}$ & $\begin{array}{l}1.0322 \\
1.1572 \\
1.3917 \\
1.6201 \\
1.8557\end{array}$ & $\begin{array}{r}.9394 \\
1.0644 \\
1.2835 \\
1.4902 \\
1.7113\end{array}$ & $\begin{array}{l}28.575 \\
31.750 \\
38.100 \\
44.450 \\
50.800\end{array}$ & $\begin{array}{r}.14286 \\
.14286 \\
.16667 \\
.20000 \\
.22222\end{array}$ & $\begin{array}{r}09279 \\
.09279 \\
.10825 \\
.12990 \\
.14434\end{array}$ & $\begin{array}{r}.01786 \\
.01786 \\
.02083 \\
.02500 \\
.02778\end{array}$ & $\begin{array}{r}00595 \\
.00595 \\
.00694 \\
.00833 \\
.00926\end{array}$ & $\begin{array}{l}2 \\
2 \\
2 \\
2 \\
2\end{array}$ & $\begin{array}{l}31 \\
15 \\
11 \\
15 \\
11\end{array}$ \\
\hline $\begin{array}{l}21 / 4 \\
21 / 2 \\
23 \\
3 .\end{array}$ & $\begin{array}{l}41 / 2 \\
4 \\
4 \\
4\end{array}$ & $\begin{array}{l}2.2500 \\
2.5000 \\
2.7500 \\
3.0000\end{array}$ & $\begin{array}{l}2.1057 \\
2.3376 \\
2.5876 \\
2.8376\end{array}$ & $\begin{array}{l}1.9613 \\
2.1752 \\
2.4252 \\
2.6752\end{array}$ & $\begin{array}{l}57.150 \\
63.500 \\
69.850 \\
76.200\end{array}$ & $\begin{array}{r}.22222 \\
.25000 \\
.25000 \\
.25000\end{array}$ & $\begin{array}{r}.14434 \\
.16238 \\
.16238 \\
.16238\end{array}$ & $\begin{array}{r}.02778 \\
.03125 \\
.03125 \\
.03125\end{array}$ & $\begin{array}{r}.00926 \\
.01042 \\
.01042 \\
.01042\end{array}$ & $\begin{array}{l}1 \\
1 \\
1 \\
1\end{array}$ & $\begin{array}{l}\mathbf{5 5} \\
\mathbf{5 7} \\
\mathbf{4 6} \\
36\end{array}$ \\
\hline
\end{tabular}

(b) NATIONAL FINE-THREAD SERIES

In Table 2 are specified the nominal sizes and basic dimensions of the "national fine-thread series."

The national fine threads are recommended for general use in automotive and aircraft work, for use where the design requires both strength and reduction in weight, and where special conditions require a fine thread, such as, for instance, on large sizes where sufficient force can not be secured by hand to set properly a screw or bolt of coarse pitch. 
TABLE 2.-National fine-thread series

\begin{tabular}{|c|c|c|c|c|c|c|c|c|c|c|}
\hline \multicolumn{2}{|c|}{ Identification } & \multicolumn{3}{|c|}{ Basic diameters } & \multicolumn{6}{|c|}{ Thread data } \\
\hline Sizes & $\begin{array}{c}\text { Threads } \\
\text { per } \\
\text { inch } \\
n\end{array}$ & $\begin{array}{c}\text { Major } \\
\text { diame- } \\
\text { ter } \\
D\end{array}$ & $\begin{array}{c}\text { Pitch } \\
\text { diameter } \\
E\end{array}$ & $\begin{array}{c}\text { Minor } \\
\text { diameter }\end{array}$ & $\begin{array}{c}\text { Metric } \\
\text { equiva- } \\
\text { lentof } \\
\text { major } \\
\text { diame- } \\
\text { ter }\end{array}$ & $\begin{array}{c}\text { Pitch } \\
p\end{array}$ & $\begin{array}{l}\text { Depth } \\
\text { of } \\
\text { thread } \\
h\end{array}$ & $\begin{array}{c}\text { Basic } \\
\text { width } \\
\text { of flat } \\
p / 8\end{array}$ & $\begin{array}{c}\text { Mini- } \\
\text { mum } \\
\text { width } \\
\text { of flat } \\
\text { at major } \\
\text { diameter } \\
\text { of nut } \\
\text { p/24 }\end{array}$ & $\begin{array}{c}\text { Helix } \\
\text { angle } \\
\text { at basic } \\
\text { pitch } \\
\text { diameter } \\
{ }_{8}\end{array}$ \\
\hline 1 & 2 & 3 & 4 & 5 & 6 & y & 8 & 9 & 10 & 11 \\
\hline & $\begin{array}{l}80 \\
72 \\
64 \\
56 \\
48\end{array}$ & $\begin{array}{l}\text { Inches } \\
0.060 \\
.073 \\
.086 \\
.099 \\
.112\end{array}$ & $\begin{array}{c}\text { Inches } \\
0.0519 \\
.0640 \\
.0759 \\
.0874 \\
.0985\end{array}$ & $\begin{array}{r}\text { Inches } \\
0.0438 \\
.0550 \\
.0657 \\
.0758 \\
.0849\end{array}$ & $\begin{array}{l}m m \\
1.524 \\
1.854 \\
\text { 2. } 184 \\
2.515 \\
2.845\end{array}$ & $\begin{array}{c}\text { Inch } \\
0.01250 \\
.01389 \\
.01562 \\
.01786 \\
.02083\end{array}$ & $\begin{array}{c}\text { Inch } \\
0.00812 \\
.00902 \\
.01015 \\
.01160 \\
.01353\end{array}$ & $\begin{array}{l}\text { Inch } \\
0.00156 \\
.00174 \\
.00195 \\
.00223 \\
.00260\end{array}$ & $\begin{array}{c}\text { Inch } \\
0.00052 \\
.00058 \\
.00065 \\
.00074 \\
.00087\end{array}$ & \begin{tabular}{|cc} 
Deg. & Min \\
4 & 2 \\
3 & 5 \\
3 & 4 \\
3 & 4 \\
3 & 5
\end{tabular} \\
\hline & $\begin{array}{l}44 \\
40 \\
36 \\
32 \\
28\end{array}$ & $\begin{array}{l}.125 \\
.138 \\
.164 \\
.190 \\
.216\end{array}$ & $\begin{array}{l}.1102 \\
.1218 \\
.1460 \\
.1697 \\
.1928\end{array}$ & $\begin{array}{r}.0955 \\
.1055 \\
.1279 \\
.1494 \\
.1696\end{array}$ & $\begin{array}{l}\text { 3. } 175 \\
\text { 3. } 505 \\
4.166 \\
4.826 \\
5.486\end{array}$ & $\begin{array}{l}.02273 \\
.02500 \\
.02778 \\
.03125 \\
.03571\end{array}$ & $\begin{array}{l}.01476 \\
.01624 \\
.01804 \\
.02030 \\
.02320\end{array}$ & & & $\begin{array}{l}3 \\
3 \\
3 \\
3 \\
3 \\
3\end{array}$ \\
\hline & $\begin{array}{l}28 \\
24 \\
24 \\
20 \\
20\end{array}$ & $\begin{array}{l}.2500 \\
.3125 \\
.3750 \\
.4375 \\
.5000\end{array}$ & $\begin{array}{l}.2268 \\
.2854 \\
.3479 \\
.4050 \\
.4675\end{array}$ & $\begin{array}{l}.2036 \\
.2584 \\
.3209 \\
.3725 \\
.4350\end{array}$ & $\begin{array}{r}6.350 \\
7.938 \\
9.525 \\
11.113 \\
12.700\end{array}$ & $\begin{array}{l}.03571 \\
.04167 \\
.04167 \\
.05000 \\
.05000\end{array}$ & $\begin{array}{l}.02320 \\
.02706 \\
.02706 \\
.03248 \\
.03248\end{array}$ & & & $\begin{array}{l}2 \\
2 \\
2 \\
2 \\
1\end{array}$ \\
\hline & $\begin{array}{l}18 \\
18 \\
16 \\
14 \\
14\end{array}$ & $\begin{array}{r}.5625 \\
.6250 \\
.7500 \\
.8750 \\
1.0000\end{array}$ & $\begin{array}{l}.5264 \\
.5889 \\
.7094 \\
.8286 \\
.9536\end{array}$ & $\begin{array}{r}.4903 \\
.5528 \\
.6688 \\
.7822 \\
.9072\end{array}$ & $\begin{array}{l}14.288 \\
15.875 \\
19.050 \\
22.225 \\
25.400\end{array}$ & $\begin{array}{l}.05556 \\
.05556 \\
.06250 \\
.07143 \\
.07143\end{array}$ & $\begin{array}{l}.03608 \\
.03608 \\
.04059 \\
.04639 \\
.04639\end{array}$ & $\begin{array}{l}.00694 \\
.00694 \\
.00781 \\
.00893 \\
.00893\end{array}$ & $\begin{array}{l}.00231 \\
.00231 \\
.00260 \\
.00298 \\
.00298\end{array}$ & $\begin{array}{l}1 \\
1 \\
1 \\
1 \\
1\end{array}$ \\
\hline & $\begin{array}{l}12 \\
12 \\
12\end{array}$ & $\begin{array}{l}\text { 1. } 1250 \\
\text { 1. } 2500 \\
\text { 1. } 5000\end{array}$ & $\begin{array}{l}\text { 1. } 0709 \\
1.1959 \\
1.4459\end{array}$ & $\begin{array}{l}1.0167 \\
\text { 1. } 1417 \\
\text { 1. } 3917\end{array}$ & $\begin{array}{l}28.575 \\
31.750 \\
38.100\end{array}$ & $\begin{array}{r}.08333 \\
.08333 \\
.08333\end{array}$ & $\begin{array}{r}.05413 \\
.05413 \\
.05413\end{array}$ & $\begin{array}{l}.01042 \\
.01042 \\
.01042\end{array}$ & $\begin{array}{r}.00347 \\
.00347 \\
.00347\end{array}$ & $\begin{array}{l}1 \\
1 \\
1\end{array}$ \\
\hline
\end{tabular}

\section{CLASSIFICATION AND TOLERANCES}

There are established herein for general use five distinct classes of screw-thread fits as specified in the following brief outline. These five classes of fits, together with the accompanying specifications, are for the purpose of insuring the interchangeable manufacture of screwthread parts throughout the country.

It is not the intention of the commission arbitrarily to place a general class or grade of work in a specific class of fit. Each manufacturer and user of screw threads is free to select the class of fit best adapted to his particular needs. The tolerances and dimensions for four classes of fit are given in Tables 3 to 14, inclusive, and summarized in Tables 15 and 16 . Tolerances and allowances for class 5 , wrench fit, are under consideration by the commission and will be published as soon as determined.

Class 1, loose fit.-... $\left\{\begin{array}{l}\text { Includes screw-thread work of rough com- } \\ \text { mercial quality, where the threads must } \\ \text { assemble readily, and a certain amount } \\ \text { of shake or play is not objectionable. }\end{array}\right.$ 
Class 2, free fit.- $\left\{\begin{array}{l}\text { Includes the great bulk of screw-thread } \\ \text { work of ordinary quality, of finished } \\ \text { and semifinished bolts and nuts, ma- } \\ \text { chine screws, etc. }\end{array}\right.$

Class 3, medium fit................ $\{$ Includes the better grade of interchangeable screw-thread work.

(Includes screw-thread work requiring a

Class 4, close fit............... fine snug fit, somewhat closer than the medium fit. In this class of fit selective assembly of parts may be necessary.

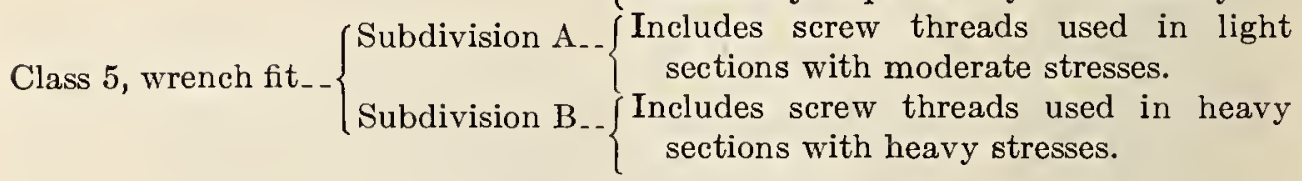

(a) GENERAL SPECIFICATIONS

The following general specifications apply to all classes of fit specified for the "national coarse-thread series" and the "national fine-thread series."

1. Uniform Minimum Nut.-The pitch diameter of the minimum threaded hole or nut corresponds to the basic size, the tolerances being applied above the basic size.

2. Uniform Minor Diameter of Nut.-The minor diameter of the threaded hole or nut, of any given size and pitch, is uniform for fits of classes 1 to 4 , inclusive.

3. Length of Engagement.-A length of engagement equal to the basic major diameter is the basis of the tolerances specified herein for screw-thread products.

4. Tolerances. ${ }^{2}-(a)$ The tolerances specified represent the extreme variations permitted on the product.

(b) The tolerance on the nut is plus, and is applied from the basic size to above basic size.

(c) The tolerance on the screw is minus, and is applied from the maximum screw size to below the maximum screw size.

(d) The pitch diameter tolerances for a screw and nut of a given class of fit are the same.

(e) Pitch diameter tolerances include lead and angle variations. (See footnote 1, Tables 3, 4, 5, and 6.)

(f) The tolerances on the major diameter of class 1 , loose fit, or class 2 , free fit screws are twice the tolerance values allowed on the pitch diameters of the same respective classes, with the exception that on class 2, free fit, "national coarse-thread series," externally threaded parts of unfinished, hot-rolled material, the same tolerances on major diameter are applied as on class 1 , loose fit screws. The tolerances on the major diameters of class 3 , medium fit, and class 4 , close fit screws are the same as those on class 2 , free fit finished screws.

\footnotetext{
${ }^{2}$ Recommendations and explanations regarding the application of tolerances are given in Appendix 1.
} 
(g) The minimum minor diameter of a screw of a given pitch is such as to result in a basic flat. $(1 / 8 \times p)$ at the root when the pitch diameter of the screw is at its minimum value. When the maximum screw is basic, the minimum minor diameter of the screw will be below the basic minor diameter by the amount of the specified pitch diameter tolerance.

(h) The maximum minor diameter of a screw of a given pitch may be such as results from the use of a worn or rounded threading tool, when the pitch diameter is at its maximum value. In no case, however, should the form of a thread, as results from tool wear, be such as to cause the screw to be rejected on the maximum minor diameter by a "go" ring gage, the minor diameter of which is equal to the minimum minor diameter of the nut.

(i) The maximum major diameter of the nut of a given pitch is such as to result in a flat equal to one-third of the basic flat $\left(\frac{1}{24} \times p\right)$ when the pitch diameter of the nut is at its maximum value. When the minimum nut is basic, its maximum major diameter will be above the basic major diameter by the amount of the specified pitch diameter tolerance plus two-ninths of the basic thread depth.

(j) The nominal minimum major diameter of a nut is the basic major diameter. In no case, however, should the minimum major diameter of the nut, as results from a worn tap or cutting tool, be such as to cause the nut to be rejected on the minimum major diameter by a "go" plug gage made to the standard form at the crest.

(k) The tolerance on the minos diameter of a nut of a given pitch is one-sixth of the basic thread depth regardless of the class of fit being produced.

(b) CLASSIFICATION OF FITS

1. Class 1, Loose Fir.-(a) Definition.-The loose-fit class is intended to cover the manufacture of threaded parts where quick and easy assembly is necessary and a considerable amount of shake or play is not objectionable.

This class has an allowance on the screw to permit ready assembly . even when the threads are slightly bruised or dirty.

(b) Minimum nut basic.-- The pitch diameter of the minimum nut of a given diameter and pitch corresponds to the basic pitch diameter, as specified in the tables of thread series given herein, which is computed from the basic major diameter of the thread. The pitch diameter of the minimum nut is the theoretical pitch diameter for that size.

(c) Maximum screw below basic. ${ }^{3}$-The dimensions of the maximum screw of a given pitch and diameter are below the basic dimensions as specified in the tables of thread series given herein, which are

\footnotetext{
3 The maximum minor diameter of the screw is above the basic minor diameter, as shown in Figure 1.
} 
computed from the basic major diameter of the threads, by the amount of the allowance given in Table 3.

(d) Allowance and tolerance values.-Allowances and tolerances are specified in Table 3.

TABLE 3.-Class 1, loose fit, allowances and tolerances for screws and nuts

\begin{tabular}{|c|c|c|c|c|}
\hline Threads per inch & Allowances & $\begin{array}{c}\text { Pitch } \\
\text { diameter } \\
\text { tolerances? }\end{array}$ & $\begin{array}{c}\text { Lead } \\
\text { errors } \\
\text { consuming } \\
\text { one-half } \\
\text { of pitch- } \\
\text { diameter } \\
\text { tolerances } 2\end{array}$ & $\begin{array}{c}\text { Errors in } \\
\text { half-angle } \\
\text { consuming } \\
\text { one-half } \\
\text { of pitch- } \\
\text { diameter } \\
\text { tolerances }\end{array}$ \\
\hline 1 & 2 & 3 & 4 & 5 \\
\hline $\begin{array}{l}80 \\
72+2 \\
64 \\
56 \\
48\end{array}$ & $\begin{array}{l}\text { Inch } \\
0.0007 \\
.0007 \\
.0007 \\
.0008 \\
.0009\end{array}$ & $\begin{array}{l}\text { Inch } \\
0.0024 \\
.0025 \\
.0026 \\
.0028 \\
.0031\end{array}$ & $\begin{array}{c}\text { Inch } \\
0.0007 \\
.0007 \\
.0008 \\
.0008 \\
.0009\end{array}$ & $\begin{array}{rr}\text { Deg. } & \text { Min. } \\
3 & 40 \\
3 & 26 \\
3 & 10 \\
3 & 0 \\
2 & 50\end{array}$ \\
\hline $\begin{array}{l}44 \\
40 \\
36 \\
32 \\
28\end{array}$ & $\begin{array}{l}.0009 \\
.0010 \\
.0011 \\
.0011 \\
.0012\end{array}$ & $\begin{array}{l}.0032 \\
.0034 \\
.0036 \\
.0038 \\
.0043\end{array}$ & $\begin{array}{l}.0009 \\
.0010 \\
.0010 \\
.0011 \\
.0012\end{array}$ & $\begin{array}{ll}2 & 41 \\
2 & 36 \\
2 & 28 \\
2 & 19 \\
2 & 18\end{array}$ \\
\hline $\begin{array}{l}24 \\
18 \\
16 \\
14 \\
14-\end{array}$ & $\begin{array}{l}.0013 \\
.0015 \\
.0016 \\
.0018 \\
.0021\end{array}$ & $\begin{array}{l}.0046 \\
.0051 \\
.0057 \\
.0063 \\
.0070\end{array}$ & $\begin{array}{l}.0013 \\
.0015 \\
.0016 \\
.0018 \\
.0020\end{array}$ & $\begin{array}{l}2 \\
1 \\
1 \\
1 \\
1\end{array}$ \\
\hline $\begin{array}{l}13 \\
12 \\
110 \\
9\end{array}$ & $\begin{array}{l}.0022 \\
.0024 \\
.0026 \\
.0028 \\
.0031\end{array}$ & $\begin{array}{l}.0074 \\
.0079 \\
.0085 \\
.0092 \\
.0100\end{array}$ & $\begin{array}{l}.0021 \\
.0023 \\
.0025 \\
.0027 \\
.0029\end{array}$ & $\begin{array}{l}50 \\
49 \\
47 \\
45 \\
43\end{array}$ \\
\hline $\begin{array}{l}8 \\
7 \\
7 \\
6 \\
51 / 2-1\end{array}$ & $\begin{array}{l}.0034 \\
.0039 \\
.0044 \\
.0052 \\
.0057\end{array}$ & $\begin{array}{l}.0111 \\
.0124 \\
.0145 \\
.0169 \\
.0184\end{array}$ & $\begin{array}{l}.0032 \\
.0036 \\
.0042 \\
.0049 \\
.0053\end{array}$ & $\begin{array}{l}42 \\
39 \\
40 \\
37 \\
35\end{array}$ \\
\hline & .0064 & .0204 & .0059 & 1 \\
\hline
\end{tabular}

1 The tolerances specified for pitch diameter include all errors of pitch diameter, lead, and angle. The full tolerance can not, therefore, be used on pitch diameter unless the lead and angle of the thread are perfect. Columns 4 and 5 give, for information, the errors in lead (per length of thread engaged) and in angle, each of which can be compensated for by half the tolerance on the pitch diameter given in column 3. If lead and angle errors both exist to the amount tabulated, the pitch diameter of a bolt, for example, must be reduced by the full tolerance or it will not enter a basic nut or gage.

2 Between any two threads not farther apart than the length of engagement. 


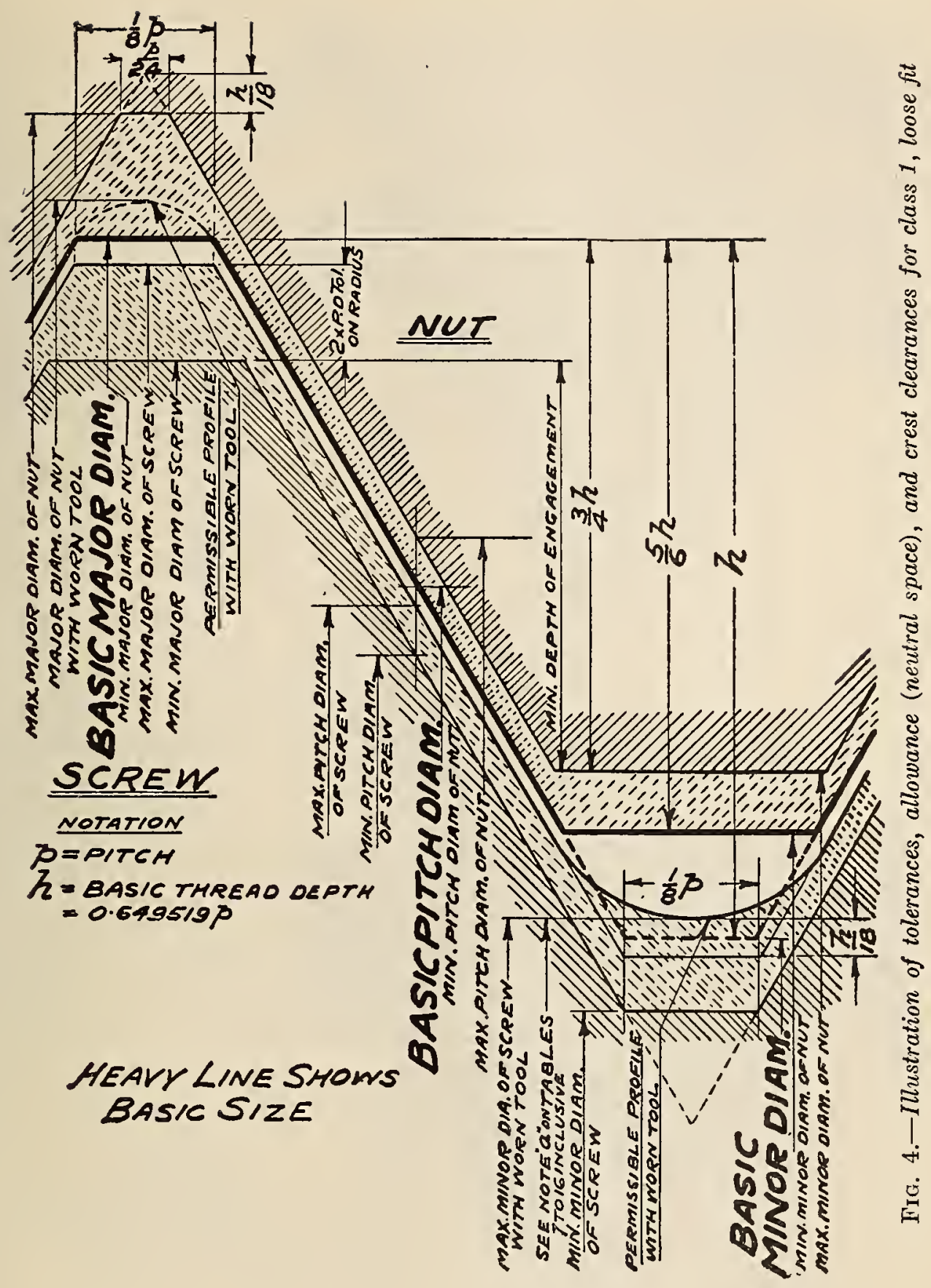




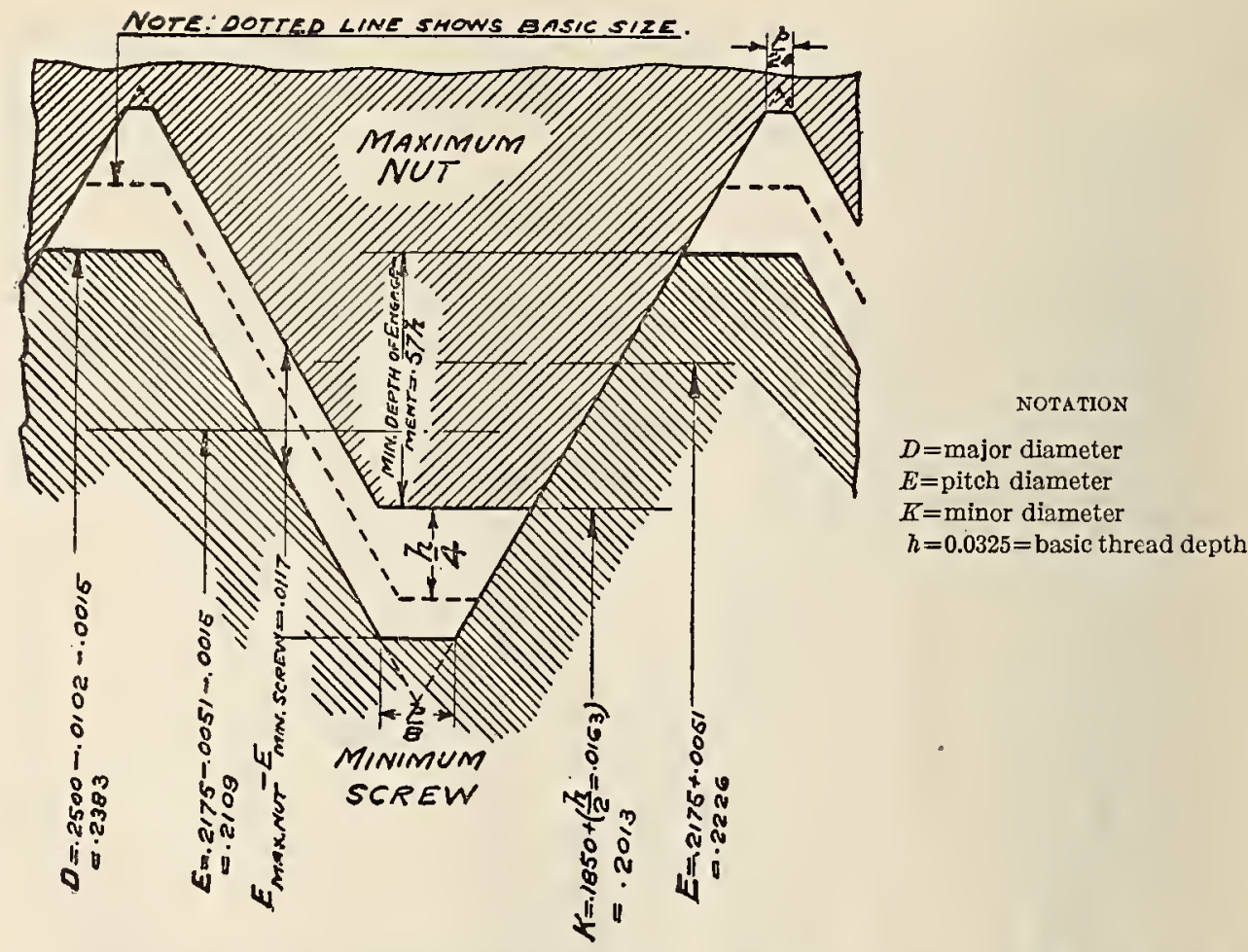

Fig. 5.-Illustration of loosest condition for class 1, loose fit, one-fourth inch, 20 threads

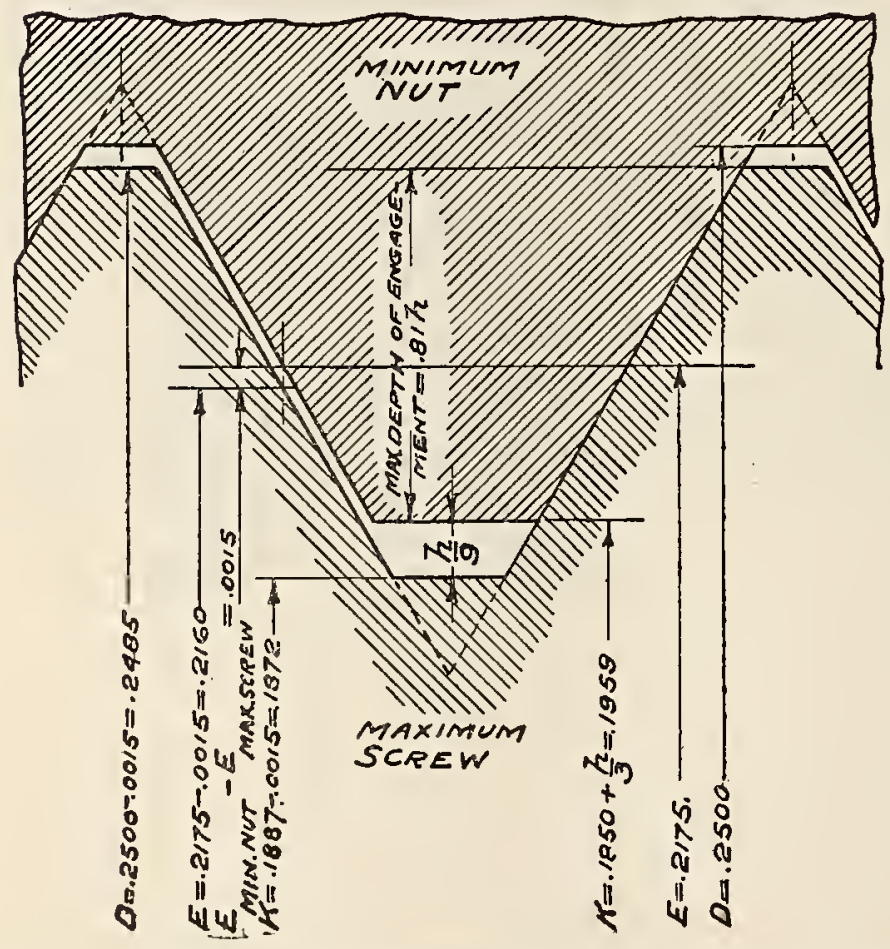

NOTATION

$D=$ major diameter $E=$ nitch diameter $K=$ minor diameter $h=0.0325=$ basic thread depth

FIG. 6.-Illustration of tightest condition for class 1, loose fit, one-fourth inch, 20 threads 
2. Class 2, Free Fit.-(a) Definition.-The free-fit class is intended to apply to interchangeable manufacture where the threaded members are to assemble nearly, or entirely, with the fingers, where

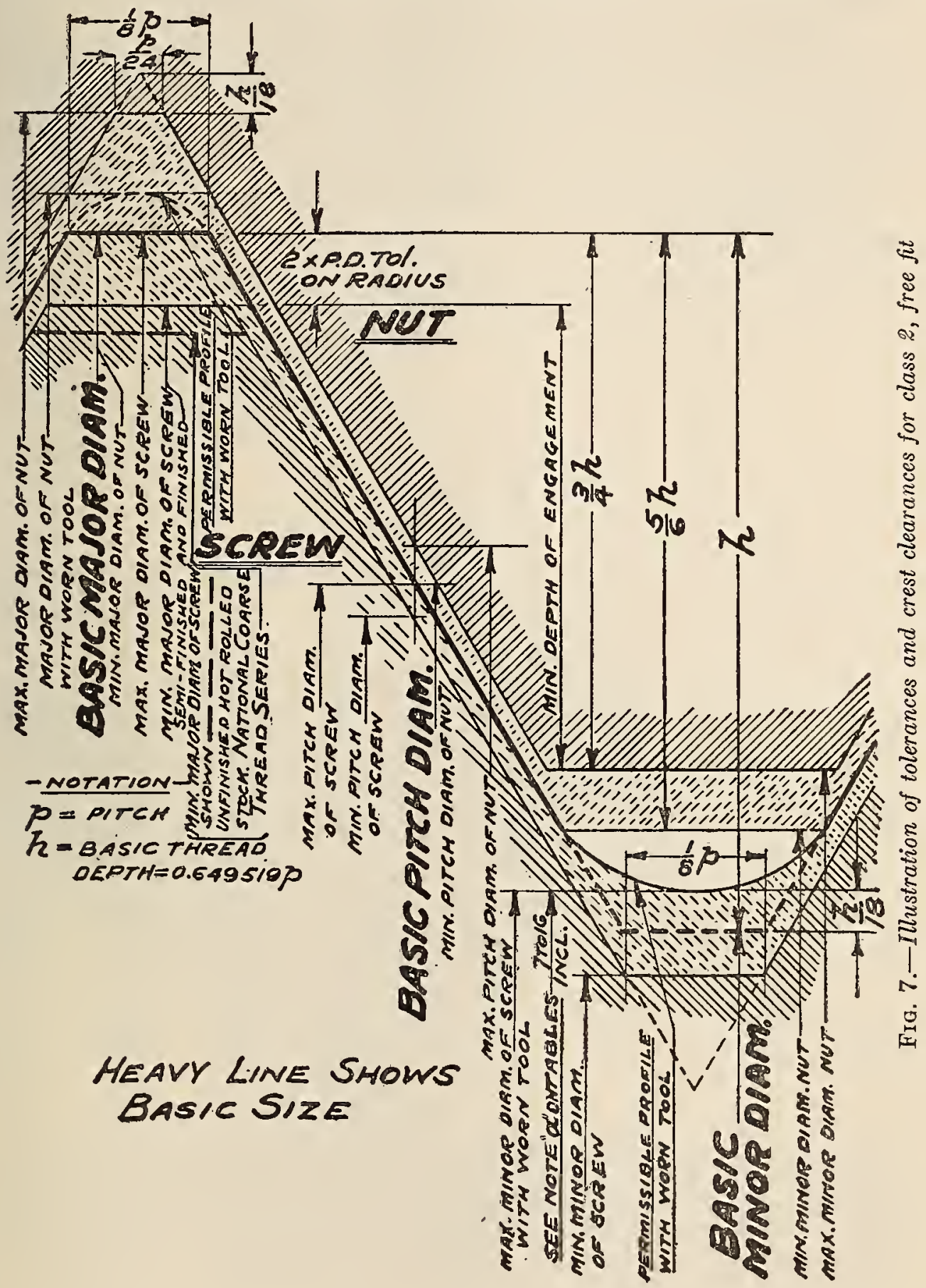

a moderate amount of shake or play between the assembled threaded members is not objectionable, and where no allowance is required. This class includes the great bulk of fastening screws. 
(b) Minimum nut basic.-The pitch diameter of the minimum nut of a given diameter and pitch corresponds to the basic pitch diameter, as specified in tables of thread series given herein, which is computed from the basic major diameter of the thread.

(c) Maximum screw basic. ${ }^{4}$-The major diameter and pitch diameter of the maximum screw of a given pitch and diameter correspond to the basic dimensions, as specified in tables of thread series given herein, which are computed from the basic major diameter of the thread.

(d) Allowance and tolerance values.-Allowances and tolerances are specified in Table 4.

TABLE 4.-Class 2, free fit, allowances and tolerances for screws and nuts

\begin{tabular}{|c|c|c|c|c|c|}
\hline Threads per inch & Allowances & $\begin{array}{c}\text { Pitch } \\
\text { diameter } \\
\text { tolerances }\end{array}$ & $\begin{array}{c}\text { Lead } \\
\text { crrors } \\
\text { consuming } \\
\text { one-half } \\
\text { of pitch- } \\
\text { diameter } \\
\text { tolerances }\end{array}$ & \multicolumn{2}{|c|}{$\begin{array}{l}\text { Errors in } \\
\text { half-angle } \\
\text { consuming } \\
\text { one-half } \\
\text { of pitch- } \\
\text { diameter } \\
\text { tolerances }\end{array}$} \\
\hline 1 & 2 & 3 & 4 & \multicolumn{2}{|l|}{$\bar{j}$} \\
\hline $\begin{array}{ll}80 \\
64 \\
66 \\
48 \\
48 \\
\end{array}$ & $\begin{array}{r}\text { Inch } \\
0.0000 \\
.0000 \\
.0000 \\
.0000 \\
.0000\end{array}$ & $\begin{array}{r}\text { Inch } \\
0.0017 \\
.0018 \\
.0019 \\
.0020 \\
.0022\end{array}$ & $\begin{array}{l}\text { Inch } \\
0.0005 \\
.0005 \\
.0005 \\
.0006 \\
.0006\end{array}$ & \multicolumn{2}{|c|}{$\begin{array}{rr}\text { Deg. } & \text { Min. } \\
2 & 36 \\
2 & 28 \\
2 & 19 \\
2 & 8 \\
2 & 1\end{array}$} \\
\hline $\begin{array}{l}44 \\
40 \\
36 \\
36\end{array}$ & $\begin{array}{l}.0000 \\
.0000 \\
.0000 \\
.0000 \\
.0000\end{array}$ & $\begin{array}{l}.0023 \\
.0024 \\
.0025 \\
.0027 \\
.0031\end{array}$ & $\begin{array}{l}.0007 \\
.0007 \\
.0007 \\
.0008 \\
.0009\end{array}$ & $\begin{array}{l}1 \\
1 \\
1 \\
1 \\
1\end{array}$ & $\begin{array}{l}56 \\
50 \\
43 \\
39 \\
39\end{array}$ \\
\hline $\begin{array}{l}24 \\
20 \\
18\end{array} 16$ & $\begin{array}{l}.0000 \\
.0000 \\
.0000 \\
.0000 \\
.0000\end{array}$ & $\begin{array}{l}.0033 \\
.0036 \\
.0041 \\
.0045 \\
.0049\end{array}$ & $\begin{array}{l}.0010 \\
.0010 \\
.0012 \\
.0013 \\
.0014\end{array}$ & $\begin{array}{l}1 \\
1 \\
1 \\
1 \\
1\end{array}$ & $\begin{array}{l}31 \\
22 \\
25 \\
22 \\
19\end{array}$ \\
\hline $\begin{array}{l}13 \\
12 \\
11 \\
10 \\
10\end{array}$ & $\begin{array}{l}.0000 \\
.0000 \\
.0000 \\
.0000 \\
.0000\end{array}$ & $\begin{array}{l}.0052 \\
.0056 \\
.0059 \\
.0064 \\
.0070\end{array}$ & $\begin{array}{l}.0015 \\
.0016 \\
.0017 \\
.0018 \\
.0020\end{array}$ & $\begin{array}{l}1 \\
1 \\
1 \\
1 \\
1\end{array}$ & $\begin{array}{l}17 \\
17 \\
11 \\
13 \\
12\end{array}$ \\
\hline $\begin{array}{l}8 \\
6 \\
6\end{array}$ & $\begin{array}{l}.0000 \\
.0000 \\
.0000 \\
.0000 \\
.0000\end{array}$ & $\begin{array}{l}.0076 \\
.0085 \\
.0101 \\
.0116 \\
.0127\end{array}$ & $\begin{array}{l}.0022 \\
.0025 \\
.0029 \\
.0033 \\
.0037\end{array}$ & $\begin{array}{l}1 \\
1 \\
1 \\
1 \\
1\end{array}$ & $\begin{array}{r}10 \\
8 \\
9 \\
6 \\
5\end{array}$ \\
\hline $4 \ldots \ldots$ & .0000 & .0140 & .0040 & 1 & 4 \\
\hline
\end{tabular}

- The tolerances specified for pitch diameter include all errors of pitch diameter, lead, and angle. The full tolerance can not, therefore, be used on pitch diameter unless tbe lead and angle of the thread are perfect. Columns 4 and 5 give, for information, the errors in lead (per length of thread engaged) and in angle, each of which can be compensated for by half the tolerance on the pitch diameter given in column 3 . lf lead and angle errors both exist to the amount tabulated, the pitch diamerer of a bolt, for example, must be rcduced by the full tolerance or it will not enter a basic nut or gage.

B Between any two threads not farther apart than the length of engagement.

- The maximum minor diameter of the screw is above the basic minor diameter, as shown in Figure 7 . 


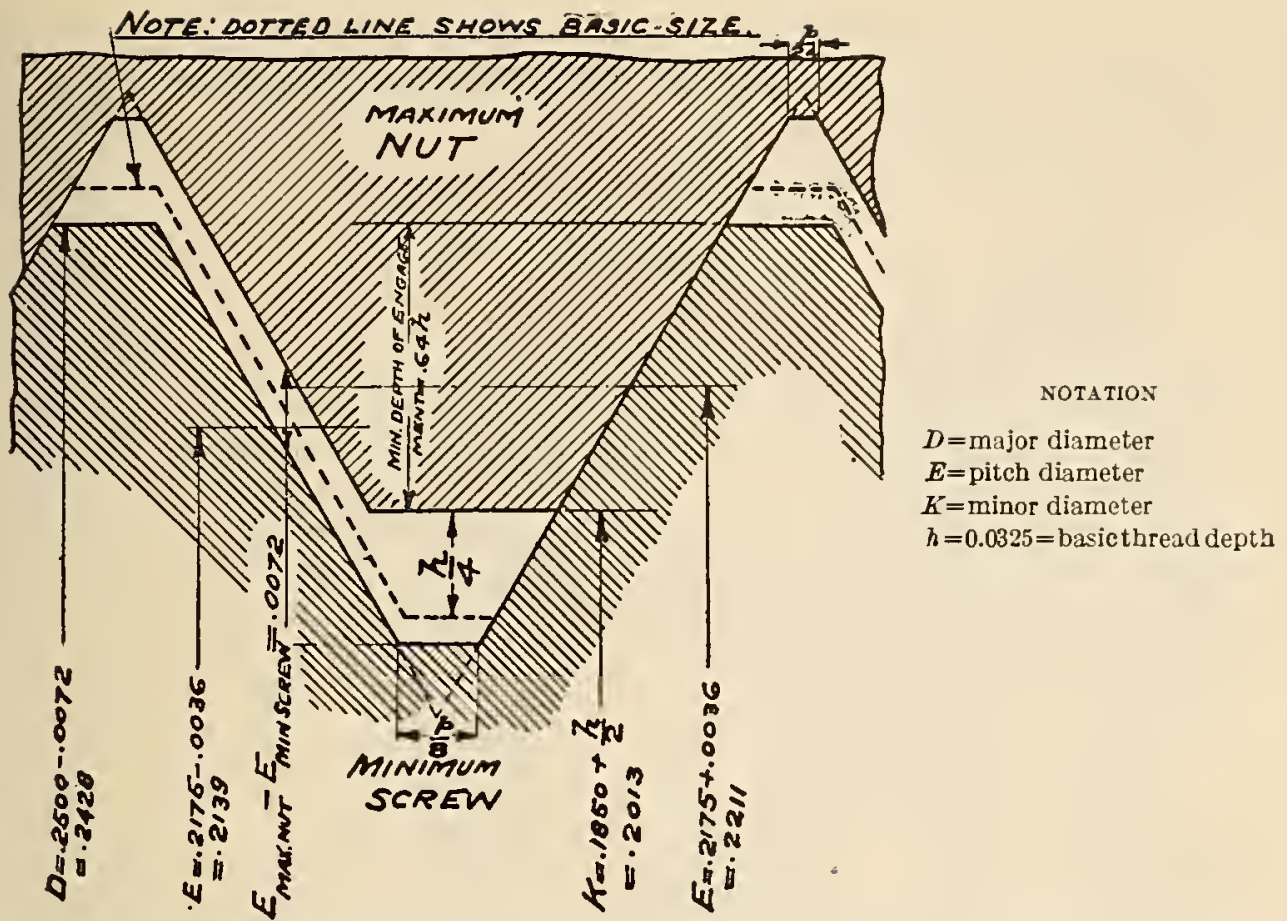

Fig. 8.-Illustration of loosest condition for class 2 , free fit, one-fourth inch, 20 threads

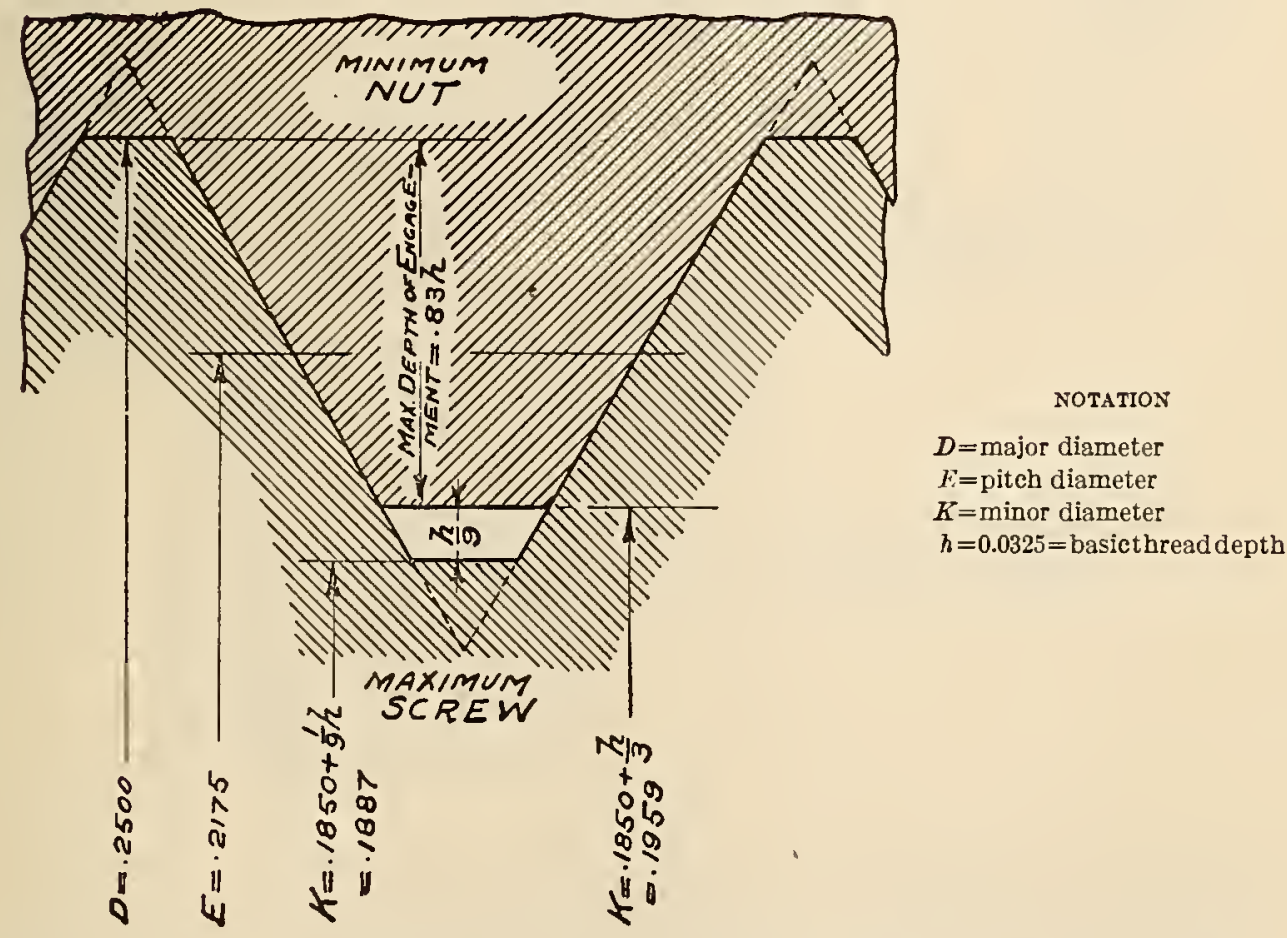

Fig. 9.-Illustration of tightest condition for class 2, free fit, one-fourth inch, 20 $16802^{\circ}-25 \div-3$ threads 
3. Class 3, Medium Fit.-(a) Definition.-The medium-fit class is intended to apply to the manufacture of the higher grade of threaded parts which are to assemble nearly or entirely with the fingers and must have the minimum amount of shake or play between

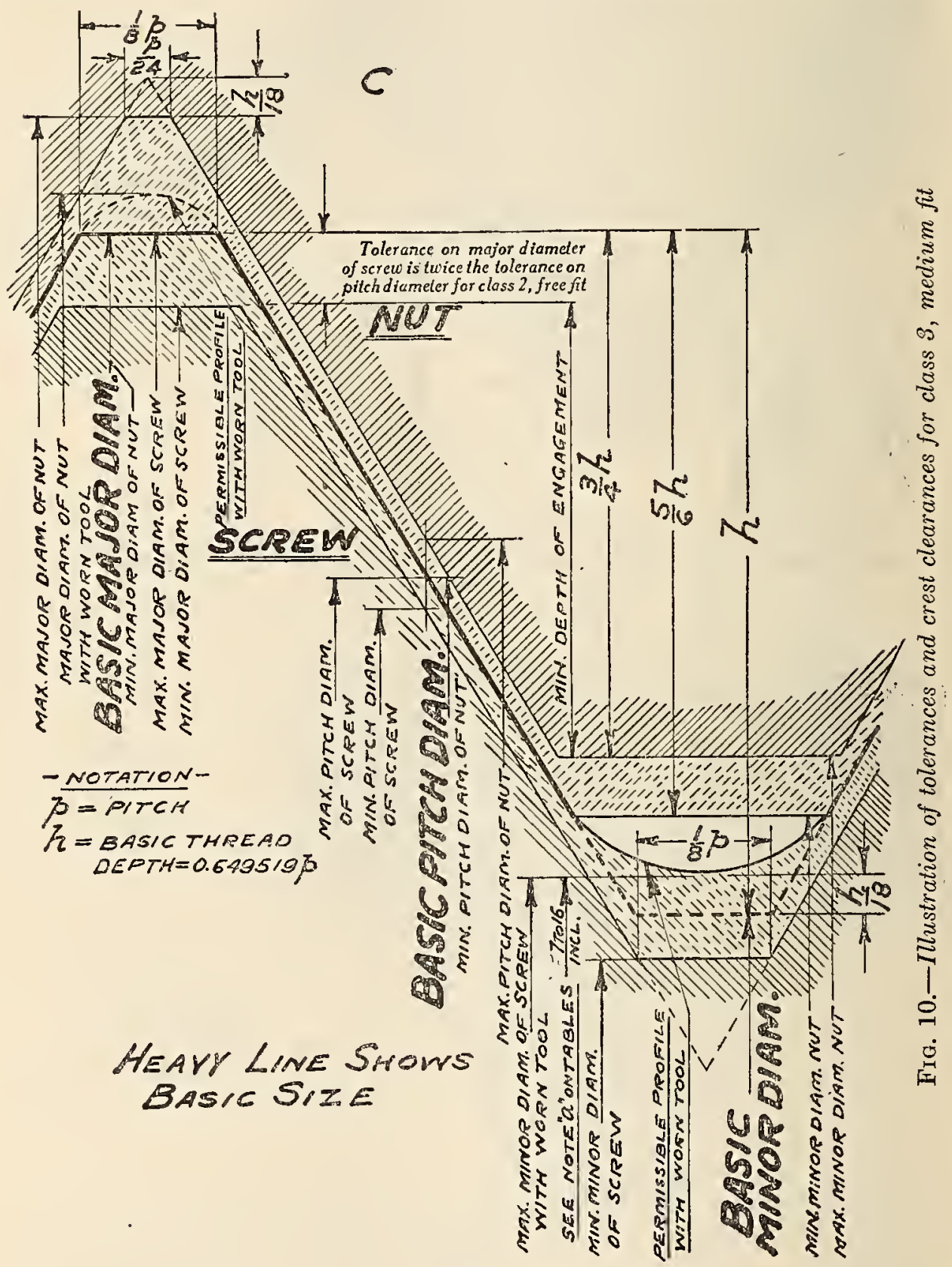

the threaded members. It is the same in every particular as class 2 , free fit, except that the tolerances are smaller.

(b) Minimum nut basic.- The pitch diameter of the minimum nut of a given diameter and pitch corresponds to the basic pitch diameter, as specified in tables of thread series given herein, which is computed from the basic major diameter of the thread. 


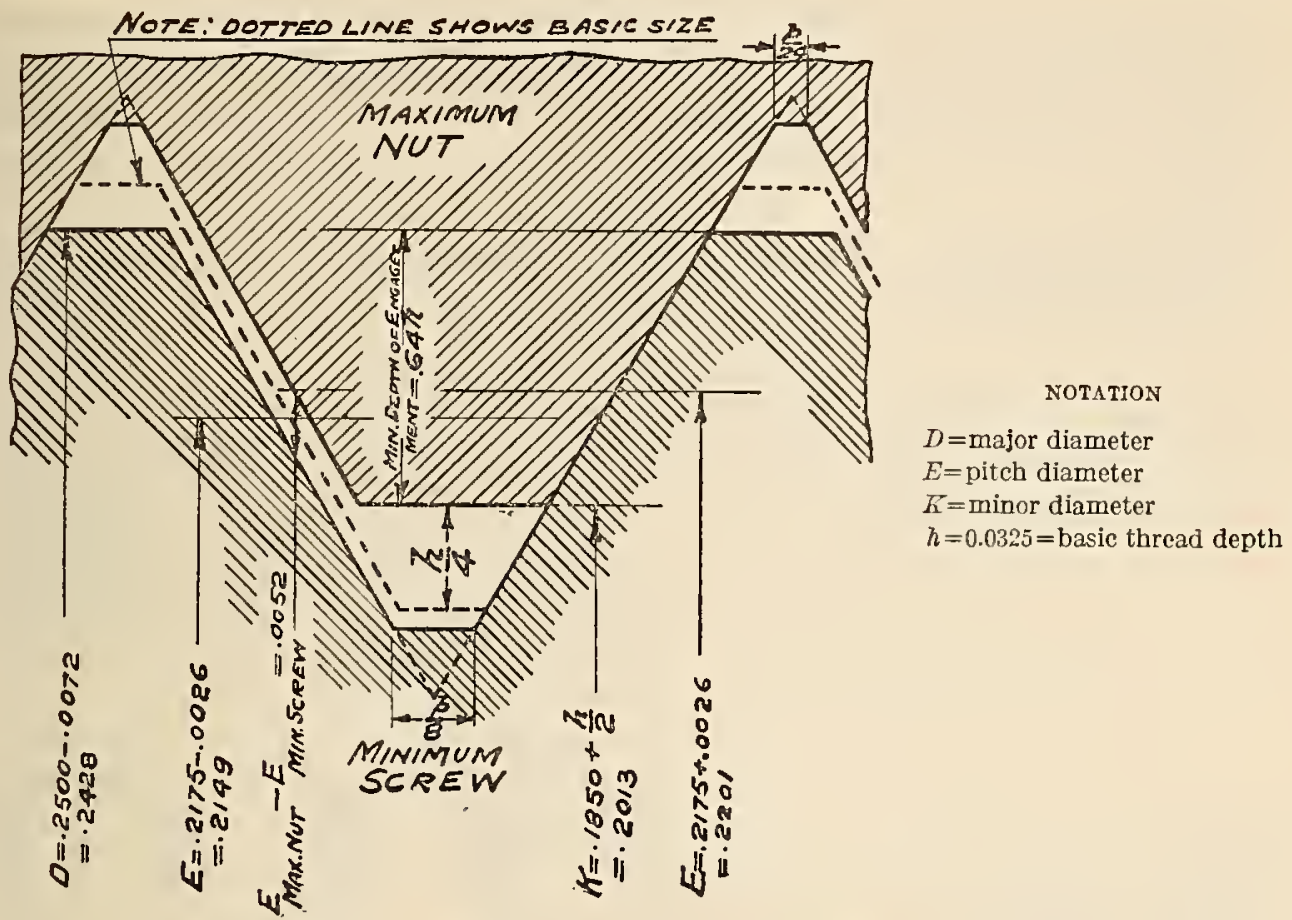

FIG. 11.-1llustration of loosest condition for class 3, medium fit, one-fourth inch, 20 threads

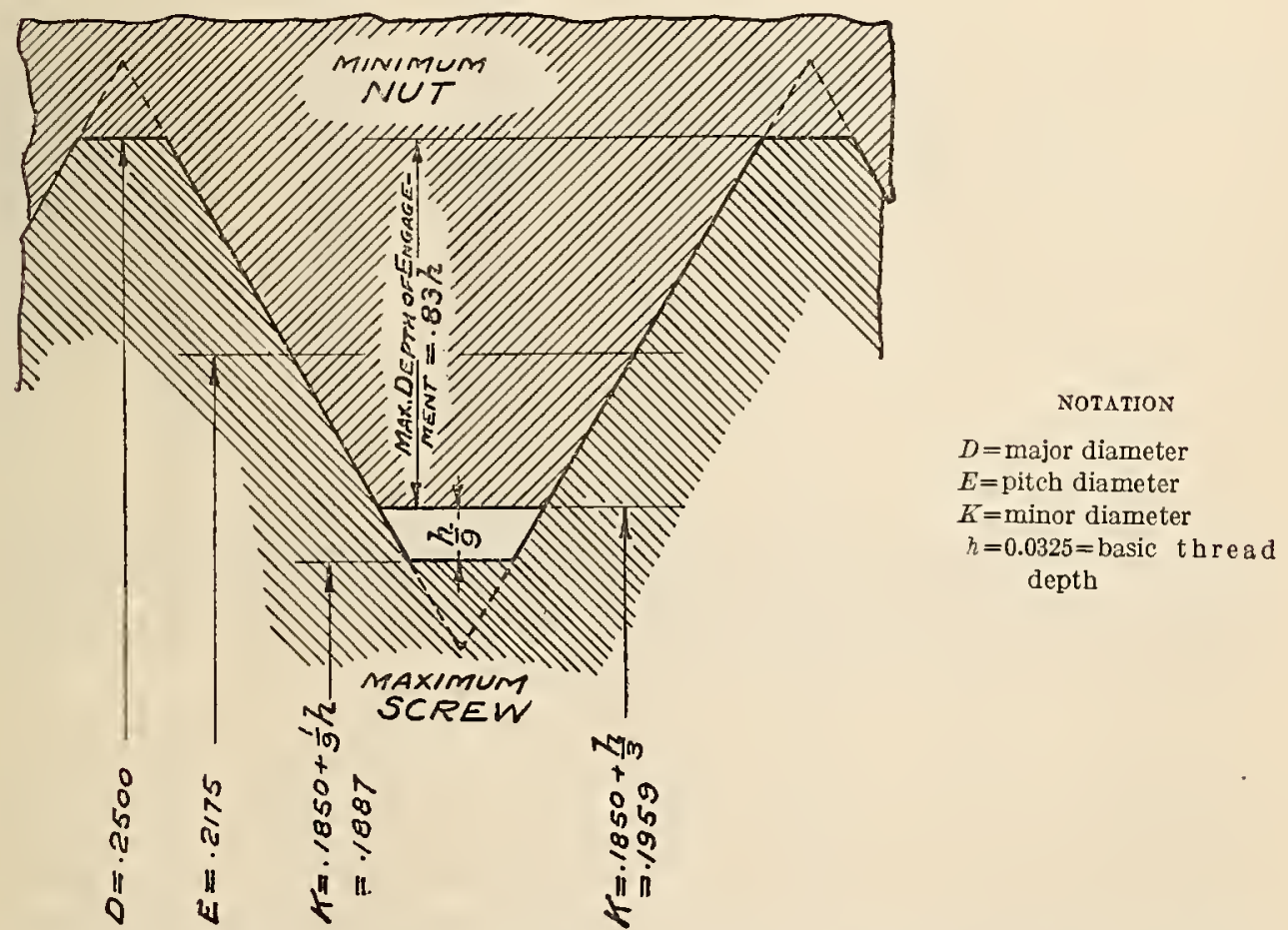

FIG. 12.-Illustration of tightest condition for class 3 , medium fit, one-fourth inch, 20 threads 
(c) Maximum screw basic. ${ }^{5}$ - The major diameter and pitch diameter of the maximum screw of a given pitch and diameter correspond to the basic dimensions, as specified in tables of thread series given herein, which are computed from the basic major diameter of the thread.

(d) Allowance and tolerance values.-Allowances and tolerances are srecified in Table 5.

TABLE 5.-Class 3, medium fit, allowances and tolerances for screws and nuts

\begin{tabular}{|c|c|c|c|c|}
\hline Threads per inch & Allowances & $\begin{array}{c}\text { Pitch- } \\
\text { diameter } \\
\text { tolerancesa }\end{array}$ & $\begin{array}{c}\text { Lead } \\
\text { errors } \\
\text { consuming } \\
\text { one-half } \\
\text { of pitch- } \\
\text { diameter } \\
\text { tolerances s }\end{array}$ & $\begin{array}{l}\text { Errors in } \\
\text { half-angle } \\
\text { consuming } \\
\text { one-half } \\
\text { of pitch- } \\
\text { diameter } \\
\text { tolersnces }\end{array}$ \\
\hline 1 & 2 & 3 & 4 & a \\
\hline $\begin{array}{l}80 \\
72 \\
64 \\
48\end{array}$ & $\begin{array}{c}\text { Inch } \\
0.0000 \\
.0000 \\
.0000 \\
.0000 \\
.0000\end{array}$ & $\begin{array}{l}\text { Inch } \\
0.0013 \\
.0013 \\
.0014 \\
.0015 \\
.0016\end{array}$ & $\begin{array}{c}\text { Inch } \\
0.0004 \\
.0004 \\
.0004 \\
.0004 \\
.0005\end{array}$ & $\begin{array}{rrr}\text { Deg. } & \text { Min. } \\
1 & 59 \\
1 & 47 \\
1 & 43 \\
1 & 35 \\
1 & 28\end{array}$ \\
\hline $\begin{array}{l}44 \\
40 \\
36\end{array}$ & $\begin{array}{l}.0000 \\
.0000 \\
.0000 \\
.0000 \\
.0000\end{array}$ & $\begin{array}{l}.0016 \\
.0017 \\
.0018 \\
.0019 \\
.0022\end{array}$ & $\begin{array}{l}.0005 \\
.0005 \\
.0005 \\
.0005 \\
.0006\end{array}$ & $\begin{array}{ll}1 & 21 \\
1 & 18 \\
1 & 14 \\
1 & 10 \\
1 & 11\end{array}$ \\
\hline 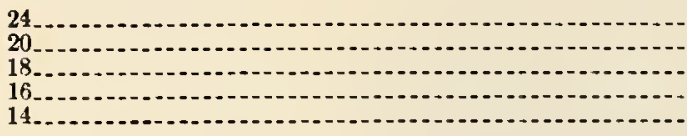 & $\begin{array}{l}.0000 \\
.0000 \\
.0000 \\
.0000 \\
.0000\end{array}$ & $\begin{array}{l}.0024 \\
.0026 \\
.0030 \\
.0032 \\
.0036\end{array}$ & $\begin{array}{l}.0007 \\
.0008 \\
.0009 \\
.0009 \\
.0010\end{array}$ & $\begin{array}{lr}1 & 6 \\
1 & 0 \\
1 & 2 \\
0 & 59 \\
0 & 58\end{array}$ \\
\hline $11_{10}^{13}$ & $\begin{array}{l}.0000 \\
.0000 \\
.0000 \\
.0000 \\
.0000\end{array}$ & $\begin{array}{l}.0037 \\
.0040 \\
.0042 \\
.0045 \\
.0049\end{array}$ & $\begin{array}{l}.0011 \\
.0012 \\
.0012 \\
.0013 \\
.0014\end{array}$ & $\begin{array}{l}0 \\
0 \\
0 \\
0 \\
0\end{array}$ \\
\hline $\begin{array}{l}8 \\
6 \\
6 \\
5 \\
4\end{array}$ & $\begin{array}{l}.0000 \\
.0000 \\
.0000 \\
.0000 \\
.0000\end{array}$ & $\begin{array}{l}.0054 \\
.0059 \\
.0071 \\
.0082 \\
.0089\end{array}$ & $\begin{array}{l}.0016 \\
.0017 \\
.0020 \\
.0024 \\
.0026\end{array}$ & $\begin{array}{l}0 \\
0 \\
0 \\
0 \\
0\end{array}$ \\
\hline $4 \ldots$ & .0000 & .0097 & .0028 & 0 \\
\hline
\end{tabular}

a The tolerances spccified for pitch diameter include all crrors of pitch diameter, lead, and angle. The full tolerance can not, therefore, be used on pitch diameter unless the lead and angle of the thread are perfect. Columns 4 and 5 give, for information, the errors in lead (per length of thread engaged) and in angle, each of which can be compensated for hy half the tolerance on the pitch diameter given in column 3 . If lead and angle errors hoth exist to the amount tabulated, the pitch diameter of a holt, for example, must be reduced angle errors hoth exist to the amount tabulated, the pitch
by the full tolerance or it will not enter a basic nut or gage.

$o$ Between any two throads not farther apart than the length of engagement.

4. Class 4, Close Fit.-(a) Definition.-The close-fit class is intended for threaded work of the finest commercial quality where very little shake or play is desirable, and where a screw driver or wrench may be necessary for assembly. In the manufacture of screw-thread products belonging in this class it will be necessary to use precision tools, selected gages, and other refinements. This quality of work should, therefore, be used only in cases where requirements of the

\footnotetext{
- The maximum minor diameter of the screw is ahove the hasic minor diamoter, as shown in Figurc 10.
} 
mechanism being produced are exacting, or where special conditions requirescrews having a precision fit. In order to secure the fit desired it may be necessary in some cases to select the parts when the product is being assembled.

(b) Minimum nut basic. - The pitch diameter of the minimum nut of a given diameter and pitch corresponds to the basic pitch diameter, as specified in tables of thread series given herein, which is computed from the basic major diameter of the thread.

(c) Maximum screw above basic. - The pitch diameter of the maximum screw of a given diameter and pitch is above the basic dimensions, as specified in tables of thread series given herein, which are computed from the basic major diameter of the thread, by the amount of the allowance (interference) specified in Table 6.

(d) Allowance and tolerance values. - Allowances and tolerances are specified in Table 6 .

TABLE 6.-Class 4, close fit, allowances and tolerances for screws and nuts

\begin{tabular}{|c|c|c|c|c|c|}
\hline Threads per inch & $\begin{array}{c}\text { 1nterfer- } \\
\text { ences or } \\
\text { negative } \\
\text { allowances }\end{array}$ & $\begin{array}{c}\text { Pitch- } \\
\text { diameter } \\
\text { tolerances } 1\end{array}$ & $\begin{array}{c}\text { Lead } \\
\text { errors } \\
\text { consuming } \\
\text { one-half of } \\
\text { pitch- } \\
\text { diameter } \\
\text { tolerances? }\end{array}$ & \multicolumn{2}{|c|}{$\begin{array}{l}\text { Errors in } \\
\text { balf-angle } \\
\text { consuming } \\
\text { one-half of } \\
\text { pitch- } \\
\text { diameter } \\
\text { tolcrances }\end{array}$} \\
\hline 1 & 2 & 3 & 4 & 5 & \\
\hline $\begin{array}{l}80 \\
764 \\
56\end{array}$ & $\begin{array}{c}\text { Inch } \\
0.0001 \\
.0001 \\
.0001 \\
.0002 \\
.0002\end{array}$ & $\begin{array}{l}\text { Inch } \\
0.0006 \\
.0007 \\
.0007 \\
.0007 \\
.0008\end{array}$ & $\begin{array}{c}\text { Inch } \\
0.0002 \\
.0002 \\
.0002 \\
.0002 \\
.0002\end{array}$ & $\begin{array}{c}\text { Deg. } \\
0 \\
0 \\
0 \\
0 \\
0\end{array}$ & $\begin{array}{r}\text { Min. } \\
55 \\
58 \\
51 \\
45 \\
44\end{array}$ \\
\hline $\begin{array}{l}44-20 \\
40 \\
36 \\
32 \\
28\end{array}$ & $\begin{array}{l}.0002 \\
.0002 \\
.0002 \\
.0002 \\
.0002\end{array}$ & $\begin{array}{l}.0008 \\
.0009 \\
.0009 \\
.0010 \\
.0011\end{array}$ & $\begin{array}{l}.0002 \\
.0003 \\
.0003 \\
.0003 \\
.0003\end{array}$ & $\begin{array}{l}0 \\
0 \\
0 \\
0 \\
0\end{array}$ & $\begin{array}{l}40 \\
41 \\
37 \\
37 \\
35\end{array}$ \\
\hline 1840. & $\begin{array}{l}.0003 \\
.0003 \\
.0003 \\
.0004 \\
.0004\end{array}$ & $\begin{array}{l}.0012 \\
.0013 \\
.0015 \\
.0016 \\
.0018\end{array}$ & $\begin{array}{l}.0003 \\
.0004 \\
.0004 \\
.0005 \\
.0005\end{array}$ & $\begin{array}{l}0 \\
0 \\
0 \\
0 \\
0\end{array}$ & $\begin{array}{l}33 \\
30 \\
31 \\
29 \\
29\end{array}$ \\
\hline 1312 & $\begin{array}{l}.0004 \\
.0005 \\
.0005 \\
.0006 \\
.0006\end{array}$ & $\begin{array}{l}.0019 \\
.0020 \\
.0021 \\
.0023 \\
.0024\end{array}$ & $\begin{array}{l}.0005 \\
.0006 \\
.0006 \\
.0007 \\
.0007\end{array}$ & $\begin{array}{l}0 \\
0 \\
0 \\
0 \\
0\end{array}$ & $\begin{array}{l}28 \\
28 \\
26 \\
26 \\
25\end{array}$ \\
\hline $\begin{array}{l}8 \\
6 \\
4\end{array}$ & $\begin{array}{l}.0007 \\
.0008 \\
.0009 \\
.0010 \\
.0011\end{array}$ & $\begin{array}{l}.0027 \\
.0030 \\
.0036 \\
.0041 \\
.0044\end{array}$ & $\begin{array}{l}.0008 \\
.0009 \\
.0010 \\
.0012 \\
.0013\end{array}$ & $\begin{array}{l}0 \\
0 \\
0 \\
0 \\
0\end{array}$ & $\begin{array}{l}25 \\
24 \\
25 \\
23 \\
23\end{array}$ \\
\hline & .0013 & .0048 & .0014 & 0 & 22 \\
\hline
\end{tabular}

1 The tolerances specified for pitch diameter include all errorsof pitch diameter, lead, and angle. The full tolerance can not, therefore, be used on pitch diameter unless the lead and angle of the thread are perfect. Columns 4 and 5 give, for information, the errors in lead (per length of thread engaged) and in angle, each of which can be compensated for by half the tolerance on the pitch diameter given in column 3 . If lead and angle errors both exist to the amount tabulated, the pitch diameter of a bolt, for example, must be reduced by the full tolerance or it will not enter a basic nut or gage.

2 Between any two threads not farther apart than the length of engagement. 


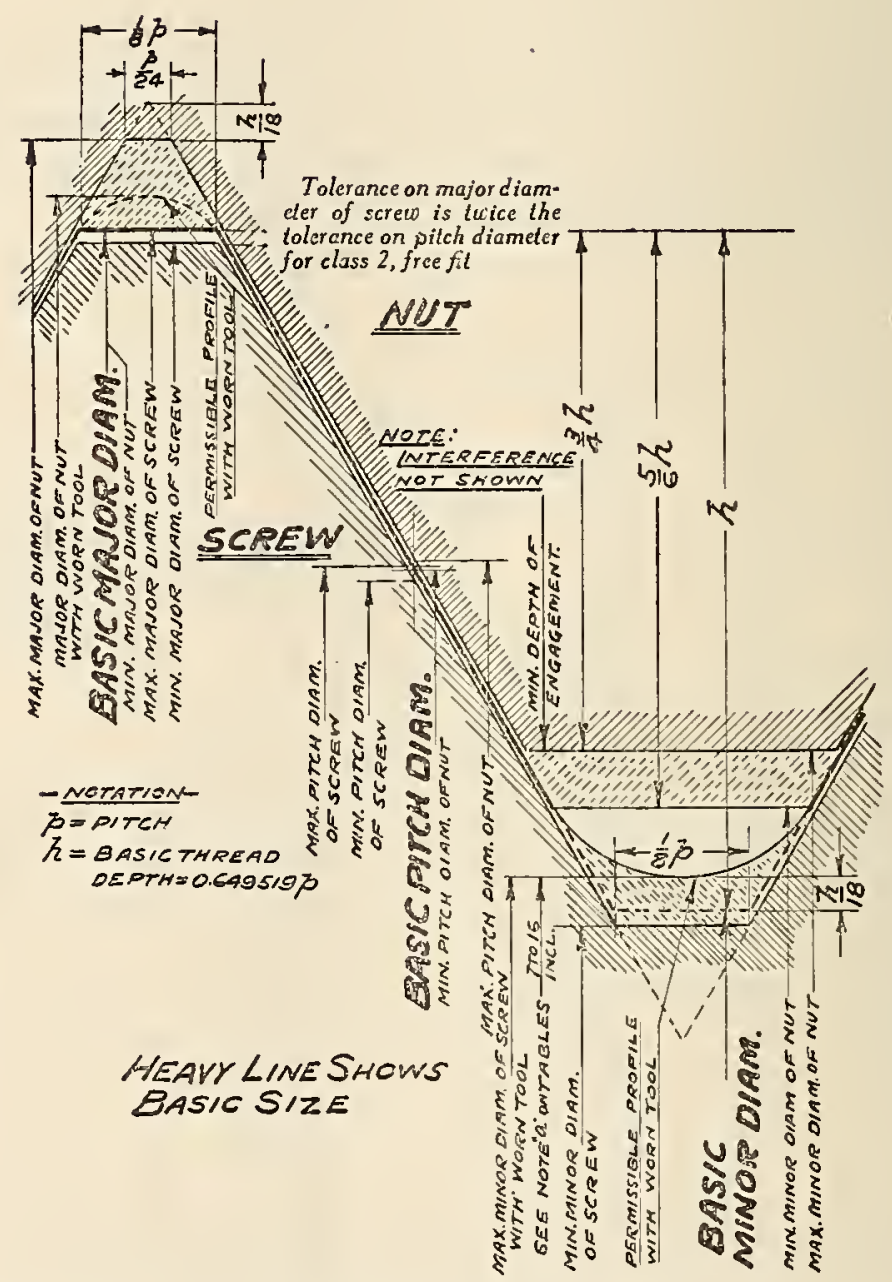

FIG. 13.-Illustration of tolerances, allowance (interference), and crest clearances for class 4, close fit 


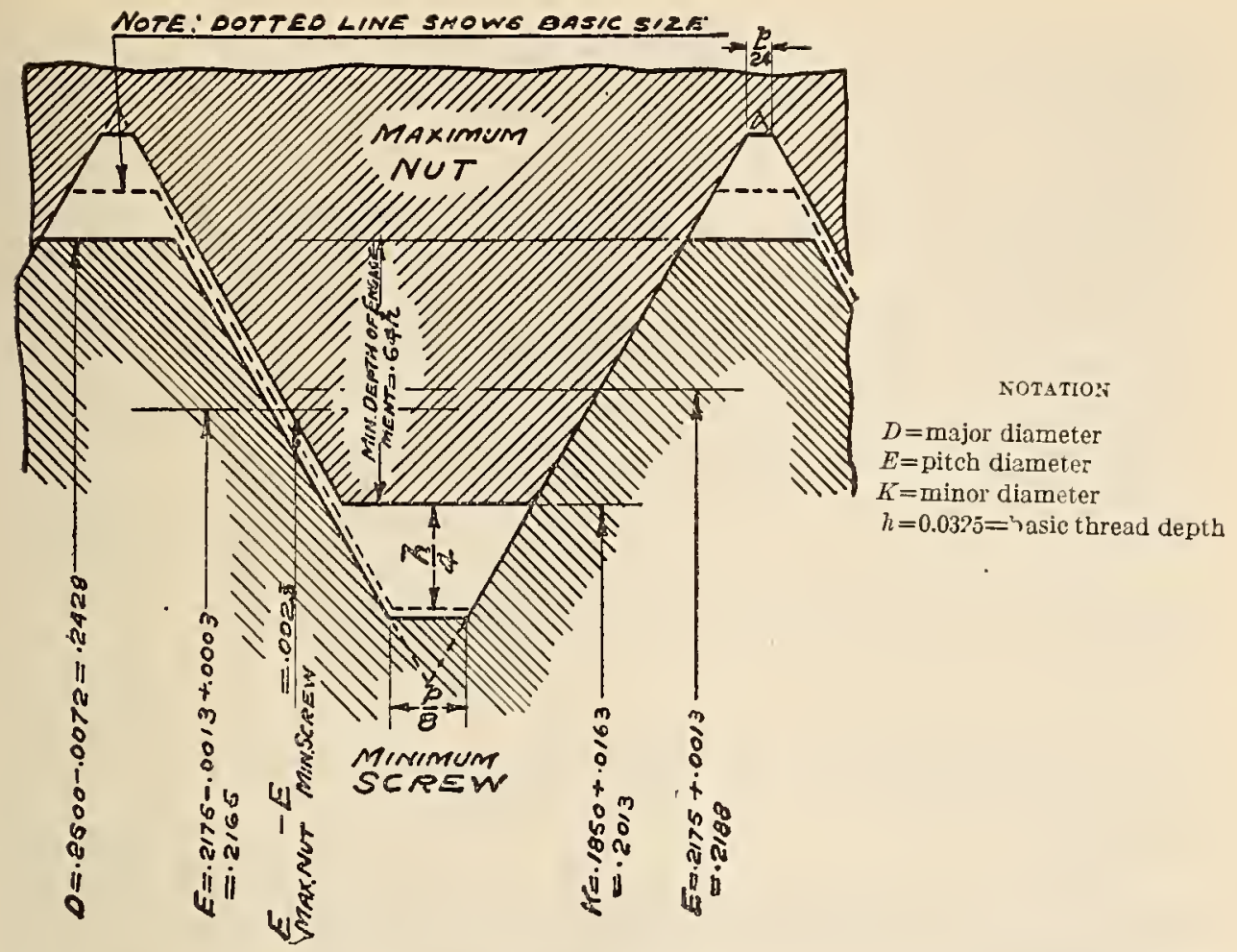
Fig. 14.- Illustration of loosest condition for class 4, close fit, one-fourth inch,
20 threads

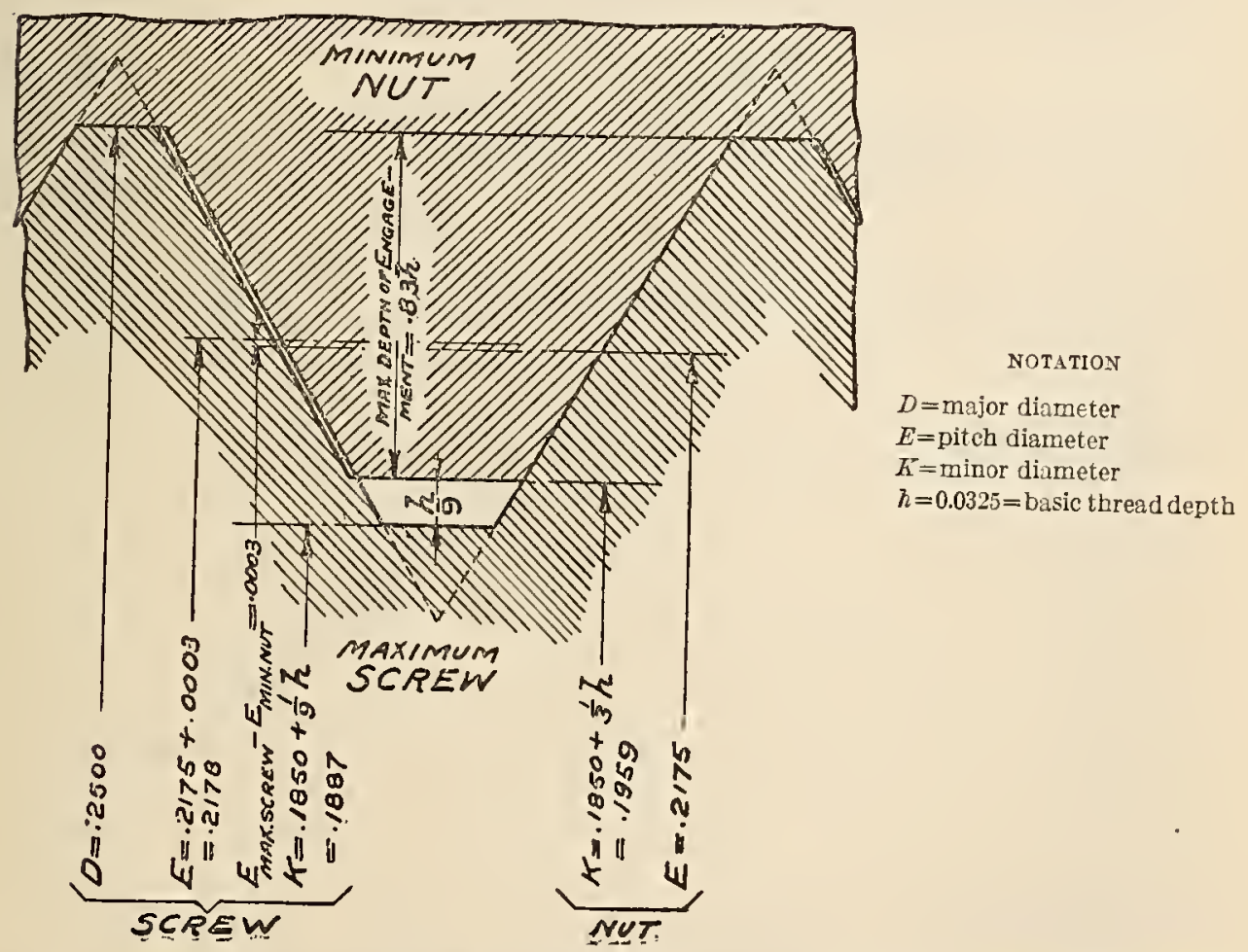

FIG. 15.-Illustration of tightest condition for class 4, close fit, one-fourth inch, 20 threads 


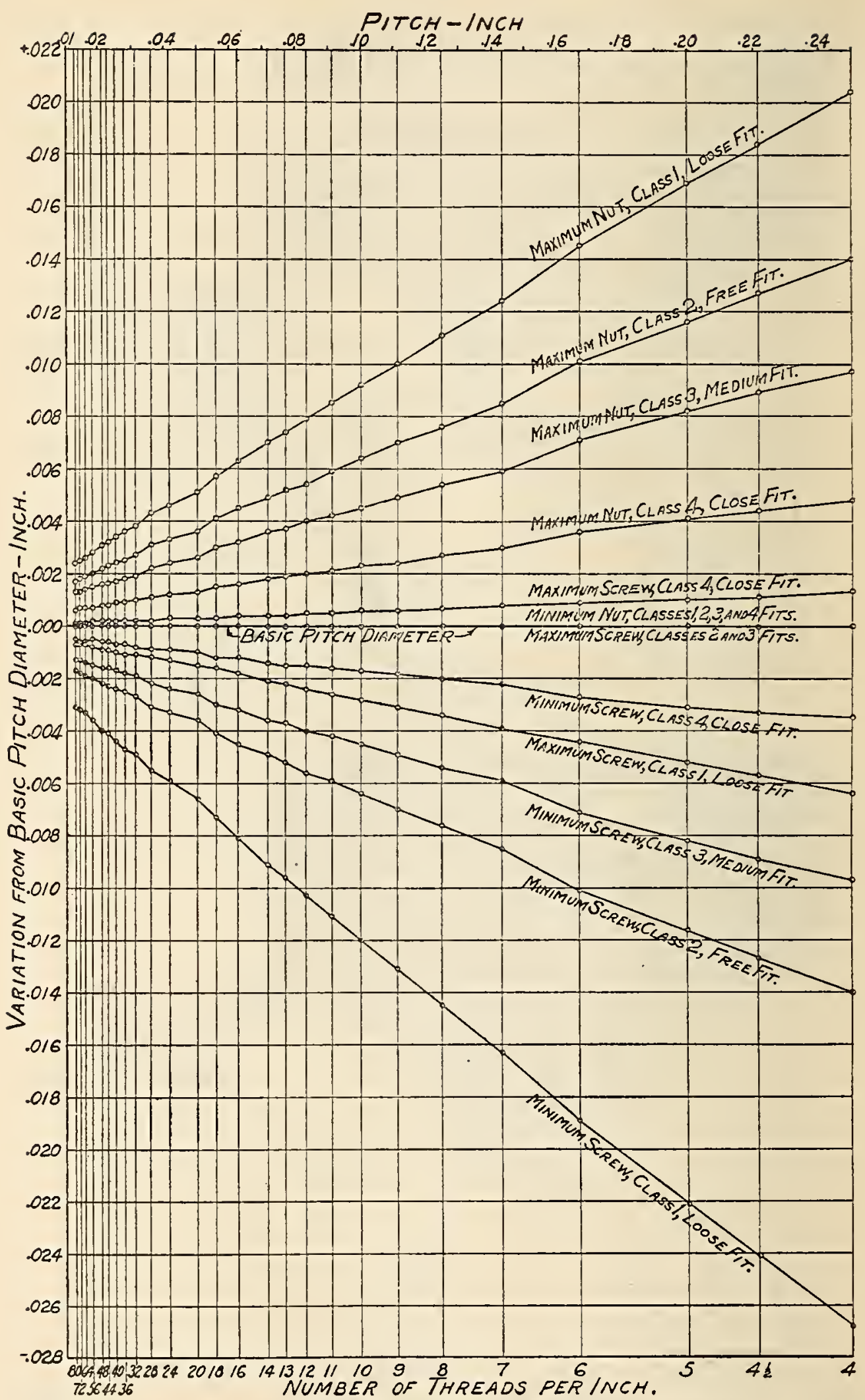

FIG. 16.-Relation of maximum and minimum pitch diameters of classes $1,2,3$, and 4 fits to basic pitch diameters 


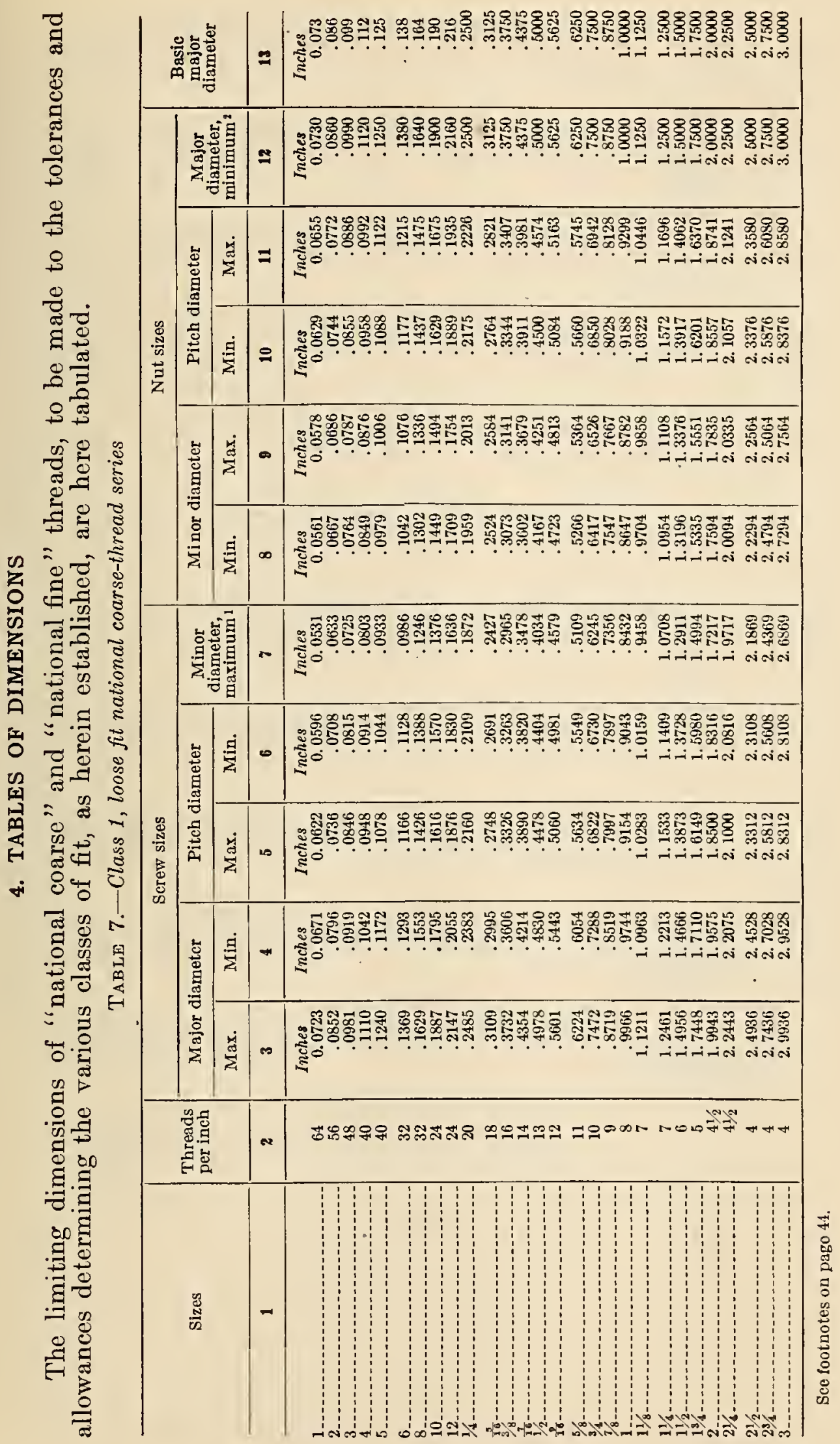




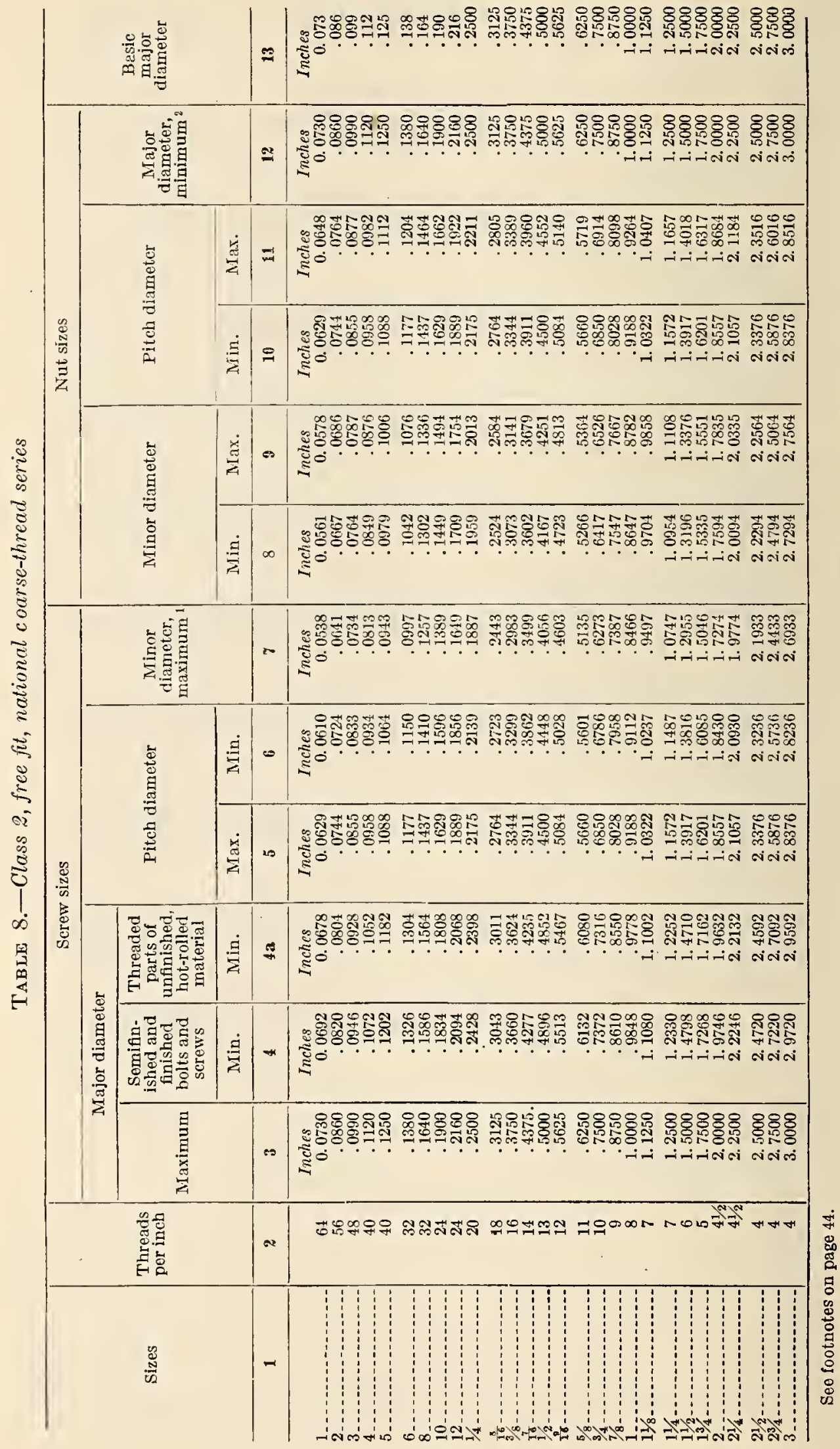




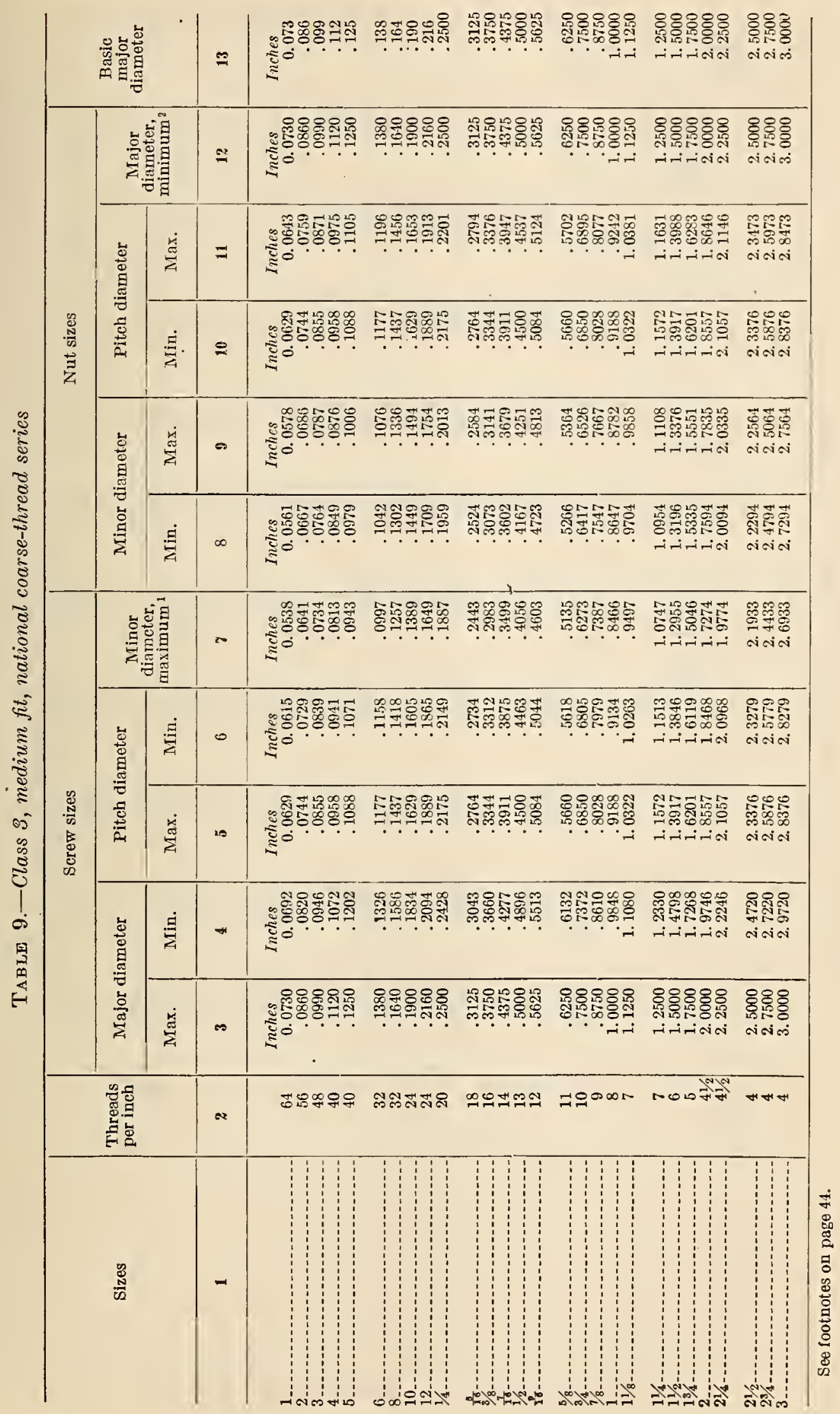




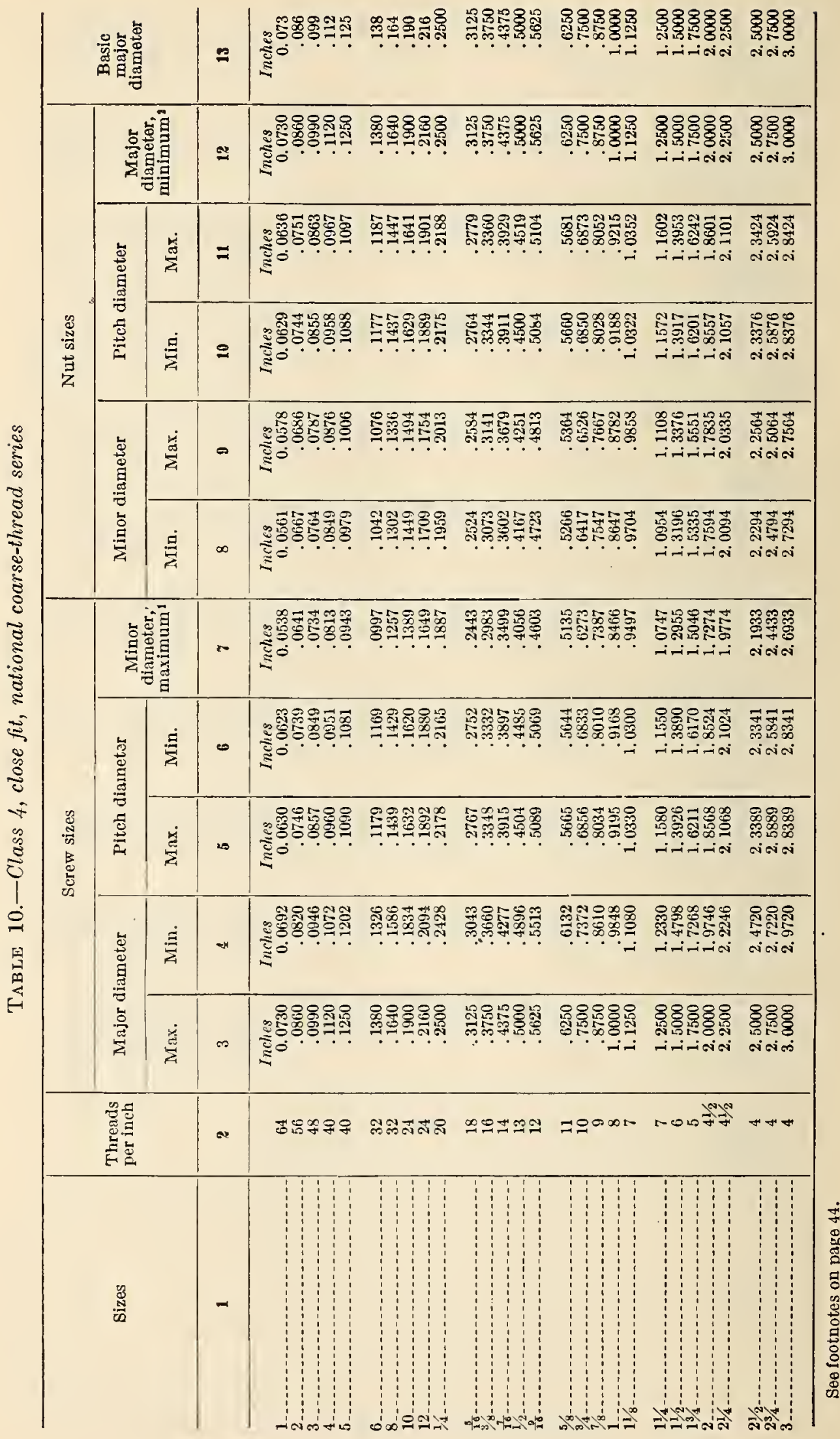


1924 PEPORT

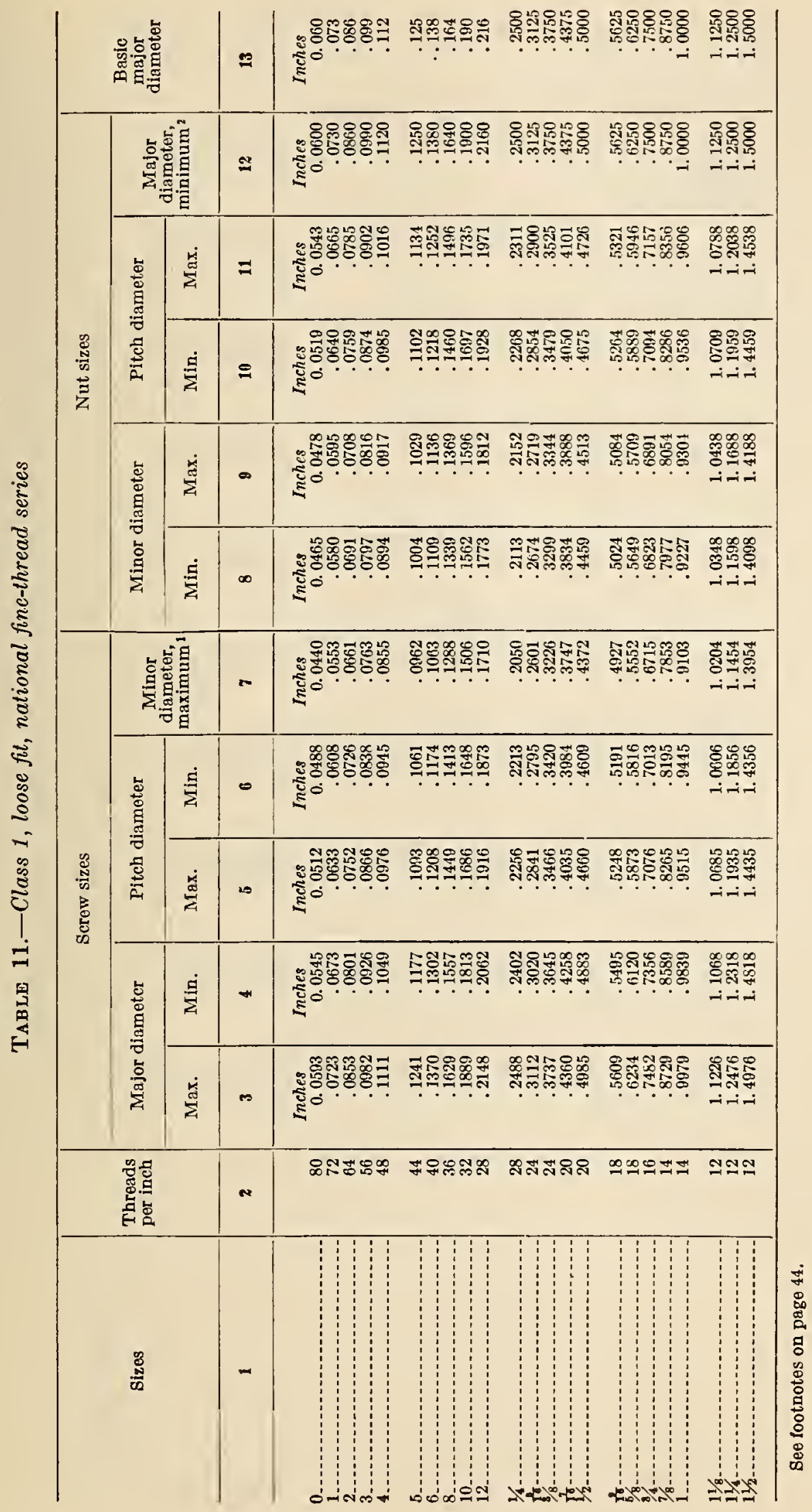




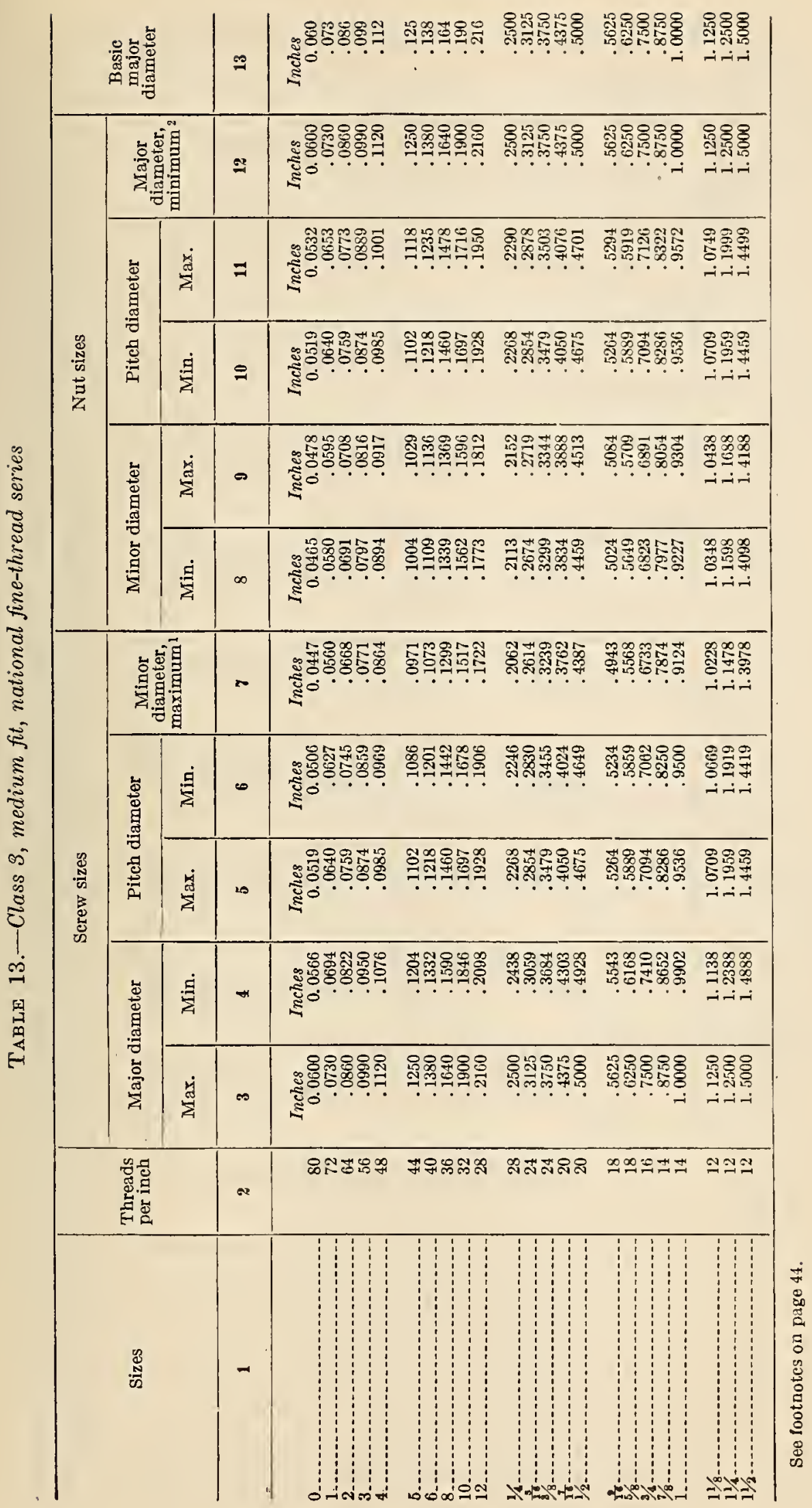




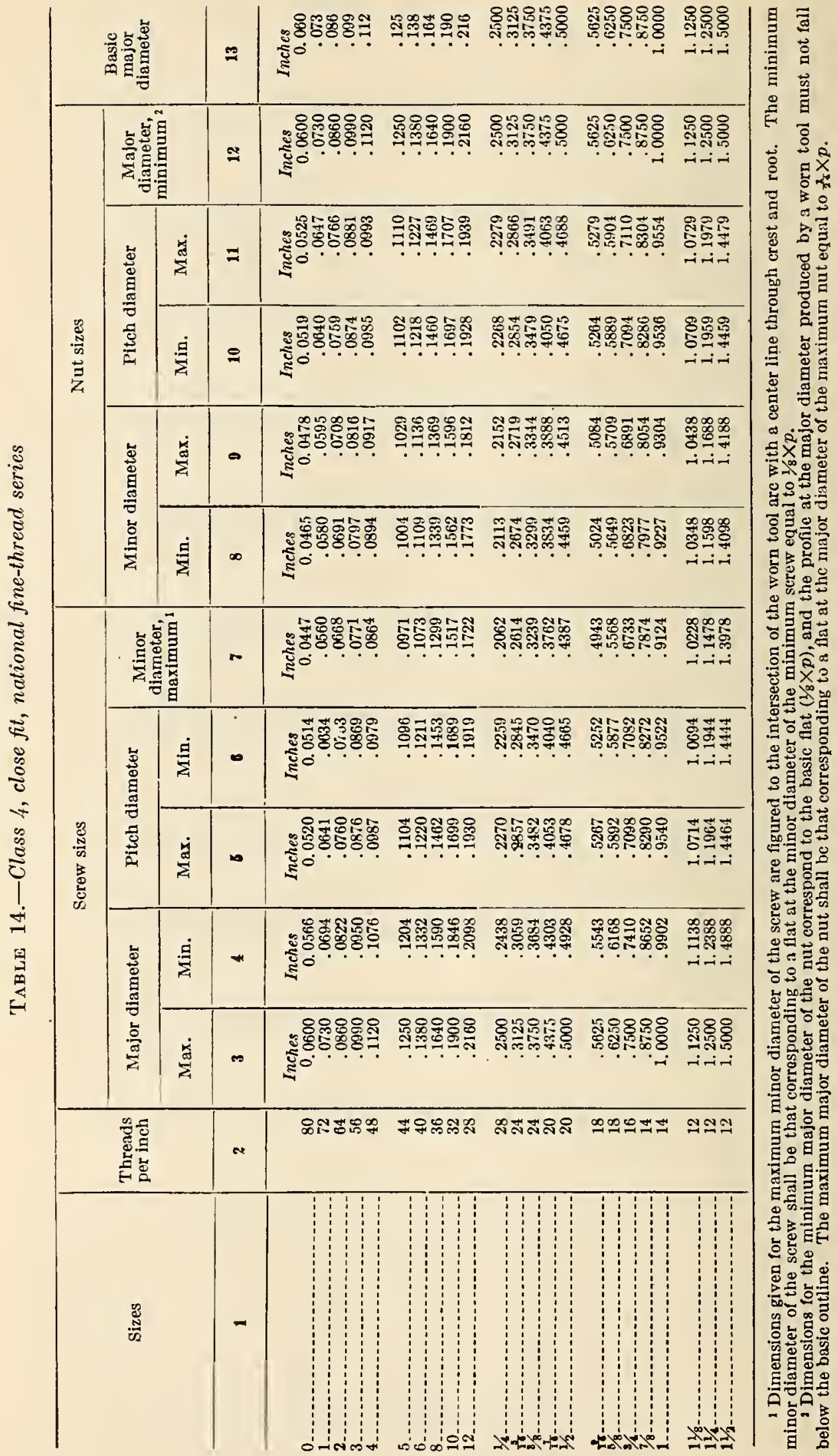




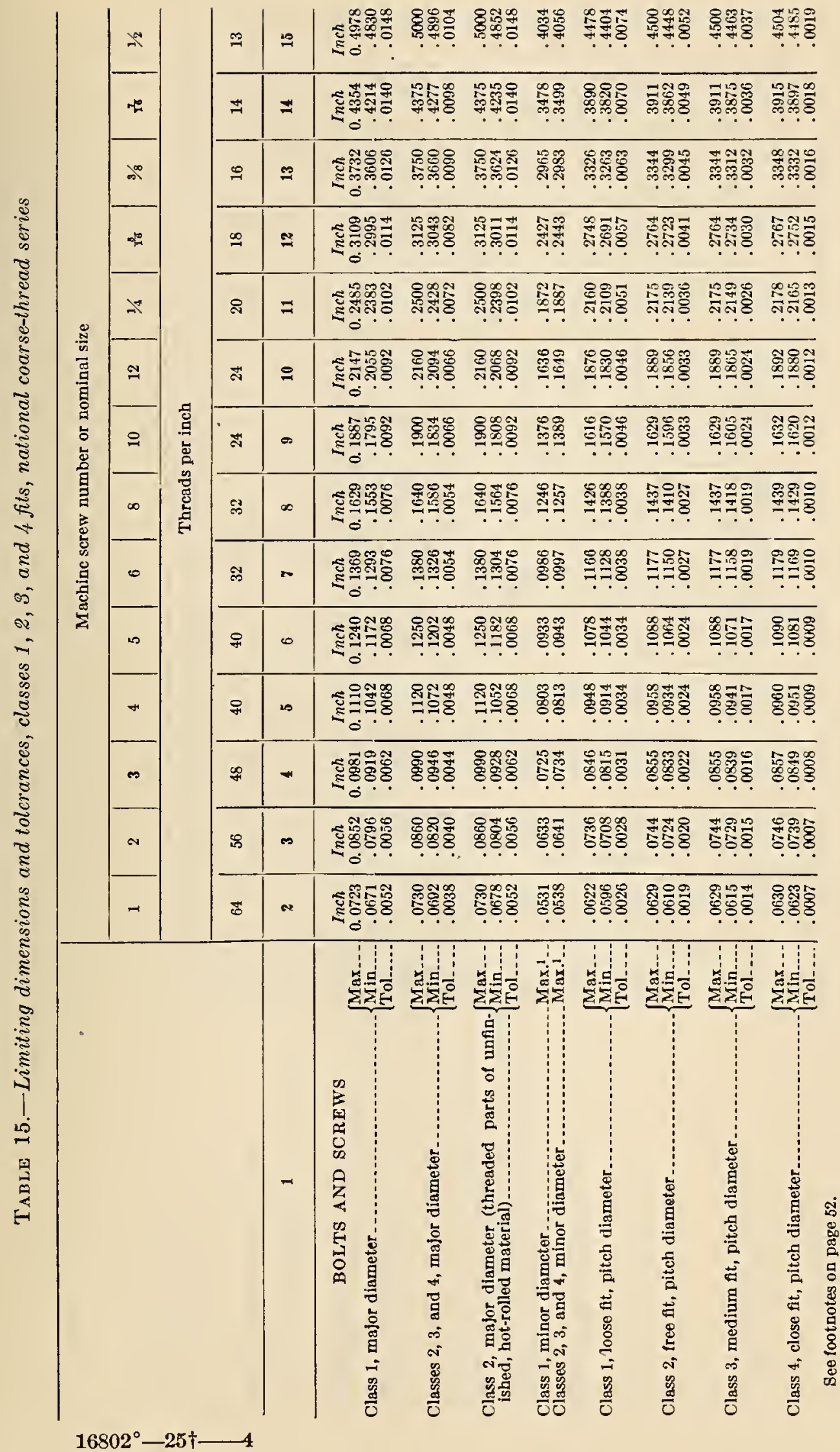




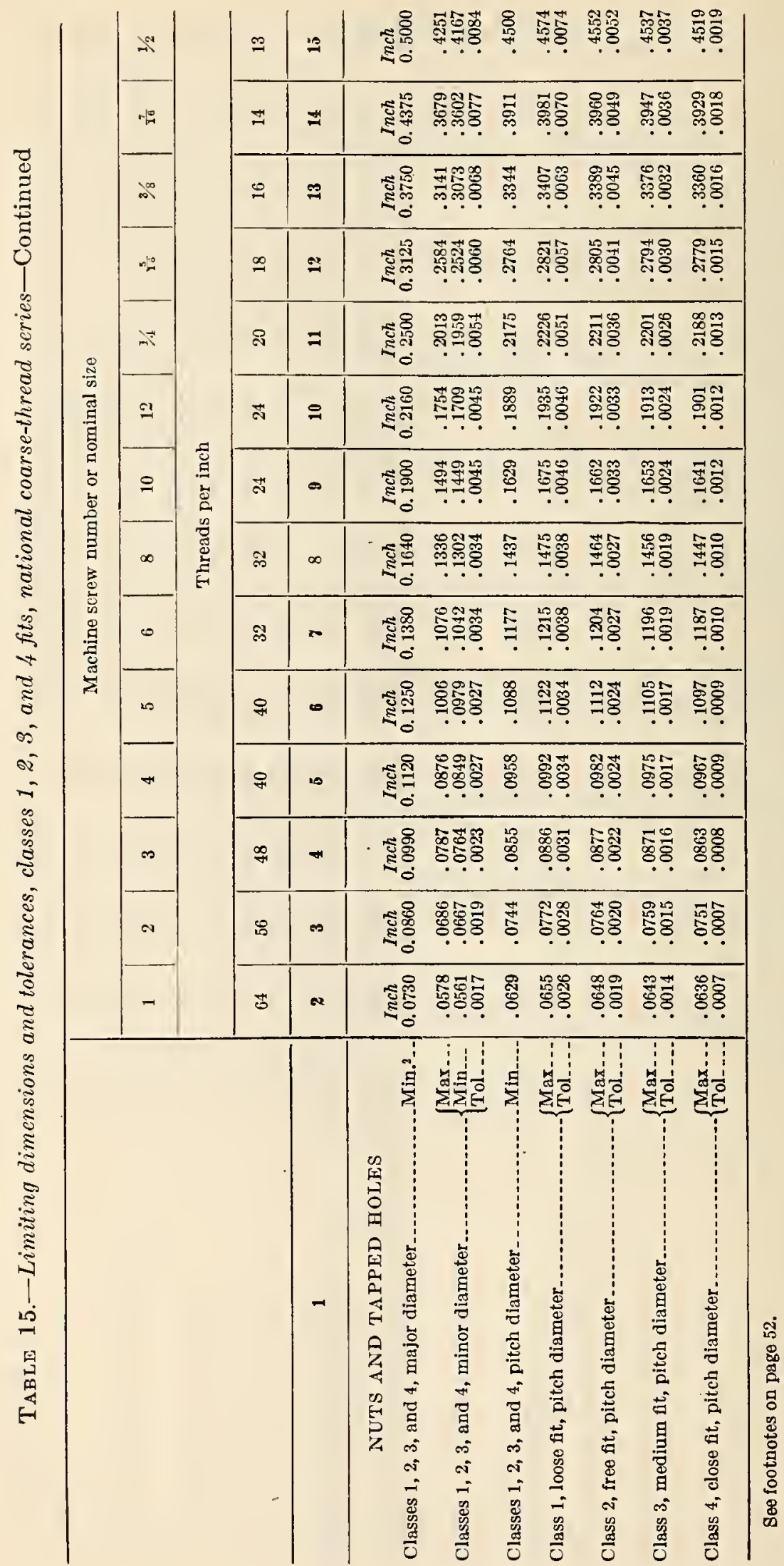




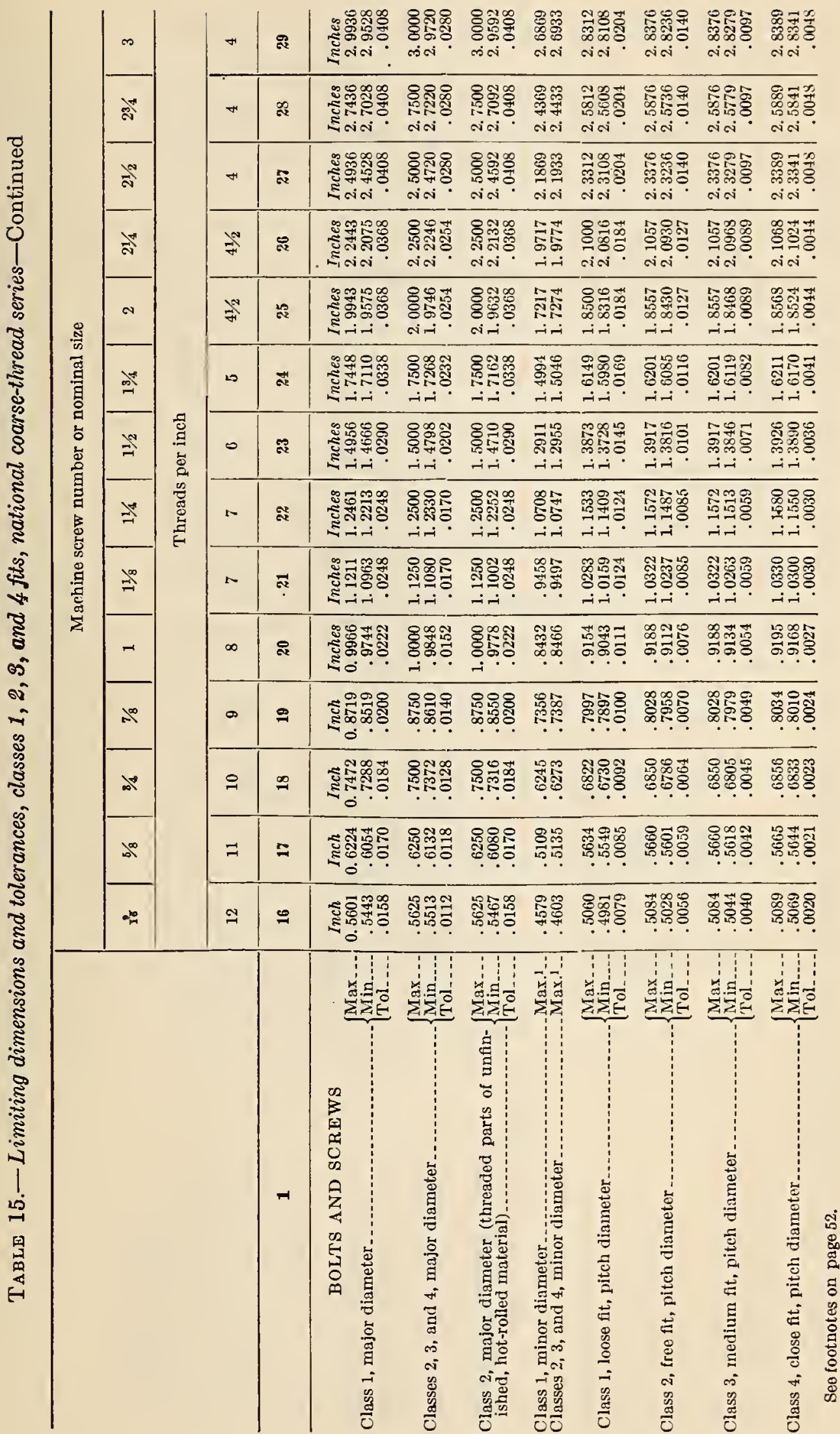




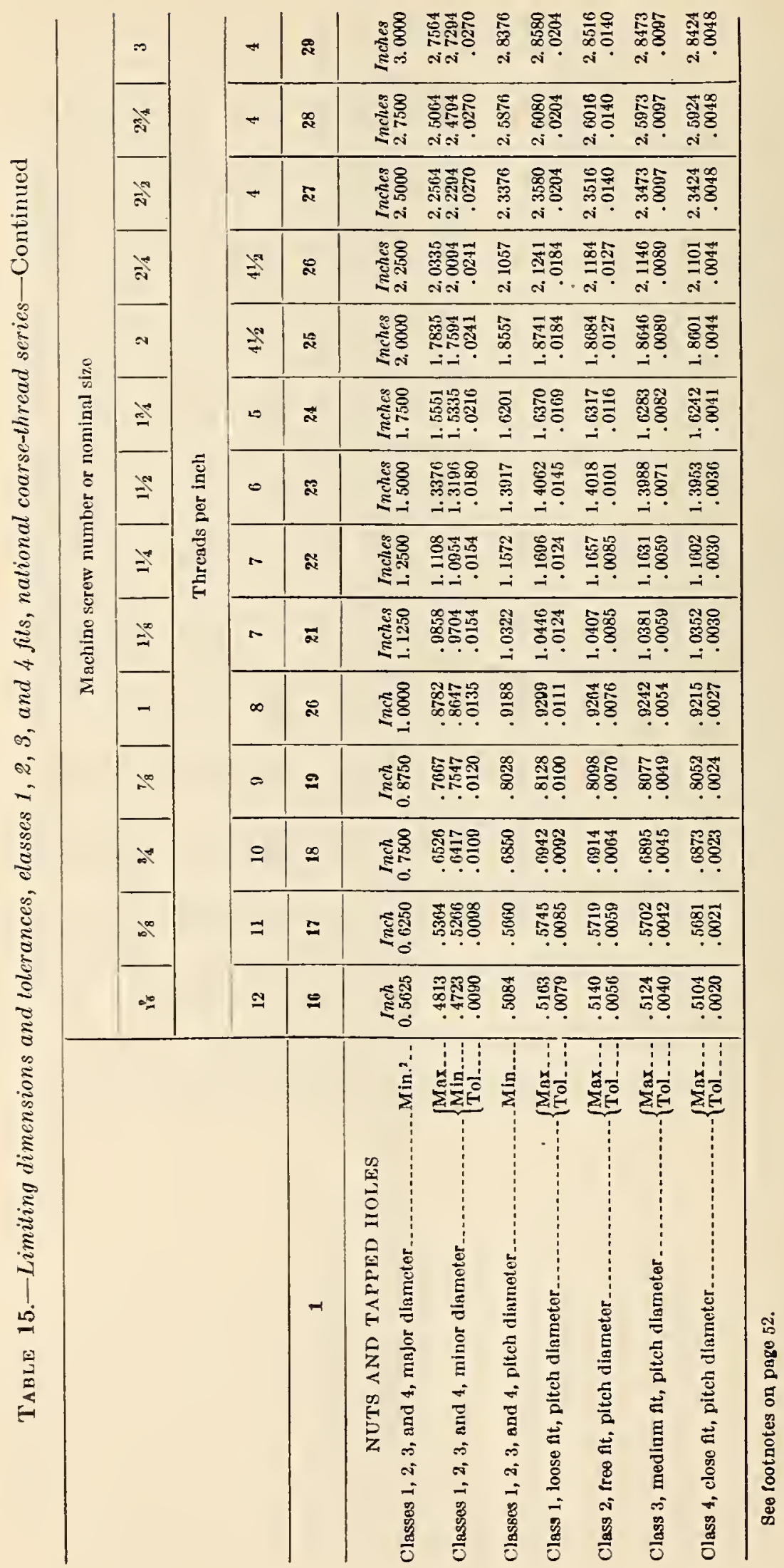




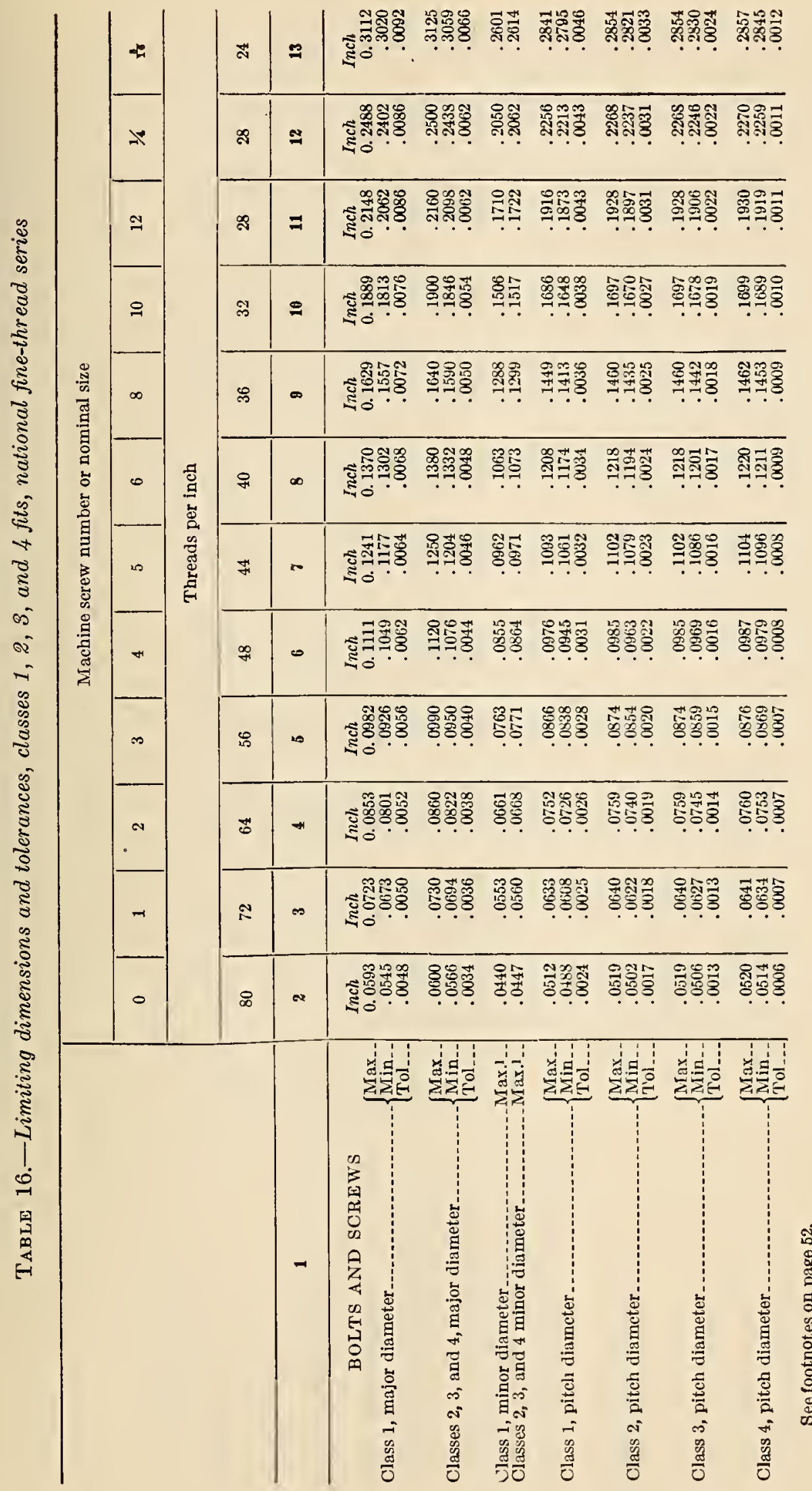




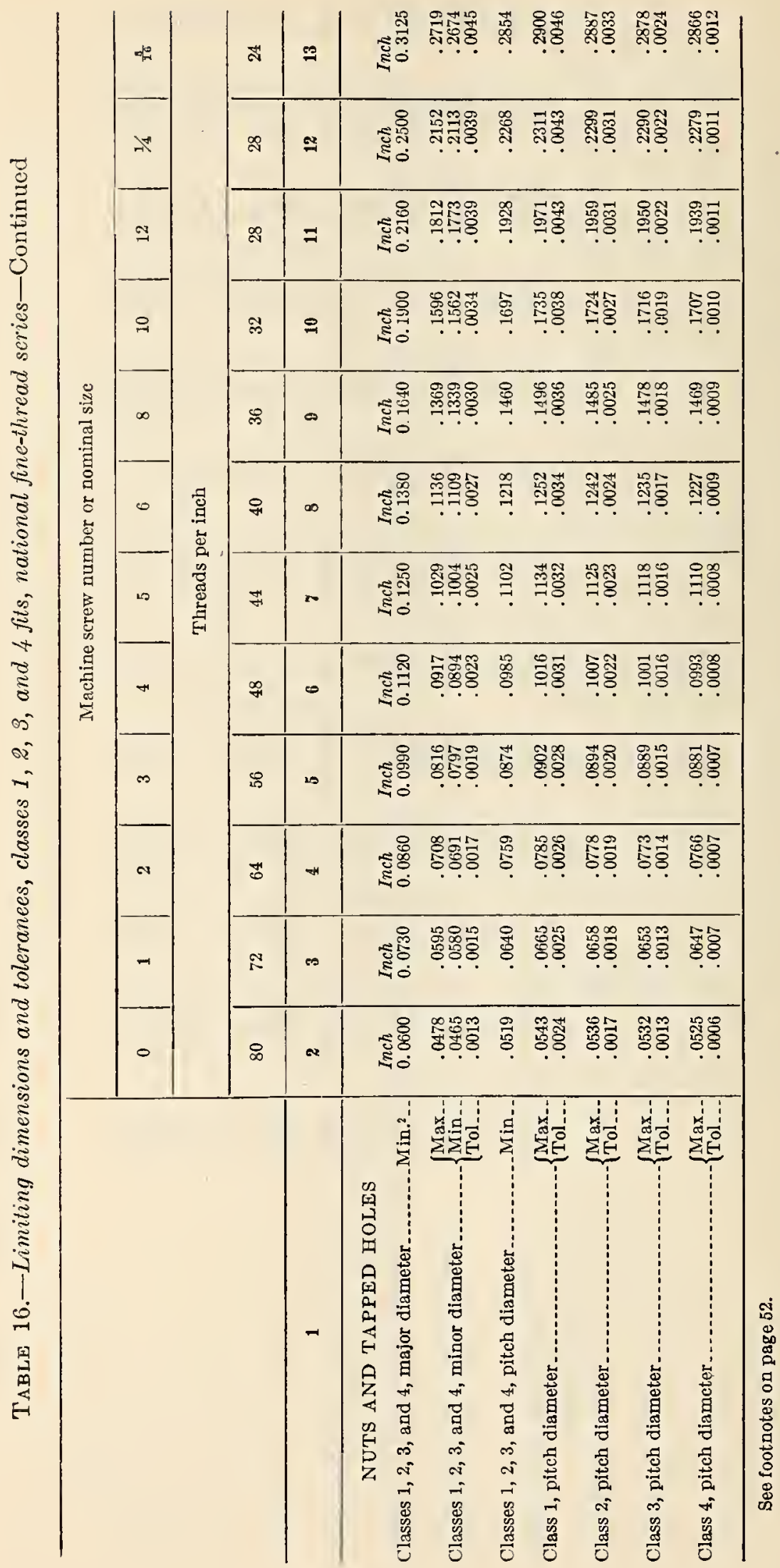


1924 REPORT

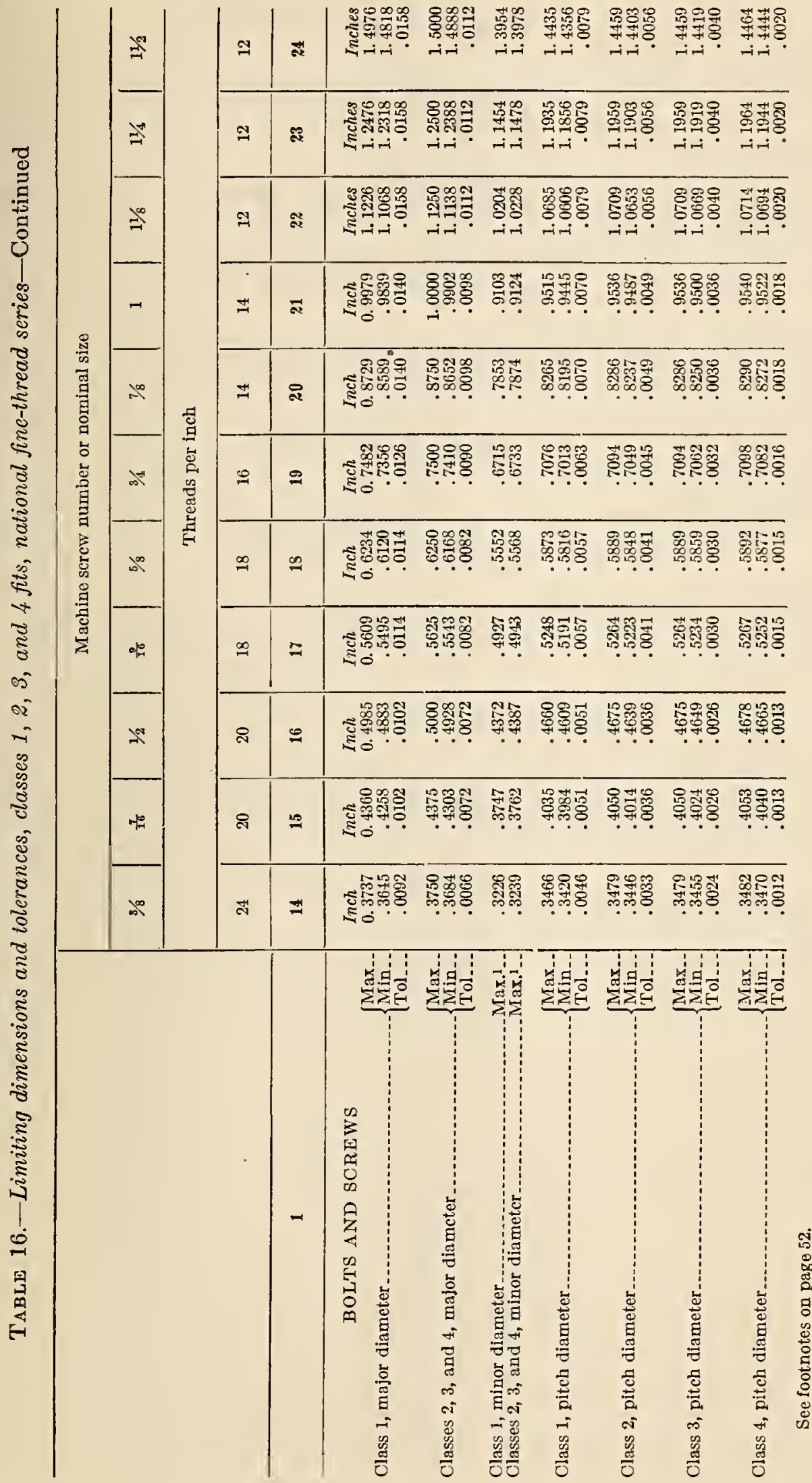




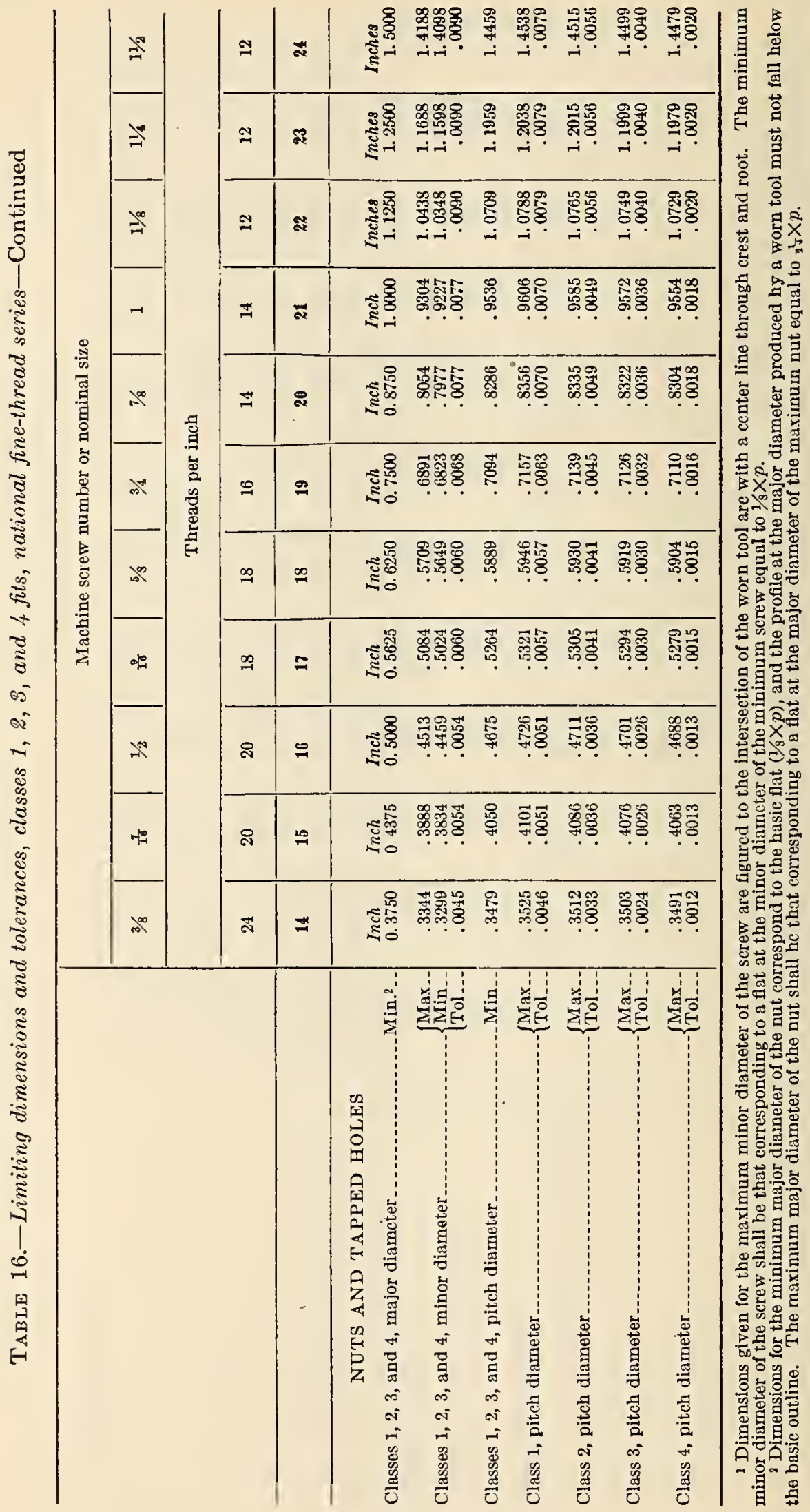




\section{SPECIFICATIONS FOR THREADING TOOLS}

The limiting sizes of the threaded product are always the essential requirements of the manufacturer and user of threaded parts. To maintain these sizes, not only the tools but also the processes employed must be suitable. So many varying factors are involved that it is not always possible to predetermine the exact sizes of the tools necessary to accomplish the required results. Variations in the nature of the material, the lubrication, the method of holding the product and tool, the sharpness of the tool, etc., all have an effect on the final results.

The effort has been made, in the following specifications, to establish sizes for various commonly used tools such that, when the tools are used with reasonable care under normal conditions, a threaded product will be produced in conformity with the product specifications. Occasions will undoubtedly arise when, as a result of peculiar combinations of circumstances, special tools will be required. ${ }^{\circ}$

\section{(a) FORM OF TOOLS FOR PRODUCING SCREWS}

Screws, or external threads are commonly produced by lathe tools, solid or adjustable dies, adjustable or opening die heads with removable chasers, thread milling cutters, threading hobs, and roller dies.

Of these, the dies, die-head chasers, and hobs are all multiple toothed, cutting in several thread spaces simultaneously, and finishing the operation at one pass. Lathe tools are ordinarily singlepointed and operate in a single thread, which is finished by repeated passes; but multiple pointed chasers for use as lathe tools are sometimes made.

All rolled threads and many cut threads are produced with dies, chasers, or hobs made with master tools, such as hobs, taps, or milling cutters. These master tools are frequently made with forming cutters or other tools, but the primary tool is always made with a single-point tool. Angle and pitch errors tend to accumulate in a series of master tools and must be carefully considered in the design and use of this single-point tool.

The tables and charts given in this section apply only to the tool used to produce the finished standard thread, and such a tool must be ussed on an axial section of the work.

1. Inspection of Tooth Outrines.-All threading tools, whether for use in a lathe, die head, thread miller, or roller, and whether single or multiple pointed, must produce the proper tooth shape on an axial section of the work. In Figure 17, for instance, the lathe threading tool is shown tipped up to the helix angle of the thread to be cut. This is sometimes done for the sake of a better cutting

An analysis of the various factors which determine the accuracy of a screw thread in production is given in Appendir 3. 
action, but necessitates a change in the tool shape, which must show an included angle of less than $60^{\circ}$, when projected on line $y-y$ of the illustration, if the resulting thread as measured on the axial section $x-x$, is to be exactly $60^{\circ}$. The ordinary thread milling cutter, set at the helix angle, and roller dies must have the same correction if accurate results are desired. In brief, the final test of accuracy in any threading tool is its ability to produce a thread of the proper axial section as defined in Section III, division 1 (a), "Specifications," of this report.

Most cutting tools for standard threads have their cutting edges in the axial plane of the work, so that the shape of those edges tends to reproduce itself on the screw thread. In forming and inspecting the cutting edges of these tools, their forms may be directly compared with standard outlines. This can be done by means of accurately formed templets, carefully applied under the microscope. A more satisfactory and practical way is to draw the desired outline on a chart to a magnification of 100 or 200 times, and then project on this chart the image of the cutting tool under inspection magnified to the corresponding degree. By this means the tool shape may be quickly compared with the standard shape to a degree of accuracy much greater than that required for commercial work. Care must be taken to use a lens system free from distortion. Optical projection machines and comparators are available for this work in commercial designs.

2. Outline for Single-Point Tool or Cutter.-In Figure 18 is shown the form of outline, measured on an axial section of the work, lequired for a single-point lathe tool or a milling cutter to produce directly a standard thread. Such a diagram, if drawn to a magnification of 100 or 200 times, will serve as a chart for projection testing. Note the shaded area at the point of the tool, within which a tool point of any shape is permissible. While sharp-cornered tools are easier to make, one with a permissible round might be more durable.

3. Odtline for Multiple-Tooth Chasers and Hobs.-In Figure 19 are given similar instructions for determining the outline of multiple-toothed chasers and hobs. The tolerance at the point or crest of the tool is the same as in Figure 18. At the roots of the teeth, however, the depth of the thread space is carried far enough to clear the crests of the screw thread being cut. This is in accordance with modern practice in threading alloy steels, which tend to tear at the crests. Relieving the chasers as shown minimizes this trouble.

It should be noted that this clearance at the crest of the screw is certain only on classes 2,3 , and 4 fits. If a class 1 screw is being cut to a minimum pitch diameter from stock of maximum major diameter size, the crests will be trimmed by the chaser. It is not possible to avoid this without carrying the grooves in the chasers nearer to a sharp bottom than is desirable. 


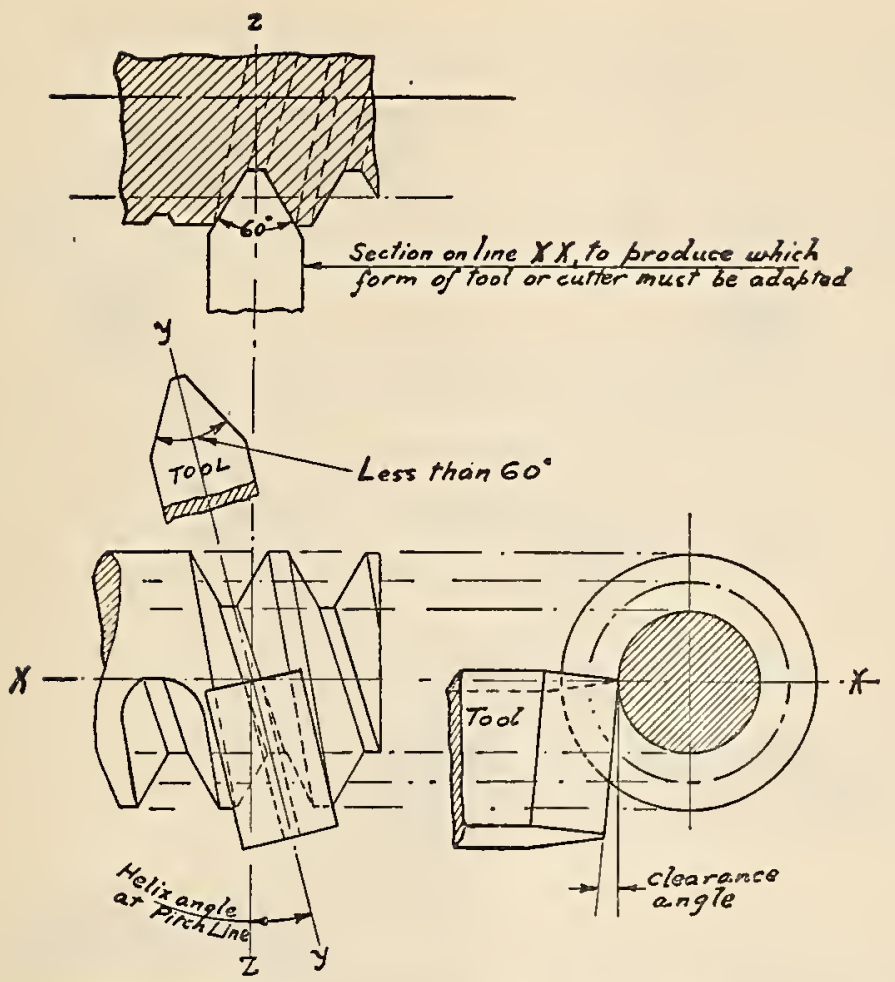

FIG. 17.-Effect on shape of tool or cutter of setting it at the helix angle

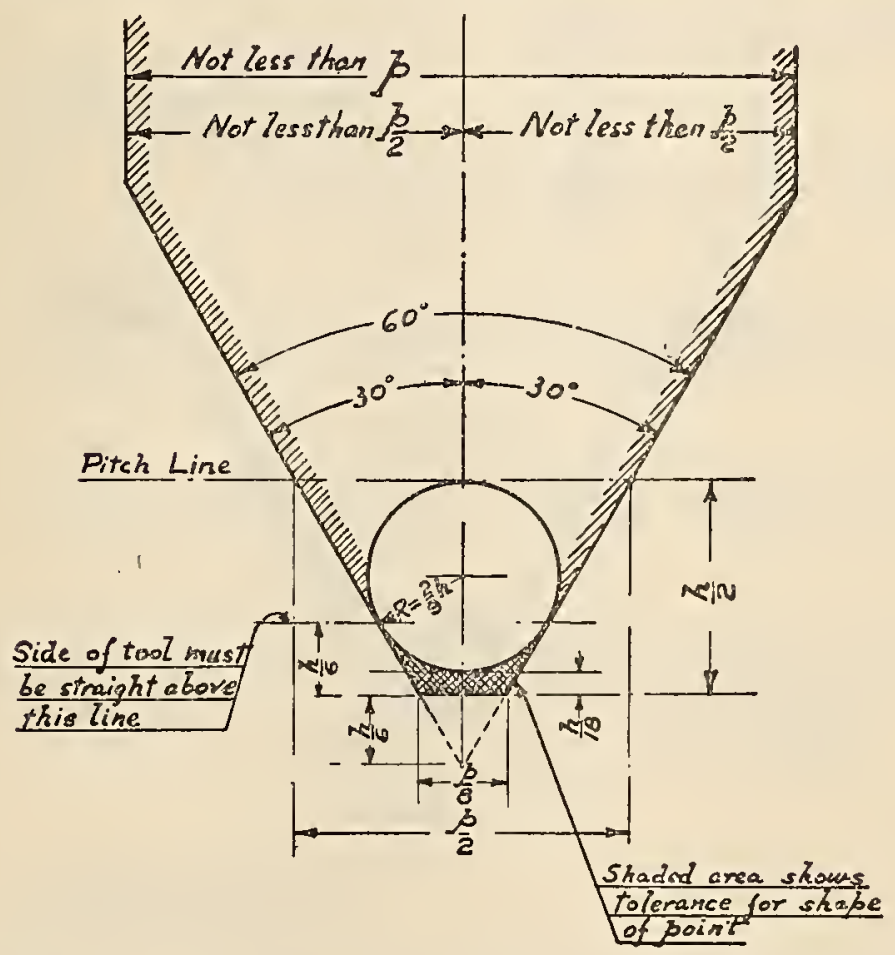

FIG. 18.- Shape for cutter or single pornt lathe tool not intended to trim crest of thread 


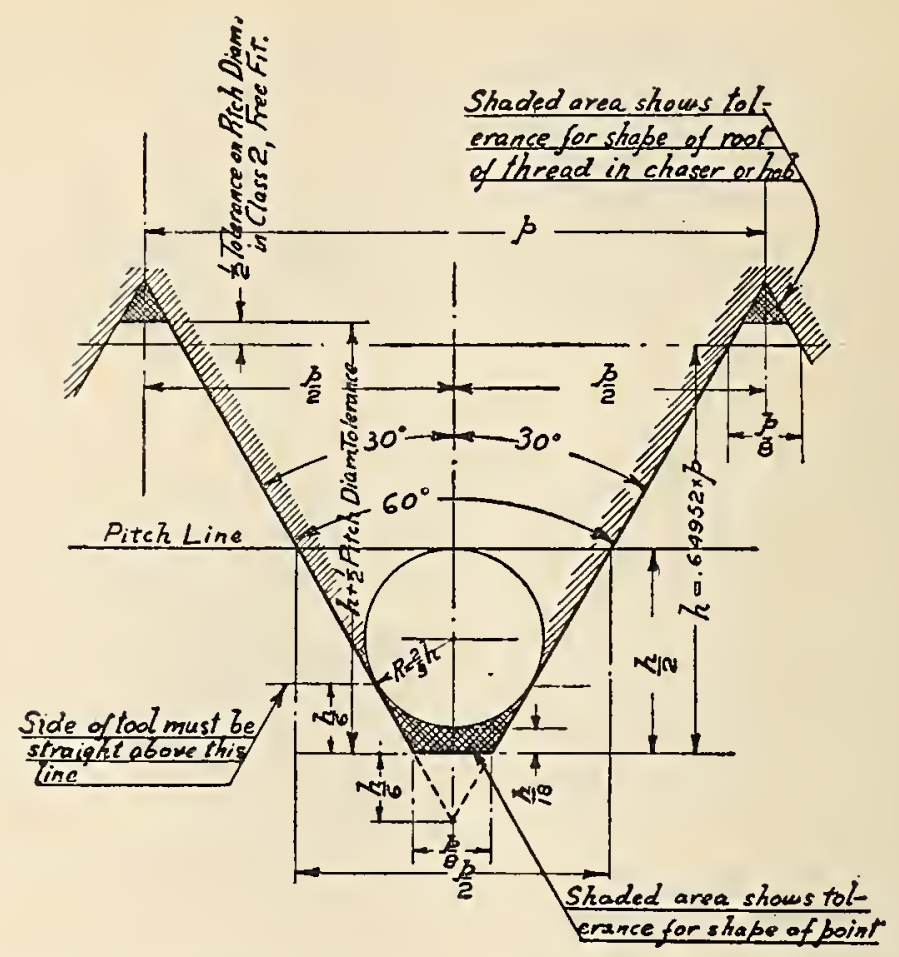

FIG. 19.-Shape for teeth of chaser or hob not intended to trim crest of thread

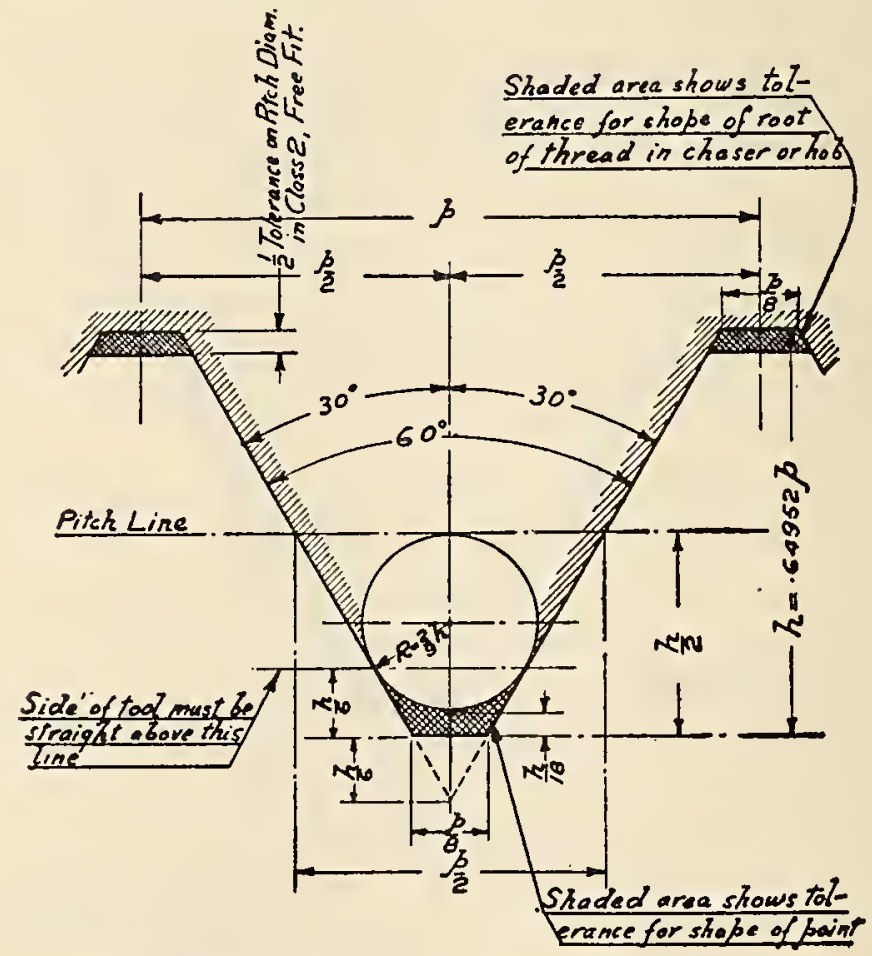

FIG. 20.- Shape for teeth of chaser or hob intended to trim crest of thread 


\begin{tabular}{|c|c|c|}
\hline 望 & $\cong$ & 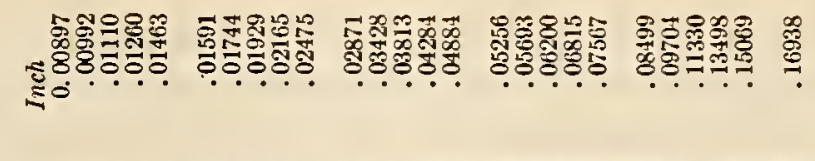 \\
\hline 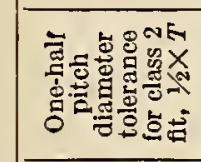 & $\cong$ & 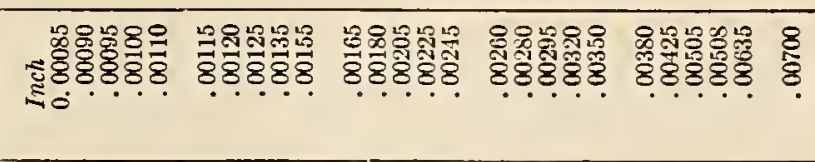 \\
\hline$\widehat{x}$ & $=$ & 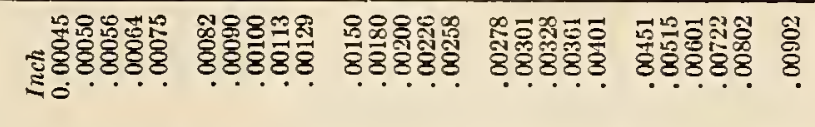 \\
\hline 蓄 & $=$ & 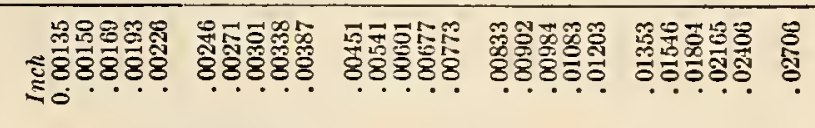 \\
\hline$\stackrel{\vec{x}}{i}$ & . & 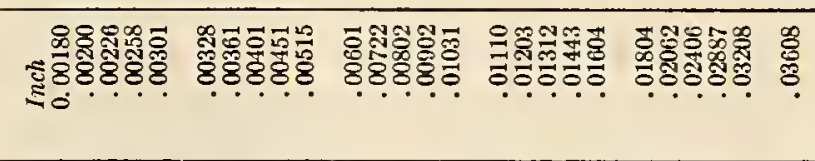 \\
\hline 齐 & $\infty$ & 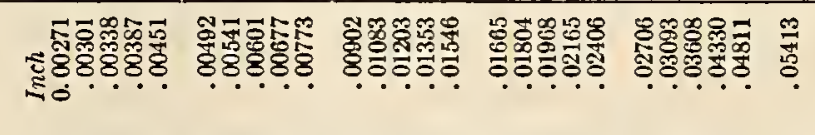 \\
\hline 离 & - & 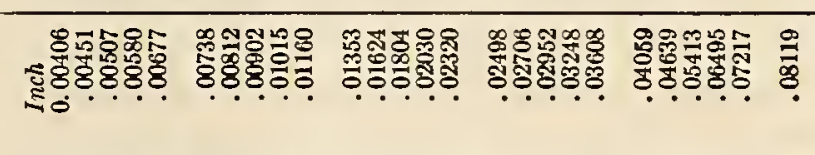 \\
\hline 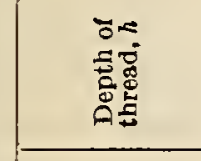 & . & 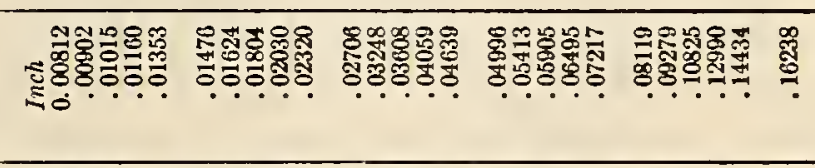 \\
\hline $\bar{x}$ & . & 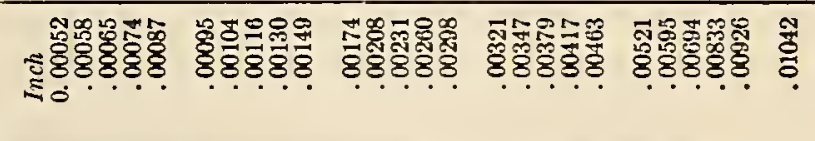 \\
\hline 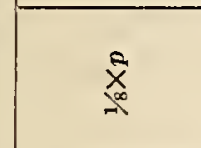 & * & 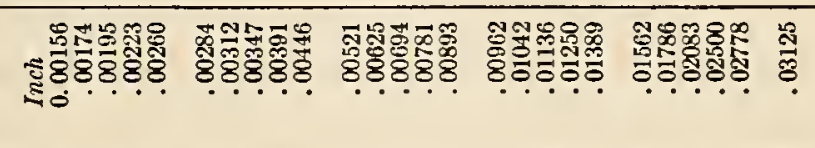 \\
\hline 音 & - & 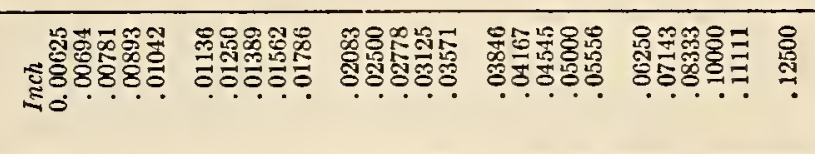 \\
\hline 章 & $\approx$ & 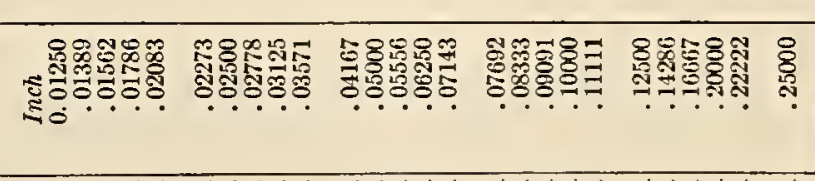 \\
\hline 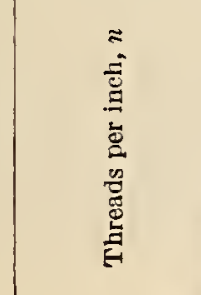 & - & \\
\hline
\end{tabular}


When it is desired to follow the older practice which permits trimming of the crests, the outline shown in Figure 20 may be used. It is somewhat easier to make than the one shown in Figure 19.

Note that the tolerance allowed on the shape of the points or crests is the same as in Figures 18 and 19. In both Figures 19 and 20 the shape of the bottom of the thread spaces may be given any curved form which can be contained within the shaded tolerance area, and still produce screw threads which will agree with the standards set up in this report.

In Table 17 are given the required dimensions for drawing the charts of Figures 18, 19, and 20 correctly for any standard pitch.

(b) TAPS

1. Tap Dimensions. - The tap dimensions given in Tables 18 to 24 , inclusive, were prepared with the cooperation of a committee of tap and die manufacturers, and represent present practice for the production of tapped holes of the classes indicated. Whether or not the following specifications for taps will prove to be those most acceptable can only be determined by their use and trial. These specifications, therefore, are tentative, and are subject to additions and revisions as experience proves necessary.

Dimensions are given for three classes of taps: First, commercial taps; second, class $\mathrm{X}$ ground taps; and third, class $\mathrm{Y}$ ground taps.

(a) Commercial taps. - The commercial taps should be found suitable, in most cases, for tapped holes made to class 1, loose-fit specifications, and for the fractional sizes of class 2 , free fit specifications. With proper selection by trial, under the actual manufacturing conditions involved, it should be found possible to use some of the commercial taps for tapped holes made to the class 2, free-fit specifications in the numbered sizes, and for the fractional sizes of class 3 , medium-fit specifications.

(b) Class $X$ ground taps. - The class $\mathrm{X}$ ground taps should be found suitable when used with reasonable care for tapped holes made to class 3 , medium-fit specifications and class 4, close-fit specifications.

(c) Class Y ground taps.-The class Y ground taps should be found suitable when used with reasonable care for tapped holes made to class 3, medium-fit specifications. The minimum size of the class $\mathrm{Y}$ tap is above the basic size to give a longer wearing life. Where accuracy requirements permit, class $\mathrm{Y}$ ground taps should be used in preference to class $\mathrm{X}$ taps because of their longer useful life.

2. Marking of Taps.--Taps shall be marked indicating the diameter, pitch, and thread series as follows (see Section II, division 2, "Symbols"). Ground taps shall be marked " $\mathrm{X}$ " or " $\mathrm{Y}$ " according to their classification. 
A 1-inch, 8-pitch tap shall be marked $1^{\prime \prime}-8-\mathrm{NC}$.

A 1-inch, 14-pitch, class $\mathrm{Y}$ ground tap shall be marked $1^{\prime \prime}-14-\mathrm{NF}-\mathrm{Y}$.

3. Shape of Cutting Edge for Taps (and other Internal Threading Tools).-In Figure 21 is shown the form of outline, measured on an axial section of the work, required for a tap or other internal threading tool intended for cutting threads of national form. These outlines are the same for all pitches and classes of fit. Such a diagram, if drawn to a magnification of 100 or 200 times, will serve as a chart for projection testing. Note the shaded area at the

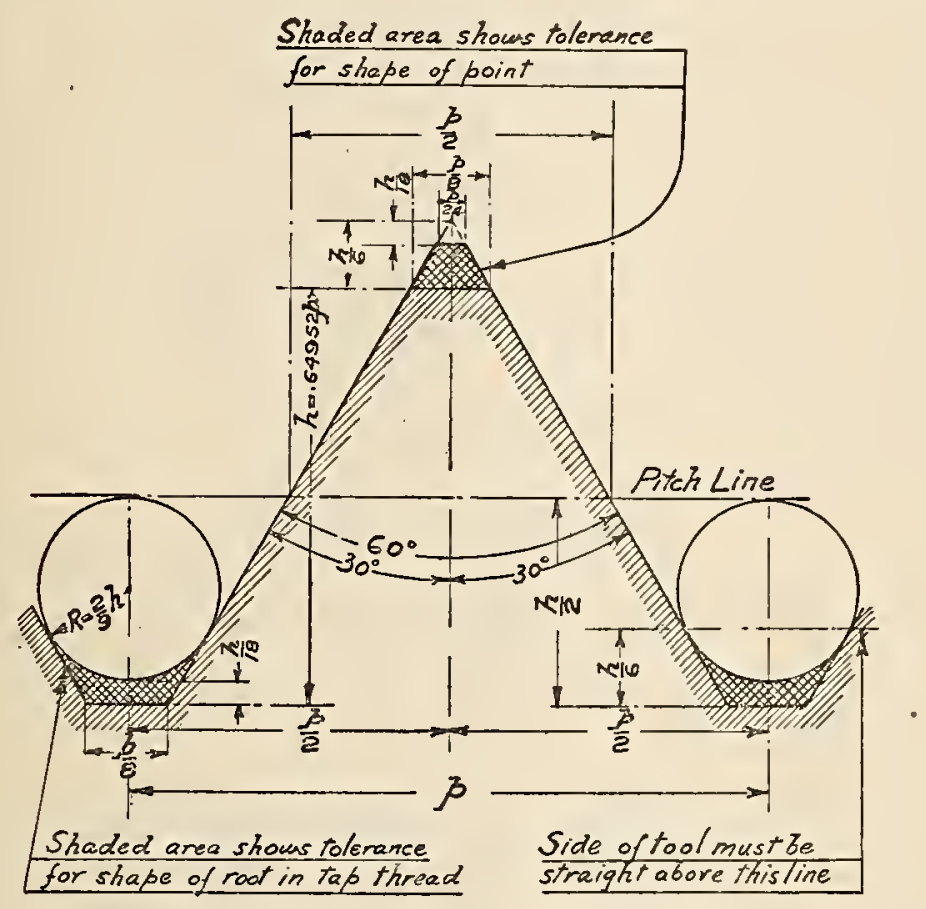

FIG. 21.-Shape for teeth of tap or internal thread-chasing tool

point of the tool, within which a tool point of any shape is permissible. Also note the shaded area at the root of the thread which shows permissible variations in tooth outline at this point.

While sharp-cornered tools are easier to make, one with a permissible round at the point might be more durable. In any event, the maker of the tap or other tool should keep the outline well away from the lower part of the permissible. area, represented by the basic flat of $1 / 8 \times p$. The danger here is that after a few holes have been tapped the corners will be rounded over so that the tapped hole rejects the basic gage.

Table 17 gives the necessary dimensions for drawing the diagram shown in Figure 21 for any standard pitch. 
TABLE 18.-Dimensions of hand taps

\begin{tabular}{|c|c|c|c|c|c|c|c|c|}
\hline \multirow{2}{*}{$\begin{array}{l}\text { Nominal size of } \\
\text { tap in inches }\end{array}$} & \multicolumn{2}{|c|}{ Length over all } & \multicolumn{2}{|c|}{ Length of thread } & \multicolumn{2}{|c|}{ Diameter of shank } & \multicolumn{2}{|c|}{ Size of square } \\
\hline & Nominal & $\begin{array}{l}\text { Toler- } \\
\text { ance }\end{array}$ & Nominal & $\begin{array}{l}\text { Toler- } \\
\text { ance }\end{array}$ & Nominal & $\begin{array}{l}\text { Toler- } \\
\text { ance }\end{array}$ & Nominal & $\begin{array}{l}\text { Toler- } \\
\text { ance }\end{array}$ \\
\hline 1 & 2 & 3 & 4 & 5 & 6 & 7 & 8 & 9 \\
\hline & 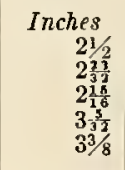 & $\begin{array}{l}\text { Inch } \\
\pm \frac{3}{31} \\
\pm \frac{1}{33} \\
\pm \frac{1}{33} \\
\pm \frac{1}{32} \\
\pm \frac{1}{32} \\
\pm \frac{13}{32}\end{array}$ & $\begin{array}{c}\text { Inches } \\
1 \\
11 / 8 \\
11 / 4 \\
11 \\
1 \frac{1}{16} \\
1 \frac{12}{32}\end{array}$ & 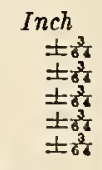 & $\begin{array}{c}\text { Inches } \\
0.2530 \\
.3155 \\
.3785 \\
.3232 \\
.3667\end{array}$ & $\begin{array}{l}\text { Inch } \\
-0.005 \\
=.005 \\
=.005 \\
=.005 \\
-.005\end{array}$ & $\begin{array}{c}\text { Inches } \\
0.1897 \\
.2366 \\
.2839 \\
.2424 \\
.2750\end{array}$ & $\begin{array}{l}\text { Inch } \\
=0.004 \\
=.004 \\
=.004 \\
=.004 \\
-.004\end{array}$ \\
\hline & $\begin{array}{l}3 \frac{19}{32} \\
3115 \\
41 / 4 \\
412 \\
51 / 8\end{array}$ & $\begin{array}{l} \pm \frac{1}{32} \\
\pm \frac{1}{33} \\
\pm \frac{1}{33} \\
\pm \frac{3}{33} \\
\pm \frac{3}{32}\end{array}$ & $\begin{array}{l}1 \frac{21}{3} \\
1_{1 \frac{13}{16}} \\
2 \\
2 \frac{3}{32} \\
2 \frac{3}{1 / 2}\end{array}$ & 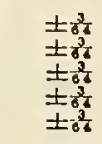 & $\begin{array}{r}.4292 \\
4796 \\
.5900 \\
.6973 \\
.8000\end{array}$ & $\begin{array}{l}=.005 \\
=.005 \\
=.005 \\
=.005 \\
-.005\end{array}$ & $\begin{array}{l}.3219 \\
.3597 \\
.4425 \\
.5230 \\
.6000\end{array}$ & $\begin{array}{l}=.006 \\
=.006 \\
=.006 \\
=.006 \\
-.006\end{array}$ \\
\hline 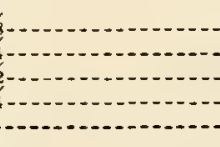 & $\begin{array}{l}5 \frac{7}{16} \\
53 / 4 \\
63 / 8 \\
7 \\
73 / 8\end{array}$ & $\begin{array}{l} \pm \frac{1}{16} \\
\pm 1 \frac{1}{16} \\
\pm \frac{1}{16} \\
\pm \frac{1}{16} \\
\pm \frac{16}{16}\end{array}$ & $\begin{array}{l}2 \frac{1}{18} \\
2 \frac{3}{18} \\
3 \\
3 \frac{9}{18} \\
3 \frac{18}{18}\end{array}$ & 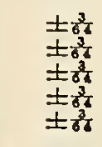 & $\begin{array}{l}.8965 \\
1.0215 \\
1.2333 \\
1.4300 \\
1.6445\end{array}$ & $\begin{array}{l}=.007 \\
=.007 \\
=.007 \\
=.007 \\
=.007\end{array}$ & $\begin{array}{r}.6724 \\
.7661 \\
.9250 \\
1.0725 \\
1.2334\end{array}$ & $\begin{array}{r}=.008 \\
=.008 \\
=.008 \\
=.008 \\
-.008\end{array}$ \\
\hline & $\begin{array}{l}81 / 4 \\
83 / 4 \\
91 / 4 \\
93 / 6\end{array}$ & $\begin{array}{l} \pm 18 \\
\pm 18 \\
\pm 16 \\
\pm 18 \\
\pm \frac{1}{16}\end{array}$ & $\begin{array}{l}3 \frac{1}{18} \\
4 \\
4 \\
4 \frac{18}{18}\end{array}$ & $\begin{array}{l} \pm \frac{3}{86} \\
\pm \frac{3}{86} \\
\pm \frac{3}{64} \\
\pm \frac{3}{66}\end{array}$ & $\begin{array}{l}1.8944 \\
2.1000 \\
2.3500 \\
2.5429\end{array}$ & $\begin{array}{r}-.009 \\
=.009 \\
-.009 \\
-.009\end{array}$ & $\begin{array}{l}1.4208 \\
1.5750 \\
1.7625 \\
1.9072\end{array}$ & $\begin{array}{r}-.010 \\
=.010 \\
=.010 \\
-.0: 0\end{array}$ \\
\hline
\end{tabular}

TABLE 19.-Dimensions and tolerances for commercial taps, national coarse-thread series

\begin{tabular}{|c|c|c|c|c|c|c|c|c|}
\hline \multirow[b]{2}{*}{ Sizes } & \multirow[b]{2}{*}{$\begin{array}{l}\text { Threads } \\
\text { per inch }\end{array}$} & \multicolumn{2}{|c|}{ Major diameter } & \multicolumn{3}{|c|}{ Pitch diameter } & \multicolumn{2}{|c|}{ Basic dlameter } \\
\hline & & $\begin{array}{l}\text { Mini- } \\
\text { mum }\end{array}$ & $\begin{array}{c}\text { Maxi- } \\
\text { mum- } \\
\text { measured } \\
\text { pitch } \\
\text { diameter } \\
\text { plus }\end{array}$ & $\begin{array}{l}\text { Mini- } \\
\text { mum }\end{array}$ & $\begin{array}{l}\text { Toler- } \\
\text { ance }\end{array}$ & $\begin{array}{l}\text { Maxi- } \\
\text { mum } 1\end{array}$ & Major & Pitcl \\
\hline 1 & $\cdot 2$ & 3 & 1 & 5 & 6 & 7 & 8 & 9 \\
\hline $\begin{array}{l}1 \\
2 \\
3 \\
4\end{array}$ & $\begin{array}{l}64 \\
56 \\
48 \\
40 \\
40\end{array}$ & $\begin{array}{c}\text { Inches } \\
0.0740 \\
.0870 \\
.1000 \\
.1130 \\
.1260\end{array}$ & $\begin{array}{l}\text { Inch } \\
0.0124 \\
.0142 \\
.0165 \\
.0198 \\
.0198\end{array}$ & $\begin{array}{r}\text { Inches } \\
0.0634 \\
.0749 \\
.0860 \\
.0963 \\
.1093\end{array}$ & $\begin{array}{l}\text { Inch } \\
0.0010 \\
.0010 \\
.0010 \\
.0015 \\
.0015\end{array}$ & $\begin{array}{r}\text { Inch } s \\
0.0644 \\
.0759 \\
.0870 \\
.0978 \\
.1108\end{array}$ & $\begin{array}{r}\text { Inches } \\
0.073 \\
.086 \\
.099 \\
.112 \\
.125\end{array}$ & $\begin{array}{r}\text { Inches } \\
0.0629 \\
.0744 \\
.0855 \\
.0959 \\
.1088\end{array}$ \\
\hline $\begin{array}{l}6 \\
8 \ldots \ldots \\
10 \ldots \\
12 \ldots\end{array}$ & $\begin{array}{l}32 \\
32 \\
24 \\
24\end{array}$ & $\begin{array}{l}.1390 \\
.1650 \\
.1910 \\
.2170\end{array}$ & $\begin{array}{l}.0248 \\
.0248 \\
.0331 \\
.0331\end{array}$ & $\begin{array}{l}.1182 \\
.1442 \\
.1634 \\
.1894\end{array}$ & $\begin{array}{l}.0015 \\
.0015 \\
.0015 \\
.0015\end{array}$ & $\begin{array}{l}.1197 \\
.1457 \\
.1649 \\
.1909\end{array}$ & $\begin{array}{l}.138 \\
.164 \\
.190 \\
.216\end{array}$ & $\begin{array}{r}.1177 \\
.1437 \\
.1629 \\
.1889\end{array}$ \\
\hline $\begin{array}{l}1 / 4 \ldots \\
\frac{5}{16} \ldots \ldots \\
3 / 8 \\
\frac{7}{16} \\
1 / 2 \ldots \ldots\end{array}$ & $\begin{array}{l}20 \\
18 \\
16 \\
14 \\
13\end{array}$ & $\begin{array}{l}.2510 \\
.3135 \\
.3760 \\
.4385 \\
.5010\end{array}$ & $\begin{array}{l}.0397 \\
.0441 \\
.0496 \\
.0567 \\
.0611\end{array}$ & $\begin{array}{l}.2180 \\
.2769 \\
.3349 \\
.3916 \\
.4505\end{array}$ & $\begin{array}{l}.0020 \\
.0020 \\
.0020 \\
.0025 \\
.0025\end{array}$ & $\begin{array}{r}.2200 \\
.2789 \\
3369 \\
.3941 \\
.4530\end{array}$ & $\begin{array}{r}.2500 \\
.3125 \\
.3750 \\
.4375 \\
.5000\end{array}$ & $\begin{array}{r}.2175 \\
.2761 \\
.3341 \\
.3911 \\
.4500\end{array}$ \\
\hline $\begin{array}{l}\frac{9}{16}-\ldots \\
5 / 8 \ldots \ldots \\
3 / 1 \\
7 / 8 \ldots \\
1 \ldots\end{array}$ & $\begin{array}{r}12 \\
11 \\
10 \\
9 \\
8\end{array}$ & $\begin{array}{r}.5635 \\
.6260 \\
.7510 \\
.8760 \\
1.0010\end{array}$ & $\begin{array}{l}.0662 \\
.0722 \\
.0794 \\
.0882 \\
.0992\end{array}$ & $\begin{array}{l}.5089 \\
.5665 \\
.6855 \\
.8033 \\
.9193\end{array}$ & $\begin{array}{l}.0025 \\
.0025 \\
.0030 \\
.0030 \\
.0030\end{array}$ & $\begin{array}{l}.5114 \\
.5690 \\
.6885 \\
.8063 \\
.9223\end{array}$ & $\begin{array}{r}.5625 \\
.6250 \\
.7500 \\
.8750 \\
1.0000\end{array}$ & $\begin{array}{l}.5084 \\
.5660 \\
.6850 \\
.8028 \\
.9188\end{array}$ \\
\hline $\begin{array}{l}11 / 8 \ldots \\
11 / 4 \ldots \\
11 / 2 \ldots \\
13 / 4 \ldots \\
2 \ldots\end{array}$ & $\begin{array}{l}7 \\
7 \\
6 \\
5 \\
41 / 2\end{array}$ & $\begin{array}{l}1.1265 \\
1.2515 \\
1.5015 \\
1.7515 \\
2.0015\end{array}$ & $\begin{array}{l}.1134 \\
.1134 \\
.1323 \\
.1588 \\
.1764\end{array}$ & $\begin{array}{l}1.0327 \\
1.1577 \\
1.3922 \\
1.6211 \\
1.8567\end{array}$ & $\begin{array}{l}.0035 \\
.0035 \\
.0035 \\
.0040 \\
.0045\end{array}$ & $\begin{array}{l}1.0362 \\
1.1612 \\
1.3957 \\
1.6251 \\
1.8612\end{array}$ & $\begin{array}{l}1.1250 \\
1.2500 \\
1.5000 \\
1.7500 \\
2.0000\end{array}$ & $\begin{array}{l}1.0322 \\
1.1572 \\
1.3917 \\
1.6201 \\
1.8557\end{array}$ \\
\hline $\begin{array}{l}21 / 4 \\
21 / 2 \\
23 / 4 \\
3\end{array}$ & $\begin{array}{l}41 / 2 \\
4 \\
4 \\
4\end{array}$ & $\begin{array}{l}2.2520 \\
2.5020 \\
2.7520 \\
3.0020\end{array}$ & $\begin{array}{l}.1764 \\
.1985 \\
.1985 \\
.1985\end{array}$ & $\begin{array}{l}\text { 2. } 1067 \\
2.3386 \\
2.5886 \\
2.8386\end{array}$ & $\begin{array}{l}.0045 \\
.0050 \\
.0050 \\
.0050\end{array}$ & $\begin{array}{l}2.1112 \\
2.3436 \\
2.5936 \\
2.8436\end{array}$ & $\begin{array}{l}2.2500 \\
2.5000 \\
2.7500 \\
\text { 3. } 0000\end{array}$ & $\begin{array}{l}2.1057 \\
2.3376 \\
2.5876 \\
2.8376\end{array}$ \\
\hline
\end{tabular}

Note.-Maximum lead error $= \pm 0.003$ inch in 1 inch of thread.

1 If a tap should be of maximum diameter and contain the maximum lead error, it mas not produco tapped holes within the specifled tolerances for the product. 
TABLE 20.-Dimensions and tolerances for commercial taps, national fine-thread series

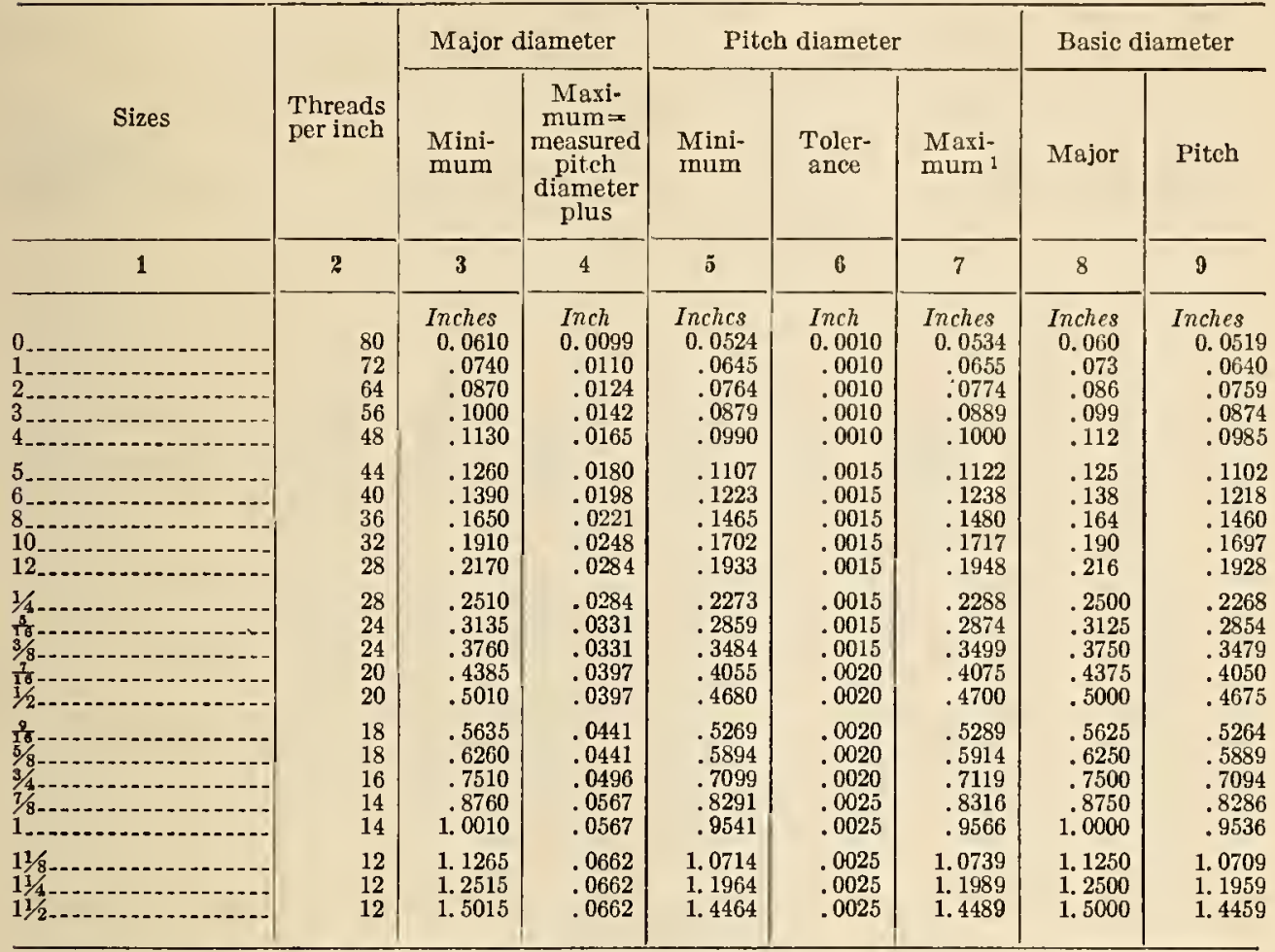

Note. -Maximum lead error $= \pm 0.003$ inch in 1 inch of thread.

1 If a tap should be of maximum diameter and contain the maximum lead error, it may not produce tapped boles within the specified tolerances for the product.

TABLE 21.-Dimensions and tolerances for class $X$ ground taps, national coarsethread series

\begin{tabular}{|c|c|c|c|c|c|c|c|c|c|}
\hline \multirow[b]{2}{*}{ Sizes } & \multirow[b]{2}{*}{$\begin{array}{l}\text { Threads } \\
\text { per inch }\end{array}$} & \multicolumn{2}{|c|}{ Major diameter } & \multicolumn{3}{|c|}{ Pitch diameter } & \multicolumn{2}{|c|}{ Basic diameter } & \multirow[b]{2}{*}{$\begin{array}{l}\text { Toler- } \\
\text { ance on } \\
\text { half angle } \\
\text { of thread }\end{array}$} \\
\hline & & $\begin{array}{l}\text { Mini- } \\
\text { mum }\end{array}$ & $\begin{array}{c}\text { Maxi- } \\
\text { mum= } \\
\text { measured } \\
\text { pitch } \\
\text { diameter } \\
\text { plus }\end{array}$ & $\begin{array}{l}\text { Mini- } \\
\text { mum }\end{array}$ & $\begin{array}{l}\text { Toler- } \\
\text { ance }\end{array}$ & $\begin{array}{l}\text { Maxi- } \\
\text { mum }\end{array}$ & Major & Pitch & \\
\hline 1 & 2 & 3 & 4 & 5 & 6 & 7 & 8 & 9 & 10 \\
\hline & $\begin{array}{l}20 \\
18 \\
16 \\
14 \\
13\end{array}$ & $\begin{array}{c}\text { Inches } \\
0.2520 \\
.3145 \\
.3770 \\
.4400 \\
.5025\end{array}$ & $\begin{array}{l}\text { Inch } \\
0.0397 \\
.0441 \\
.0496 \\
.0567 \\
.0611\end{array}$ & $\begin{array}{c}\text { Inches } \\
0.2175 \\
.2764 \\
.3344 \\
.3911 \\
.4500\end{array}$ & $\begin{array}{l}\text { Inch } \\
0.0008 \\
.0008 \\
.0008 \\
.0009 \\
.0009\end{array}$ & $\begin{array}{c}\text { Inches } \\
0.2183 \\
.2772 \\
.3352 \\
.3920 \\
.4509\end{array}$ & $\begin{array}{c}\text { Inches } \\
0.2500 \\
.3125 \\
.3750 \\
.4375 \\
.5000\end{array}$ & $\begin{array}{c}\text { Inches } \\
0.2175 \\
.2764 \\
.3344 \\
.3911 \\
.4500\end{array}$ & $\begin{array}{cc}\text { Deg. } & \text { Min } \\
0 & 15 \\
0 & 15 \\
0 & 15 \\
0 & 15 \\
0 & 14\end{array}$ \\
\hline & $\begin{array}{r}12 \\
11 \\
10 \\
. \quad 9 \\
8\end{array}$ & $\begin{array}{r}.5650 \\
.6275 \\
.7530 \\
.8780 \\
1.0030\end{array}$ & $\begin{array}{l}.0662 \\
.0722 \\
.0794 \\
.0882 \\
.0992\end{array}$ & $\begin{array}{l}.5084 \\
.5660 \\
.6850 \\
.8028 \\
.9188\end{array}$ & $\begin{array}{l}.0010 \\
.0010 \\
.0011 \\
.0012 \\
.0013\end{array}$ & $\begin{array}{l}.5094 \\
.5670 \\
.6861 \\
.8040 \\
.9201\end{array}$ & $\begin{array}{r}.5625 \\
.6250 \\
.7500 \\
.8750 \\
1.0000\end{array}$ & $\begin{array}{l}.5084 \\
.5660 \\
.6850 \\
.8028 \\
.9188\end{array}$ & $\begin{array}{l}0 \\
0 \\
0 \\
0 \\
0\end{array}$ \\
\hline & \begin{tabular}{l|}
7 \\
7 \\
6 \\
5 \\
$41 / 2$
\end{tabular} & $\begin{array}{l}1.1290 \\
1.2540 \\
1.5040 \\
1.7550 \\
2.0050\end{array}$ & $\begin{array}{l}.1134 \\
.1134 \\
.1323 \\
.1588 \\
.1764\end{array}$ & $\begin{array}{l}1.0322 \\
1.1572 \\
1.3917 \\
1.6201 \\
1.8557\end{array}$ & $\begin{array}{l}.0014 \\
.0014 \\
.0015 \\
.0015 \\
.0015\end{array}$ & $\begin{array}{l}1.0336 \\
1.1586 \\
1.3932 \\
1.6216 \\
1.8572\end{array}$ & $\begin{array}{l}1.1250 \\
1.2500 \\
1.5000 \\
1.7500 \\
2.0000\end{array}$ & $\begin{array}{l}1.0322 \\
1.1572 \\
1.3917 \\
1.6201 \\
1.8557\end{array}$ & $\begin{array}{l}0 \\
0 \\
0 \\
0 \\
0\end{array}$ \\
\hline & $\begin{array}{l}41 / 2 \\
4 \\
4 \\
4\end{array}$ & $\begin{array}{l}2.2560 \\
2.5060 \\
2.7570 \\
3.0070\end{array}$ & $\begin{array}{l}.1764 \\
.1985 \\
.1985 \\
.1985\end{array}$ & $\begin{array}{l}\text { 2. } 1057 \\
\text { 2. } 3376 \\
\text { 2. } 5876 \\
2.8376\end{array}$ & $\begin{array}{l}.0015 \\
.9015 \\
.0015 \\
.0015\end{array}$ & $\begin{array}{l}2.1072 \\
2.3391 \\
2.5891 \\
2.8391\end{array}$ & $\begin{array}{l}2.2500 \\
2.5000 \\
2.7500 \\
3.0000\end{array}$ & $\begin{array}{l}2.1057 \\
2.3376 \\
2.5876 \\
2.8376\end{array}$ & $\begin{array}{l}0 \\
0 \\
0 \\
0\end{array}$ \\
\hline
\end{tabular}

Note. - Maximum lead error $= \pm 0.0005$ inch in 1 inch of thread.

$16802^{\circ}-25 \dagger-5$ 
TABLE 22.-Dimensions and tolerances for class $X$ ground taps, national fine-thread series

\begin{tabular}{|c|c|c|c|c|c|c|c|c|c|}
\hline \multirow[b]{2}{*}{ Sizes } & \multirow[b]{2}{*}{$\begin{array}{l}\text { Threads } \\
\text { per inch }\end{array}$} & \multicolumn{2}{|c|}{ Major diameter } & \multicolumn{3}{|c|}{ Pitch diameter } & \multicolumn{2}{|c|}{ Basic diameter } & \multirow[b]{2}{*}{$\begin{array}{c}\text { Toler- } \\
\text { ance on } \\
\text { half angle } \\
\text { of thread }\end{array}$} \\
\hline & & $\begin{array}{l}\text { Mini- } \\
\text { mum }\end{array}$ & $\begin{array}{c}\text { Maxi- } \\
\text { mum= } \\
\text { measured } \\
\text { pitch } \\
\text { diameter } \\
\text { plus }\end{array}$ & $\begin{array}{l}\text { Mini- } \\
\text { mum }\end{array}$ & $\begin{array}{l}\text { Toler- } \\
\text { ance }\end{array}$ & $\begin{array}{l}\text { Maxi- } \\
\text { mum }\end{array}$ & Major & Pitch & \\
\hline 1 & 2 & 3 & 4 & 5 & 6 & 7 & 8 & 9 & 10 \\
\hline & $\begin{array}{l}28 \\
24 \\
24 \\
20 \\
20\end{array}$ & $\begin{array}{c}\text { Inches } \\
0.2520 \\
.3145 \\
.3770 \\
.4400 \\
.5025\end{array}$ & $\begin{array}{l}\text { Inch } \\
0.0284 \\
.0831 \\
.0331 \\
.0397 \\
.0397\end{array}$ & $\begin{array}{c}\text { Inches } \\
0.2268 \\
.2854 \\
.3479 \\
.4050 \\
.4675\end{array}$ & $\begin{array}{l}\text { Inch } \\
0.0008 \\
.0008 \\
.0008 \\
.0008 \\
.0008\end{array}$ & $\begin{array}{c}\text { Inches } \\
0.2276 \\
.2862 \\
.3487 \\
.4058 \\
.4683\end{array}$ & $\begin{array}{c}\text { Inches } \\
0.2500 \\
.3125 \\
.3750 \\
.4375 \\
.5000\end{array}$ & $\begin{array}{c}\text { Inches } \\
0.2268 \\
.2854 \\
.3479 \\
.4050 \\
.4675\end{array}$ & $\begin{array}{cc}\text { Deg. } & \text { Min } \\
0 & 1 \\
0 & 1 \\
0 & 1 \\
0 & 1 \\
0 & 1\end{array}$ \\
\hline & $\begin{array}{l}18 \\
18 \\
16 \\
14 \\
14\end{array}$ & $\begin{array}{r}.5650 \\
.6275 \\
.7530 \\
.8780 \\
1.0030\end{array}$ & $\begin{array}{l}.0441 \\
.0441 \\
.0496 \\
.0567 \\
.0567\end{array}$ & $\begin{array}{l}.5264 \\
.5889 \\
.7094 \\
.8286 \\
.9536\end{array}$ & $\begin{array}{l}.0008 \\
.0008 \\
.0008 \\
.0009 \\
.0009\end{array}$ & $\begin{array}{l}.5272 \\
.5897 \\
.7102 \\
.8295 \\
.9545\end{array}$ & $\begin{array}{r}.5625 \\
.6250 \\
.7500 \\
.8750 \\
1.0000\end{array}$ & $\begin{array}{l}.5264 \\
.5889 \\
.7094 \\
.8286 \\
.9536\end{array}$ & $\begin{array}{l}0 \\
0 \\
0 \\
0 \\
0\end{array}$ \\
\hline & $\begin{array}{l}12 \\
12 \\
12\end{array}$ & $\begin{array}{l}\text { 1. } 1290 \\
1.2540 \\
\text { 1. } 5040\end{array}$ & $\begin{array}{l}.0662 \\
.0662 \\
.0662\end{array}$ & $\begin{array}{l}\text { 1. } 0709 \\
1.1959 \\
1.4459\end{array}$ & $\begin{array}{l}.0010 \\
.0010 \\
.0010\end{array}$ & $\begin{array}{l}\text { 1. } 0719 \\
\text { 1. } 1969 \\
\text { 1. } 4469\end{array}$ & $\begin{array}{l}1.1250 \\
1.2500 \\
1.5000\end{array}$ & $\begin{array}{l}1.0709 \\
1.1959 \\
1.4459\end{array}$ & $\begin{array}{l}0 \\
0 \\
0\end{array}$ \\
\hline
\end{tabular}

Note. - Maximum lead error $= \pm 0.0005$ inch in 1 inch of thread.

TABLE 23.-Dimensions and tolerances for class $Y$ ground taps, national coarsethread series

\begin{tabular}{|c|c|c|c|c|c|c|c|c|c|c|}
\hline \multirow[b]{2}{*}{ Sizes } & \multirow[b]{2}{*}{$\begin{array}{l}\text { Threads } \\
\text { per inch }\end{array}$} & \multicolumn{2}{|c|}{ Major diameter } & \multicolumn{3}{|c|}{ Pitch diameter } & \multicolumn{2}{|c|}{ Basic diameter } & \multirow{2}{*}{\multicolumn{2}{|c|}{$\begin{array}{c}\text { Toler- } \\
\text { ance on } \\
\text { half angle } \\
\text { of thread }\end{array}$}} \\
\hline & & $\begin{array}{l}\text { Mini- } \\
\text { mum }\end{array}$ & $\begin{array}{c}\text { Maxi- } \\
\text { mum= } \\
\text { measured } \\
\text { pitch } \\
\text { diameter } \\
\text { plus }\end{array}$ & $\begin{array}{l}\text { Mini- } \\
\text { mum }\end{array}$ & $\begin{array}{l}\text { Toler- } \\
\text { ance }\end{array}$ & $\begin{array}{l}\text { Maxi- } \\
\text { mum }\end{array}$ & Major & Pitch & & \\
\hline 1 & 2 & 3 & 4 & 5 & 6 & 7 & 8 & 9 & 10 & \\
\hline 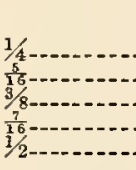 & $\begin{array}{l}20 \\
18 \\
16 \\
14 \\
13\end{array}$ & $\begin{array}{r}\text { Inches } \\
0.2525 \\
.3150 \\
.3776 \\
.4407 \\
.5032\end{array}$ & $\begin{array}{l}\text { Inch } \\
0.0397 \\
.0441 \\
.0496 \\
.0567 \\
.0611\end{array}$ & $\begin{array}{c}\text { Inches } \\
0.2180 \\
.2769 \\
.3350 \\
.3918 \\
.4507\end{array}$ & $\begin{array}{l}\text { Inch } \\
0.0010 \\
.0010 \\
.0011 \\
.0012 \\
.0013\end{array}$ & $\begin{array}{r}\text { Inches } \\
0.2190 \\
.2779 \\
.3361 \\
.3930 \\
.4520\end{array}$ & $\begin{array}{c}\text { Inches } \\
0.2500 \\
.3125 \\
.3750 \\
.4375 \\
.5000\end{array}$ & $\begin{array}{c}\text { Inches } \\
0.2175 \\
.2764 \\
.3344 \\
.3911 \\
.4500\end{array}$ & $\begin{array}{c}\text { Deg. } M \\
0 \\
0 \\
0 \\
0 \\
0\end{array}$ & $\begin{array}{r}\text { rin. } \\
20 \\
20 \\
19 \\
19 \\
18\end{array}$ \\
\hline $\begin{array}{l}\frac{9}{166}-\ldots \\
5 / 8-\ldots \\
3 / 4 \\
7 / 8 \\
1\end{array}$ & $\begin{array}{r}12 \\
11 \\
10 \\
9 \\
8\end{array}$ & $\begin{array}{r}.5658 \\
.6283 \\
.7539 \\
.8790 \\
1.0041\end{array}$ & $\begin{array}{l}.0662 \\
.0722 \\
.0794 \\
.0882 \\
.0992\end{array}$ & $\begin{array}{l}.5092 \\
.5668 \\
.6859 \\
.8038 \\
.9199\end{array}$ & $\begin{array}{l}.0014 \\
.0014 \\
.0014 \\
.0015 \\
.0016\end{array}$ & $\begin{array}{l}.5106 \\
.5682 \\
.6873 \\
.8053 \\
.9215\end{array}$ & $\begin{array}{r}.5625 \\
.6250 \\
.7500 \\
.8750 \\
1.0000\end{array}$ & $\begin{array}{l}.5084 \\
.5660 \\
.6850 \\
.8028 \\
.9188\end{array}$ & $\begin{array}{l}0 \\
0 \\
0 \\
0 \\
0\end{array}$ & $\begin{array}{l}18 \\
18 \\
17 \\
17 \\
17\end{array}$ \\
\hline $\begin{array}{l}11 / 8 \\
11 / 4 \\
11 / 2 \\
13 / 4 \\
2\end{array}$ & $\begin{array}{l}7 \\
7 \\
6 \\
5 \\
41 / 2\end{array}$ & $\begin{array}{l}1.1302 \\
1.2552 \\
1.5054 \\
1.7566 \\
2.0068\end{array}$ & $\begin{array}{l}.1134 \\
.1134 \\
.1323 \\
.1588 \\
.1764\end{array}$ & $\begin{array}{l}1.0334 \\
1.1584 \\
1.3931 \\
1.6217 \\
1.8575\end{array}$ & $\begin{array}{l}.0018 \\
.0018 \\
.0022 \\
.0025 \\
.0027\end{array}$ & $\begin{array}{l}1.0352 \\
1.1602 \\
1.3953 \\
1.6242 \\
1.8602\end{array}$ & $\begin{array}{l}\text { 1. } 1250 \\
\text { 1. } 2500 \\
1.5000 \\
1.7500 \\
\text { 2. } 0000\end{array}$ & $\begin{array}{l}1.0322 \\
1.1572 \\
1.3917 \\
1.6201 \\
1.8557\end{array}$ & $\begin{array}{l}0 \\
0 \\
0 \\
0 \\
0\end{array}$ & $\begin{array}{l}16 \\
16 \\
16 \\
16 \\
15\end{array}$ \\
\hline $\begin{array}{l}21 / 4 \ldots \\
21 / 2 \\
23 / 4 \\
3\end{array}$ & $\begin{array}{l}41 / 2 \\
4 \\
4 \\
4\end{array}$ & $\begin{array}{l}2.2578 \\
2.5060 \\
2.7590 \\
3.0090\end{array}$ & $\begin{array}{l}.1764 \\
.1985 \\
.1985 \\
.1985\end{array}$ & $\begin{array}{l}2.1075 \\
2.3396 \\
2.5896 \\
2.8396\end{array}$ & $\begin{array}{l}.0027 \\
.0030 \\
.0030 \\
.0030\end{array}$ & $\begin{array}{l}2.1102 \\
2.3426 \\
2.5926 \\
2.8426\end{array}$ & $\begin{array}{l}2.2500 \\
2.5000 \\
2.7500 \\
3.0000\end{array}$ & $\begin{array}{l}2.1057 \\
2.3376 \\
2.5876 \\
2.8376\end{array}$ & $\begin{array}{l}0 \\
0 \\
0 \\
0\end{array}$ & $\begin{array}{l}15 \\
15 \\
15 \\
15\end{array}$ \\
\hline
\end{tabular}

NotE. -Maximum lead error $= \pm 0.0005$ inch in 1 inch of thread. 
TABLE 24.-Dimensions and tolerances for class $Y$ ground taps, national finethread series

\begin{tabular}{|c|c|c|c|c|c|c|c|c|c|}
\hline \multirow[b]{2}{*}{ Sizes } & \multirow[b]{2}{*}{$\begin{array}{l}\text { Threads } \\
\text { perinch }\end{array}$} & \multicolumn{2}{|c|}{ Major diameter } & \multicolumn{3}{|c|}{ Pitch diameter } & \multicolumn{2}{|c|}{ Basic diameter } & \multirow[b]{2}{*}{$\begin{array}{l}\text { Toler- } \\
\text { ance on } \\
\text { half angle } \\
\text { of thread }\end{array}$} \\
\hline & & $\begin{array}{l}\text { Mini- } \\
\text { mum }\end{array}$ & $\begin{array}{c}\text { Maxi- } \\
\text { mumim = } \\
\text { measured } \\
\text { pitched } \\
\text { diameter } \\
\text { plus }\end{array}$ & $\begin{array}{l}\text { Mini- } \\
\text { mum }\end{array}$ & $\begin{array}{l}\text { Toler- } \\
\text { ance }\end{array}$ & $\begin{array}{l}\text { Maxi- } \\
\text { mum }\end{array}$ & Major & Pitch & \\
\hline 1 & 2 & 3 & 1 & 5 & 6 & 7 & 8 & 9 & 10 \\
\hline & $\begin{array}{l}28 \\
24 \\
24 \\
20 \\
20\end{array}$ & $\begin{array}{r}\text { Inches } \\
0.2525 \\
.3150 \\
.3775 \\
.4405 \\
.5030\end{array}$ & $\begin{array}{c}\text { Inch } \\
0.0284 \\
.0331 \\
.0331 \\
.0397 \\
.0397\end{array}$ & $\begin{array}{c}\text { Inches } \\
0.2273 \\
.2859 \\
.3484 \\
.4055 \\
.4680\end{array}$ & $\begin{array}{l}\text { Inch } \\
0.0010 \\
.0010 \\
.0010 \\
.0010 \\
.0010\end{array}$ & $\begin{array}{r}\text { Inches } \\
0.2283 \\
.2869 \\
.3494 \\
.4065 \\
.4690\end{array}$ & $\begin{array}{c}\text { Inches } \\
0.2500 \\
.3125 \\
.3750 \\
.4375 \\
.5000\end{array}$ & $\begin{array}{c}\text { Inches } \\
0.2268 \\
.2854 \\
.3479 \\
.4050 \\
.4675\end{array}$ & $\begin{array}{cc}\text { Deg. } & \text { Min. } \\
0 & 24 \\
0 & 22 \\
0 & 22 \\
0 & 20 \\
0 & 20\end{array}$ \\
\hline & $\begin{array}{l}18 \\
18 \\
16 \\
14 \\
14\end{array}$ & $\begin{array}{r}.5655 \\
.6280 \\
.7536 \\
.8787 \\
1.0037\end{array}$ & $\begin{array}{l}.0441 \\
.0441 \\
.0496 \\
.0567 \\
.0567\end{array}$ & $\begin{array}{l}.5269 \\
.5894 \\
.7100 \\
.8293 \\
.9543\end{array}$ & $\begin{array}{l}.0010 \\
.0010 \\
.00011 \\
.0012\end{array}$ & $\begin{array}{l}.5279 \\
.5904 \\
.7111 \\
.8305 \\
.9555\end{array}$ & $\begin{array}{r}.5625 \\
.6250 \\
.7500 \\
.8750 \\
1.0000\end{array}$ & $\begin{array}{l}.5264 \\
.5889 \\
.7094 \\
.8286 \\
.9536\end{array}$ & $\begin{array}{ll}0 & 20 \\
0 & 20 \\
0 & 19 \\
0 & 19 \\
0 & 19\end{array}$ \\
\hline & $\begin{array}{l}12 \\
12 \\
12\end{array}$ & $\begin{array}{l}1.1298 \\
1.2548 \\
1.5048\end{array}$ & $\begin{array}{l}.0662 \\
.0662 \\
.0662\end{array}$ & $\begin{array}{l}1.0717 \\
1.1967 \\
1.4467\end{array}$ & $\begin{array}{l}.0014 \\
.0014 \\
.0014\end{array}$ & $\begin{array}{l}1.0731 \\
1.1981 \\
1.4481\end{array}$ & $\begin{array}{l}\text { 1. } 1250 \\
\text { 1. } 2500 \\
\text { 1. } 5000\end{array}$ & $\begin{array}{l}\text { 1. } 0709 \\
\text { 1. } 1959 \\
1.4459\end{array}$ & $\begin{array}{ll}0 & 18 \\
0 & 18 \\
0 & 18\end{array}$ \\
\hline
\end{tabular}

Nate. - Maximum lead error $= \pm 0.0005$ inch in 1 inch of thread.

\section{(c) TAP-DRILL SIZES}

\section{Classes 1 to 4 , inclusive}

The essential requirement of a tap drill is that the hole produced by it shall be such that, when tapped with a screw thread, the minor diameter of the tapped hole shall be within the specified limits shown in Tables 7 to 14 , inclusive, herein.

If the drill is too large, the minor diameter of the tapped hole will also be too large and the thread in the nut will be too shallow; that is, too small a percentage of a full thread. As an extreme case the threads in the nut will engage only the tops of the threads on a bolt of correct size, and under stress the nut member will not sustain the proper load.

If, on the other hand, the tap drill is too small, the tap will be forced to cut a thread of full depth, and in the extreme case to act as a reamer also. This will result in excessive power consumption and tap breakage, and will also make the minor diameter of the tapped hole dependent upon the minor diameter of the tap. This is undesirable, since the minor diameter of the tap is not, in general, held to the same close limits as the other tap elements, and as a result the minor diameter of a hole tapped under these conditions may be in error even though the tap is otherwise correct.

It is a well-known fact that the size of the hole produced by a tap drill depends to some extent upon the method of grinding the drill, the material drilled, the lubricant used, and the speed and feed of 
operation. This being true, it is apparent that fixing the diameter of the tap drill does not completely fix the diameter of the drilled hole. The most that can be accomplished is to fix the drill diameters between certain limits and to depend upon correct grinding, lubrication, and operation to keep the diameter of the holes within prescribed limits.

In establishing tap-drill sizes, the starting point is the mean between the maximum and minimum minor diameter of the tapped hole. This permits a variation of the minor diameter, both above and below the ideal tap-drill size, equal to half the tolerance on minor diameter. Thus the tap drill may cut oversize by this amount by reason of incorrect grinding, lubrication, or speration, or on account of differences in the materials tapped; and it may be allowed to wear undersize until the tapped hole reaches the lower limit on minor diameter.

It should be noted that the minor diameters of the tapped holes are the same for classes 1 to 4 , inclusive. This condition results from the requirement on page 18 with reference to clearance on minor diameter:

4. Clearance on Minor Diameter.-A clearance shall be provided at the minor diameter of the nut by removing from the crest of the basic thread form an amount equal to one-sixth to one-fourth of the basic thread depth.

The minor diameter of the tapped hole is therefore greater than the basic minor diameter by one-third to one-half the basic thread depth, and the tolerance on the minor diameter is one-sixth the basic depth.

Minimum minor diameter of tapped hole $=$ basic minor diameter $+\frac{h}{3}$

Maximum minor diameter of tapped hole $=$ basic minor diameter $+\frac{h}{2}$ in which

$$
\begin{aligned}
& h=\text { basic thread depth }=0.649519 p \\
& p=\text { pitch }=\frac{1}{n} \\
& n=\text { number of threads per inch. }
\end{aligned}
$$

There are given in Tables 25 and 26 the limiting dimensions of the minor diameter of the threaded hole, as established by the commission, together with the tap-drill sizes recommended. There are also given the diameters of all the drills regularly carried in stock that fall between the limiting dimensions of the minor diameter of the threaded hole. Accordingly, drills of these diameters should produce holes which result in a thread depth of 75 to $831 / 3$ per cent of the basic depth of thread.

The commission recommends to manufacturers of tap drills the making up of complete sets of drills of the sizes required for both the "national coarse" and "national fine thread series." The user 
will then be able to select at once the correct drill for any national standard thread without hunting 'through the several sets of numbered, lettered, fractional, and metric sizes ordinarily carried in stock. The drills should be marked in accordance with the instructions on page 17. For example, $1^{\prime \prime}-8-N C$ would be used to designate the drill to be used for a 1-inch, eight threads per inch, national coarse thread.

TABLE 25.-Sizes of tap drills, national coarse-thread series

\begin{tabular}{|c|c|c|c|c|c|c|c|c|}
\hline \multirow[t]{2}{*}{ Size of thread } & \multirow{2}{*}{$\begin{array}{l}\text { Threads } \\
\text { per inch }\end{array}$} & \multicolumn{3}{|c|}{ Minor diameter of nut } & \multirow{2}{*}{$\begin{array}{l}\text { Recom- } \\
\text { mended } \\
\text { tap drill } \\
\text { diameter }\end{array}$} & \multicolumn{2}{|c|}{$\begin{array}{l}\text { Present stock tap drills } \\
\text { leaving between } 831 / 3 \\
\text { and } 75 \text { per cent of basic } \\
\text { thread depth }\end{array}$} & \multirow{2}{*}{$\begin{array}{l}\text { Per cent } \\
\text { of depth } \\
\text { of basic } \\
\text { thread }\end{array}$} \\
\hline & & Basic & $\begin{array}{l}\text { Maxi- } \\
\text { mum }\end{array}$ & $\begin{array}{l}\text { Mini- } \\
\text { mum }\end{array}$ & & Nominal size & Diameter & \\
\hline 1 & 2 & 3 & 4 & 5 & 6 & $\gamma$ & 8 & 9 \\
\hline $1 \ldots$ & 64 & $\begin{array}{c}\text { Inches } \\
0.0527\end{array}$ & $\begin{array}{c}\text { Inches } \\
0.0578\end{array}$ & $\begin{array}{l}\text { Irches } \\
0.0561\end{array}$ & $\begin{array}{l}\text { Inches } \\
10.0575\end{array}$ & & Inches & 76 \\
\hline $2 \ldots$ & 56 & .0628 & .0686 & .0667 & 1.0682 & $\begin{array}{l}1.7 \mathrm{~mm} \text {.- } \\
\text { No. } 51 .-\end{array}$ & $\begin{array}{r}0.0669 \\
.0670\end{array}$ & $\begin{array}{l}82 \\
82\end{array}$ \\
\hline $3 \ldots$ & 48 & .0719 & .0787 & .0764 & 0781 & $\begin{array}{l}\frac{5}{66} \text { in } \\
\text { No. } 47=- \\
2 \mathrm{~mm}\end{array}$ & $\begin{array}{l}.0781 \\
.0785 \\
0788\end{array}$ & $\begin{array}{l}77 \\
76 \\
-5\end{array}$ \\
\hline $4 \ldots$ & 40 & .0795 & .0876 & .0849 & .0866 & $\begin{array}{l}\text { No. } 44 \text { - } \\
2.2 \mathrm{~mm} .\end{array}$ & $\begin{array}{l}.0860 \\
.0866\end{array}$ & $\begin{array}{l}80 \\
78\end{array}$ \\
\hline $5 \ldots$ & 40 & .0925 & .1006 & .0979 & $\begin{array}{l}.0995 \\
.063\end{array}$ & $\begin{array}{l}2.5 \mathrm{~mm} \\
\text { No. } 39\end{array}$ & $\begin{array}{l}.0984 \\
.0995 \\
1063\end{array}$ & $\begin{array}{l}80 \\
78 \\
78\end{array}$ \\
\hline $\begin{array}{l}6 \ldots \\
8\end{array}$ & 32 & .0974 & .1056 & .1042 & & No. $36_{--}$ & .1065 & 78 \\
\hline $0_{-}$ & 32 & .1234 & .1336 & .1302 & 1324 & $3.7 \mathrm{~mm}$ & .1457 & $\begin{array}{l}78 \\
82\end{array}$ \\
\hline $10 \ldots$ & 24 & .1359 & .1494 & .1449 & .1476 & $\begin{array}{l}\text { No. } 26 \\
3.75 \mathrm{~mm} \\
1 .- \\
6 \text { in }\end{array}$ & $\begin{array}{l}.1470 \\
.1476 \\
.1719\end{array}$ & $\begin{array}{l}79 \\
78 \\
82\end{array}$ \\
\hline $12-$. & 24 & .1619 & .1754 & .1709 & .1732 & $\begin{array}{l}\text { No. } 17 \\
\text { No. } 9 \text { m }\end{array}$ & $\begin{array}{l}.1730 \\
.1732 \\
.1960\end{array}$ & $\begin{array}{l}79 \\
79 \\
83\end{array}$ \\
\hline $1 / 4-$ & 20 & .1850 & .2013 & .1959 & .1990 & $\begin{array}{l}\text { No. } 8 \\
5.1 \mathrm{~mm} \\
\text { No. } 7\end{array}$ & $\begin{array}{l}.1990 \\
.2008 \\
.2010\end{array}$ & $\begin{array}{l}78 \\
78 \\
76 \\
75\end{array}$ \\
\hline$\frac{1}{16--}$ & 18 & .2403 & .2584 & .2524 & .2559 & $6.5 \mathrm{~mm}_{---}$ & .2539 & 78 \\
\hline $3 / 8$ & 16 & .2938 & .3141 & .3073 & .3110 & $7.9 \mathrm{~mm}$ & .3110 & $\begin{array}{l}79 \\
77\end{array}$ \\
\hline$\frac{7}{16}--$ & 14 & .3447 & $.36 \pi 9$ & .3602 & .3643 & $9.25 \mathrm{~mm}$ & .3642 & 79 \\
\hline $1 / 2-$ & 13 & .4001 & .4251 & .4167 & .4219 & $\frac{27}{6}$ in $\ldots$ & .4219 & 78 \\
\hline$\frac{8}{18}-1$ & 12 & .4542 & .4813 & .4723 & 1.4776 & $12 \mathrm{~mm}$ & .4824 & $\begin{array}{l}83 \\
78\end{array}$ \\
\hline $3 / 8-$ & 11 & .5069 & .5364 & .5266 & .5315 & $\begin{array}{l}\frac{1}{31} \text { in } \\
13.5 \mathrm{~mm}\end{array}$ & $\begin{array}{l}.5312 \\
.5315\end{array}$ & $\begin{array}{l}80 \\
79\end{array}$ \\
\hline $3 / 4$ & 10 & .6201 & .6526 & .6417 & 6480 & $16.5 \mathrm{~mm}$ & .6496 & 77 \\
\hline $7 / 8$ & 9 & .7307 & .7667 & .7547 & & 6 & .7656 & 80 \\
\hline 1. & 8 & .8376 & .8782 & .8647 & $\left\{\begin{array}{r}1.8723 \\
-0.80\end{array}\right.$ & $7 / 8$ in & .8750 & $\begin{array}{l}82 \\
79 \\
77\end{array}$ \\
\hline $11 / 8$ & 7 & .9394 & .9858 & .9704 & & $\begin{array}{l}25 \mathrm{~mm} \\
\frac{85}{68}\end{array}$ & $\begin{array}{r}.9842 \\
.9844 \\
\end{array}$ & $\begin{array}{l}76 \\
76\end{array}$ \\
\hline $11 / 4$ & 7 & 1.0644 & 1. 1108 & 1.0954 & 1. 1024 & $\begin{array}{l}28 \mathrm{~mm}_{-} \\
1 \frac{7}{6 \mathrm{k}} \mathrm{in}\end{array}$ & $\begin{array}{l}1.1024 \\
1.1094\end{array}$ & 76 \\
\hline $11 / 2$ & 6 & 1.2835 & 1. 3376 & 1.3196 & 1.3281 & $39 \mathrm{~mm}$ & $\begin{array}{l}\text { 1. } 3281 \\
1.5354\end{array}$ & $\begin{array}{l}79 \\
83 \\
79\end{array}$ \\
\hline & 5 & 1.4902 & 1.5551 & 1.5335 & $\left\{\begin{array}{l}1.3403 \\
-1.0 .\end{array}\right.$ & $\mid \begin{array}{l}1,85 \\
39.5 \mathrm{~mm}\end{array}$ & $\begin{array}{l}1.5469 \\
1.5551\end{array}$ & $\begin{array}{l}78 \\
75\end{array}$ \\
\hline
\end{tabular}

1 Not a stock size. 
TABLE 25.-Sizes of tap drills, national coarse-thread series-Continued

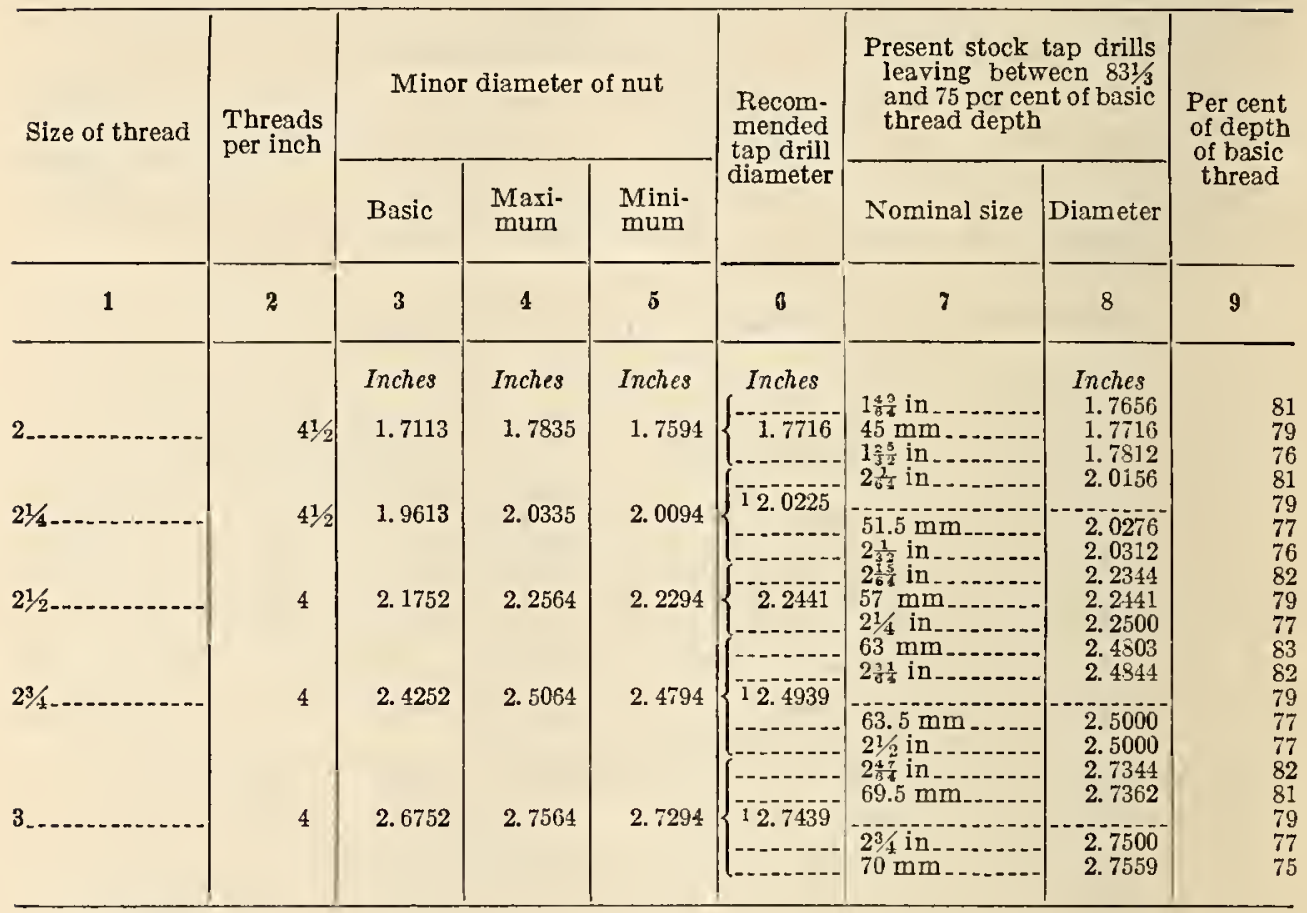

1 Not a stock size.

TABLE 26.-Sizes of tap drills, national fine-thread series

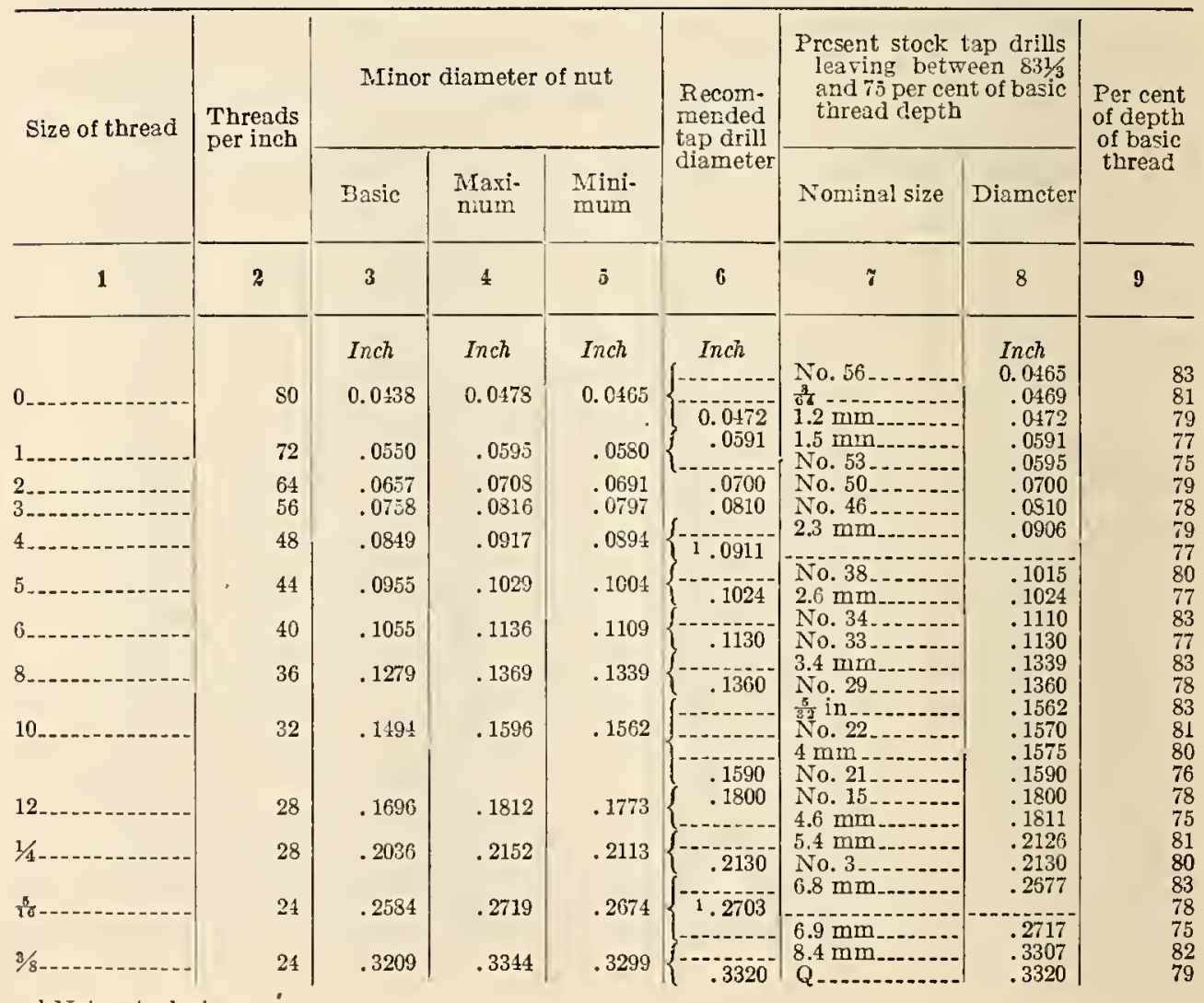

1 Not a stock size. 
1924 REPORT

TABLE 26.-Sizes of tap drills, national fine-thread series-Continued

\begin{tabular}{|c|c|c|c|c|c|c|c|c|}
\hline \multirow{2}{*}{ Size of thread } & \multirow{2}{*}{$\begin{array}{l}\text { Threads } \\
\text { per inch }\end{array}$} & \multicolumn{3}{|c|}{ Minor diameter of nut } & \multirow{2}{*}{$\begin{array}{l}\text { Recom- } \\
\text { mended } \\
\text { tap drill } \\
\text { diameter }\end{array}$} & \multicolumn{2}{|c|}{$\begin{array}{l}\text { Present stock tap drills } \\
\text { leaving between } 831 / 3 \\
\text { and } 75 \text { per cent of basic } \\
\text { thread depth }\end{array}$} & \multirow{2}{*}{$\begin{array}{l}\text { Per cent } \\
\text { of depth } \\
\text { of basic } \\
\text { thread }\end{array}$} \\
\hline & & Basic & $\begin{array}{l}\text { Maxi- } \\
\text { mum }\end{array}$ & $\begin{array}{l}\text { Mini- } \\
\text { mum }\end{array}$ & & Nominal size & Diameter & \\
\hline \multirow[t]{5}{*}{1} & 2 & 3 & 4 & 5 & 6 & 7 & 8 & 9 \\
\hline & & Inches & Inches & Inches & Inches & $9.75 \mathrm{~mm}$ & $\begin{array}{l}\text { Inches } \\
0.3839\end{array}$ & \multirow{10}{*}{$\begin{array}{l}82 \\
80 \\
79 \\
78 \\
78 \\
78 \\
75 \\
75 \\
75 \\
78 \\
81 \\
78 \\
75 \\
78 \\
78 \\
76\end{array}$} \\
\hline & 20 & 0.3725 & 0.3888 & 0.3834 & 0.3860 & $9.8 \mathrm{~mm} \ldots$ & $\begin{array}{l}.3858 \\
.3860\end{array}$ & \\
\hline & $\begin{array}{l}20 \\
18\end{array}$ & $\begin{array}{r}.4350 \\
.4903\end{array}$ & $\begin{array}{r}.4513 \\
.5084\end{array}$ & $\begin{array}{l}.4459 \\
.5024\end{array}$ & $\begin{array}{r}1.4492 \\
1.5062 \\
1.5687\end{array}$ & 1 $1-1.0$. & - & \\
\hline & 18 & .5528 & .5709 & .5649 & 1.5687 & $14.5 \mathrm{~mm}$ & .5709 & \\
\hline $3 / 4--$ & 16 & .6688 & .6891 & .6823 & .6875 & $\begin{array}{l}\frac{1}{12} \text { in } \\
17.5 \mathrm{~mm}\end{array}$ & $\begin{array}{l}.6875 \\
.6890\end{array}$ & \\
\hline $7 / 8-$. & 14 & .7822 & .8054 & .7977 & i. 8024 & & & \\
\hline $1 \ldots$ & 14 & .9072 & .9304 & .9227 & $\{-9.9274$ & $23.5 \mathrm{~mm}$ & .9252 & \\
\hline $11 / 8$ & 12 & 1. 0167 & 1. 0438 & 1. 0348 & 1.0101 & $26.5 \mathrm{~mm}$ & 1. 0433 & \\
\hline $11 / 4--$ & 12 & 1.1417 & 1. 1688 & 1.1598 & $\{1,1651$ & $29.5 \mathrm{~m}$ & 1. 1614 & \\
\hline $11 / 2-$ & 12 & 1.3917 & 1. 4188 & 1. 4098 & $\left\{\begin{array}{l}1.4103 \\
-\ldots \ldots\end{array}\right.$ & $36 \mathrm{~mm}$ & 1.4173 & \\
\hline
\end{tabular}

1 Not a stock size.

\section{GAGES}

The art of measuring screw threads has developed very rapidly during the past few years. This development still continues, so that it would be inadvisable to attempt to specify any definite method as standard for this purpose. The object is to establish the fundamentals of this subject, and to point out practices now successfully used.

\section{(a) FUNDAMENTALS}

1. Oвject of Gaging.-The final results sought by gaging are to secure interchangeability; that is, the assembly of mating parts without selection or fitting of one part to another, and to insure that the product conforms to the specified dimensions within the limits of variation establishing the closest and loosest conditions of fit permissible in any given case, as provided for in the foregoing specifications. This requires the use of gages representing the limit of maximum metal which must assemble with the product, known as "go" gages, and gages representing the limit of minimum metal which must not assemble with the product, known as "not go" gages.

Gaging should be as much employed to prevent unsatisfactory parts from being produced as to sort out the correct from the incorrect parts.

2. Purpose of "Go" and "Not go" Gages.-The "go" gage, which is the gage representing the maximum limit of the internal member or the minimum limit of the external member, controls the allowance between mating surfaces, and also controls interchange- 
ability. "Go" gages control the maximum tightness in the fit of mating parts. Parts which are acceptable to proper "go" gages will always interchange. Successful interchangeable manufacturing has been carried on for many years with the use of "go" gages only.

The "not go" gage, which is the gage representing the minimum limit of the internal member or the maximum limit of the external member, controls the extent of the tolerance in one direction. "Not go" gages limit the amount of looseness between mating" parts, and thus control, in large measure, the proper functioning of mating parts.

3. Gage Classmiration.--The limiting dimensions of the threaded parts to be produccd must be represented in: (a) Gages used in checking the product as it is machined, known as "working gages"; (b) gages for use in the acceptancc of the product, known as "inspcction gages"; and (c) gages used to determine the accuracy of the two preceding classes of gages, known as "mastcr gages."

4. Gages Used to Measure the Product.-The gages used to check the product may be divided into two general types: "Mechanical" and "optical." Both types, however, arc controlled by the master gages. Most of the product accepted by one type of gaging with a correct gage will be accepted by the other. It should be pointcd out, however, that those parts which are ncar either rejection point may be accepted by one system and rejected by the other.

(a) Mechanical gages. - Mechanical gages ordinarily comprise the inspection and working gages as abovc dcfined, and these two classes are generally of the same design. The dimensions of inspection gages are such that they represent very nearly the extreme limits of the part. It is recommended that, when successive inspections are required, the working gages, either by design or selection, be of such dimensions that they are inside the limits of the gages used in succeeding inspections.

(b) Optical gages.-When gages of the optical type are employed, the elements of wear and "fecl" are not involved, bence no difference in size between inspection and working gages is necessary, but is desirable.

5. Gages For Reference.-(a) Master gage.-The "master gage" is a plug-thread gage which represents the physical dimensions of the nominal or basic size of the part. A master gage shall be accompanied by a record of its measurement. In case of question, the deviations of this gage from the basic size shall be ascertained by the Bureau of Standards at Washington, D. C.

(b) Setting gage (check gage).-A setting gage is a plug-thread gage to which adjustable ring-thread gagcs and other thread comparators are adjusted for size.

\footnotetext{
${ }^{7}$ Recommendations as to the design and construction of mechanical gages are given in Appendix 4.
} 
6. Direction of Tolerances on Gages.-The extreme sizes for limit gages shall never exceed either extreme limit of the part being produced. All variations in the gages, whatever their cause or purpose, shall bring these gages within these extreme limits. Thus, a gage which represents a minimum limit may be larger, but never smaller, than the minimum size specified for the part, while the gage which represents a maximum limit may be smaller, but never larger, than the maximum size specinied for the part.

7. Temperature at Which Gages Shall be Standard.-Gages and product shall have their correct nominal dimensions at a temperature of $68^{\circ} \mathrm{F}$. $\left(20^{\circ} \mathrm{C}\right.$.).

As gages and products are ordinarily checked at room temperature, whatever it may happen to be, it is desirable that the thermal coefficient of expansion of gages be the same as that of the product on which they are used. Inasmuch as the majority of threaded products consist of iron and steel, and as screw-thread gages are ordinarily made of hardened steel, because of its high wear-resisting qualities, this condition is ordinarily fulfilled without giving it special attention.

8. Measuring Pressure for Three-Wire Measurements.-A contact pressure of 2 to 3 pounds shall be used in measuring the pitch diameter of screw-thread gages by means of wires.

\section{(b) GAGING PRACTICES AND TYPES OF GAGES}

The production of accurate parts is primarily a matter of constant vigilance and of training of workmen. The smaller the tolerances which are to be maintained, the more complete the inspection or gaging system must be. In order to secure satisiactory results, the manufacturing tools provided must be sufficiently accurate, and the manufacturing methods must be sufficiently reliable, to produce the required results. After tools and methods of proved reliability are provided, it is necessary to watch the wear on the tools or changes in their set-up to insure that the required conditions are maintained. This is accomplished by periodical tests of the tools, by periodical gaging of the product, and sometimes by both.

There is a broad, general principle in regard to limit gages which should be kept in mind; a "go" gage should check simultaneously as many elements as possible, a "not go" gage, to be effective, can check but one element. By effective inspection is meant assurance that specified requirements in regard to size are not exceeded. A "not go" thread gage made to check the pitch diameter only is usually sufficient for practical purposes.

A screw thread comprises several elements: First, the major diameter; second, the pitch diameter; third, the minor diameter; fourth, the angle of the thread; and fifth, the lead. The most difficult element of a screw thread to gage is the lead. Lead-testing derices for checking tools and gages are available, but, in general, their opera- 
tion is too slow for use as production inspection equipment. In addition, the lead is the most important element of a screw thread as regards the nature of the contact between the surfaces of the mating parts. Furthermore, the result of an error in lead is almost double that of an equal error in diameter as regards interchangeability. For exacting threaded work, if the method of inspection of the product does not effectively detect lead errors, the tools used must be carefully inspected for lead. In order to reduce the possibilities of disagreement to a minimum, the manufacturer should strive to produce parts well within the specified limits rather than close to the limiting sizes.

1. Thread Micnometers.-Thread micrometers are extensively used to measure the pitch diameter of taps and screws. Thread micrometers should be calibrated periodically against a master gage, to avoid errors due to vear on the anvils of the instrument. As thread micrometers give no indication of lead and angle errors, the results of tests made with thread micrometers alone can not be taken as conclusive.

2. Thread Snap Gages.-Thread snap gages are generally adjustable and have contact points consisting of cone-pointed anvils, wedgeshaped prisms with rounded edges, serrated or grooved plates, or grooved or threaded cylinders adjustably mounted and suitably spaced in a U-shaped frame. These gages are used to some extent in gaging external threads, and have the advantages that work may be inspected with great rapidity by the single motion of passing it between the anvils of the gage, and given a visual examination for clearance as well as a tactile inspection. The positions of the anvils are set to a setting gage, and the anvils are then clamped in position and sealed.

The usual form of cone-pointed snap gage has a single point on each side of the frame, and is an effective "not go" gage. It does not, however, fully meet the requirements for a "go" gage, as it does not check the lead, and, therefore, must be supplemented with some type of indicating gage to check the lead when used for checking pitch diameter, angle, and thread form. Also, as it checks only a single diameter at a time, the "go" snap gage must be tried at a series of points to determine whether the maximum diameter of an external thread is within the tolerance. When provided with three contact points, two on one side spaced an integral number of threads apart and one on the other, such a gage checks the lead for progressive, but not always for local or periodic lead errors, and, thus, it more nearly fulfills the requirements for a "go" thread gage.

Thread snap gages having multiple toothed contact points; that is, toothed blades, serrated or grooved plates, or grooved or threaded cylinders are made in a variety of forms, either as separate or combined "go" and "not go" gages. The fit of a screw in such a gage is affected by variations in pitch diameter, lead, and angle of the screw, 
and the gage accordingly may be used as a "go" gage. Such gages have been found suitable only for the less accurate classes of work, such as the loose and free fit classes.

Thread micrometers and thread snap gages are extensively used for testing the product as it is produced. As these instruments do not test all elements of the screw thread, a "go" gage should always be used as a supplementary test. Thread micrometers are very effective means of checking against the change in set-up due to wear on tools, etc.

3. Ring Thread Gages.-Ring thread gages are extensively used to measure the threads on screws. These are usually adjustable to suitable setting gages. When the product is to be within specified limits, "go" and "not go" gages are required. The use of such gages gives some information as to lead and angle errors as well as pitch diameter errors.

4. Thread Comparators.-A recent development in the art of measuring threaded parts is the optical thread comparator, which embodies the principle of gaging in an optical projection system. In addition to giving a rapid indication of whether the elements of the screw thread lie within the limiting dimensions specified, such instruments furnish more detailed information as to the errors in screw threads than is usually obtained by means of mechanical gages, particularly as to irregularities in thread form, lead and diameter. These instruments can be adapted to measure taps and other threading tools.

The available forms of projection comparators differ somewhat in design and principle, but each consists primarily of a source of parallel light, such as an electric arc or concentrated filament lamp with condensing lens system, a projection lens system, a screen upon which the magnified shadow image of the work is projected, and a device for holding the work in position in front of the projection lenses. The screen consists of a tolerance chart on which two outlines of the correct thread form at the magnification used are spaced one above the other a distance equal to the tolerance multiplied by the magnification. The chart and gage holder are adjusted to position by projecting the shadow image of a setting gage and adjusting to bring the outline of the shadow image and certain lines of the chart into coincidence, after which the system may be used as a gaging device.

5. Indicating Gages.-An indicating thread gage has movable contact points, which are set to a setting gage, and is intended to give an exact indication of the variations of the dimensions of a screw thread within the specified limits, rather than to show merely that the thread is within, or outside of, the specified limits, as is the case with limit gages. In such gages, the movable contact points actuate a multiplying lever system, or other means for magnifying their motion, 
and the amount of the motion is registered on a graduated dial or scale. Indieating gages are made aceording to a variety of designs, some to indieate progressive lead error only, some to indicate pitch diameter only, some to indieate both separately but on the same gage, others to indieate the major and minor diameters as well, and still others to indieate the apparent size. They have been applied almost exelusively to external threads. Those whieh indieate the apparent size may be considered as most nearly fulfilling the requirements of a gaging system. However, those indieating lead crrors are very useful in eontrolling lead errors in threading tools and serew-thread produets. Also eertain types can be used to indieate the variation in roundness on piteh or major diameters.

6. Plug Thread Gages. - At the present time the most praetieal means of gaging threaded holes or nuts is by the use of plug thread gages. When the produet is to be within speeified limits, "go" and "not go" gages are required. The use of sueh gages gives some information as to lead and angle errors as well as piteh diameter errors. A eorreet "go" plug gage will rejeet any parts whieh exceed the minimum dimensions speeified.

One praetiee of inspeeting tapped holes is to first inspect the tap, and then to test the tapped holes periodically with "go" and "not go" gages. The tap ean be watched for wear by testing the tapped holes with a "go" thread gage. One widely used practice consists of using a "go" plug thread gage, and a "not go" plain plug gage for the minor diameter.

One practiee of inspeeting taps is to measure the several elements, sueh as piteh diameter, angle, and lead. Another praetice eonsists of tapping a hole with eaeh tap before it is issued from the tool erib and testing these tapped holes with "go" and "not go" plug thread gages.

Sometimes the tap is tested after it is returned to the tool erib. If it is eorreet, it is replaeed in its proper eompartment. If it has worn below the limits, it is discarded and work produced by it is eheeked and eorreeted when necessary.

7. Plain Gages.- "Go" and "not go" plain eylindrical plug gages are used for inspeeting the minor diameter of the tapped hole. Plain ring or snap gages are used for inspeeting the major diameter of the serew. When used, it is recommended that the "go" inspeetion gage be a ring gage and the "not go" inspeetion gage be a snap gage. The working gages may be a combined "go" and "not go" snap gage.

8. Testing of Gages.-Gages should be tested periodieally for wear and to insure that the gages are properly distributed. When suceessive inspeetions in the same plant are involved, it is good practiee to inspeet all gages of the same nominal size against each 
other periodically, and to distribute these gages so that the earlier inspections are made with those which are the greatest amount inside of the component limits, while the later inspections are made with those gages closest in size to the component limits.

The original testing of a thread gage should include measurements for diameters, angle, and lead. If these elements test satisfactorily, the later inspection need be measurements of pitch diameter only. ${ }^{8}$

\section{(c) SPECIFICATIONS FOR GAGES}

The following specifications are for the purpose of establishing definite limits for thread gages rather than for the purpose of specifying the gages required for the various inspection operations.

1. Classification of Gages, and Gage Tolerances.-Screw thread gages are classified according to accuracy into classes $\mathrm{X}, \mathrm{Y}$, and $\mathrm{Z}$, the class $\mathrm{X}$ being the most accurate. The tolerance limits on classes $Y$ and $Z$ "go" gages are placed inside of the extreme product limits to provide allowance for wear of the gages. The tolerances on all "not go" gages, however, are applied from the extreme product limit as the starting point, as no allowance for wear is necessary. The selection of gages from among these classes for use in the inspection of threaded product depends entirely upon the specifications for the product. For cxample, in the production of parts to class 4 , close fit specifications, class $\mathrm{X}$ gages may be required for all purposes. On the other hand, for parts made to class 1 , loose fit specifications, class $\mathrm{Z}$ gages may be sufficiently accurate for all purposes.

(a) Master gages.-No fixed tolerances are specified for master gages. These should be made to the basic size as accurately as possible and be within the tolerances specified for class $\mathrm{X}$ gages. The variations from basic size shall be plus. Each master gage shall be marked with an identification number or symbol, and be accompanied by a record of its measurement on major diameter, pitch diameter, lead, and angle. In case of question, the deviations of such gages from the exact standard shall be ascertained by the Bureau of Standards at Washington, D. C.

(b) Class $X$ gages.-Class $\mathrm{X}$ gages should be suitable for inspection and setting gages for all classes of fits. They may also be necessary for working gages for class 4 fits. The tolerances on these gages shall be as given in Table 28. In all cases the tolerances shall be such that the gage does not fall outside of the component tolerances. For example, if a plug thread gage is used as the "go" gage for checking a tapped hole, it can be larger, but not smaller than the minimum size specified. On the other hand, if a plug thread gage is used as the "go" setting plug for ring thread gages or for optical or other comparators, it can be smaller, but never larger than the maximum size of the screw.

Methods of measuring pitch diameter of screw thread gages are described in Appendix 2. 
Class $\mathrm{X}$ tolerances, as given in Table 28, are specified for all "not go" gages.

(c) Class $Y$ gages.-Class $Y$ gages should be suitable for inspection gages for classes 1, 2, and 3 fits. They may also be desired as working gages for classes 2 and 3 fits. The tolerances on these gages shall be as given in Table 29.

(d) Class $Z$ gages.-Class Z gages should be suitable for working gages for class 1 , loose fit. The tolerances on these gages shall be as given in Table 30.

(e) Wear on gages.- "Go" gages may be permitted to wear to the extreme product limits. It is desirable, however, that working and inspection gages be so selected that the dimensions of the working gages are inside of the limiting dimensions represented by the inspection gages, in order that all parts passed by the working gage will be accepted by the inspection gage.

As to wear on "not go" gages, it is purely a question of economy as to when the "not go" gage should be discarded. Continued use reduces the available working tolerance on the product, and the resulting loss must be balanced against the cost of a new gage.

(f) Tolerance on lead.-The tolerances on lead given in Tables 28 to 30 , inclusive, are specified as an allowable variation between any two threads not farther apart than the length of engagement of the assembled threaded product. When this length of engagement is equal to the diameter, the permissible progressive lead errors per inch may be determined by diriding these lead tolerances by the corresponding diameters.

(g) Tolerances on angle of thread.-The tolerances on angle of thread, as specified in Tables 28 to 30 , inclusive, for the various pitches, are tolerances on one-half of the included angle. This insures that the bisector of the included angle will be perpendicular to the axis of the thread within proper limits. The equivalent deviation from the true thread form caused by such irregularities as convex or concave sides of thread, rounded crests, or slight projections on the thread form, should not exceed the tolerances permitted on angle of thread.

2. Tolerayces for Plain Gages.-For plain plug gages, plain ring gages, and plain snap gages required for measuring diameters of screw-thread work, the gage tolerances specified in Table 31 should be used. Attention is directed to the fact that the tolerances on thread diameters vary in accordance with the number of threads per inch. In manufacturing a plain plug, ring, or snap gage, in the absence of information as to the number of threads per inch of the screw to be made, or for gage dimensions other than thread diameters, the tolerances for plain gages given in Table 31 may be used. This table contains recommended tolerances for classes $\mathrm{X}, \mathrm{Y}$, and $\mathrm{Z}$ 
TABLE 27.-Recommended uses for classes $X, Y$, and $Z$ gages

\begin{tabular}{|c|c|c|c|}
\hline Class of fit & Setting gage & Inspection gage & Working gage \\
\hline 1 & 2 & 3 & 4 \\
\hline $\begin{array}{l}\text { Class } 1 \text {, loose fit } \\
\text { Class } 2 \text {, free fit. } \\
\text { Class } 3 \text {, medium fit...- } \\
\text { Class } 4 \text {, close fit...... }\end{array}$ & 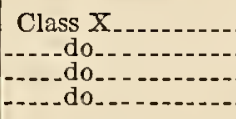 & $\begin{array}{l}\text { Class } Y \text {. } \\
\text { Class } \mathrm{X}\end{array}$ & $\begin{array}{l}\text { Class } Z . \\
\text { Class } Y . \\
\text { Do. } \\
\text { Class } \dot{X} .\end{array}$ \\
\hline
\end{tabular}

TABLE 28.-Tolerances for class $X$ "go" thread gages and all "not go" thread gages

\begin{tabular}{|c|c|c|c|c|c|c|}
\hline \multirow{2}{*}{ Threads per inch } & \multicolumn{2}{|c|}{$\begin{array}{l}\text { Tolerance on pitch } \\
\text { diameter }\end{array}$} & \multirow{2}{*}{$\begin{array}{l}\text { Tolerance } \\
\text { in lead }^{2}\end{array}$} & \multirow{2}{*}{$\begin{array}{c}\text { Tolerance } \\
\text { on half } \\
\text { angle of } \\
\text { thread }\end{array}$} & \multicolumn{2}{|c|}{$\begin{array}{l}\text { Tolerance on major or } \\
\text { minor diameters }\end{array}$} \\
\hline & From- & To- & & & From- & To- \\
\hline 1 & 2 & 3 & 4 & 5 & 6 & $\boldsymbol{y}$ \\
\hline $\begin{array}{l}80 \\
72 \\
64 \\
56 \\
48\end{array}$ & $\begin{array}{l}\text { Inch } \\
0.0000 \\
.0000 \\
.0000 \\
.0000 \\
.0000\end{array}$ & $\begin{array}{l}\text { Inch } \\
0.0002 \\
.0002 \\
.0002 \\
.0002 \\
.0002\end{array}$ & $\begin{array}{l}\text { Inch } \\
\pm \\
0.0002 \\
.0002 \\
.0002 \\
.0002 \\
.0002\end{array}$ & $\begin{array}{cc}\text { Deg. } & \text { Min. } \\
\text { 土 } & \\
0 & 30 \\
0 & 30 \\
0 & 30 \\
0 & 30 \\
0 & 30\end{array}$ & $\begin{array}{l}\text { Inch } \\
0.0000 \\
.0000 \\
.0000 \\
.0000 \\
.0000\end{array}$ & $\begin{array}{r}\text { Inch } \\
0.0003 \\
.0003 \\
.0004 \\
.0004 \\
.0004\end{array}$ \\
\hline $\begin{array}{l}44 \ldots \ldots \\
40 \ldots \ldots \\
36 \ldots \ldots \\
32 \ldots \ldots \\
28 \ldots \ldots\end{array}$ & $\begin{array}{l}.0000 \\
.0000 \\
.0000 \\
.0000 \\
.0000\end{array}$ & $\begin{array}{l}.0002 \\
.0002 \\
.0002 \\
.0003 \\
.0003\end{array}$ & $\begin{array}{l}.0002 \\
.0002 \\
.0002 \\
.0003 \\
.0003\end{array}$ & $\begin{array}{ll}0 & 20 \\
0 & 20 \\
0 & 20 \\
0 & 15 \\
0 & 15\end{array}$ & $\begin{array}{l}.0000 \\
.0000 \\
.0000 \\
.0000 \\
.0000\end{array}$ & $\begin{array}{l}.0004 \\
.0004 \\
.0004 \\
.0004 \\
.0005\end{array}$ \\
\hline 18 16 18 & $\begin{array}{l}.0000 \\
.0000 \\
.0000 \\
.0000 \\
.0000\end{array}$ & $\begin{array}{l}.0003 \\
.0003 \\
.0003 \\
.0003 \\
.0003\end{array}$ & $\begin{array}{l}.0003 \\
.0003 \\
.0003 \\
.0003 \\
.0003\end{array}$ & $\begin{array}{ll}0 & 15 \\
0 & 15 \\
0 & 10 \\
0 & 10 \\
0 & 10\end{array}$ & $\begin{array}{l}.0000 \\
.0000 \\
.0000 \\
.0000 \\
.0000\end{array}$ & $\begin{array}{l}.0005 \\
.0005 \\
.0005 \\
.0006 \\
.0006\end{array}$ \\
\hline $\begin{array}{l}13 \\
12 \\
11 \\
10\end{array}$ & $\begin{array}{l}.0000 \\
.0000 \\
.0000 \\
.0000 \\
.0000\end{array}$ & $\begin{array}{l}.0003 \\
.0003 \\
.0003 \\
.0003 \\
.0003\end{array}$ & $\begin{array}{l}.0003 \\
.0003 \\
.0003 \\
.0003 \\
.0003\end{array}$ & $\begin{array}{ll}0 & 10 \\
0 & 10 \\
0 & 10 \\
0 & 10 \\
0 & 10\end{array}$ & $\begin{array}{l}.0000 \\
.0000 \\
.0000 \\
.0000 \\
.0000\end{array}$ & $\begin{array}{l}.0006 \\
.0006 \\
.0006 \\
.0006 \\
.0007\end{array}$ \\
\hline $\begin{array}{l}8 \\
7 \\
6 \\
4 \\
6\end{array}$ & $\begin{array}{l}.0000 \\
.0000 \\
.0000 \\
.0000 \\
.0000 \\
.0000\end{array}$ & $\begin{array}{l}.0004 \\
.0004 \\
.0004 \\
.0004 \\
.0004 \\
.0004\end{array}$ & $\begin{array}{l}.0004 \\
.0004 \\
.0004 \\
.0004 \\
.0004 \\
.0004\end{array}$ & $\begin{array}{l}0 \\
0 \\
0 \\
0 \\
0 \\
0\end{array}$ & $\begin{array}{l}.0000 \\
.0000 \\
.0000 \\
.0000 \\
.0000 \\
.0000\end{array}$ & $\begin{array}{l}.0007 \\
.0007 \\
.0008 \\
.0008 \\
.0008 \\
.0009\end{array}$ \\
\hline
\end{tabular}

1 On "go" plugs the tolerance is plus, and on "go" rings the tolerance is minus. On "not go" plugs the tolerance is minus, and on "not go" rings the tolerance is plus.

2 Allowable variation in lead between any two threads not farther apart than the standard length of engagement, which is equal to the basic major diameter.

gages, which have been tentatively adopted by the A. E. S. C. Sectional Committee on the Standardization of Plain Limit Gages for General Engineering Work.

3. Recommended Gage Practice.-There are given in Table 27 the recommended uses for the foregoing classes of gages. Tables 32, 33, 34 , and 35 give limiting dimensions of gages of the several classifications for the "national coarse" and "national fine thread series." 
It is suggested that, in case of question between the manufacturer and purchaser of threaded products in regard to their size, if the manufacturer produces limit gages which do not measure outside of the specified limits for the threaded components and which pass the parts in question, they be accepted as meeting the specifications for size. In case the dimensions of the gages are questioned, their sizes shall be determined by a disinterested third party, preferably the Bureau of Standards at Washington, D. C., which maintains a departnent for this service.

4. Marking of Gages.-Gages should be plainly marked, for identification, with the diameter, pitch, and thread series. See Section II, division 2, "Symbols."

TABLE 29.-Tolerances for class $Y$ "go" thread gages

\begin{tabular}{|c|c|c|c|c|c|c|}
\hline \multirow{2}{*}{ Threads per inch } & \multicolumn{2}{|c|}{$\begin{array}{l}\text { Tolerance on pitch } \\
\text { diameter }\end{array}$} & \multirow{2}{*}{$\begin{array}{l}\text { Tolerance } \\
\text { in lead } 2\end{array}$} & \multirow{2}{*}{$\begin{array}{c}\text { Tolerance } \\
\text { on half } \\
\text { angle of } \\
\text { thread }\end{array}$} & \multicolumn{2}{|c|}{$\begin{array}{l}\text { Tolerance on major or } \\
\text { minor diameters } 1\end{array}$} \\
\hline & From- & To- & & & From- & To- \\
\hline 1 & 2 & 3 & 4 & 5 & 6 & 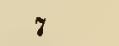 \\
\hline $\begin{array}{l}80 \ldots \ldots \\
72 . \ldots . . \\
64 \ldots \\
56 \ldots \ldots \\
48 \ldots \ldots\end{array}$ & $\begin{array}{l}\text { Inch } \\
0.0001 \\
.0001 \\
.0001 \\
.0001 \\
.0001\end{array}$ & $\begin{array}{l}\text { Inch } \\
0.0003 \\
.0003 \\
.0004 \\
.0004 \\
.0004\end{array}$ & $\begin{array}{l}\text { Inch } \\
\pm \\
0.0002 \\
.0002 \\
.0002 \\
.0002 \\
.0002\end{array}$ & $\begin{array}{ll}\text { Deg. } & \text { Min. } \\
\text { 土 } & \\
0 & 45 \\
0 & 45 \\
0 & 45 \\
0 & 45 \\
0 & 45\end{array}$ & $\begin{array}{l}\text { Inch } \\
0.0000 \\
.0000 \\
.0000 \\
.0000 \\
.0000\end{array}$ & $\begin{array}{r}\text { Inch } \\
0.0003 \\
.0003 \\
.0004 \\
.0004 \\
.0004\end{array}$ \\
\hline $\begin{array}{l}44 \ldots \ldots \\
40 \ldots \ldots \\
36 \ldots . . . \\
32 \\
28 \ldots . . . \\
\end{array}$ & $\begin{array}{l}.0001 \\
.0001 \\
.0001 \\
.0001 \\
.0002\end{array}$ & $\begin{array}{l}.0004 \\
.0004 \\
.0004 \\
.0004 \\
.0005\end{array}$ & $\begin{array}{l}.0002 \\
.0002 \\
.0002 \\
.0003 \\
.0003\end{array}$ & $\begin{array}{ll}0 & 30 \\
0 & 30 \\
0 & 30 \\
0 & 20 \\
0 & 20\end{array}$ & $\begin{array}{l}.0000 \\
.0000 \\
.0000 \\
.0000 \\
.0000\end{array}$ & $\begin{array}{l}.0004 \\
.0004 \\
.0004 \\
.0004 \\
.0005\end{array}$ \\
\hline $\begin{array}{l}24 \ldots \ldots \\
20 . \ldots . \\
18 \\
16 \\
14 \ldots . . . \\
14 . \ldots\end{array}$ & $\begin{array}{l}.0002 \\
.0002 \\
.0002 \\
.0002 \\
.0002\end{array}$ & $\begin{array}{l}.0005 \\
.0005 \\
.0005 \\
.0006 \\
.0006\end{array}$ & $\begin{array}{l}.0003 \\
.0003 \\
.0003 \\
.0003 \\
.0003\end{array}$ & $\begin{array}{ll}0 & 20 \\
0 & 20 \\
0 & 15 \\
0 & 15 \\
0 & 15\end{array}$ & $\begin{array}{l}.0000 \\
.0000 \\
.0000 \\
.0000 \\
.0000\end{array}$ & $\begin{array}{l}.0005 \\
.0005 \\
.0005 \\
.0006 \\
.0006\end{array}$ \\
\hline $\begin{array}{r}13 . \ldots \\
12 \ldots \\
11 . . \\
10 . . \\
9 . .\end{array}$ & $\begin{array}{l}.0002 \\
.0002 \\
.0002 \\
.0002 \\
.0002\end{array}$ & $\begin{array}{l}.0006 \\
.0006 \\
.0006 \\
.0006 \\
.0007\end{array}$ & $\begin{array}{l}.0003 \\
.0003 \\
.0003 \\
.0003 \\
.0003\end{array}$ & $\begin{array}{l}15 \\
10 \\
10 \\
10 \\
10\end{array}$ & $\begin{array}{l}.0000 \\
.0000 \\
.0000 \\
.0000 \\
.0000\end{array}$ & $\begin{array}{l}.0006 \\
.0006 \\
.0006 \\
.0006 \\
.0007\end{array}$ \\
\hline $\begin{array}{l}8 \\
7 \\
6 \\
4 \\
4\end{array}$ & $\begin{array}{l}.0002 \\
.0002 \\
.0003 \\
.0003 \\
.0003 \\
.0003\end{array}$ & $\begin{array}{l}.0007 \\
.0007 \\
.0008 \\
.0008 \\
.0008 \\
.0009\end{array}$ & $\begin{array}{l}.0004 \\
.0004 \\
.0004 \\
.0004 \\
.0004 \\
.0004\end{array}$ & $\begin{array}{l}0 \\
0 \\
0 \\
0 \\
0 \\
0\end{array}$ & $\begin{array}{l}.0000 \\
.0000 \\
.0000 \\
.0000 \\
.0000 \\
.0000\end{array}$ & $\begin{array}{l}.0007 \\
.0007 \\
.0008 \\
.0008 \\
.0008 \\
.0009\end{array}$ \\
\hline
\end{tabular}

1 On "go" plugs the tolerance is plus and on "go" rings the tolerance is minus.

Allowable variation in lead between any two threads not farther apart than the standard length of engagement, which is equal to the basic major diameter. 
1924 REPORT

TABLE 30.-Tolerances for class $Z$ "go" thread gages

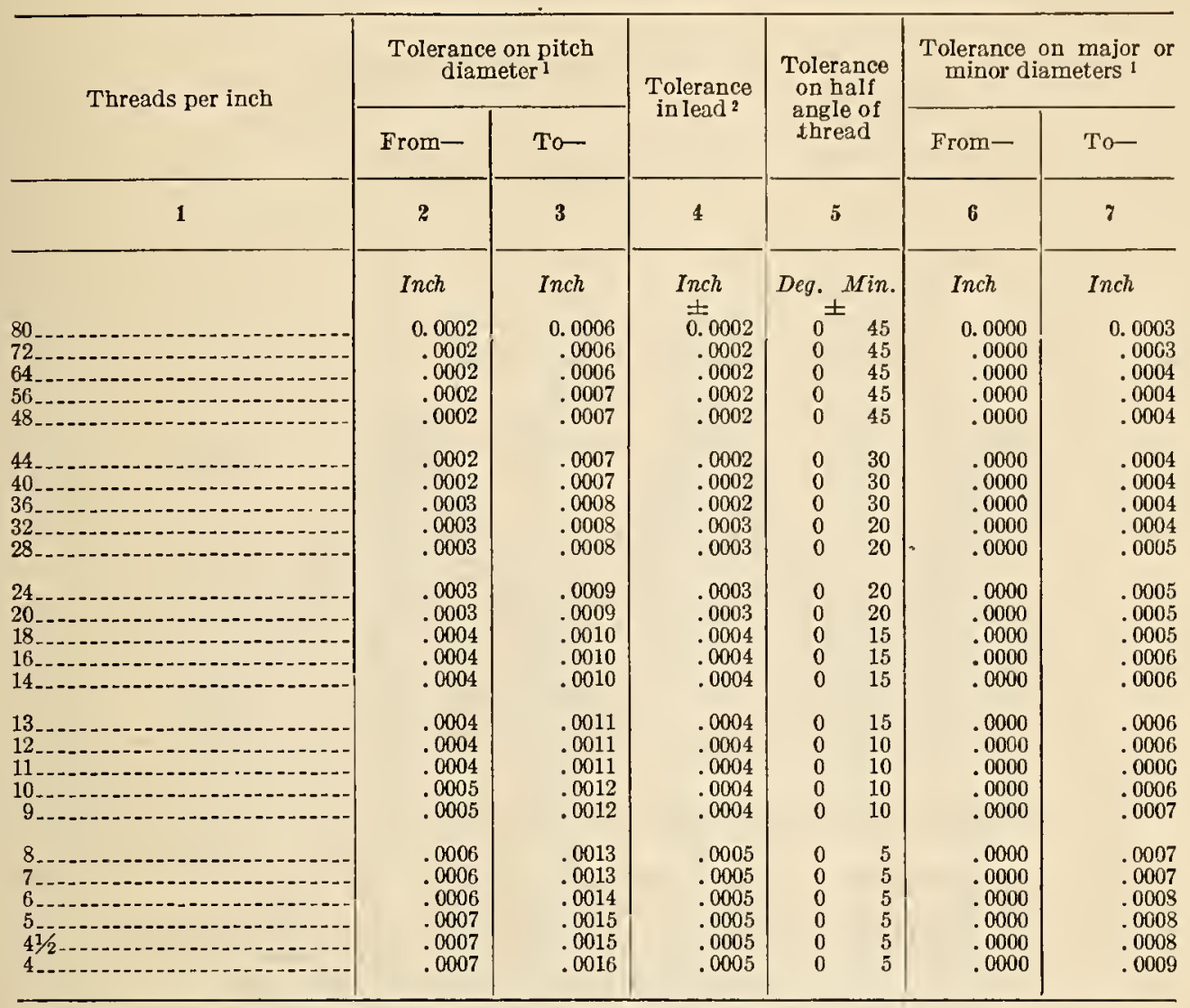

1 On "go" plugs the tolerance is plus, and on "go" rings the tolerance is minus.

2 Allowable variation in lead between any two threads not farther apart than the standard length of engagement, which is equal to the basic major diameter.

TABLE 31.-Tolerances for plain gages 1

\begin{tabular}{|c|c|c|c|c|c|c|}
\hline \multirow{2}{*}{ Size of gage in inches } & \multicolumn{2}{|c|}{ Class $\mathrm{X}^{2}$} & \multicolumn{2}{|c|}{ Class Y } & \multicolumn{2}{|c|}{ Class Z } \\
\hline & From- & To- & From- & To- & From- & To- \\
\hline 1 & 2 & 3 & 4 & 5 & 6 & 7 \\
\hline $\begin{array}{l}0 \text { to } 1 \text {, inclusive } \\
1 \text { to } 3 \text {, inclusive }\end{array}$ & $\begin{array}{l}\text { Inch } \\
0.0000 \\
.0000\end{array}$ & $\begin{array}{l}\text { Inch } \\
0.0001 \\
.0002\end{array}$ & $\begin{array}{l}\text { Inch } \\
0.0001 \\
.0001\end{array}$ & $\begin{array}{l}\text { Inch } \\
0.0002 \\
.0003\end{array}$ & $\begin{array}{l}\text { Inch } \\
0.0002 \\
.0003\end{array}$ & $\begin{array}{l}\text { Inch } \\
0.0003 \\
.0005\end{array}$ \\
\hline
\end{tabular}

1 On "go" plugs the tolerance is plus, and on "go" rings the tolerance is minus. On "not go" plugs the tolerance is minus, and on "not go" rings the tolerance is plus.

2 All "not go" gages are made to class $\mathrm{X}$ tolerances.

$16802^{\circ}-25 \div-6$ 


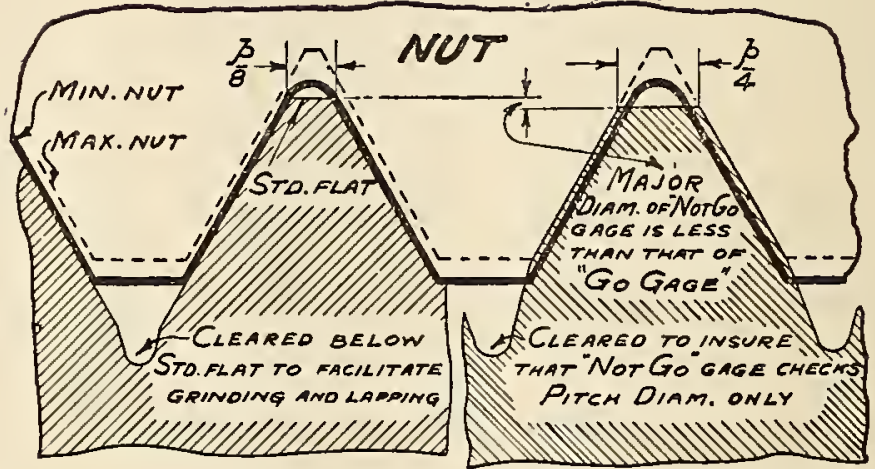

Minimum or "Go" Plug Gage Maximum op"NotGo"PlugGage Maximum or "Go'RING GaGe Minimum op"Not Go"RING Gage

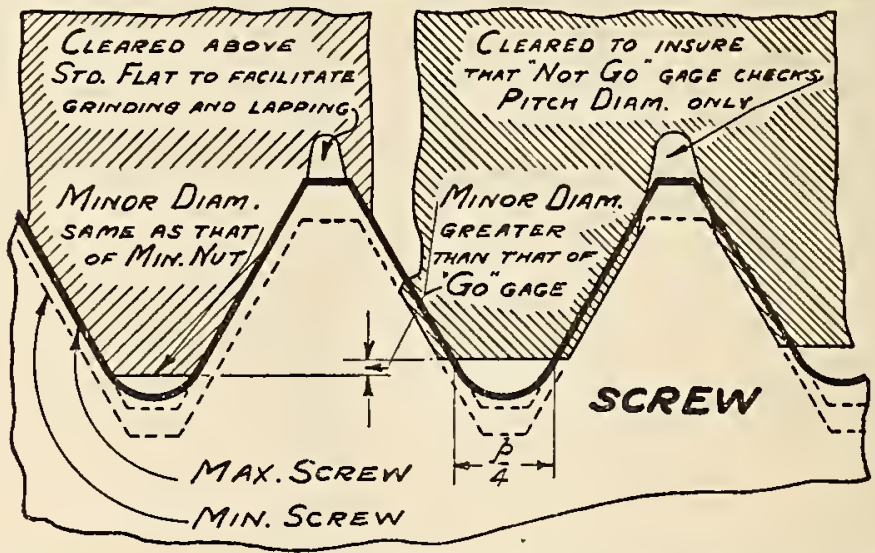

FIG. 22.-Thread form of "go" and "not go" thread plug and ring gages Note.- "Not go" gages check pitch diameter only. 


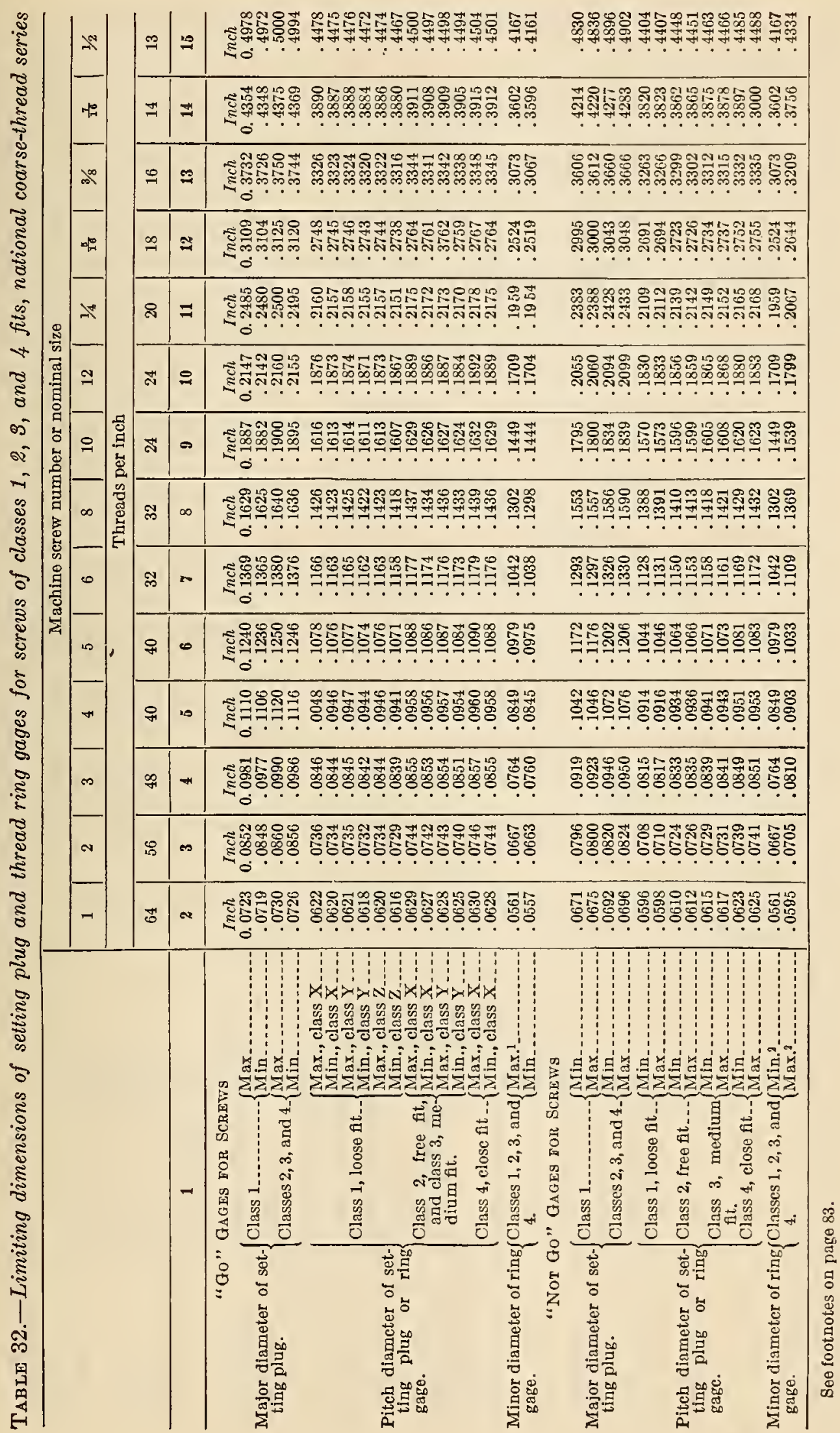




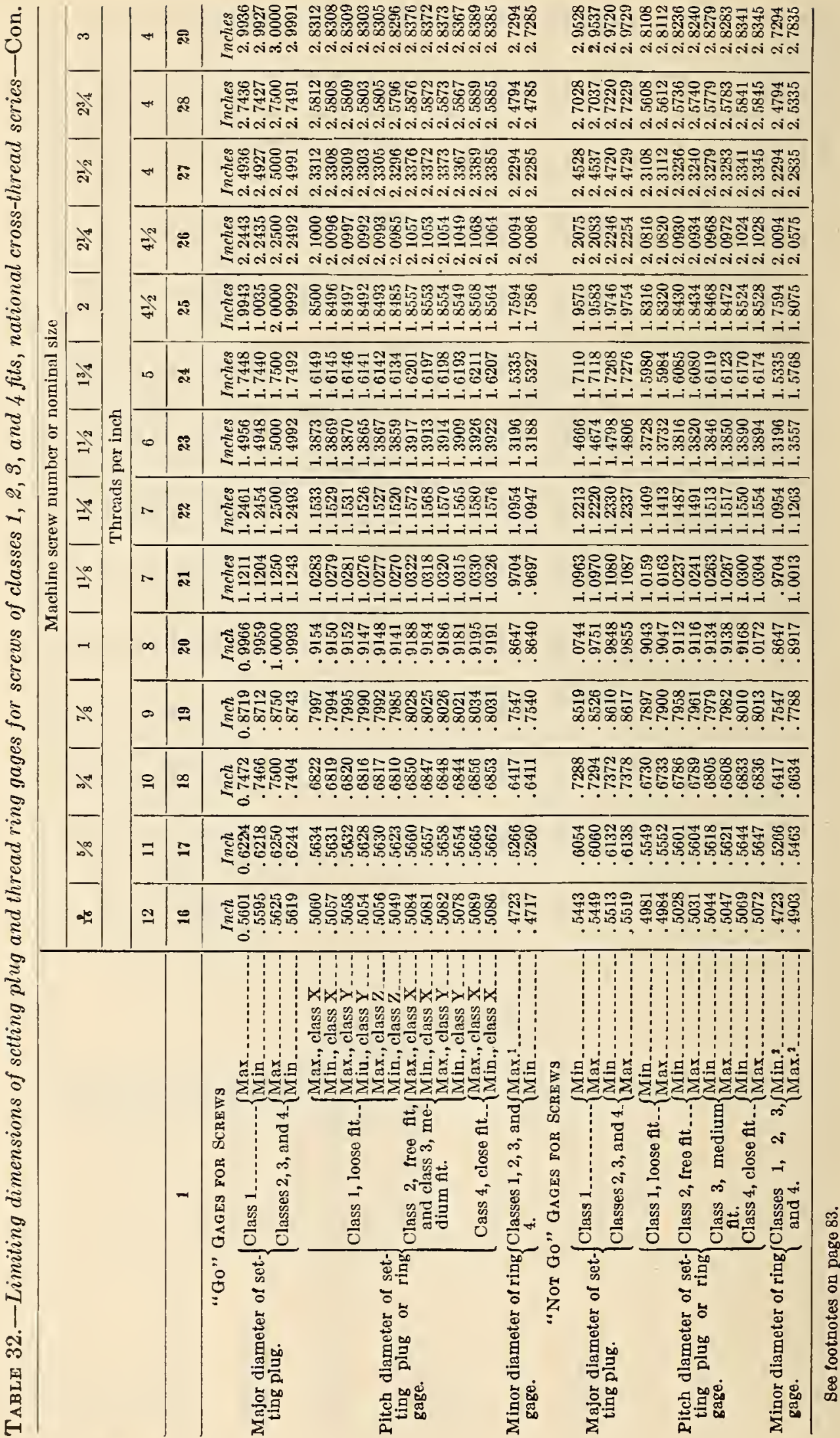




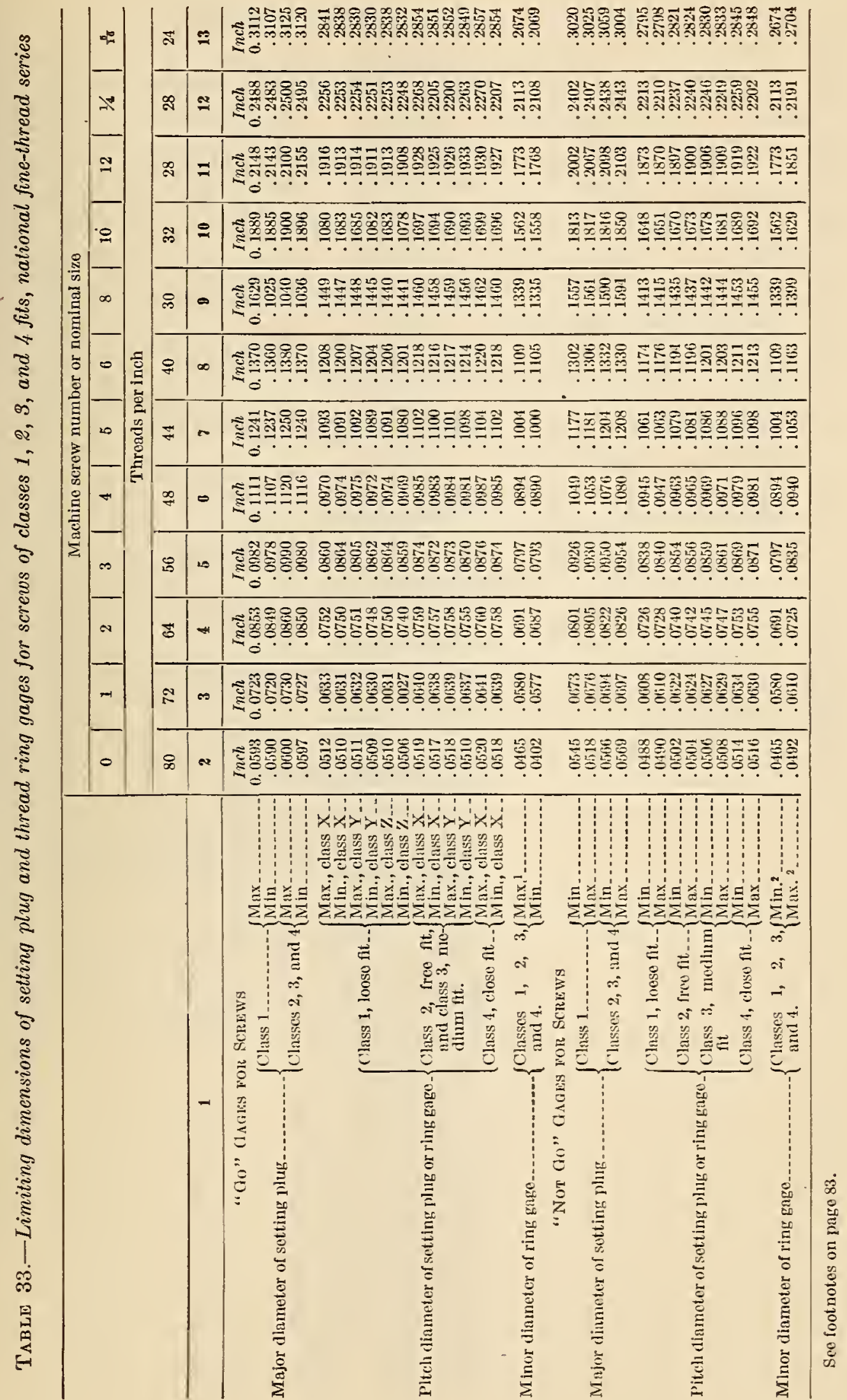




\begin{tabular}{|c|c|c|c|c|c|c|c|}
\hline \multirow{12}{*}{ 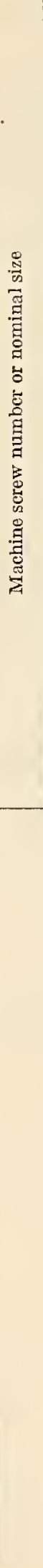 } & $\Rightarrow$ & \multirow{11}{*}{ 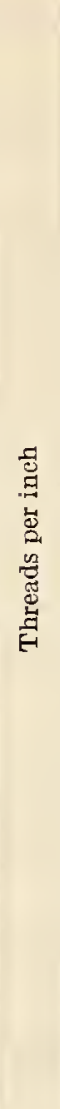 } & F & $\vec{x}$ & 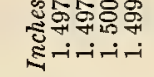 & 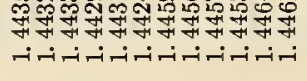 & 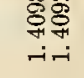 \\
\hline & 라 & & $\approx$ & : & 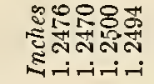 & 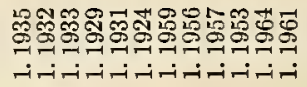 & 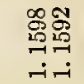 \\
\hline & $\Rightarrow$ & & $\cong$ & $\stackrel{20}{*}$ & 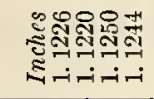 & 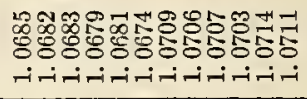 & 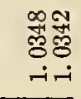 \\
\hline & $r$ & & $\exists$ & $\vec{\nabla}$ & 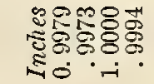 & 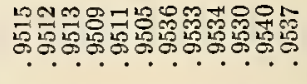 & ్ํำ \\
\hline & $\infty$ & & 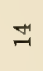 & $\stackrel{P}{*}$ & 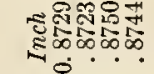 & 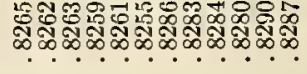 & \\
\hline & - & & I & $\nexists$ & 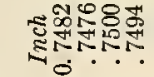 & 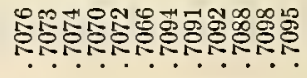 & \\
\hline & $\infty$ & & $\stackrel{\infty}{\sim}$ & 2 & ₹ & 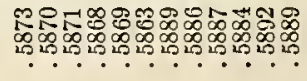 & \\
\hline & 怗 & & $\stackrel{\infty}{\sim}$ & $\approx$ & 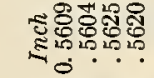 & 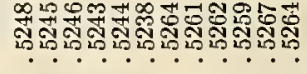 & 藏官 \\
\hline & $\lambda^{\alpha}$ & & సิ & 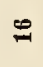 & 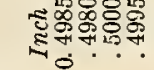 & 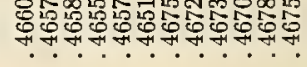 & \\
\hline & $H^{\circ}$ & & ని & 20 & 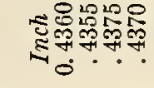 & 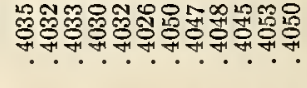 & 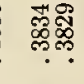 \\
\hline & $\infty$ & & $\vec{a}$ & $\exists$ & 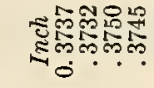 & 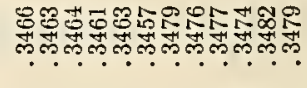 & 跗芯 \\
\hline & & & & -1 & 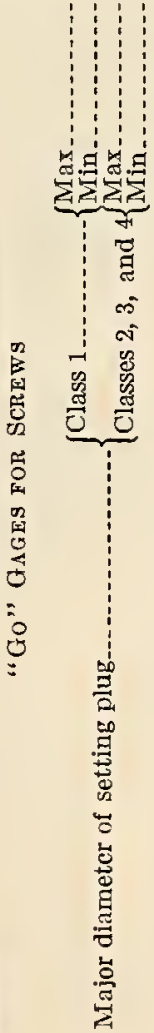 & 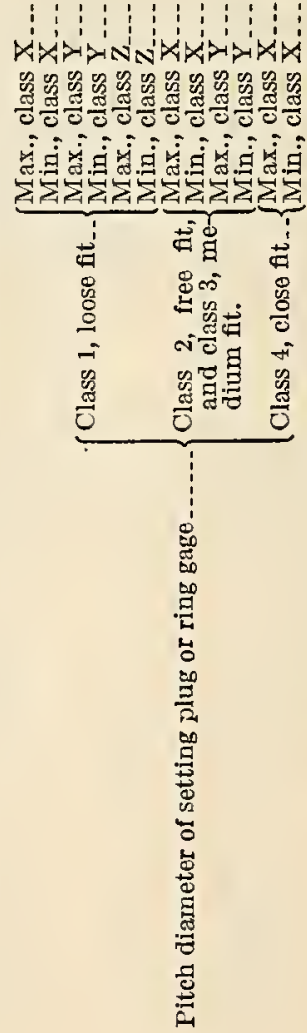 & 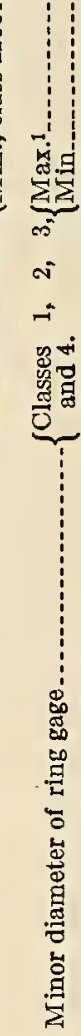 \\
\hline
\end{tabular}




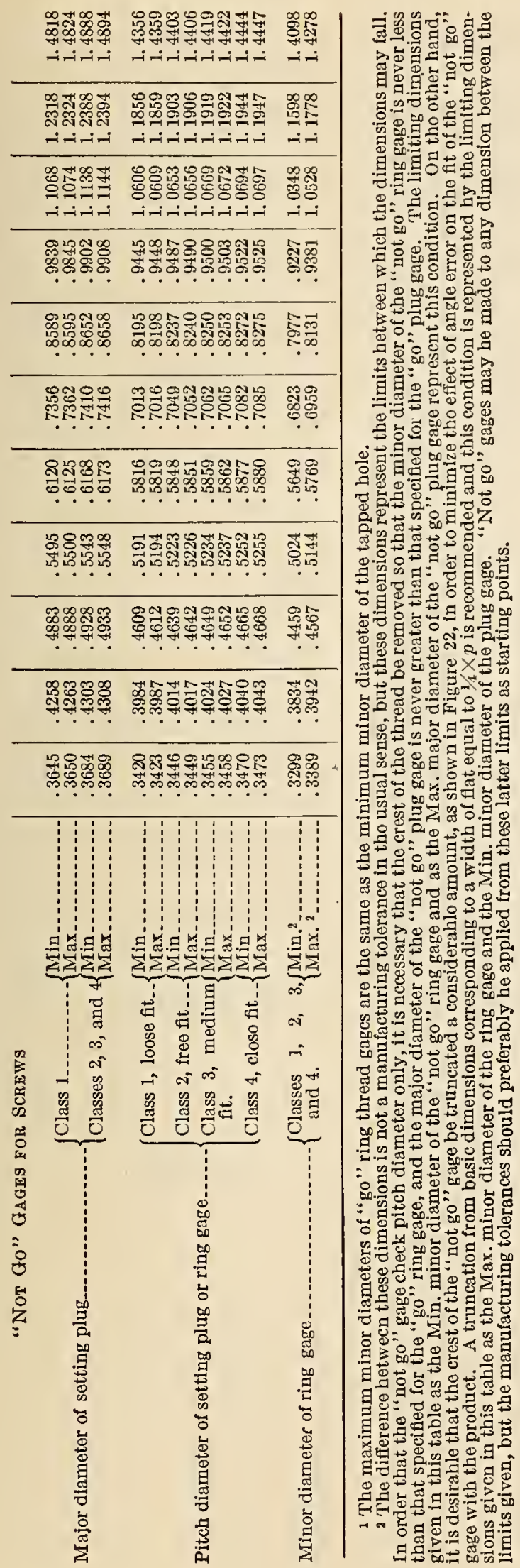


1924 REPORT

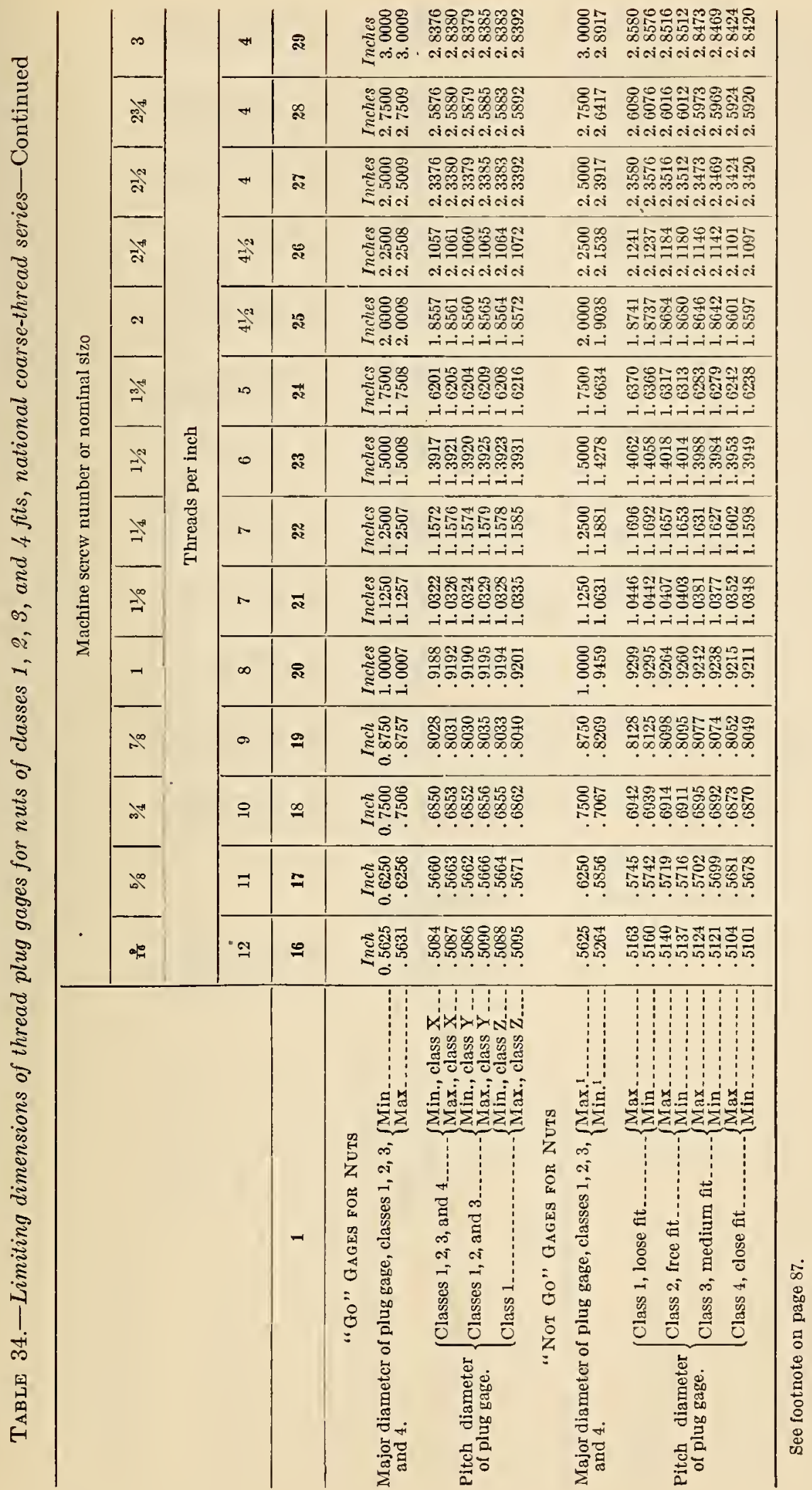




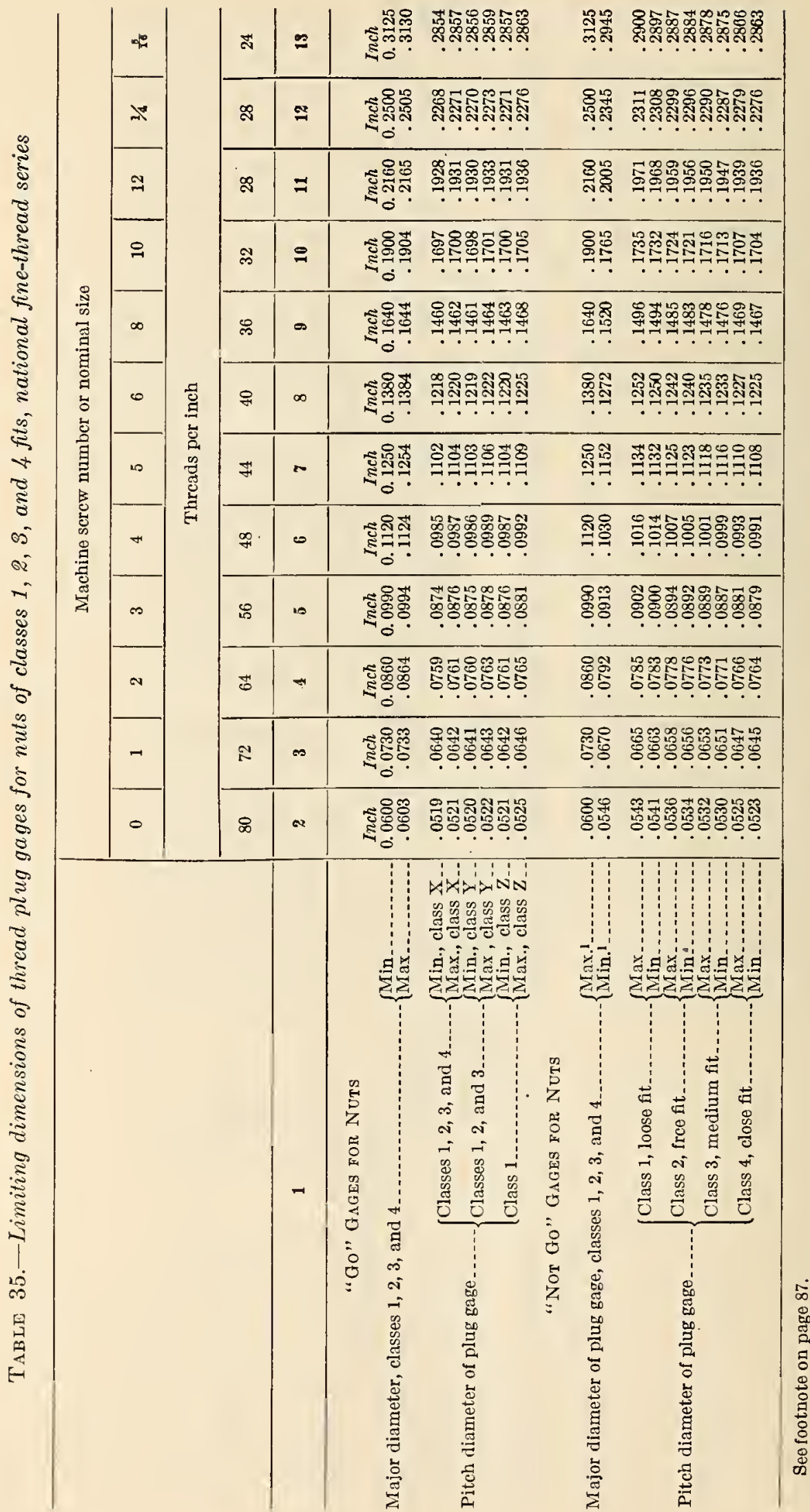


1924 REPORT

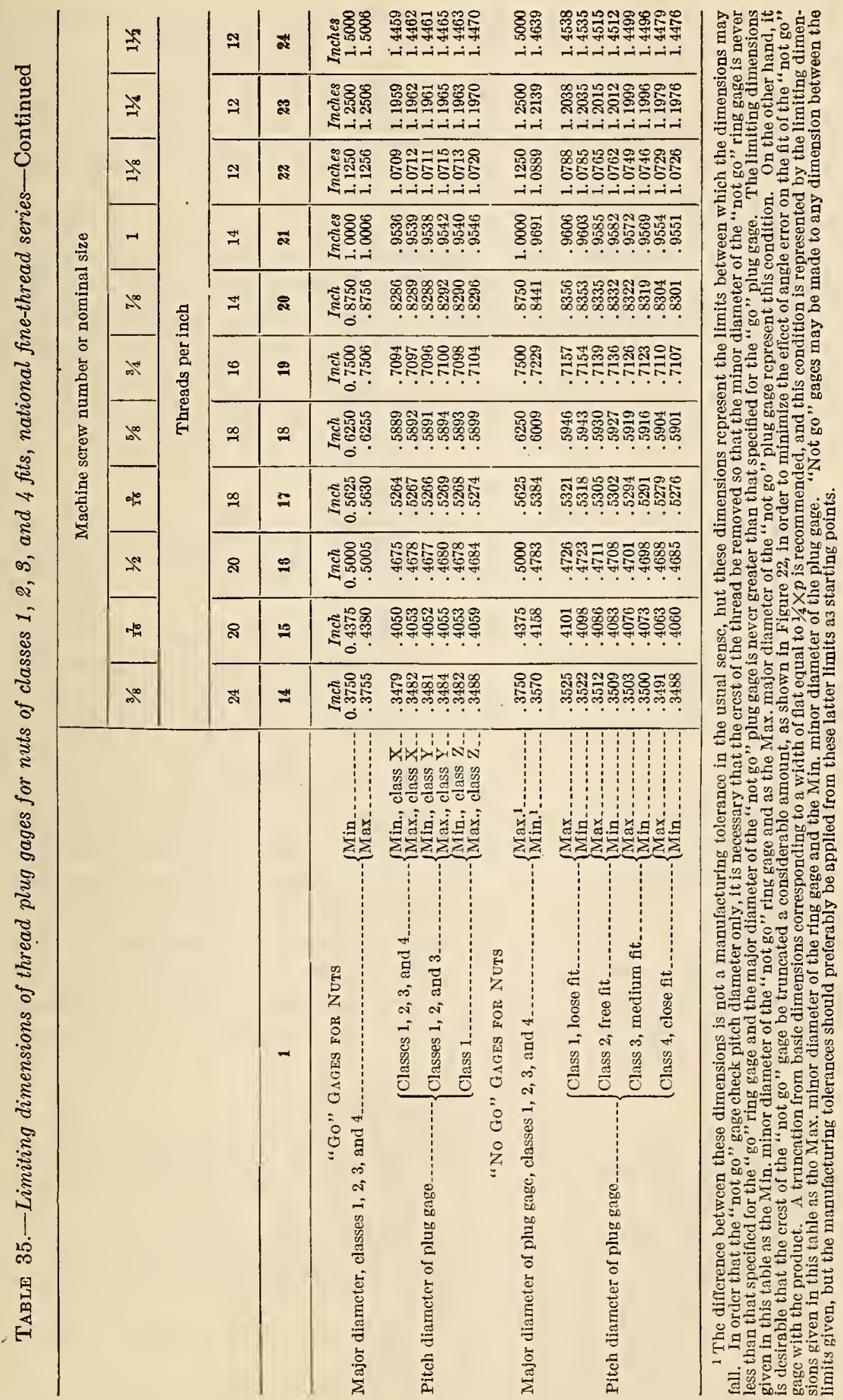




\section{SECTION IV. SCREW THREADS OF SPECIAL DIAMETERS, PITCHES, AND LENGTHS OF ENGAGEMENT}

The tolerances specified in Section III of this report are based on the pitch of the threads only, and apply, in general, to bolts, nuts, etc., of standard pitches and diameters and for lengths of engagement not greater than the diameter.

In addition to the foregoing threaded components, there are large quantities of threaded parts produced, such as hub and radiator caps in the automotive industry, threaded collars on machine tools, etc., where the diameters are larger, the pitches finer, and the lengths of engagements shorter than for bolt and nut practice. The following specifications have been adopted for such threaded parts, taking into consideration, when determining the tolerances, the diameter, pitch, and length of engagement of the particular components.

\section{FORM OF THREAD}

The national form of thread profile as specified in Section III, and known previously as the United States form or Sellers' profile, shall be used.

\section{THREAD SERIES}

It is recommended that one of the following pitches be selected whenever it is necessary to use a screw thread of special diameter and pitch: $4,6,8,10,12,14,16,18,20,24,28,32,36,40,48,56,64$.

\section{CLASSIFICATION AND TOLERANCES}

There are established herein for general use five distinct classes of screw-thread fits, which are lettered to distinguish them from the regular classification of fits. These five classes, together with the accompanying specifications, are for the purpose of insuring a uniform practice on screw-thread production throughout the country.

It is not the intention of the commission to arbitrarily place a general class or grade of work in a specific class of fit. Each manufacturer and user of screw threads is free to select the class of fit best adapted to his particular needs.

\section{(a) GENERAL SPECIFICATIONS}

The following general specifications apply to all classes of fit specified for screw threads of special diameters, pitches, and lengths of engagement.

1. Uniform Mrnimum Nut.-The pitch diameter of the minimum threaded hole or nut corresponds to the basic size, the tolerances being applied above the basic size.

2. Tolerancess. ${ }^{9}-(a)$ The tolerances specified represent the extreme variations allowed on the product.

- Recommendations and explanations regarding the application of tolerances are given in Appendix 1. 
(b) The tolerance on the nut is plus, and is applied from the basic size to above basic size.

(c) The tolerance on the screw is minus, and is applied from the maximum screw size to below the maximum screw size.

(d) The pitch diameter tolerances for a screw and nut of a given class of fit are the same.

(e) Pitch diameter tolerances include lead and angle variations.

(f) The pitch diameter tolerances are obtained by adding three values, or increments, one dependent upon the basic major diameter, another upon the iength of engagement, and the third upon the pitch of the thread. These increments are based on formulas given under "Classification of fits."

(g) The tolerances on the major diameters of the screws and minor diameters of the nuts are based on the pitch of the thread, as these control the depth of engagement; they are, therefore, based on the pitch alone.

(h) The minimum minor diameter of a screw of a given pitch is such as to result in a basic flat $(1 / 8 \times p)$ at the root when the pitch diameter of the screw is at its minimum value. When the maximum screw is basic, the minimum minor diameter of the screw will be below the basic minor diameter by the amount of the specified pitch diameter tolerance.

(i) The maximum minor diameter of a screw of a given pitch may be such as results from the use of a worn or rounded threading tool, when the pitch diameter is at its maximum value. In no case, however, should the form of the screw, as results from tool wear, be such as to cause the screw to be rejected on the maximum minor diameter by a "go" ring gage, the minor diameter of which is equal to the minimum minor diameter of the nut.

(j) The maximum major diameter of the nut of a given pitch is such as to result in a flat one-third of the basic flat $\left(\frac{1}{24} \times p\right)$ when the pitch diameter of the nut is at its maximum value. When the minimum nut is basic, its maximum major diameter will be above the basic major diameter by the amount of the specified pitch diameter tolerance plus two-ninths of the basic thread depth.

(k) The nominal minimum major diameter of a nut is the basic major diameter. In no case, however, should the minimum major diameter of the nut, as results from a worn tap or cutting tool, be such as to cause the nut to be rejected on the minimum major diameter by a "go" plug gage made to the standard form at the crest.

(l) The tolerance on minor diameter of a nut of a given pitch is one-sixth of the basic thread depth regardless of the class of fit being produced. 


\section{(b) CLASSIFICATION OF FITS}

1. Class A.-This class is intended to cover the manufacture of threaded parts where quick and easy assembly is necessary and a considerable amount of shake or play is not objectionable.

This class is made with an allowance on the screw, so as to permit ready assembly, even when the threads are slightly bruised or dirty, in conformity with the practice in Section III.

Special cases will arise, however, when a class A thread is required on finished drawn tubing with thin walls, and in such cases the allowance should be made on the nut.

The pitch diameter tolerances are obtained by adding three values, or increments, as follows:

where

$$
\begin{array}{lrr}
\text { Diameter increment } & =0.002 \sqrt{D} \\
\text { Length of engagement increment } & =.002 \\
\text { Pitch increment } & =.020 & \sqrt{p}
\end{array}
$$

$$
\begin{aligned}
& \dot{D}=\text { basic major diameter } \\
& Q=\text { length of engagement } \\
& p=\text { pitch of thread in iuches. }
\end{aligned}
$$

Tables 36,37 , and 38 give the clearances and tolerances for major and minor diameters, and tolerance increments for pitch diameters for a range of diameters, lengths of engagement, and pitches. The total pitch diameter tolerance may be obtained by adding increments obtained from the tables, or it may be obtained directly from the chart, Figure 23.

Example: $31 / 4$-inch, 16-thread, class A, with allowance on screw, one-half inch length of engagement.

From Table 36 we get for the screw:

Maximum major diameter $=3.250-0.0018=3.2482$

Minimum major diameter $=3.2482-.0126=3.2356$

Maximum minor diameter $=3.2482-.0767=3.1715$

Maximum pitch diameter $=3.2482-.0406=3.2076$

And for the nut:

Minimum major diameter $\quad=3.250$

Minimum minor diameter $=3.250-.0677=3.1823$

Maximum minor diameter $=3.1823+.0068=3.1891$

Minimum pitch diameter $=3.250-.0406=3.2094$

Also for the pitch diameter tolerance:

$$
\text { Pitch increment }=.00500
$$

From Table 37, diameter increment $(31 / 4$ inches $)=.00360$ (by interpolation)

From Table 38, engagement increment $(1 / 2$ inch $)=.00100$

$$
\text { Total }
$$$$
.00960
$$

Increment values are given to five decimal places for purposes of calculation. The final result may be rounded off to the nearest third 
or fourth decimal place. The pitch diameter tolerance in this example for both screw and nut can be 0.0096 , or 0.010 inch.

The pitch diameter tolerance should never be appreciably greater than the tolerance on the major diameter of the screw. If the cal-

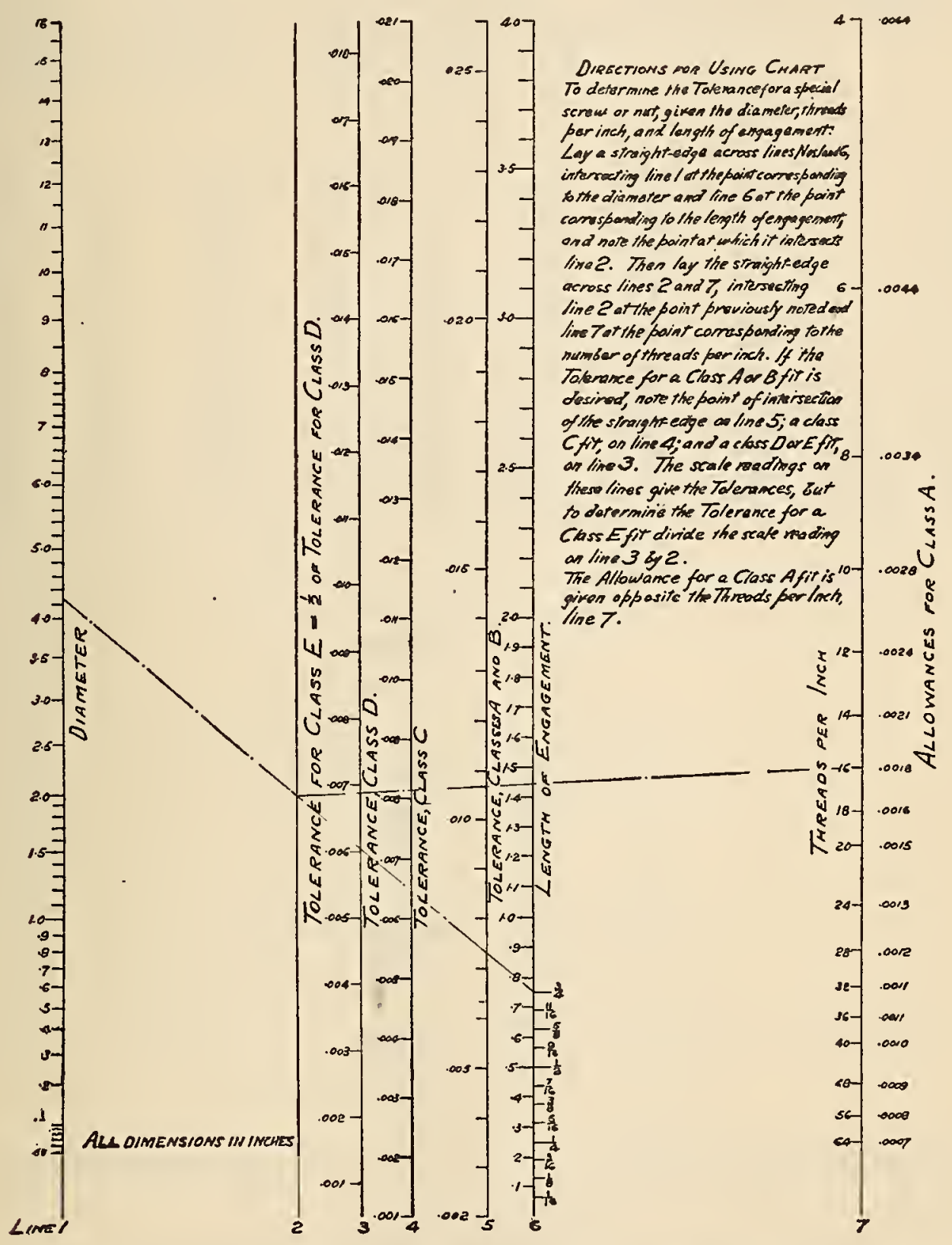

FIG. 23.-Chart for determining tolerances for various classes of fit of special threads

culated pitch diameter tolerance exceeds the tolerance on the major diameter of the screw, it is evident that too fine a pitch or too loose a fit has been selected for the particular service. The foregoing example should be sufficient to illustrate the use of thcse tables. 
TABLE 36.-Class A, allowances and pitch increments

\begin{tabular}{|c|c|c|c|c|c|c|c|c|}
\hline \multirow[b]{2}{*}{$\begin{array}{l}\text { Number of } \\
\text { threads per } \\
\text { inch }\end{array}$} & \multirow[b]{2}{*}{$\begin{array}{l}\text { Allow- } \\
\text { ance }\end{array}$} & \multirow[b]{2}{*}{$\begin{array}{c}\text { Pitch } \\
\text { diameter } \\
\text { tolerance, } \\
\text { pitch } \\
\text { increment }\end{array}$} & \multicolumn{3}{|c|}{ Screw } & \multicolumn{3}{|c|}{ Nut } \\
\hline & & & $\begin{array}{c}\text { Tolerance } \\
\text { on } \\
\text { major } \\
\text { diameter, } \\
\text { minus }\end{array}$ & $\begin{array}{l}\text { Maximum } \\
\text { minor } \\
\text { diameter }= \\
\text { maximum } \\
\text { major } \\
\text { diameter } \\
\text { minus }\end{array}$ & $\begin{array}{c}\text { Maximum } \\
\text { pitch } \\
\text { diameter }= \\
\text { maximum } \\
\text { major } \\
\text { diameter } \\
\text { minus }\end{array}$ & $\begin{array}{c}\text { Tolerance } \\
\text { on } \\
\text { minor } \\
\text { diameter, } \\
\text { plus }\end{array}$ & $\begin{array}{l}\text { Minimum } \\
\text { minor } \\
\text { diameter }= \\
\text { basic } \\
\text { major } \\
\text { diameter } \\
\text { minus }\end{array}$ & $\begin{array}{l}\text { Minimum } \\
\text { pitch } \\
\text { diameter = } \\
\text { minimum } \\
\text { major } \\
\text { diameter } \\
\text { minus }\end{array}$ \\
\hline 1 & 2 & 3 & 4 & 5 & 6 & 7 & 8 & 9 \\
\hline $\begin{array}{l}64 \ldots \\
56 \\
40 \\
36\end{array}$ & $\begin{array}{l}\text { Inch } \\
0.0007 \\
.0008 \\
.0009 \\
.0010 \\
.0011\end{array}$ & $\begin{array}{l}\text { Inch } \\
0.00250 \\
.00267 \\
.00289 \\
.00316 \\
.00333\end{array}$ & $\begin{array}{l}\text { Inch } \\
0.0052 \\
.0056 \\
.0062 \\
.0068 \\
.0072\end{array}$ & $\begin{array}{l}\text { Inch } \\
0.0192 \\
.0219 \\
.0256 \\
.0307 \\
.0341\end{array}$ & $\begin{array}{l}\text { Inch } \\
0.0101 \\
.0116 \\
.0135 \\
.0162 \\
.0180\end{array}$ & $\begin{array}{l}\text { Inch } \\
0.0017 \\
.0019 \\
.0023 \\
.0027 \\
.0030\end{array}$ & $\begin{array}{l}\text { Inch } \\
0.0169 \\
.0193 \\
.0226 \\
.0271 \\
.0301\end{array}$ & $\begin{array}{l}\text { Inch } \\
0.0101 \\
.0116 \\
.0135 \\
.0162 \\
.0180\end{array}$ \\
\hline $\begin{array}{l}32 \\
28 \ldots \ldots \ldots \\
24 \ldots \ldots \\
20 \ldots \ldots \ldots \\
18 \ldots \ldots \ldots \ldots \ldots\end{array}$ & $\begin{array}{l}.0011 \\
.0012 \\
.0013 \\
.0015 \\
.0016\end{array}$ & $\begin{array}{l}.00354 \\
.00378 \\
.00408 \\
.00447 \\
.00471\end{array}$ & $\begin{array}{l}.0076 \\
.0086 \\
.0092 \\
.0102 \\
.0114\end{array}$ & $\begin{array}{l}.0383 \\
.0438 \\
.0511 \\
.0613 \\
.0682\end{array}$ & $\begin{array}{l}.0203 \\
.0232 \\
.0271 \\
.0325 \\
.0361\end{array}$ & $\begin{array}{l}.0034 \\
.0039 \\
.0045 \\
.0054 \\
.0060\end{array}$ & $\begin{array}{l}.0338 \\
.0387 \\
.0451 \\
.0541 \\
.0601\end{array}$ & $\begin{array}{l}.0203 \\
.0232 \\
.0271 \\
.0325 \\
.0361\end{array}$ \\
\hline $\begin{array}{l}16 \\
14 \\
12 \\
10 \\
8\end{array}$ & $\begin{array}{l}.0018 \\
.0021 \\
.0024 \\
.0028 \\
.0034\end{array}$ & $\begin{array}{l}.00500 \\
.00535 \\
.00577 \\
.00632 \\
.00707\end{array}$ & $\begin{array}{l}.0126 \\
.0140 \\
.0158 \\
.0184 \\
.0222\end{array}$ & $\begin{array}{l}.0767 \\
.0876 \\
.1022 \\
.1227 \\
.1534\end{array}$ & $\begin{array}{l}.0406 \\
.0464 \\
.0541 \\
.0650 \\
.0812\end{array}$ & $\begin{array}{l}.0068 \\
.0077 \\
.0090 \\
.0108 \\
.0135\end{array}$ & $\begin{array}{l}.0677 \\
.0773 \\
.0902 \\
.1083 \\
.1353\end{array}$ & $\begin{array}{l}.0406 \\
.0464 \\
.0541 \\
.0650 \\
.0812\end{array}$ \\
\hline $\begin{array}{l}6 \\
4 \\
4\end{array}$ & $\begin{array}{l}.0044 \\
.0064\end{array}$ & $\begin{array}{l}.00816 \\
.01000\end{array}$ & $\begin{array}{l}.0290 \\
.0408\end{array}$ & $\begin{array}{r}.2045 \\
.3067\end{array}$ & $\begin{array}{l}.1083 \\
.1624\end{array}$ & $\begin{array}{l}.0180 \\
.0271\end{array}$ & $\begin{array}{l}.1804 \\
.2706\end{array}$ & $\begin{array}{l}.1083 \\
.1624\end{array}$ \\
\hline
\end{tabular}

TABLE 37.-Class A, diameter increments of pitch diameter tolerances

\begin{tabular}{|c|c|}
\hline Diameter & $\begin{array}{c}\text { Diameter } \\
\text { increment }\end{array}$ \\
\hline 1 & 2 \\
\hline Inches & Inch \\
0.0625 & 0.00050 \\
.125 & .00071 \\
.1875 & .00087 \\
.250 & .00100 \\
.375 & .00122 \\
.500 & .00141 \\
.750 & .00173 \\
1.000 & .00200 \\
1.500 & .00245 \\
2.000 & .00283 \\
3.000 & .00346 \\
4.000 & .00400 \\
6.000 & .00490 \\
8.000 & .00565 \\
12.000 & .00693 \\
\hline
\end{tabular}

TABLE 38.-Class A, length of engagement increments of pitch diameter tolerances

\begin{tabular}{|c|c|}
\hline $\begin{array}{c}\text { Lenth of } \\
\text { engagement }\end{array}$ & Increment \\
\hline 1 & 2 \\
\hline Inches & Inch \\
0.250 & 0.00050 \\
.500 & .00100 \\
1.000 & .00000 \\
2.000 & .00400 \\
3.000 & .00600 \\
4.000 & .00800 \\
\hline
\end{tabular}


2. Class B.-This class is intended to cover the manufacture of threaded parts which are to assemble nearly, or entirely, with the fingers, and a moderate amount of shake or play is not objectionable; and no allowance is required. It is the same in every particular as class $\mathrm{A}$, except that it has no allowance.

The pitch diameter tolerances are the same as for class $\mathrm{A}$ and are obtained by adding three values, or increments, as follows:

where

$$
\begin{array}{ll}
\text { Diameter increment } & =0.002 \sqrt{D} \\
\text { Length of engagement increment } & =.002 Q \\
\text { Pitch increment } & =.020 \sqrt{p}
\end{array}
$$

$$
\begin{aligned}
& D=\text { basic major diameter } \\
& Q=\text { length of engagement } \\
& p=\text { pitch of thread in inches. }
\end{aligned}
$$

Tables 39, 40, and 41 give the tolerances for major and minor diameters, and tolerance increments for pitch diameters for a range of diameters, lengths of engagement, and pitches. The total pitch diameter tolerance may be obtained by adding increments obtained from the tables, or it may be obtained directly from the chart, Figure 23.

Example: $31 \frac{1}{4}$-inch, 16 thread, class B, one-half inch length of engagement.

From Table 39 we get for the screw:

Maximum major diameter $\quad=3.250$

Minimum major diameter $=3.250-0.0126=3.2374$

Maximum minor diameter $=3.250-.0767=3.1733$

Maximum pitch diameter $=3.250-.0406=3.2094$

And for the nut:

Minimum major diameter $\quad=3.250$

Minimum minor diameter $=3.250-.0677=3.1823$

Maximum minor diameter $=3.1823+.0068=3.1891$

Minimum pitch diameter $=3.250-.0406=3.2094$

Also for the pitch diameter tolerance:

Pitch increment $=.00500$

From Table 40, diameter increment (31/4inches) $=.00360$ (by interpolation)

From Table 41 , engagement increment $(1 / 2 \mathrm{inch})=.00100$ Total

.00960

Increment values are given to five decimal places for purposes of calculation. The final result may be rounded off to the nearest third or fourth decimal place. The pitch diameter tolerance in this example for both screw and nut can be 0.0096 , or 0.010 inch.

The pitch diameter tolerance should never be appreciably greater than the tolerance on the major diameter of the screw. If the calculated pitch diameter tolerance exceeds the tolerance on the major diameter of the screw, it is evident that too fine a pitch or too loose a fit has been selected for the particular service. The foregoing example should be sufficient to illustrate the use of these tables. $16802^{\circ}-25 \dagger-7$ 
TABLE 39.-Class B, pitch increments

\begin{tabular}{|c|c|c|c|c|c|c|c|}
\hline \multirow[b]{2}{*}{$\begin{array}{l}\text { Number of } \\
\text { threads per inch }\end{array}$} & \multirow[b]{2}{*}{$\begin{array}{c}\text { Pitch } \\
\text { diameter } \\
\text { tolerance, } \\
\text { pitch } \\
\text { increment }\end{array}$} & \multicolumn{3}{|c|}{ Serew } & \multicolumn{3}{|c|}{ Nut } \\
\hline & & $\begin{array}{l}\text { Tolerance } \\
\text { on major } \\
\text { diameter, } \\
\text { minus }\end{array}$ & $\begin{array}{l}\text { Maximum } \\
\text { minor } \\
\text { diameter = } \\
\text { maximum } \\
\text { major } \\
\text { diameter } \\
\text { minus }\end{array}$ & $\begin{array}{l}\text { Maximum } \\
\text { pitch } \\
\text { diameter }= \\
\text { maximum } \\
\text { major } \\
\text { diameter } \\
\text { minus }\end{array}$ & $\begin{array}{l}\text { Tolerance } \\
\text { on minor } \\
\text { diameter, } \\
\text { plus }\end{array}$ & $\begin{array}{c}\text { Minimum } \\
\text { minor } \\
\text { diameter }= \\
\text { basic } \\
\text { major } \\
\text { diameter } \\
\text { minus }\end{array}$ & $\begin{array}{l}\text { Minimum } \\
\text { pitch } \\
\text { diametcr }= \\
\text { minimum } \\
\text { major } \\
\text { diameter } \\
\text { minus }\end{array}$ \\
\hline 1 & 2 & 3 & 4 & 5 & 6 & $y$ & 8 \\
\hline $\begin{array}{l}64 \\
56 \\
48 \\
36\end{array}$ & $\begin{array}{l}\text { Inch } \\
0.00250 \\
.00267 \\
.00289 \\
.00316 \\
.00333\end{array}$ & $\begin{array}{l}\text { Inch } \\
0.0052 \\
.0056 \\
.0062 \\
.0068 \\
.0072\end{array}$ & $\begin{array}{r}\text { Inch } \\
0.0192 \\
.0219 \\
.0256 \\
.0307 \\
.0341\end{array}$ & $\begin{array}{l}\text { Inch } \\
0.0101 \\
.0116 \\
.0135 \\
.0162 \\
.0180\end{array}$ & $\begin{array}{l}\text { Inch } \\
0.0017 \\
.0019 \\
.0023 \\
.0027 \\
.0030\end{array}$ & $\begin{array}{l}\text { Inch } \\
0.0169 \\
.0193 \\
.0226 \\
.0271 \\
.0301\end{array}$ & $\begin{array}{r}\text { Inch } \\
0.0101 \\
.0116 \\
.0135 \\
.0162 \\
.0180\end{array}$ \\
\hline $\begin{array}{l}32 \\
28 \\
24 \\
20 \\
18\end{array}$ & $\begin{array}{l}.00354 \\
.00378 \\
.00408 \\
.00447 \\
.00471\end{array}$ & $\begin{array}{l}.0076 \\
.0086 \\
.0092 \\
.0102 \\
.0114\end{array}$ & $\begin{array}{l}.0383 \\
.0438 \\
.0511 \\
.0613 \\
.0682\end{array}$ & $\begin{array}{l}.0203 \\
.0232 \\
.0271 \\
.0325 \\
.0361\end{array}$ & $\begin{array}{l}.0034 \\
.0039 \\
.0045 \\
.0054 \\
.0060\end{array}$ & $\begin{array}{l}.0338 \\
.0387 \\
.0451 \\
.0541 \\
.0601\end{array}$ & $\begin{array}{l}.0203 \\
.0232 \\
.0271 \\
.0325 \\
.0361\end{array}$ \\
\hline $\begin{array}{r}16 \\
14 \\
10 \\
8\end{array}$ & $\begin{array}{l}.00500 \\
.00535 \\
.00577 \\
.00632 \\
.00707\end{array}$ & $\begin{array}{l}.0126 \\
.0140 \\
.0158 \\
.0184 \\
.0222\end{array}$ & $\begin{array}{l}.0767 \\
.0876 \\
.1022 \\
.1227 \\
.1534\end{array}$ & $\begin{array}{l}.0406 \\
.0464 \\
.0541 \\
.0650 \\
.0812\end{array}$ & $\begin{array}{l}.0068 \\
.0077 \\
.0090 \\
.0108 \\
.0135\end{array}$ & $\begin{array}{l}.0677 \\
.0773 \\
.0902 \\
.1083 \\
.1353\end{array}$ & $\begin{array}{l}.0406 \\
.0464 \\
.0541 \\
.0650 \\
.0812\end{array}$ \\
\hline $\begin{array}{l}5 \\
4 \\
4\end{array}$ & $\begin{array}{l}.00816 \\
.01000\end{array}$ & $\begin{array}{l}.0290 \\
.0408\end{array}$ & $\begin{array}{l}.2045 \\
.3067\end{array}$ & $\begin{array}{l}.1083 \\
.1624\end{array}$ & $\begin{array}{l}.0180 \\
.0271\end{array}$ & $\begin{array}{l}.1804 \\
.2706\end{array}$ & $\begin{array}{l}.1083 \\
.1624\end{array}$ \\
\hline
\end{tabular}

TABLE 40.-Class B, diameter increments of pitch diameter tolerances

\begin{tabular}{|c|c|}
\hline Diameter & $\begin{array}{c}\text { Diameter } \\
\text { increment }\end{array}$ \\
\cline { 1 - 2 } Inches & 2 \\
\cline { 1 - 2 } .0625 & Inch \\
.125 & 0.00050 \\
.1875 & .00071 \\
.250 & .00087 \\
.375 & .00100 \\
& .00122 \\
.500 & .00141 \\
.750 & .00173 \\
1.000 & .00200 \\
1.500 & .00245 \\
2.000 & .00283 \\
& \\
3.000 & .00346 \\
4.000 & .00400 \\
6.000 & .00490 \\
8.000 & .00566 \\
12.000 & .00693 \\
& \\
&
\end{tabular}

TABLE 41.-Class $B$, length of engagement increments of pitch diameter tolerances

\begin{tabular}{|c|c|}
\hline $\begin{array}{c}\text { Length of } \\
\text { engagement }\end{array}$ & Increment \\
\hline 1 & 2 \\
\hline Inches & Inch \\
0.250 & 0.00050 \\
.500 & .00100 \\
1.000 & .00200 \\
2.000 & .00400 \\
3.000 & .00600 \\
4.000 & .00800 \\
\hline
\end{tabular}


3. Class C.-This class is intended to cover the manufacture of threaded parts which are to assemble nearly, or entirely, with the fingers, and where a slight amount of shake or play between the assembled threaded members is not objectionable. It is the same in every particular as class B except that the tolerances are smaller.

The pitch diameter tolerances are obtained by adding three increments, as follows:

$$
\begin{array}{ll}
\text { Diameter increment } & =0.002 \sqrt{D} \\
\text { 'Length of engagement increment } & =0.002 Q \\
\text { Pitch increment } & =0.010 \sqrt{p}
\end{array}
$$

where

$$
\begin{aligned}
& D=\text { basic major diameter } \\
& Q=\text { length of engagement } \\
& p=\text { pitch of thread in inches. }
\end{aligned}
$$

Tables 42,43 , and 44 give the tolerances for major and minor diameters, and tolerance increments for pitch diameters for a range of diameters, lengths of engagements, and pitches. The total pitch diameter tolerance may be obtained by adding increments obtained from the tables, or it may be obtained directly from the chart, Figure 23.

Example: 4-inch 24-thread, class C, three-eighths inch length of engagement.

From Table 42 we get for the screw:

Maximum major diameter........... $=4.000$

Minimum major diameter $=4.000-0.0066=3.9934$

Maximum minor diameter $=4.000-.0511=3.9489$

Maximum pitch diameter $=4.000-.0271=3.9729$

And for the nut:

Minimum major diameter

Minimum minor diameter $=4.000-.0451=3.9549$

Maximum minor diameter $=3.9549+.0045=3.9594$

Minimum pitch diameter $=4.000-.0271=3.9729$

Also for the pitch diameter tolerance:

Pitch increment. . .

From Table 43, diameter increment ( 4 inches) - $=.00400$

From Table 44, engagement increment $(3 / 8$

inch) . . .

Total

Increment values are given to five decimal places for purposes of calculation. The final result may be rounded off to the nearest third or fourth decimal place. The pitch diameter tolerance in this example for both screw and nut can be 0.0068 , or 0.007 inch.

The pitch diameter tolerance should never be appreciably greater than the tolerance on the major diameter of the screw. If the cal- . culated pitch diameter tolerance exceeds the tolerance on the major 
diameter of the screw, it is evidence that too fine a pitch or too loose a fit has been selected. In the foregoing example, the pitch diameter tolerance is slightly greater than the tolerance on the major diameter of the screw. This example is a limiting case.

TABLE 42.-Class $C$, pitch increments

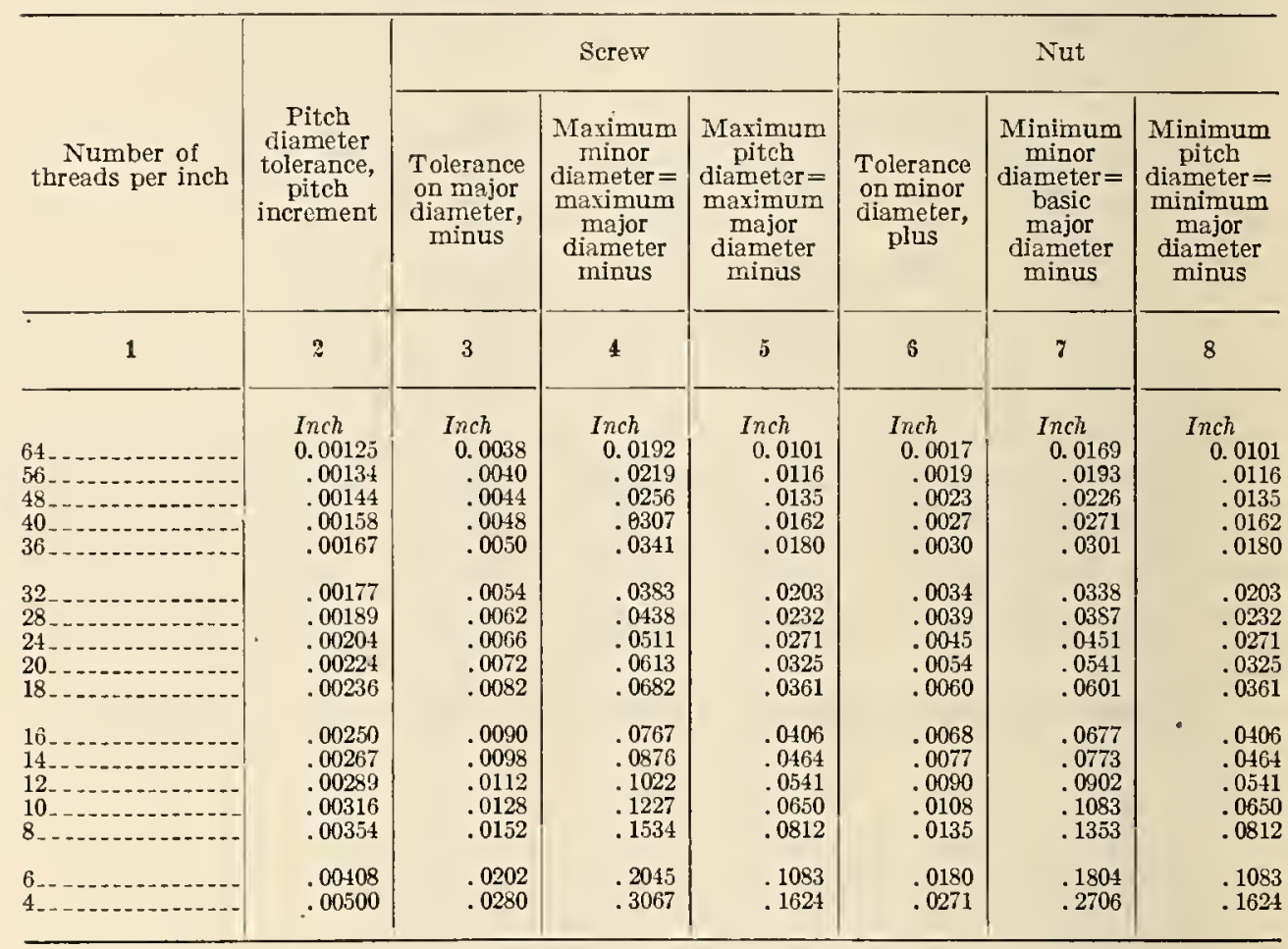

TABLe 43.-Class $C$, diameter increments of pitch diameter tolerances

\begin{tabular}{|c|c|}
\hline Diameter & $\begin{array}{c}\text { Diameter } \\
\text { increment }\end{array}$ \\
\hline 1 & 2 \\
\hline Inches &. Inch \\
0.0625 & 0.00050 \\
.125 & .00071 \\
.1875 & .00087 \\
.250 & .00100 \\
.375 & .00122 \\
.500 & .00141 \\
.750 & .00173 \\
1.000 & .00200 \\
1.500 & .00245 \\
2.000 & .00283 \\
3.000 & .00346 \\
4.000 & .00100 \\
6.000 & .00490 \\
8.000 & .00566 \\
12.000 & .00693 \\
\hline
\end{tabular}


$\mathrm{T}_{\mathrm{ABLE}}$ 44.-Class $C$, length of engagement increments of pitch diameter tolerances

\begin{tabular}{|c|c|}
\hline $\begin{array}{c}\text { Length of } \\
\text { engagement }\end{array}$ & Increment \\
\cline { 2 - 2 } & 2 \\
\hline 1 & Inch \\
Inches & 0.00050 \\
0.250 & .00100 \\
.500 & .00200 \\
1.000 & .00400 \\
2.000 & .00600 \\
3.000 & .00800 \\
4.000 & \\
\hline
\end{tabular}

4. Class D.-This class is intended to cover the manufacture of the higher grade of threaded parts which are to assemble nearly or entirely with the fingers and must have the minimum amount of shake or play between the threaded members. It is the same as class $\mathrm{C}$ in every particular except that the tolerances are smaller.

The pitch diameter tolerances are obtained by adding three increments, as follows:

$$
\begin{array}{ll}
\text { Diameter increment } & =0.002 \sqrt{D} \\
\text { Length of engagement increment } & =.002 Q \\
\text { Pitch increment } & =.005 \sqrt{p}
\end{array}
$$

where

$$
\begin{aligned}
& D=\text { basic major diameter } \\
& Q=\text { length of engagement } \\
& p=\text { pitch of thread in inches. }
\end{aligned}
$$

Tables 45, 46, and 47 give the tolerances for major and minor diameters, and tolerance increments for pitch diameters for a range of diameters, lengths of engagements, and pitches. The total pitch diameter tolerance may be obtained by adding increments obtained from the tables, or it may be obtained directly from the chart, Figure 23.

Example: 31/2-inch, 20-thread, class D, five-eighths inch length of engagement.

From Table 45 we get for the screw:

Maximum major diameter $\quad=3.500$

Minimum major diameter $=3.500-0.0072=3.4928$

Maximum minor diameter $=3.500-.0613=3.4387$

Maximum pitch diameter $=3.500-.0325=3.4675$

And for the nut:

Minimum major diameter $\quad=3.500$

Minimum minor diameter $=3.500-.0541=3.4459$

Maximum minor diameter $=3.4459+.0054=3.4513$

Minimum pitch diameter $=3.500-.0325=3.4675$

Also for the pitch diameter tolerance:

Pitch increment

From Table 46 , diameter increment $(31 / 2$ inches $)=.00373$

From Table 47 , engagement increment $(5 / 8 \mathrm{inch})=.00125\}$ (by interpolation)

Total .00610 
Increment values are given to five decimal places for purposes of calculation. The final result may be rounded off to the nearest third or fourth decimal place. The pitch diameter tolerance in this example for both screw and nut can be 0.0061 , or 0.006 inch.

The pitch diameter tolerance should never be appreciably greater than the tolerance on the major diameter of the screw. If the calculated pitch diameter tolerance exceeds the tolerance on the major diameter of the screw, it is evidence that too fine a pitch or too loose a fit has been selected.

TABLE 45.-Class D, pitch increments

\begin{tabular}{|c|c|c|c|c|c|c|c|}
\hline \multirow[b]{2}{*}{$\begin{array}{c}\text { Number of } \\
\text { threads per inch }\end{array}$} & \multirow[b]{2}{*}{$\begin{array}{c}\text { Pitch } \\
\text { diameter } \\
\text { tolerance, } \\
\text { pitch } \\
\text { increment } \\
\end{array}$} & \multicolumn{3}{|c|}{ Screw } & \multicolumn{3}{|c|}{ Nut } \\
\hline & & $\begin{array}{l}\text { Tolerance } \\
\text { on major } \\
\text { diameter } \\
\text { minus }\end{array}$ & $\begin{array}{l}\text { Marimum } \\
\text { minor } \\
\text { diameter = } \\
\text { maximum } \\
\text { major } \\
\text { diameter } \\
\text { minus }\end{array}$ & $\begin{array}{l}\text { Maximum } \\
\text { pitch } \\
\text { diameter = } \\
\text { maximum } \\
\text { major } \\
\text { diameter } \\
\text { minus }\end{array}$ & $\begin{array}{c}\text { Tolerance } \\
\text { on minor } \\
\text { diameter, } \\
\text { plus }\end{array}$ & $\begin{array}{l}\text { Minimum } \\
\text { mizor } \\
\text { diameter }= \\
\text { basic } \\
\text { major } \\
\text { diameter } \\
\text { minus }\end{array}$ & $\begin{array}{l}\text { Minimum } \\
\text { pitch } \\
\text { diameter = } \\
\text { minimum } \\
\text { major } \\
\text { diameter } \\
\text { minus }\end{array}$ \\
\hline 1 & 2 & 3 & 4 & 5 & 6 & $\gamma$ & 8 \\
\hline $\begin{array}{l}64 \ldots \ldots \\
56 \ldots \ldots \\
48 \ldots \ldots \\
40 \ldots \ldots \\
36 \ldots \ldots\end{array}$ & $\begin{array}{l}\text { Inch } \\
0.00062 \\
.00067 \\
.00072 \\
.00079 \\
.00083\end{array}$ & $\begin{array}{l}\text { Inch } \\
0.0038 \\
.0040 \\
.0044 \\
.0048 \\
.0050\end{array}$ & $\begin{array}{l}\text { Inch } \\
0.0192 \\
.0219 \\
.0256 \\
.0307 \\
.0341\end{array}$ & $\begin{array}{l}\text { Inch } \\
0.0101 \\
.0116 \\
.0135 \\
.0162 \\
.0180\end{array}$ & $\begin{array}{l}\text { Inch } \\
0.0017 \\
.0019 \\
.0023 \\
.0027 \\
0030\end{array}$ & $\begin{array}{l}\text { Inch } \\
0.0169 \\
.0193 \\
.0226 \\
.0271 \\
.0301\end{array}$ & $\begin{array}{l}\text { Inch } \\
0.0101 \\
.0116 \\
.0135 \\
.0162 \\
.0180\end{array}$ \\
\hline $\begin{array}{l}32 \\
28 \\
24 \\
20 \\
18\end{array}$ & $\begin{array}{l}.00088 \\
.00094 \\
.00102 \\
.00112 \\
.00118\end{array}$ & $\begin{array}{l}.0054 \\
.0062 \\
.0066 \\
.0072 \\
.0082\end{array}$ & $\begin{array}{l}.0383 \\
.0438 \\
.0511 \\
.0613 \\
.0682\end{array}$ & $\begin{array}{l}.0203 \\
.0232 \\
.0271 \\
.0325 \\
.0361\end{array}$ & $\begin{array}{l}.0034 \\
.0039 \\
.0045 \\
.0054 \\
.0060\end{array}$ & $\begin{array}{l}.0338 \\
.0387 \\
0451 \\
.0541 \\
.0601\end{array}$ & $\begin{array}{l}.0203 \\
.0232 \\
.0271 \\
.0325 \\
.0361\end{array}$ \\
\hline $\begin{array}{l}16 \\
14 \\
12 \\
10\end{array}$ & $\begin{array}{l}.00125 \\
.00134 \\
.00144 \\
.00158 \\
.00177\end{array}$ & $\begin{array}{l}.0090 \\
.0098 \\
.0112 \\
.0125 \\
.0152\end{array}$ & $\begin{array}{l}.0767 \\
.0876 \\
.1022 \\
.1227 \\
.1534\end{array}$ & $\begin{array}{l}.0406 \\
.0464 \\
.0541 \\
.0650 \\
.0812\end{array}$ & $\begin{array}{l}.0068 \\
.0077 \\
.0090 \\
.0108 \\
.0135\end{array}$ & $\begin{array}{l}.0677 \\
.0773 \\
.0902 \\
.1083 \\
.1353\end{array}$ & $\begin{array}{l}.0405 \\
.0464 \\
.0541 \\
.0650 \\
.0812\end{array}$ \\
\hline 6 & $\begin{array}{l}.00204 \\
.00250\end{array}$ & $\begin{array}{l}.0202 \\
.0280\end{array}$ & $\begin{array}{l}.2045 \\
.3067\end{array}$ & $\begin{array}{l}.1083 \\
.1624\end{array}$ & $\begin{array}{l}.0180 \\
.0271\end{array}$ & .1804 & $\begin{array}{l}.1083 \\
.1624\end{array}$ \\
\hline
\end{tabular}

TABLE 46.-Class $D$, diameter increments of pitch diameter tolerances

\begin{tabular}{|c|c|}
\hline Diameter & $\begin{array}{c}\text { Diameter } \\
\text { increment }\end{array}$ \\
\hline 1 & 2 \\
\hline Inches & Inch \\
0.0625 & 0.00050 \\
.125 & .00071 \\
.1875 & .00087 \\
.250 & .00100 \\
.375 & .00122 \\
.500 & .00141 \\
.750 & .00173 \\
1.000 & .00200 \\
1.500 & .00245 \\
2.000 & .00283 \\
3.000 & .00346 \\
4.000 & .00400 \\
6.000 & .00490 \\
8.000 & .00566 \\
12.000 & .00693 \\
\hline
\end{tabular}


TABLE 47.-Class $D$, length of engagement increments of pitch diameter tolerances

\begin{tabular}{|c|c|}
\hline $\begin{array}{c}\text { Length of } \\
\text { engagement }\end{array}$ & Increment \\
\hline 1 & 2 \\
\hline Inches & Inch \\
0.250 & 0.00050 \\
1.500 & .00000 \\
1.000 & .00200 \\
3.000 & .00400 \\
3.000 & .00600 \\
4.000 & .00800 \\
\hline
\end{tabular}

5. Class E.-This class is intended to cover the manufacture of threaded parts of the finest commercial quality, where very little shake or play is desirable, and where a screwdriver or wrench may be necessary for assembly.

In the manufacture of screw-thread products belonging to this class it may be necessary to use precision tools, selected gages, and other refinements. This quality of work should, therefore, be used in cases only where requirements of the mechanism being produced are exacting. In order to secure the fit desired, it may be necessary in some cases to select the parts when the product is being assembled.

The maximum pitch diameters of the screws are slightly larger than the minimum pitch diameters of the nuts as determined from Table 48.

The pitch diameter tolerances are obtained by adding three increments, as follows:

Diameter increment $\quad=0.001 \sqrt{D}$

Length of engagement increment $=.001 \quad Q$

where$$
\text { Pitch increment } \quad=.0025 \sqrt{p}
$$

$$
\begin{aligned}
& D=\text { basic major diameter } \\
& Q=\text { length of engagement } \\
& p=\text { pitch of thread in inches. }
\end{aligned}
$$

Tables 48,49 , and 50 give the tolerances for major diameter and minor diameter, and tolerance increments for pitch diameter for a range of diameters, lengths of engagement, and pitches. The total pitch diameter tolerance may be obtained by adding increments obtained from the tables, or it may be obtained directly from the chart, Figure 23. 
Example: $41 / 4$-inch, 16 thread, class $\mathrm{E}$, three-fourths inch length of engagement.

From Table 48 we get for the screw:

Maximum major diameter $=4.250$

Minimum major diameter $=4.250-0.0090=4.2410$

Maximum minor diameter $=4.250-.0767=4.1733$

Maximum pitch diameter $=4.250-.0402=4.2098$ And for the nut:

Minimum major diameter $\quad=4.250$

Minimum minor diameter $=4.250-0.0677=4.1823$

Maximum minor diameter $=4.1823+.0068=4.1891$

Minimum pitch diameter $=4.250-.0406=4.2094$

Also for the pitch diameter tolerance:

Pitch increment................... $=.00062$

$\left.\begin{array}{l}\text { From Table } 49 \text {, diameter increment }(41 / 4 \text { inches })=.00206 \\ \text { From Table 50, engagement increment }(3 / 4 \text { inch })-=.00075\end{array}\right\}$ By interpolation.

Total

Increment values are given to five decimal places for purposes of calculation. The final result may be rounded off to the nearest fourth decimal place. The pitch diameter tolerance in this example for both screw and nut would be 0.0034 inch.

The pitch diameter tolerance should never be appreciably greater than the tolerance on the major diameter of the screw. If the calculated pitch diameter tolerance exceeds the tolerance on the major diameter of the screw, it is evidence that too fine a pitch has been selected.

TABLE 48.-Class $E$, pitch increments

\begin{tabular}{|c|c|c|c|c|c|c|c|}
\hline \multirow[b]{2}{*}{$\begin{array}{l}\text { Number of } \\
\text { threads per inch }\end{array}$} & \multirow[b]{2}{*}{$\begin{array}{l}\text { Pitch } \\
\text { diameter } \\
\text { tolerance, } \\
\text { pitch } \\
\text { increment }\end{array}$} & \multicolumn{3}{|c|}{ Screw } & \multicolumn{3}{|c|}{ Nut } \\
\hline & & $\begin{array}{l}\text { Tolerance } \\
\text { on major } \\
\text { diameter, } \\
\text { minus }\end{array}$ & $\begin{array}{c}\text { Maximum } \\
\text { minor } \\
\text { diameter }= \\
\text { maximum } \\
\text { major } \\
\text { diameter } \\
\text { minus }\end{array}$ & $\begin{array}{c}\text { Maximum } \\
\text { pitch } \\
\text { diameter = } \\
\text { maximum } \\
\text { major } \\
\text { diameter } \\
\text { minus }\end{array}$ & $\begin{array}{l}\text { Tolerance } \\
\text { on minor } \\
\text { diameter, } \\
\text { plus }\end{array}$ & $\begin{array}{c}\text { Minimum } \\
\text { minor } \\
\text { diameter }= \\
\text { basic } \\
\text { major } \\
\text { diameter } \\
\text { minus }\end{array}$ & $\begin{array}{l}\text { Minimum } \\
\text { pitch } \\
\text { diameter= } \\
\text { minimum } \\
\text { major } \\
\text { diameter } \\
\text { minus }\end{array}$ \\
\hline 1 & 2 & 3 & 4 & 5 & 6 & 7 & 8 \\
\hline $\begin{array}{l}64 \ldots \\
56-\ldots \\
48 \\
40 \ldots \\
36 \ldots\end{array}$ & $\begin{array}{l}\text { Inch } \\
0.00031 \\
.00033 \\
.00036 \\
.00040 \\
.00042\end{array}$ & $\begin{array}{c}\text { Inch } \\
0.0038 \\
.0040 \\
.0044 \\
.0048 \\
.0050\end{array}$ & $\begin{array}{l}\text { Inch } \\
0.0192 \\
.0219 \\
.0256 \\
.0307 \\
.0341\end{array}$ & $\begin{array}{c}\text { Inch } \\
0.0100 \\
.0114 \\
.0133 \\
.0160 \\
.0178\end{array}$ & $\begin{array}{l}\text { Inch } \\
0.0017 \\
.0019 \\
.0023 \\
.0027 \\
.0030\end{array}$ & $\begin{array}{c}\text { Inch } \\
0.0169 \\
.0193 \\
.0226 \\
.0271 \\
.0301\end{array}$ & $\begin{array}{l}\text { Inch } \\
0.0101 \\
0.0116 \\
.0135 \\
.0162 \\
.0180\end{array}$ \\
\hline $\begin{array}{l}32 \\
28 \\
24 \\
20 \\
18\end{array}$ & $\begin{array}{l}.00044 \\
.00047 \\
.00051 \\
.00056 \\
.00059\end{array}$ & $\begin{array}{l}.0054 \\
.0062 \\
.0066 \\
.0072 \\
.0082\end{array}$ & $\begin{array}{l}.0383 \\
.0438 \\
.0511 \\
.0613 \\
.0682\end{array}$ & $\begin{array}{l}.0201 \\
.0230 \\
.0268 \\
.0322 \\
.0358\end{array}$ & $\begin{array}{l}.0034 \\
.0039 \\
.0045 \\
.0054 \\
.0060\end{array}$ & $\begin{array}{l}.0338 \\
.0387 \\
.0451 \\
.0541 \\
.0601\end{array}$ & $\begin{array}{l}.0203 \\
.0232 \\
.0271 \\
.0325 \\
.0361\end{array}$ \\
\hline $\begin{array}{r}16 \\
14 \\
10 \\
10 \\
8\end{array}$ & $\begin{array}{l}.00062 \\
.00067 \\
.00072 \\
.00079 \\
.00088\end{array}$ & $\begin{array}{l}.0090 \\
.0098 \\
.0112 \\
.0128 \\
.0152\end{array}$ & $\begin{array}{l}.0767 \\
.0876 \\
.1022 \\
.1227 \\
.1534\end{array}$ & $\begin{array}{l}.0402 \\
.0460 \\
.0536 \\
.0644 \\
.0805\end{array}$ & $\begin{array}{l}.0068 \\
.0077 \\
.0090 \\
.0108 \\
.0135\end{array}$ & $\begin{array}{l}.0677 \\
.0773 \\
.0902 \\
.1083 \\
.1353\end{array}$ & $\begin{array}{l}.0406 \\
.0464 \\
.0541 \\
.0650 \\
.0812\end{array}$ \\
\hline $\begin{array}{l}6 \ldots \\
4 \ldots\end{array}$ & $\begin{array}{l}.00102 \\
.00125\end{array}$ & $\begin{array}{l}.0202 \\
.0280\end{array}$ & $\begin{array}{l}.2045 \\
.3067\end{array}$ & $\begin{array}{l}.1074 \\
.1611\end{array}$ & $\begin{array}{l}.0180 \\
.0271\end{array}$ & $\begin{array}{l}.1804 \\
.2706\end{array}$ & $\begin{array}{l}.1083 \\
.1624\end{array}$ \\
\hline
\end{tabular}


TABLE 49.-Class E, diameter increments of pitch diameter tolerances

\begin{tabular}{|c|c|}
\hline Diameter & $\begin{array}{c}\text { Diameter } \\
\text { increment }\end{array}$ \\
\hline 1 & 2 \\
\hline Inches & Inch \\
0.0625 & 0.00025 \\
.125 & .00035 \\
.1875 & .00043 \\
.250 & .00050 \\
.375 & .00061 \\
.500 & .00071 \\
.750 & .00087 \\
1.000 & .00100 \\
1.500 & .00122 \\
2.000 & .00141 \\
3.000 & .00173 \\
4.000 & .00200 \\
6.000 & .00245 \\
8.000 & .00283 \\
12.000 & .00346 \\
& \\
\hline
\end{tabular}

TABLE 50.-Class E, length of engagement increments of pitch diameter tolerances

\begin{tabular}{|c|c|}
\hline $\begin{array}{c}\text { Length of } \\
\text { engagement }\end{array}$ & Increment \\
\cline { 1 - 2 } & \\
\hline 1 & \\
\hline Inches & Inch \\
0.250 & 0.00025 \\
.500 & .00050 \\
1.000 & .00100 \\
2.000 & .00200 \\
3.000 & .00300 \\
4.000 & .00400 \\
\hline
\end{tabular}

\section{SPECIFICATIONS FOR THREADING TOOLS}

(a) FORM OF TOOLS FOR PRODUCING SCREWS

The specifications given in Section III, division 5(a), for tolerances on chasers, thread milling cutters, roller dies, threading hobs, etc., are also applicable to screw threads of special diameter and pitches.

(b) TAPS

1. Tolerances FOR TAPs.-Tables of tolerances for three classes of taps are given: First, commercial taps; second, Class $\mathrm{X}$ ground taps; and third, class $Y$ ground taps. (See Tables 51, 52, and 53.)

The selection of the tap will depend upon the tolerances on the tapped hole. A tap should be selected whose tolerance does not exceed 75 per cent of the tolerance on the tapped hole. 
2. Marking of Taps.-Taps shall be marked indicating the diameter, pitch, and thread form as follows (see Section II, division 2, "Symbols") :

A 1-inch tap, 16 threads per inch, national form of thread, will be marked $1^{\prime \prime}-16-N$.

A 1-inch tap, 16 threads per inch, left hand, national form of thread, will be marked: $1^{\prime \prime}-16$ L. H. $-N$.

A multiple threaded tap will be marked as above, and also with the number of starts. Where the size of the shank is large enough, the lead should also be given.

A double-threaded tap; 1 inch diameter, 16 threads per inch, national form of thread, with a lead of 0.125 inch will be marked: $1^{\prime \prime}-16-N$ Double, Lead-0.125.

3. Shape of Cutting Edge for Taps.-The specifications given in Section III, division 5(b), for shape of cutting edge of taps and other internal threading tools are also applicable to screw threads of special diameters and pitches.

\section{(c) TAP DRILLS}

The essential requirement of a tap drill is that the hole produced by it shall be such that, after tapping, the minor diameter of the tapped hole shall be within the specified limits. For tapped holes of special diameters and pitches, the standard drill that comes nearest in sizé to the mean of the maximum and minimum minor diameters of the tapped hole should be selected, if the diameter is within the specified limits.

TABLE 51.-Tolerances for commercial taps, special diameters and pitches

\begin{tabular}{|c|c|c|c|c|c|c|c|c|}
\hline \multirow{2}{*}{\multicolumn{2}{|c|}{ Sizes }} & \multirow{3}{*}{$\begin{array}{c}\text { Major } \\
\text { diameter } \\
\begin{array}{c}\text { Minimum } \\
=\text { basic } \\
\text { major diam- } \\
\text { eter plus }\end{array}\end{array}$} & \multicolumn{6}{|c|}{ Pitch diameter } \\
\hline & & & \multicolumn{3}{|c|}{$\begin{array}{l}\text { Coarser pitchcs than "national } \\
\text { fine-thread series" }\end{array}$} & \multicolumn{3}{|c|}{$\begin{array}{l}\text { Finer pitches than "national } \\
\text { fine-thread series" }\end{array}$} \\
\hline From- & To- & & $\begin{array}{c}\text { Minimum } \\
=\text { minimum } \\
\text { pitch diam- } \\
\text { eter of } \\
\text { hole plus }\end{array}$ & $\begin{array}{l}\text { Tolerance, } \\
\text { plus }\end{array}$ & $\begin{array}{l}\text { Masimum } \\
=\text { minimum } \\
\text { pitch diam- } \\
\text { eter of } \\
\text { hole plus }\end{array}$ & $\begin{array}{c}\text { Minimum } \\
=\text { minimum } \\
\text { pitch diam- } \\
\text { eter of } \\
\text { hole plus }\end{array}$ & $\begin{array}{l}\text { Tolerance, } \\
\text { plus }\end{array}$ & $\begin{array}{l}\text { Maximum } \\
=\text { minimum } \\
\text { pitch diam } \\
\text { eter of } \\
\text { hole plus }\end{array}$ \\
\hline \multicolumn{2}{|l|}{1} & 2 & 3 & 4 & 5 & 6 & 7 & 8 \\
\hline $\begin{array}{r}1 / 4 \\
77 \\
36 \\
11 \\
118 \\
15 \\
218 \\
258 \\
31 / 8\end{array}$ & $\begin{array}{l}3 / 8 \\
5 / 8 \\
1^{5} \\
11 / 2 \\
2 \\
21 / 2 \\
3 \\
4\end{array}$ & $\begin{array}{l}\text { Inch } \\
0.0010 \\
.0010 \\
.0010 \\
.0015 \\
.0015 \\
.0015 \\
.0015 \\
.0015\end{array}$ & $\begin{array}{l}\text { Inch } \\
0.0005 \\
.0005 \\
.0005 \\
.0005 \\
.0010 \\
.0010 \\
.0010 \\
.0010\end{array}$ & $\begin{array}{l}\text { Inch } \\
0.0020 \\
.0025 \\
.0030 \\
.0035 \\
.0040 \\
.0045 \\
.0050 \\
.0055\end{array}$ & $\begin{array}{l}\text { Inch } \\
0.0025 \\
.0030 \\
.0035 \\
.0040 \\
.0050 \\
.0055 \\
.0060 \\
.0065\end{array}$ & $\begin{array}{l}\text { Inch } \\
0.0005 \\
.0005 \\
.0005 \\
.0005 \\
.0005 \\
.0005 \\
.0005 \\
.0005\end{array}$ & $\begin{array}{l}\text { Inch } \\
0.0015 \\
.0020 \\
.0025 \\
.0030 \\
.0030 \\
.0035 \\
.0035 \\
.0045\end{array}$ & $\begin{array}{l}\text { Inch } \\
0.0020 \\
.0025 \\
.0030 \\
.0035 \\
.0035 \\
.0040 \\
.0040 \\
.0050\end{array}$ \\
\hline
\end{tabular}

Nore.-Marimum lead error $= \pm 0.003$ inch in 1 inch of thread. 
TABLE 52.-Tolerances for class $X$ ground taps, special diameters and pitches

\begin{tabular}{|c|c|c|c|c|c|c|c|c|}
\hline \multirow{2}{*}{\multicolumn{2}{|c|}{ Sizes }} & \multirow{3}{*}{$\mid \begin{array}{c}\text { Major } \\
\text { diameter }\end{array}$} & \multicolumn{6}{|c|}{ Pitch diameter } \\
\hline & & & \multicolumn{3}{|c|}{$\begin{array}{l}\text { Coarser pitches than "national } \\
\text { fne-thread series" }\end{array}$} & \multicolumn{3}{|c|}{$\begin{array}{l}\text { Finer pitches than "national } \\
\text { fne-thread series" }\end{array}$} \\
\hline From- & To- & & $\begin{array}{c}\text { Minimum } \\
=\text { minimum } \\
\text { pitch diam- } \\
\text { eter of } \\
\text { hole plus }\end{array}$ & $\begin{array}{c}\text { Tolerance, } \\
\text { plus }\end{array}$ & $\begin{array}{l}\text { Maximum } \\
=\text { minimum } \\
\text { pitch diam- } \\
\text { eter of } \\
\text { hole plus }\end{array}$ & $\begin{array}{l}\text { Minimum } \\
=\text { minimum } \\
\text { pitch diam- } \\
\text { eter of } \\
\text { hole plus }\end{array}$ & $\begin{array}{c}\text { Tolerance, } \\
\text { plus }\end{array}$ & $\begin{array}{c}\text { Maximum } \\
=\text { minimum } \\
\text { pitch diam- } \\
\text { eter of } \\
\text { hole plus }\end{array}$ \\
\hline \multicolumn{2}{|l|}{1} & 2 & 3 & 4 & 5 & 6 & 7 & 8 \\
\hline 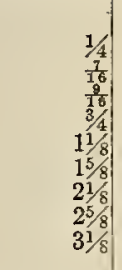 & $\begin{array}{l}3 / 8 \\
1 / 2 \\
5 / 8 \\
1 \\
11 / 2 \\
2 \\
21 / 2 \\
3 \\
4\end{array}$ & $\begin{array}{c}\text { Inch } \\
0.0020 \\
.0025 \\
.0025 \\
.0030 \\
.0040 \\
.0050 \\
.0050 \\
.0050 \\
.0050\end{array}$ & $\begin{array}{c}\text { Inch } \\
0.0000 \\
.0000 \\
.0000 \\
.0000 \\
.0000 \\
.0000 \\
.0000 \\
.0000 \\
.0000\end{array}$ & $\begin{array}{l}\text { Inch } \\
0.0008 \\
.0009 \\
.0010 \\
.0012 \\
.0015 \\
.0015 \\
.0015 \\
.0015 \\
.0015\end{array}$ & $\begin{array}{l}\text { Inch } \\
0.0008 \\
.0009 \\
.0010 \\
.0012 \\
.0015 \\
.0015 \\
.0015 \\
.0015 \\
.0015\end{array}$ & $\begin{array}{r}\text { Inch } \\
0.0000 \\
.0000 \\
.0000 \\
.0000 \\
.0000 \\
.0000 \\
.0000 \\
.0000 \\
.0000\end{array}$ & $\begin{array}{c}\text { Inch } \\
0.0008 \\
.0008 \\
.0008 \\
.0009 \\
.0010 \\
.0015 \\
.0015 \\
.0015 \\
.0015\end{array}$ & $\begin{array}{r}\text { Inch } \\
0.0008 \\
.0008 \\
.0008 \\
.0009 \\
.0010 \\
.0015 \\
.0015 \\
.0015 \\
.0015\end{array}$ \\
\hline
\end{tabular}

NOTE. - Maximum lead error $= \pm 0.0005$ inch in 1 inch of thread. Tolerance on half angle of thread $= \pm 15$ minutes

TABLE 53.-Tolerances for class $Y$ ground taps, special diameters and pitches

\begin{tabular}{|c|c|c|c|c|c|c|c|c|}
\hline \multirow{2}{*}{\multicolumn{2}{|c|}{ Sizes }} & \multirow{3}{*}{$\begin{array}{c}\begin{array}{c}\text { Major } \\
\text { diameter }\end{array} \\
\begin{array}{c}\text { Minimum } \\
\text { =basic } \\
\text { major diam- } \\
\text { eter plus }\end{array}\end{array}$} & \multicolumn{6}{|c|}{ Pitch diameter } \\
\hline & & & \multicolumn{3}{|c|}{$\begin{array}{l}\text { Coarser pitches than "national } \\
\text { fine-thread series" }\end{array}$} & \multicolumn{3}{|c|}{$\begin{array}{l}\text { Finer pitches than "national } \\
\text { fine-thread series" }\end{array}$} \\
\hline From- & To- & & $\begin{array}{c}\text { Minimum } \\
=\text { minimum } \\
\text { pitch diam- } \\
\text { eter of } \\
\text { hole plus }\end{array}$ & $\begin{array}{l}\text { Tolerance, } \\
\text { plus }\end{array}$ & $\begin{array}{l}\text { Maximum } \\
\text { =minimum } \\
\text { pitch diam- } \\
\text { mcter of } \\
\text { hole plus }\end{array}$ & $\begin{array}{l}\text { Minimum } \\
=\text { minimum } \\
\text { pitch diam- } \\
\text { eter of } \\
\text { hole plus }\end{array}$ & $\begin{array}{c}\text { Tolerance, } \\
\text { plus }\end{array}$ & $\begin{array}{l}\text { Maximum } \\
=\text { minimum } \\
\text { pitch diam- } \\
\text { eter of } \\
\text { hole plus }\end{array}$ \\
\hline \multicolumn{2}{|l|}{1} & 2 & 3 & 4 & 5 & 6 & 7 & 8 \\
\hline \begin{tabular}{r|}
$1 / 4$ \\
7 \\
18 \\
$\frac{8}{86}$ \\
$8 / 4$ \\
$11 / 8$ \\
$15 / 8$ \\
$21 / 8$ \\
$25 / 8$ \\
$31 / 8$
\end{tabular} & $\begin{array}{l}3 / 8 \\
1 / 2 \\
5 / 8 \\
1^{1 / 2} \\
11 / 2 \\
2 \\
21 / 2 \\
3 \\
4\end{array}$ & $\begin{array}{l}\text { Inch } \\
0.0025 \\
.0030 \\
.0035 \\
.0040 \\
.0050 \\
.0065 \\
.0080 \\
.0080 \\
.0080\end{array}$ & $\begin{array}{c}\text { Inch } \\
0.0006 \\
.0007 \\
.0008 \\
.0010 \\
.0012 \\
.0015 \\
.0020 \\
.0020 \\
.0020\end{array}$ & $\begin{array}{l}\text { Inch } \\
0.0010 \\
.0011 \\
.0013 \\
.0015 \\
.0020 \\
.0025 \\
.0030 \\
.0030 \\
.0030\end{array}$ & $\begin{array}{l}\text { Inch } \\
0.0016 \\
.0018 \\
.0021 \\
.0025 \\
.0032 \\
.0040 \\
.0050 \\
.0050 \\
.0050\end{array}$ & $\begin{array}{l}\text { Inch } \\
0.0005 \\
.0005 \\
.0006 \\
.0007 \\
.0008 \\
.0010 \\
.0010 \\
.0015 \\
.0015\end{array}$ & $\begin{array}{c}\text { Inch } \\
0.0010 \\
.0010 \\
.0010 \\
.0013 \\
.0015 \\
.0018 \\
.0020 \\
.0025 \\
.0025\end{array}$ & $\begin{array}{l}\text { Inch } \\
0.0015 \\
.0015 \\
.0016 \\
.0020 \\
.0023 \\
.0028 \\
.0030 \\
.0040 \\
.0040\end{array}$ \\
\hline
\end{tabular}

Nоте.-Maximum lead error $= \pm 0.0005$ inch in 1 inch of thread. Tolerance on half angle of thread $=$ \pm 20 minutes.

\section{GAGES}

(See also Section III, division 5, "gages")

(a) SPECIFICATIONS FOR GAGES

1. Classification of Gages, and Gage Tolerances.-Screwthread gages are classified according to accuracy into classes $\mathrm{X}, \mathrm{Y}$, and $\mathrm{Z}$, the class $\mathrm{X}$ being the most accurate. The tolerance limits on classes $\mathrm{Y}$ and $\mathrm{Z}$ "go" gages are placed inside of the extreme product 
limits to provide allowance for wear of the gages. The tolerances on all "not go" gages, however, are applied from the extreme product limit as the starting point, as no allowance for wear is necessary. Also, only one set of tolerances, namely, class $\mathrm{X}$, is specified for "not go" gages. The selection of "go" gages from among these classes for use in the inspection of threaded product depends entirely upon the specifications for the product. For example, in the production of parts to class $\mathrm{E}$ specifications, class $\mathrm{X}$ gages may be required for all purposes. On the other hand, for parts made to classes A or B specifications, class Z gages may be sufficiently accurate for all purposes. The recommended uses for the various classes of gages are given in Table 54. In Tables 55, 56, and 57 are specified tolerances on diameter, lead, and angle for each class of gage.

"Go" gages may be permitted to wear to the extreme product limits. It is desirable, however, that working and inspection gages be so selected that the dimensions of the working gages are inside of the limiting dimensions represented by the inspection gages, in order that all parts passed by the working gage will be accepted by the inspection gage. As to wear on "not go" gages, it is purely a question of economy as to when the "not go" gage should be discarded. Continued use reduces the available working tolerance on the product, and the resulting loss must be balanced against the cost of a new gage.

2. Major and Minor Diameters of Gages.-The maximum minor diameter of the "go" ring thread gage should be the same as the minimum minor diameter of the tapped hole. The minimum minor diameter of the "not go" ring thread gage should not be smaller than the maximum minor diameter" of the "go" gage. Also the maximum major diameter of the "not go" plug thread gage should not be larger than the minimum major diameter of the corresponding "go" gage; that is, not larger than the basic major diameter. This will insure contact of the "not go" thread gage on the sides of the threads rather than at the corners of the crest and root.

TABLE 54.-Recommended uses for classes $X, Y$, and $Z$ gages

\begin{tabular}{|c|c|c|c|}
\hline Class of fit & Setting gage & Inspection gage & Working gage \\
\hline 1 & 2 & 3 & 4 \\
\hline 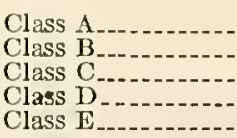 & 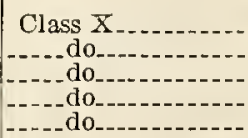 & \begin{tabular}{|l} 
Class $Y$ Y \\
Class $\mathbf{X}$ \\
\end{tabular} & $\begin{array}{c}\text { Class Z. } \\
\text { Do. } \\
\text { Class Y. } \\
\text { Do. } \\
\text { Class X. }\end{array}$ \\
\hline
\end{tabular}


TABLE 55.-Tolerances for class $X$ "go" thread gages and all "not go" thread gages

\begin{tabular}{|c|c|c|c|c|c|c|}
\hline \multirow{2}{*}{ Threads per inch } & \multicolumn{2}{|c|}{$\begin{array}{c}\text { Tolerance on pitch } \\
\text { diameter }\end{array}$} & \multirow{2}{*}{$\begin{array}{l}\text { Tolerance } \\
\text { in lead }\end{array}$} & \multirow{2}{*}{$\begin{array}{c}\text { Tolerance } \\
\text { on half } \\
\text { angle of } \\
\text { thread }\end{array}$} & \multicolumn{2}{|c|}{$\begin{array}{l}\text { Tolerance on major or } \\
\text { minor diameters } 1\end{array}$} \\
\hline & From- & To- & & & From- & To- \\
\hline 1 & 2 & 3 & 4 & 5 & 6 & 7 \\
\hline $\begin{array}{l}64 \\
56 \\
48 \\
46\end{array} 6$ & $\begin{array}{l}\text { Inch } \\
0.0000 \\
.0000 \\
.0000 \\
.0000 \\
.0000\end{array}$ & $\begin{array}{l}\text { Inch } \\
0.0002 \\
.0002 \\
.0002 \\
.0002 \\
.0002\end{array}$ & $\begin{array}{l}\text { Inch } \\
\pm \\
\pm 0.0002 \\
.0002 \\
.0002 \\
.0002 \\
.0002\end{array}$ & $\begin{array}{cc}\text { Deg. } & \text { Min. } \\
\text { 士 } & \\
0 & 30 \\
0 & 30 \\
0 & 30 \\
0 & 20 \\
0 & 20\end{array}$ & $\begin{array}{l}\text { Inch } \\
0.0000 \\
.0000 \\
.0000 \\
.0000 \\
.0000\end{array}$ & $\begin{array}{l}\text { Inch } \\
0.0004 \\
.0004 \\
.0004 \\
.0004 \\
.0004\end{array}$ \\
\hline $\begin{array}{l}32 \ldots \\
28 \ldots \\
24 \ldots \\
20 \ldots \\
18 \ldots\end{array}$ & $\begin{array}{l}.0000 \\
.0000 \\
.0000 \\
.0000 \\
.0000\end{array}$ & $\begin{array}{l}.0003 \\
.0003 \\
.0003 \\
.0003 \\
.0003\end{array}$ & $\begin{array}{l}.0003 \\
.0003 \\
.0003 \\
.0003 \\
.0003\end{array}$ & $\begin{array}{ll}0 & 15 \\
0 & 15 \\
0 & 15 \\
0 & 15 \\
0 & 10\end{array}$ & $\begin{array}{l}.0000 \\
.0000 \\
.0000 \\
.0000 \\
.0000\end{array}$ & $\begin{array}{l}.0004 \\
.0005 \\
.0005 \\
.0005 \\
.0005\end{array}$ \\
\hline 16 & $\begin{array}{l}.0000 \\
.0000 \\
.0000 \\
.0000 \\
.0000\end{array}$ & $\begin{array}{l}.0003 \\
.0003 \\
.0003 \\
.0003 \\
.0004\end{array}$ & $\begin{array}{l}.0003 \\
.0003 \\
.0003 \\
.0003 \\
.0004\end{array}$ & $\begin{array}{ll}0 & 10 \\
0 & 10 \\
0 & 10 \\
0 & 10 \\
0 & 5\end{array}$ & $\begin{array}{l}.0000 \\
.0000 \\
.0000 \\
.0000 \\
.0000\end{array}$ & $\begin{array}{l}.0006 \\
.0006 \\
.0006 \\
.0006 \\
.0007\end{array}$ \\
\hline $6 \ldots$ & $\begin{array}{l}.0000 \\
.0000\end{array}$ & $\begin{array}{l}.0004 \\
.0004\end{array}$ & $\begin{array}{l}.0004 \\
.0004\end{array}$ & $\begin{array}{ll}0 & 5 \\
0 & 5\end{array}$ & $\begin{array}{l}.0000 \\
.0000\end{array}$ & $\begin{array}{l}.0008 \\
.0009\end{array}$ \\
\hline
\end{tabular}

1 On "go" plugs the tolerance is plus, and on "go" rings the tolerance is minus. On "not go" plugs the tolerance is minus, and on "not go" rings the tolerance is plus.

2 Allow able variation in lead between any two threads not farther apart than the length of engagement.

TABLE 56.-Tolerances for class $Y$ "go" thread gages

\begin{tabular}{|c|c|c|c|c|c|c|}
\hline \multirow{2}{*}{ Threads per inch } & \multicolumn{2}{|c|}{$\begin{array}{l}\text { Tolerance on pitch } \\
\text { diameter }\end{array}$} & \multirow{2}{*}{$\begin{array}{l}\text { Tolerance } \\
\text { in lead }{ }^{2}\end{array}$} & \multirow{2}{*}{$\begin{array}{c}\text { Tolerance } \\
\text { on half } \\
\text { angle of } \\
\text { thread }\end{array}$} & \multicolumn{2}{|c|}{$\begin{array}{l}\text { Tolerance on major or } \\
\text { minor diameters } 1\end{array}$} \\
\hline & From- & To- & & & From- & To- \\
\hline 1 & 2 & 3 & 4 & 5 & 6 & 7 \\
\hline $\begin{array}{l}64 \\
56 \\
48 \\
40 \\
36\end{array}$ & $\begin{array}{l}\text { Inch } \\
0.0001 \\
.0001 \\
.0001 \\
.0001 \\
.0001\end{array}$ & $\begin{array}{l}\text { Inch } \\
0.0004 \\
.0004 \\
.0004 \\
.0004 \\
.0004\end{array}$ & $\begin{array}{l}\text { Inch } \\
\pm \\
\pm .0002 \\
0002 \\
.0002 \\
.0002 \\
.0002\end{array}$ & $\begin{array}{cc}\text { Deg. } & \text { Min. } \\
\text { 土 } & \\
0 & 45 \\
0 & 45 \\
0 & 45 \\
0 & 30 \\
0 & 30\end{array}$ & $\begin{array}{l}\text { Inch } \\
0.0000 \\
.0000 \\
.0000 \\
.0000 \\
.0000\end{array}$ & $\begin{array}{l}\text { Inch } \\
\\
0.0004 \\
.0004 \\
.0004 \\
.0004 \\
.0004\end{array}$ \\
\hline $\begin{array}{l}32 \\
28 \\
24 \\
20 \\
18\end{array}$ & $\begin{array}{l}.0001 \\
.0002 \\
.0002 \\
.0002 \\
.0002\end{array}$ & $\begin{array}{l}.0004 \\
.0005 \\
.0005 \\
.0005 \\
.0005\end{array}$ & $\begin{array}{l}.0003 \\
.0003 \\
.0003 \\
.0003 \\
.0003\end{array}$ & $\begin{array}{ll}0 & 20 \\
0 & 20 \\
0 & 20 \\
0 & 20 \\
0 & 15\end{array}$ & $\begin{array}{l}.0000 \\
.0000 \\
.0000 \\
.0000 \\
.0000\end{array}$ & $\begin{array}{l}.0004 \\
.0005 \\
.0005 \\
.0005 \\
.0005\end{array}$ \\
\hline 16 & $\begin{array}{l}.0002 \\
.0002 \\
.0002 \\
.0002 \\
.0002\end{array}$ & $\begin{array}{l}.0006 \\
.0006 \\
.0006 \\
.0006 \\
.0007\end{array}$ & $\begin{array}{l}.0003 \\
.0003 \\
.0003 \\
.0003 \\
.0004\end{array}$ & $\begin{array}{lr}0 & 15 \\
0 & 15 \\
0 & 10 \\
0 & 10 \\
0 & 5\end{array}$ & $\begin{array}{l}.0000 \\
.0000 \\
.0000 \\
.0000 \\
.0000\end{array}$ & $\begin{array}{l}.0006 \\
.0006 \\
.0006 \\
.0006 \\
.0007\end{array}$ \\
\hline 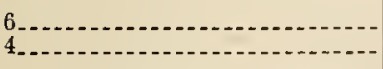 & .0003 & $\begin{array}{l}.0008 \\
.0009\end{array}$ & $\begin{array}{l}.0004 \\
.0004\end{array}$ & $\begin{array}{l}0 \\
0\end{array}$ & $\begin{array}{l}.0000 \\
.0000\end{array}$ & $\begin{array}{l}.0008 \\
.0009\end{array}$ \\
\hline
\end{tabular}

1 On "go" plugs the tolerance is plus, and on "go" rings the tolerance is minus.

Allowable variation in lead between any two threads not farther apart than the leagth of engagement. 
TABLE 57.-Tolerances for class $Z$ "go" thread gages

\begin{tabular}{|c|c|c|c|c|c|c|}
\hline \multirow{2}{*}{ Threads per inch } & \multicolumn{2}{|c|}{$\begin{array}{l}\text { Tolerance on pitch } \\
\text { diameter }\end{array}$} & \multirow{2}{*}{$\begin{array}{l}\text { Tolerance } \\
\text { in lead }{ }^{2}\end{array}$} & \multirow{2}{*}{$\begin{array}{l}\text { Tolerance } \\
\text { on half } \\
\text { angle of } \\
\text { thread }\end{array}$} & \multicolumn{2}{|c|}{$\begin{array}{l}\text { Tolerance on major or } \\
\text { minor diameters }{ }^{1}\end{array}$} \\
\hline & From- & To- & & & From- & To- \\
\hline 1 & - 2 & 3 & 4 & ๖ & 6 & 7 \\
\hline $\begin{array}{l}64 \\
56 \\
48 \\
40 \\
36\end{array}$ & $\begin{array}{l}\text { Inch } \\
0.0002 \\
.0002 \\
.0002 \\
.0002 \\
.0003\end{array}$ & $\begin{array}{l}\text { Inch } \\
0.0006 \\
.0007 \\
.0007 \\
.0007 \\
.0008\end{array}$ & $\begin{array}{l}\text { Inch } \\
\pm \\
0.0002 \\
.0002 \\
.0002 \\
.0002 \\
.0002\end{array}$ & $\begin{array}{cc}\text { Deg. } & \text { Min. } \\
\text { 土 } & \\
0 & 45 \\
0 & 45 \\
0 & 45 \\
0 & 30 \\
0 & 30\end{array}$ & $\begin{array}{l}\text { Inch } \\
0.0000 \\
.0000 \\
.0000 \\
.0000 \\
.0000\end{array}$ & $\begin{array}{l}\text { Inch } \\
0.0004 \\
.0004 \\
.0004 \\
.0004 \\
.0004\end{array}$ \\
\hline $\begin{array}{l}32 \\
28 \\
24 \\
20 \\
18\end{array}$ & $\begin{array}{l}.0003 \\
.0003 \\
.0003 \\
.0003 \\
.0004\end{array}$ & $\begin{array}{l}.0008 \\
.0008 \\
.0009 \\
.0009 \\
.0010\end{array}$ & $\begin{array}{l}.0003 \\
.0003 \\
.0003 \\
.0003 \\
.0004\end{array}$ & $\begin{array}{ll}0 & 20 \\
0 & 20 \\
0 & 20 \\
0 & 20 \\
0 & 15\end{array}$ & $\begin{array}{l}.0000 \\
.0000 \\
.0000 \\
.0000 \\
.0000\end{array}$ & $\begin{array}{l}.0004 \\
.0005 \\
.0005 \\
.0005 \\
.0005\end{array}$ \\
\hline $\begin{array}{l}16 \\
14 \\
12 \\
10\end{array}$ & $\begin{array}{l}.0004 \\
.0004 \\
.0004 \\
.0005 \\
.0006\end{array}$ & $\begin{array}{l}.0010 \\
.0010 \\
.0011 \\
.0012 \\
.0013\end{array}$ & $\begin{array}{l}.0004 \\
.0004 \\
.0004 \\
.0004 \\
.0005\end{array}$ & $\begin{array}{rr}0 & 15 \\
0 & 15 \\
0 & 10 \\
0 & 10 \\
0 & 5\end{array}$ & $\begin{array}{l}.0000 \\
.0000 \\
.0000 \\
.0000 \\
.0000\end{array}$ & $\begin{array}{l}.0006 \\
.0006 \\
.0006 \\
.0006 \\
.0007\end{array}$ \\
\hline 6 & $\begin{array}{l}.0006 \\
.0007\end{array}$ & $\begin{array}{l}.0014 \\
.0016\end{array}$ & $\begin{array}{l}.0005 \\
.0005\end{array}$ & $\begin{array}{l}0 \\
0\end{array}$ & $\begin{array}{l}.0000 \\
.0000\end{array}$ & $\begin{array}{l}.0008 \\
.0009\end{array}$ \\
\hline
\end{tabular}

I On "go" plugs the tolerance is plus, and on "go" rings the tolerance is minus.

Allowable variation in lead between any two threads not farther apart than the length of engagement.

\section{SECTION V. MATIONAL HOSE-COUPLING AND FIRE-HOSE COUPLING THREADS}

Screral years ago specifications for "national standard fire-hose coupling threads" were approved by the National Board of Fire Underwriters, National Fire Protection Association, American Society of Mechanical Engineers, American Socicty of Municipal Improvements, New England Water Works Association, American Water Works Association, the Bureau of Standards, and other interested organizations. These specifications were published in 1911 as the Specifications of the National Board of Fire Underwriters, recommended by the National Fire Protection Association and approved by the various other organizations. They were also published in 1914 as Circular No. 50 of the Bureau of Standards. This circular was revised and republished in 1917.

When the National Serew Thread Commission took up its work on the standardization of screw threads, the specifications for fire-hose coupling threads above referred to were accepted as the basis of its work on fire-hose coupling threads. It was found, howerer, that the specifications as originally drawn were inadequate in that they specified nominal dimensions only, with no maximum and minimum limits.

From the besê information available when fire-hose coupling threads were under discussion, the commission conciuded that they should be placed in the loose-fit class and that the allowance provided in the original specifications should be interpreted as the minimum difference 
in pitch diameter of the couplings and nipples; that is, that a looser fit could be tolerated than that represented by the nominal climensions, but that a tighter fit would be objectionable. The nominal size of the coupling was, therefore, taken as the minimum coupling, and the nominal size of the nipple as the maximum nipple; the loose-fit tolerances being applied in such a direction as to make the maximum coupling larger and the minimum nipple smaller than the nominal size by the amount of the tolerance. The dimensions for "national fire-hose coupling threads" appearing in the 1921 Progress Report of the commission were calculated on that basis.

It later appeared from evidence presented by the National Board of Fire Underwriters that the fits resulting from the limiting dimensions specified in the 1921 Progress Report may in extreme cases be looser than good practice warrants. The commission was, therefore, requested to revise its specifications for fire-hose coupling threads in such a manner that the resulting fits would be somewhat closer than those originally permitted. The revision requested was accomplished by reducing the allowance between the minimum coupling and the maximum nipple; the minimum coupling dimension being retained as published in the 1921 Progress Report. Howerer, from evidence presented by certain manufacturers, it appeared desirable to provide a larger wear limit for gages and also permit the use of field inspection gages which were outside of the limits set for the manufacturers' gages, in order to insure the approval, by the purchaser, of threaded couplings which had passed the manufacturers' gages. To permit this, the tolerances on both the coupling and nipple were increased slightly.

Furthermore, since the publication of the 1921 Progress Report, it has been found desirable, in order to facilitate production and simplify the manufacture of threading tools and gages, to adopt the national form of thread for "national fire-hose coupling threads." This, however, is a very minor change and only slightly affects the thread form at crest and root.

The specifications have been further supplemented by recommended limiting dimensions of gages such that every coupling and nipple accepted will be known to be within the limiting dimensions specified. Only such a system can insure that the finished product will assemble and function properly.

Comparison of the resulting dimensions with those contained in the original specifications of the National Board of Fire Underwriters and in Bureau of Standards Circular No. 50, shows that the two are not in conflict. The nominal dimensions originally specified for couplings and nipples are not inconsistent with the above limiting dimensions, and the revised dimensions should not be considered as changing in any way the original specifications for "national fire-hose coupling threads," but as supplementing those specifications, and as expressing 
them in definite and measurable terms. It should be understood that these dimensions for "national fire-hose coupling threads" are the limiting dimensions for the finished product and not for the threading tools.

With regard to the "national hose-coupling threads," which range in size from three-fourths to 2 inches, the series of sizes and pitches originally adopted by the commission are essentially the same as the corresponding sizes of the "national straight pipe threads," with the exception of the three-fourths-inch size. In this case 11 and $111 / 2$ threads, with a diameter of $1 \frac{1}{16}$ inches, was already in very extensive use. The adoption of the so-called "straight iron pipe thread" was indorsed by the Nation Association of Brass Manufacturers, and at the time of its adoption was recommended by the committee on Small Hose Couplings of the National Fire Protection Association and tentatively adopted by that association.

In ordering threading tools for producing "national hose-coupling and fire-hose coupling threads," it should be pointed out that new taps should be near the maximum permissible size of the coupling, and new dies near the minimum permissible size of the nipple, in order that reasonable wear may be provided. As the threading tools wear by use, the couplings will become smaller and the nipples larger until the limiting dimensions are reached. These must not be exceeded. When the product reaches, or comes dangerously close to the limiting size, the threading tools should be readjusted or replaced.

\section{FORM OF THREAD}

1. Angle of Thread.-The basic angle of thread (A) between the sides of the thread measured in an axial plane is $60^{\circ}$. The line bisecting this $60^{\circ}$ angle is perpendicular to the axis of the screw thread.

2. Flat at CREST and Root. - The flat at the root and crest of the basic thread form is $1 / 8 \times p$, or $0.125 \times p$.

3. Depti of Thread. - The depth of the basic thread form is where

$$
h=0.649519 \times p, \text { or } h=\frac{0.649519}{n}
$$

$$
\begin{aligned}
& p=\text { pitch in inches } \\
& n=\text { number of threads per inch } \\
& h=\text { basic depth of thread. }
\end{aligned}
$$

\section{THREAD SERIES}

(a) National Hose-Coupling Threads.-There is specified in Table 58 a thread series and basic dimensions for hose-coupling threads from three-fourths to 2 inches in diameter, which will be known as the "national hose-coupling threads."

The "national hose-coupling thread" shall be used on all couplings and connections where sizes between three-fourths and 2 inches in diameter are required. 
TABLE 58.-National hose-coupling threads

BASIC MINIMUM COUPLING DIMENSIONS

\begin{tabular}{|c|c|c|c|c|c|c|c|}
\hline Nominal size & $\begin{array}{c}\text { Number of } \\
\text { threads } \\
\text { per inch }\end{array}$ & Pitch & $\begin{array}{c}\text { Depth of } \\
\text { thread }\end{array}$ & $\begin{array}{l}\text { Major } \\
\text { diameter }\end{array}$ & $\begin{array}{c}\text { Pitch } \\
\text { diameter }\end{array}$ & $\begin{array}{l}\text { Minor } \\
\text { diameter }\end{array}$ & Allowance \\
\hline 1 & 2 & 3 & 4 & 5 & 6 & 7 & 8 \\
\hline-1 & $\begin{array}{l}111 / 2 \\
111 / 2 \\
111 / 2 \\
111 / 2 \\
111 / 2\end{array}$ & $\begin{array}{l}\text { Inch } \\
0.08696 \\
.08696 \\
.08696 \\
.08696 \\
.08696\end{array}$ & $\begin{array}{l}\text { Inch } \\
0.05648 \\
.05648 \\
.05648 \\
.05648 \\
.05648\end{array}$ & $\begin{array}{l}\text { Inches } \\
1.0725 \\
1.3051 \\
1.6499 \\
1.8888 \\
2.3628\end{array}$ & $\begin{array}{l}\text { Inches } \\
1.0160 \\
1.2486 \\
1.5934 \\
1.8323 \\
2.3063\end{array}$ & $\begin{array}{l}\text { Inches } \\
0.9595 \\
1.1921 \\
1.5369 \\
1.7758 \\
2.2498\end{array}$ & $\begin{array}{c}\text { Inch } \\
\\
\end{array}$ \\
\hline
\end{tabular}

BASIC MAXIMUM NIPPLE DIMENSIONS

\begin{tabular}{|c|c|c|c|c|c|c|c|}
\hline $\begin{array}{l}2 / 4 \\
1 \\
11 / 4 \\
11 / 2 \\
2\end{array}$ & $\begin{array}{l}111 / 2 \\
111 / 2 \\
111 / 2 \\
111 / 2 \\
111 / 2\end{array}$ & $\begin{array}{r}0.08696 \\
.08696 \\
.08696 \\
.08696 \\
.08696\end{array}$ & $\begin{array}{r}0.05648 \\
.05648 \\
.05648 \\
.05648 \\
.05648\end{array}$ & $\begin{array}{l}\text { 1. } 0625 \\
\text { 1. } 2951 \\
\text { 1. } 6399 \\
\text { 1. } 8788 \\
\text { 2. } 3528\end{array}$ & $\begin{array}{l}1.0060 \\
1.2386 \\
1.5834 \\
1.8223 \\
2.2963\end{array}$ & $\begin{array}{l}0.9495 \\
\text { 1. } 1821 \\
\text { 1. } 5269 \\
\text { 1. } 7658 \\
\text { 2. } 2398\end{array}$ & $\begin{array}{l}0.01 \\
.01 \\
.01 \\
.01 \\
.01\end{array}$ \\
\hline
\end{tabular}

(b) National Fire-Hose Coupling Threads.-There is specified in Table 59 a thread series and basic dimensions for fire-hose couplings from $2 \frac{1}{2}$ to $4 \frac{1}{2}$ inches in diameter which will be known as the "national fire-hose threads." These basic sizes and dimensions correspond in all details to those recommended by the National Fire Protection Association and by the Bureau of Standards.

The "national fire-hose thread" shall be used on all couplings and hydrant connections for fire-protection systems and for all other purposes where hose couplings and connections are required in sizes between $2 \frac{1}{2}$ and $4 \frac{1}{2}$ inches in diameter.

TABLE 59.-National fire-hose coupling threads

BASIC MINIMUM COUPLING DIMENSIONS

\begin{tabular}{|c|c|c|c|c|c|c|c|}
\hline Nominal size & $\begin{array}{c}\text { Number of } \\
\text { threads } \\
\text { per inch }\end{array}$ & Pitch & $\begin{array}{c}\text { Depth of } \\
\text { thread }\end{array}$ & $\begin{array}{c}\text { Major } \\
\text { diameter }\end{array}$ & $\begin{array}{c}\text { Pitch } \\
\text { diameter }\end{array}$ & $\begin{array}{l}\text { Minor } \\
\text { diameter }\end{array}$ & Allowance \\
\hline 1 & 2 & 3 & 4 & 5 & G & 7 & 8 \\
\hline $\begin{array}{l}2.5000_{-} \\
3.0000_{-} \\
3.5000_{-} \\
4.5000_{-}\end{array}$ & $\begin{array}{l}7.5 \\
6.0 \\
6.0 \\
4.0\end{array}$ & $\begin{array}{l}\text { Inch } \\
0.13333 \\
.16667 \\
.16667 \\
.25000\end{array}$ & $\begin{array}{c}\text { Inch } \\
0.08660 \\
.10825 \\
.10825 \\
.16238\end{array}$ & $\begin{array}{l}\text { Inches } \\
3.0836 \\
3.6389 \\
4.2639 \\
5.7859\end{array}$ & $\begin{array}{l}\text { Inches } \\
2.9970 \\
3.5306 \\
4.1556 \\
5.6235\end{array}$ & $\begin{array}{l}\text { Inches } \\
2.9104 \\
3.4223 \\
4.0473 \\
5.4611\end{array}$ & 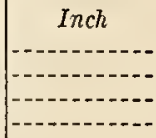 \\
\hline
\end{tabular}

BASIC MAXIMUM NIPPLE DIMENSIONS

\begin{tabular}{|c|c|c|c|c|c|c|c|}
\hline $\begin{array}{l}2.5000 \\
3.0000 \\
3.5000 \\
4.5000\end{array}$ & $\begin{array}{l}7.5 \\
6.0 \\
6.0 \\
4.0\end{array}$ & $\begin{array}{r}0.13333 \\
.16667 \\
.16667 \\
.25000\end{array}$ & $\begin{array}{r}0.08660 \\
.10825 \\
.10825 \\
.16238\end{array}$ & $\begin{array}{l}3.0686 \\
3.6239 \\
4.2439 \\
5.7609\end{array}$ & $\begin{array}{l}2.9820 \\
3.5156 \\
4.1356 \\
5.5985\end{array}$ & $\begin{array}{l}2.8954 \\
3.4073 \\
4.0273 \\
5.4361\end{array}$ & $\begin{array}{r}0.015 \\
.015 \\
.020 \\
.025\end{array}$ \\
\hline
\end{tabular}

\section{ALLOWANCES AND TOLERANCES}

(a) Specified allowances and tolerances, given to Table 60, apply to "national hose-coupling" and "national fire-hose coupling" threads. $16802^{\circ}-25 \dagger-8$ 
The tolerances represent extreme variations permitted on the product. There are shown, in Figure 25, the relations between nipple and coupling dimensions and thread form as specified herein.

(b) The tolerance on the coupling is plus, and is applied from the minimum coupling dimension to above the minimum coupling dimension.

(c) The tolerance on the nipple is minus, and is applied from the maximum nipple dimension to below the maximum nipple dimension.

(d) The pitch diameter tolerances provided for a mating nipple and coupling are the same.

(e) Pitch diameter tolerances include lead and angle variations (see footnote 1, Table 60).
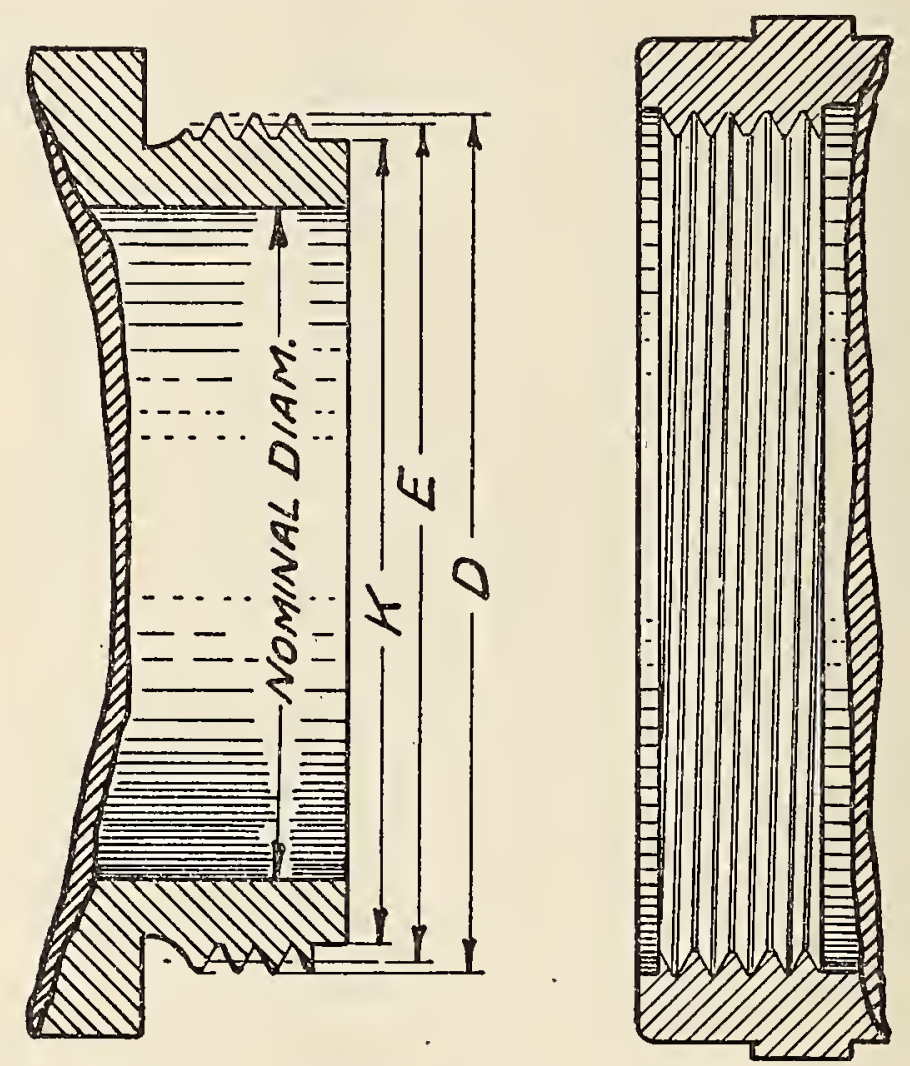

FIG. 24.-National hose-coupling and national fire-hose coupling threads See Tables 60,61 , and 62 for dimensions and toleranees

(f) The tolerance on the major diameter is twice the tolerance on the pitch diameter.

(g) The tolerance on the minor diameter of the nipple is equal to the tolerance on pitch diameter plus two-ninths of the basic thread depth. The minimum minor diameter of a nipple is such as to result in a flat equal to one-third of the basic flat $\left(\frac{1}{24} \times p\right)$ at the root when the pitch diameter of the nipple is at its minimum value. The maximum minor diameter is basic, but may be such as results from the use of a worn or rounded threading tool. 
(h) The tolerance on major diameter of the coupling is equal to the tolerance on pitch diameter plus two-ninths of the basic thread depth. The minimum major diameter of the coupling is such as to result in a basic flat $(1 / 8 \times p)$ when the pitch diameter of the coupling is at its minimum value. The maximum major diameter of the

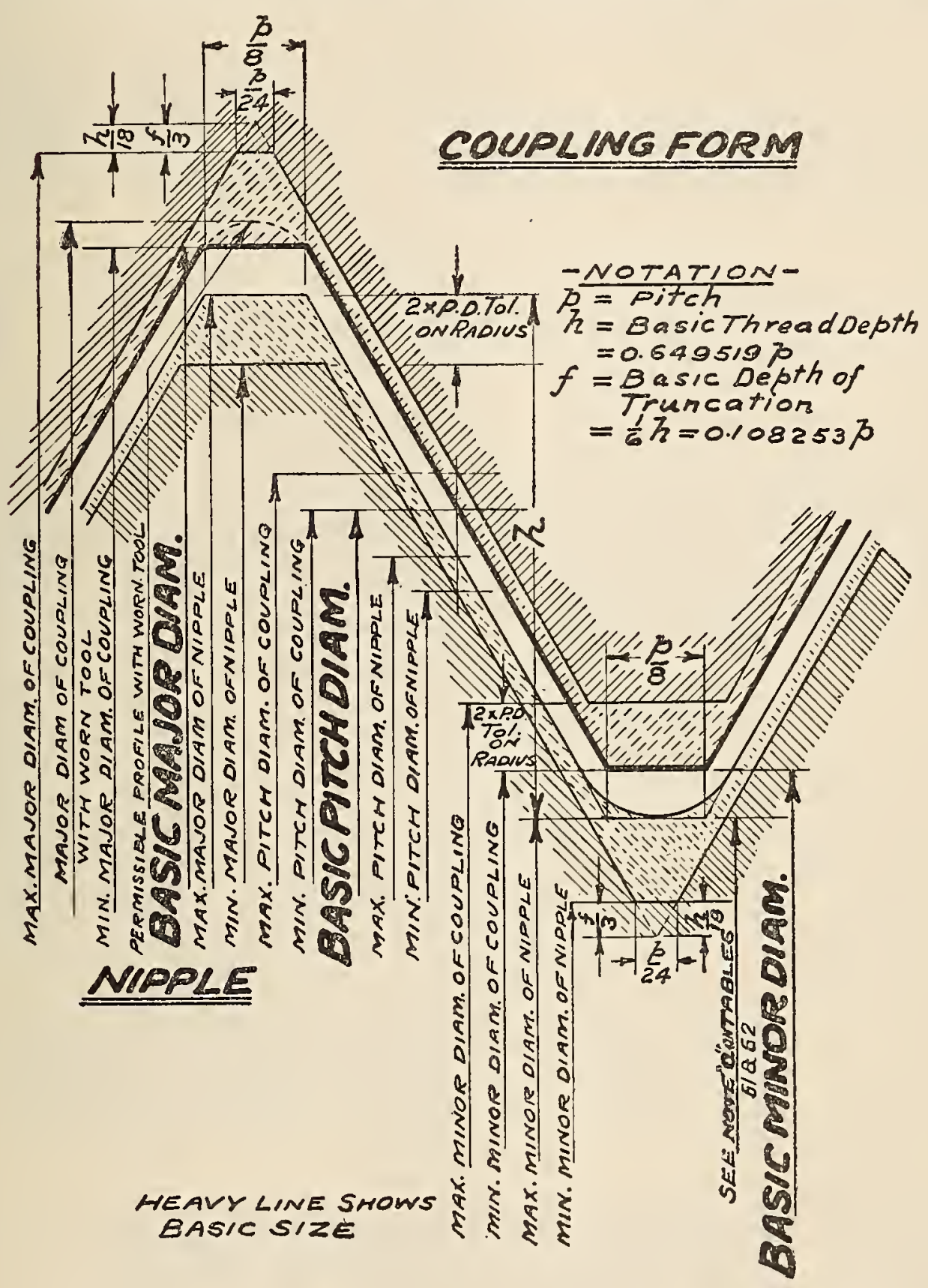

FIG. 25.-National hose-coupling and national fire-hose coupling threads coupling is that corresponding to a flat equal to one-third the basic flat $\left(\frac{1}{24} \times p\right)$.

(i) The tolerance on the minor diameter of the coupling is twice the tolerance on pitch diameter of the coupling. The minimum 
minor diameter of a coupling is such as to result in a basic flat $(1 / 8 \times p)$ at the crest when the pitch diameter of the coupling is at its minimum value.

TABLE 60.-Tolerances and allowances for national hose-coupling and national fire-hose coupling threads

\begin{tabular}{|c|c|c|c|c|c|c|}
\hline Nominal size & $\begin{array}{l}\text { Threads } \\
\text { per inch }\end{array}$ & Allowances & $\begin{array}{l}\text { Tolerances } \\
\text { on pitch } \\
\text { diameter }\end{array}$ & $\begin{array}{c}\text { Lead } \\
\text { errors } \\
\text { consuming } \\
\text { one-balf of } \\
\text { pitch- } \\
\text { diameter } \\
\text { tolerances } 2\end{array}$ & \multicolumn{2}{|c|}{$\begin{array}{c}\text { Errors in } \\
\text { half angle } \\
\text { consuming } \\
\text { one-half of } \\
\text { pitch- } \\
\text { diameter } \\
\text { tolerances }\end{array}$} \\
\hline \multirow[t]{2}{*}{1} & 2 & 3 & 4 & 5 & 6 & \\
\hline & $\begin{array}{l}111 / 2 \\
111 / 2 \\
111 / 2 \\
111 / 2 \\
11 \frac{1}{2}\end{array}$ & $\begin{array}{l}\text { Inch } \\
0.010 \\
.010 \\
.010 \\
.010 \\
.010\end{array}$ & $\begin{array}{l}\text { Inch } \\
0.0085 \\
.0085 \\
.0085 \\
.0085 \\
.0085\end{array}$ & $\begin{array}{l}\text { Inch } \\
0.0025 \\
.0025 \\
.0025 \\
.0025 \\
.0025\end{array}$ & $\begin{array}{c}\text { Deg. } \\
1 \\
1 \\
1 \\
1 \\
1\end{array}$ & $\begin{array}{r}\text { Min. } \\
52 \\
52 \\
52 \\
-52 \\
52\end{array}$ \\
\hline $\begin{array}{l}21 / 2 \ldots \\
31 / 2 \\
41 / 2 \ldots\end{array}$ & $\begin{array}{l}71 / 2 \\
6 \\
6 \\
4\end{array}$ & $\begin{array}{l}.015 \\
.015 \\
.020 \\
.025\end{array}$ & $\begin{array}{l}.0160 \\
.0180 \\
.0180 \\
.0250\end{array}$ & $\begin{array}{l}.0046 \\
.0052 \\
.0052 \\
.0072\end{array}$ & $\begin{array}{l}2 \\
2 \\
2 \\
1\end{array}$ & $\begin{array}{r}17 \\
4 \\
4 \\
55\end{array}$ \\
\hline
\end{tabular}

1 The tolerances specified for pitch diameter include all errors of pitch diameter, lead, and angle. The full tolerance can not therefore he uscd on pitch diameter unless the lead and angle of the thread are perfect. Columns 5 and 6 give, for information, the errors in lead (per length of thread engaged) and in angle, each of which can he compensated for hy half the tolerance on the pitch diameter given in column 4 . If lead and angle errors hotb exist to the amount tabulated, the pitch diameter of a nipple, for example, must be reduced by the full tolerance or it will not enter the "go" gage.

2 Between any two threads not farther apart than the length of engagement.

\section{TABLES OF DIMENSIONS}

TABLE 61.-National hose couplings, thread dimensions

COUPLING THREAD

\begin{tabular}{|c|c|c|c|c|c|c|c|c|c|c|c|c|}
\hline \multirow{2}{*}{$\begin{array}{l}\text { Nominal } \\
\text { size }\end{array}$} & \multirow{2}{*}{$\begin{array}{c}\text { Threads } \\
\text { per } \\
\text { inch }\end{array} \mid$} & \multirow{2}{*}{ Pitch } & \multirow{2}{*}{$\begin{array}{l}\text { Depth } \\
\text { of } \\
\text { thread }\end{array}$} & \multicolumn{3}{|c|}{ Major diameter } & \multicolumn{3}{|c|}{ Pitch diameter } & \multicolumn{3}{|c|}{ Minor diameter } \\
\hline & & & & $\begin{array}{l}\text { Maxi- } \\
\text { mum }\end{array}$ & $\begin{array}{c}\text { Toler- } \\
\text { ance }\end{array}$ & $\begin{array}{l}\text { Mini- } \\
\text { mum }\end{array}$ & $\begin{array}{l}\text { Maxi- } \\
\text { mum }\end{array}$ & $\begin{array}{l}\text { Toler- } \\
\text { ance }\end{array}$ & $\begin{array}{l}\text { Mini- } \\
\text { mum }\end{array}$ & $\begin{array}{l}\text { Maxi- } \\
\text { mum }\end{array}$ & $\begin{array}{l}\text { Toler- } \\
\text { ance }\end{array}$ & $\begin{array}{l}\text { Mini- } \\
\text { mum }\end{array}$ \\
\hline 1 & 2 & 3 & 4 & 5 & 6 & 7 & 8 & 9 & 10 & 11 & 12 & 13 \\
\hline $\begin{array}{l}11 / 2 \\
2 .\end{array}$ & $\begin{array}{l}111 / 2 \\
111 / 2 \\
111 / 2 \\
111 / 2 \\
11 \frac{1}{2}\end{array}$ & $\begin{array}{c}\text { Inch } \\
0.08696 \\
.08696 \\
.08696 \\
.08696 \\
.08696\end{array}$ & $\begin{array}{l}\text { Inch } \\
0.05648 \\
.05648 \\
.05648 \\
.05648 \\
.05648\end{array}$ & Inches & Inch & \begin{tabular}{|l|} 
Inches \\
11.0725 \\
11.3051 \\
11.6499 \\
11.8888 \\
12.3628
\end{tabular} & $\begin{array}{l}\text { Inches } \\
1.0245 \\
1.2571 \\
1.6019 \\
1.8408 \\
2.3148\end{array}$ & $\begin{array}{c}\text { Inch } \\
0.0085 \\
.0085 \\
.0085 \\
.0085 \\
.0085\end{array}$ & $\begin{array}{l}\text { Inches } \\
1.0160 \\
1.2486 \\
1.5934 \\
1.8323 \\
2.3063\end{array}$ & $\begin{array}{c}\text { Inches } \\
0.9765 \\
1.2091 \\
1.5539 \\
1.7928 \\
2.2668\end{array}$ & $\begin{array}{c}\text { Inch } \\
0.0170 \\
.0170 \\
.0170 \\
.0170 \\
.0170\end{array}$ & $\begin{array}{l}\text { Inches } \\
0.9595 \\
1.1921 \\
1.5369 \\
1.7758 \\
2.2498\end{array}$ \\
\hline
\end{tabular}

NIPPLE THREAD

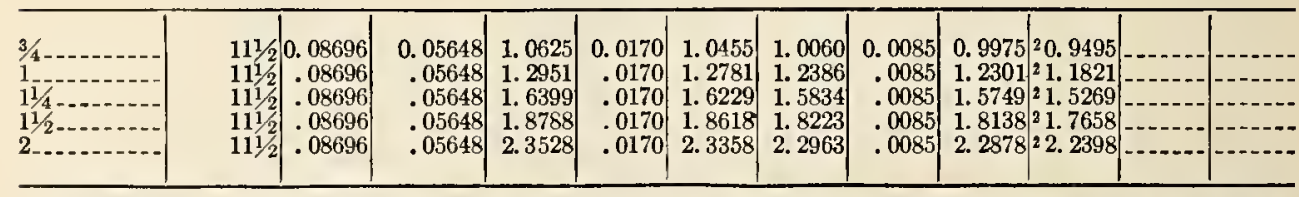

1 Dimensions for the minimum major diameter of the coupling correspond to the basic $f$ lat $(1 / 8 \times p)$, and the profile at the major diameter produced hy a worn tool must not fall below the basic outline. The maximum major diameter of the coupling shall be that corresponding to a flat at the major diameter of

the maximum coupling equal to ${ }_{2}^{1} \times p$.
${ }_{2}$ Dimensions given for the maximum minor diameter of the nipple are figured to the intersection of the worn tool arc with a center line through crest and root. The minimum minor diameter of the nipple shall be that corresponding to a fiat at the minor diameter of the minimum nipple equal to ${ }^{2} \times 2 \times$. 
TABLE 62.-National fire-hose couplings, thread dimensions COUPLING THREAD

\begin{tabular}{|c|c|c|c|c|c|c|c|c|c|c|c|c|}
\hline \multirow{2}{*}{$\begin{array}{c}\text { Nominal } \\
\text { size }\end{array}$} & \multirow{2}{*}{$\begin{array}{c}\text { Threads } \\
\text { per } \\
\text { inch }\end{array}$} & \multirow{2}{*}{ Pitch } & \multirow{2}{*}{$\begin{array}{l}\text { Depth } \\
\text { of } \\
\text { thread }\end{array}$} & \multicolumn{3}{|c|}{ Major diameter } & \multicolumn{3}{|c|}{ Pitch diameter } & \multicolumn{3}{|c|}{ Minor diameter } \\
\hline & & & & $\begin{array}{l}\text { Maxi- } \\
\text { mum }\end{array}$ & $\begin{array}{l}\text { Toler- } \\
\text { ance }\end{array}$ & $\begin{array}{l}\text { Mini- } \\
\text { mum }\end{array}$ & $\begin{array}{l}\text { Maxi- } \\
\text { mum }\end{array}$ & $\begin{array}{c}\text { Toler- } \\
\text { ance }\end{array}$ & $\begin{array}{l}\text { Mini- } \\
\text { mum }\end{array}$ & $\begin{array}{l}\text { Maxi- } \\
\text { mum }\end{array}$ & $\begin{array}{c}\text { Toler- } \\
\text { ance }\end{array}$ & $\begin{array}{l}\text { Mini- } \\
\text { mum }\end{array}$ \\
\hline 1 & 2 & 3 & 4 & 5 & 6 & $\gamma$ & 8 & 9 & 10 & 11 & 12 & 13 \\
\hline $\begin{array}{l}21 / 2-\ldots \\
331 / 2-. \\
31 / 2-- \\
41 / 2-\ldots\end{array}$ & $\begin{array}{l}7.5 \\
6 \\
6 \\
4\end{array}$ & $\begin{array}{c}\text { Inch } \\
0.13333 \\
.16667 \\
.16667 \\
.25000\end{array}$ & $\begin{array}{l}\text { Inch } \\
0.08660 \\
.10825+ \\
.10825+ \\
.16238\end{array}$ & Inches & $\begin{array}{c}\text { Inch } \\
\end{array}$ & $\begin{array}{r}\text { Inches } \\
13.0836 \\
13.6389 \\
14.2639 \\
15.7859\end{array}$ & $\begin{array}{c}\text { Inches } \\
3.0130 \\
3.5486 \\
4.1736 \\
5.6485\end{array}$ & $\begin{array}{c}\text { Inch } \\
0.0160 \\
.0180 \\
.0180 \\
.0250\end{array}$ & \begin{tabular}{|r|} 
Inches \\
2. 9970 \\
3. 5306 \\
4. 1556 \\
5.6235
\end{tabular} & $\begin{array}{r}\text { Inches } \\
2.9424 \\
3.4583 \\
4.0833 \\
5.5111\end{array}$ & $\begin{array}{c}\text { Inch } \\
0.0320 \\
.0360 \\
.0360 \\
.0500\end{array}$ & $\begin{array}{l}\text { Inches } \\
2.9104 \\
3.4223 \\
4.0473 \\
5.4611\end{array}$ \\
\hline
\end{tabular}

NIPPLE THREAD

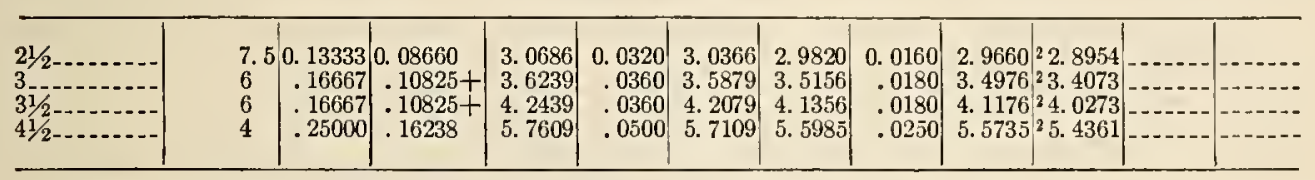

1 Dimensions for the minimum major diameter of the coupling correspond to the basic flat ( $1 / 8 \times p)$, and the profile at the major diameter produced by a worn tool must not fall below the basic outline. The maximum major diameter of the coupling shall be that corresponding to a flat at the major diameter of the maximum coupling equal to $\frac{1}{34} \times p$.

${ }_{2}$ Dimensions given for the maximum minor diameter of the nipple are figured to the intersection of the worn tool arc with a center line through crest and root. The minimum minor diameter of the nipple shall be that corresponding to a flat at the minor diameter of the minimum nipple equal to $\frac{3}{3-5} \times p$

\section{GAGES}

(a) Gages for National Fire-Hose Coupling Threads. - It is recommended that "national fire-hose coupling threads" be inspected in the field by means of gages made within the tolerances given in Table 63. Limiting dimensions for these gages are given in Tables 64 and 65 . These gages will be in the hands of the representative of the purchaser, who will in most cases be the fire chief, or superintendent of water works, or both.

It is further recommended that "national fire-hose coupling threads" be given final inspection by the manufacturer by means of gages made within the limiting dimensions given in Tables 64 and 65, by whatever amount may be desired, in order to avoid, as far as possible, disagreements which might otherwise arise as the result of slight differences in the sizes of gages.

TABLE 63.-Tolerances on gages for national fire-hose coupling threads

\begin{tabular}{|c|c|c|c|c|}
\hline $\begin{array}{c}\text { Allowable variation in lead between any two threads not } \\
\text { farther apart than length of engagement }\end{array}$ & $\begin{array}{c}\text { Allowable } \\
\text { variation } \\
\text { in one-half } \\
\text { angle } \\
\text { thread }\end{array}$ & $\begin{array}{c}\text { Tolerance } \\
\text { on diam- } \\
\text { eter of } \\
\text { minimum } \\
\text { thread } \\
\text { gage }\end{array}$ & $\begin{array}{c}\text { Tolerance } \\
\text { on diam- } \\
\text { eter of } \\
\text { maximum } \\
\text { thread } \\
\text { gage }\end{array}$ \\
\hline 1 & 2 & 3 & 4 \\
\hline Inch & Deg. Min. \\
$\pm 0 \quad 10$ & $\begin{array}{r}\text { Inch } \\
+0.000 \\
+.001\end{array}$ & $\begin{array}{r}\text { Inch } \\
-.000 \\
-.001\end{array}$ \\
\hline
\end{tabular}


TABLE 64.-Limiting dimensions of field inspection plug thread gages for couplings $(\text { internal })^{1}$

\begin{tabular}{|c|c|c|c|c|c|c|c|c|c|}
\hline \multirow{3}{*}{$\begin{array}{c}\text { Nominal } \\
\text { size }\end{array}$} & \multirow{3}{*}{$\begin{array}{l}\text { Threads } \\
\text { per inch }\end{array}$} & \multicolumn{4}{|c|}{ "Go" or minimum gage } & \multicolumn{4}{|c|}{ "Not go" or maximum gage } \\
\hline & & \multicolumn{2}{|c|}{ Major diameter } & \multicolumn{2}{|c|}{ Pitch diameter } & \multicolumn{2}{|c|}{ Major diameter } & \multicolumn{2}{|c|}{ Pitch diameter } \\
\hline & & $\begin{array}{l}\text { Maxi- } \\
\text { mum }\end{array}$ & $\begin{array}{l}\text { Mini- } \\
\text { mum }\end{array}$ & $\begin{array}{l}\text { Maxi- } \\
\text { mum }\end{array}$ & $\begin{array}{l}\text { Mini- } \\
\text { mum }\end{array}$ & $\begin{array}{l}\text { Maxi- } \\
\text { mum }\end{array}$ & $\begin{array}{l}\text { Mini- } \\
\text { mum }\end{array}$ & $\begin{array}{l}\text { Maxi- } \\
\text { mum }\end{array}$ & $\begin{array}{l}\text { Mini- } \\
\text { mum }\end{array}$ \\
\hline 1 & 2 & 3 & 4 & 5 & 6 & y & 8 & 9 & 10 \\
\hline $\begin{array}{l}2.500 \\
3.000 \\
3.500 \\
4.500\end{array}$ & $\begin{array}{l}7.5 \\
6.0 \\
6.0 \\
4.0\end{array}$ & $\begin{array}{c}\text { Inches } \\
3.0816 \\
3.6399 \\
4.2649 \\
5.7869\end{array}$ & $\begin{array}{c}\text { Inches } \\
3.0836 \\
3.6389 \\
4.2639 \\
5.7859\end{array}$ & $\begin{array}{l}\text { Inches } \\
2.9980 \\
3.5316 \\
4.1566 \\
5.6245\end{array}$ & $\begin{array}{c}\text { Inches } \\
2.9970 \\
3.5306 \\
4.1556 \\
5.6235\end{array}$ & $\begin{array}{c}\text { Inches } \\
3.0836 \\
3.6389 \\
4.2639 \\
5.7859\end{array}$ & $\begin{array}{c}\text { Inches } \\
3.0826 \\
3.6379 \\
4.2629 \\
5.7849\end{array}$ & $\begin{array}{c}\text { Inches } \\
3.0130 \\
3.5486 \\
4.1736 \\
5.6485\end{array}$ & $\begin{array}{l}\text { Inches } \\
3.0120 \\
3.5476 \\
4.1726 \\
5.6475\end{array}$ \\
\hline
\end{tabular}

1 The minor diameters of plug gages and the major diameters of ring gages are undercut berond the nominal diameters to give clearance for grinding or lapping. The allowable variation in lead between any two threads not farther apart than the length of engagement is \pm 0.0005 inch. The allowable variation in one-half angle of thread is \pm 10 minutes.

TABLE 65.-Limiting dimensions of field inspection ring thread gages for coupling nipples (external) 1

\begin{tabular}{|c|c|c|c|c|c|c|c|c|c|}
\hline \multirow{3}{*}{$\underset{\text { size }}{\text { Nominal }}$} & \multirow{3}{*}{$\begin{array}{l}\text { Threads } \\
\text { per inch }\end{array}$} & \multicolumn{4}{|c|}{ "Go" or maximum gage } & \multicolumn{4}{|c|}{ "Not go" or minimum gage } \\
\hline & & \multicolumn{2}{|c|}{ Pitch diameter } & \multicolumn{2}{|c|}{ Minor diameter } & \multicolumn{2}{|c|}{ Pitch diameter } & \multicolumn{2}{|c|}{ Minor diameter } \\
\hline & & $\begin{array}{l}\text { Maxi- } \\
\text { mum }\end{array}$ & $\begin{array}{l}\text { Mini- } \\
\text { mum }\end{array}$ & $\begin{array}{l}\text { Maxi- } \\
\text { mum }\end{array}$ & $\begin{array}{l}\text { Mini- } \\
\text { mum }\end{array}$ & $\begin{array}{l}\text { Maxi- } \\
\text { mum }\end{array}$ & $\begin{array}{l}\text { Mini- } \\
\text { mum }\end{array}$ & $\begin{array}{l}\text { Maxi- } \\
\text { mumim }\end{array}$ & $\begin{array}{l}\text { Mini- } \\
\text { mum }\end{array}$ \\
\hline 1 & 2 & 3 & 4 & 5 & 6 & 7 & 8 & 9 & 10 \\
\hline $\begin{array}{l}2.500=- \\
3.000= \\
3.500=- \\
4.500=-\end{array}$ & $\begin{array}{l}7.5 \\
6.0 \\
6.0 \\
4.0\end{array}$ & $\begin{array}{c}\text { Inches } \\
2.9820 \\
3.5156 \\
4.1356 \\
5.5985\end{array}$ & $\begin{array}{c}\text { Inches } \\
2.9810 \\
3.5146 \\
4.1346 \\
5.5975\end{array}$ & $\begin{array}{c}\text { Inches } \\
2.9104 \\
3.4223 \\
4.0473 \\
5.4611\end{array}$ & $\begin{array}{l}\text { Inches } \\
2.9094 \\
3.4213 \\
4.0463 \\
5.4601\end{array}$ & $\begin{array}{l}\text { Inches } \\
2.9670 \\
3.4986 \\
4.1186 \\
5.5745\end{array}$ & $\begin{array}{c}\text { Inches } \\
2.9660 \\
3.4976 \\
4.1176 \\
5.5735\end{array}$ & $\begin{array}{c}\text { Inches } \\
2.9114 \\
3.4233 \\
4.0483 \\
5.4621\end{array}$ & $\begin{array}{l}\text { Inches } \\
2.9104 \\
3.4223 \\
4.0473 \\
5.4611\end{array}$ \\
\hline
\end{tabular}

1 The minor diameters of plug gages and the major diameters of ring gages are undercut beyond the nominal diameters to give clearance for grinding or lapping. The allowable variation in lead between any two threads not farther apart than the length of engagement is \pm 0.0005 inch. The allowable variation in one-half angle of thread is \pm 10 minutes.

\section{SECTION VI. NATIONAL PIPE THREADS}

The material on the subject of pipe threads presented herewith is essentially the same as that in the report prepared by a special committee of the Committee of Manufacturers on Standardization of Fittings and Valves, acting in cooperation with pipe and gage manufacturers and the A. S. M. E. committee on International Standards for Pipe Threads. It was published in October, 1919, under the title "Manual on American Standard Pipe Threads." It has been indorsed by the American Society of Mechanical Engineers and the American Gas Association, and is adopted by the commission with only such changes as are necessary to bring it into conformity with the remainder of the report. 
The "national (American Briggs') pipe-thread standard" for taper threaded pipe joints was formulated prior to the year 1882 by Robert Briggs, of Philadelphia, Pa. This standard, with certain modifications and additions, is now in general use throughout the United States and Canada.

\section{FORM OF THREAD}

(a) Specifications.-1. Angle of thread.-The angle between the sides of the thread is $60^{\circ}$ when measured in an axial plane, and the line bisecting this angle is perpendicular to the axis of the pipe for either taper or straight threads.

2. Depth of thread. ${ }^{10}$ - The crest and root of the thread form are truncated an amount equal to $0.033 p$; the depth of thread is, therefore, equal to $0.8 \mathrm{p}$.

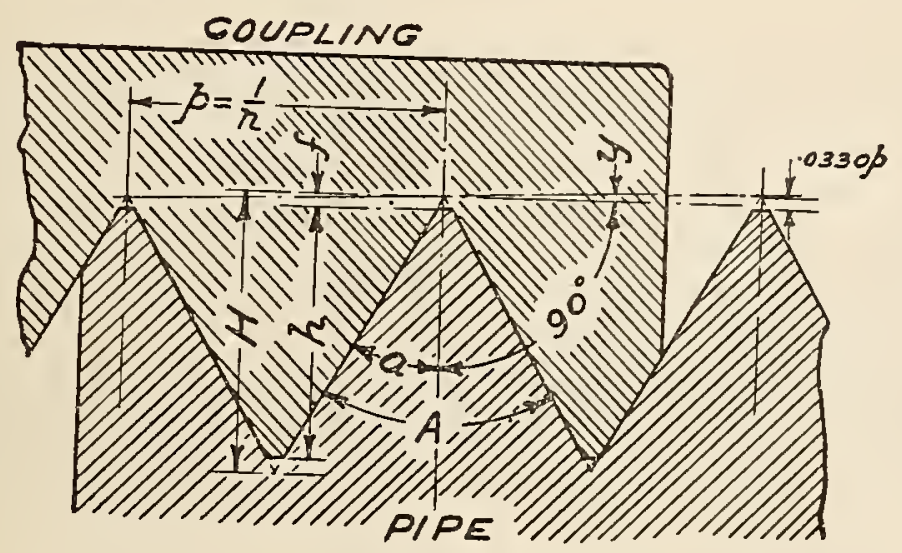

FIG. 26.-National taper pipe thread form and notation

NOTATION

$$
\begin{aligned}
& A=60^{\circ} \text { angle of thread } \\
& a=30^{\circ} \text { one-half angle of thread } \\
& y=1^{\circ} 47^{\prime} \text { approx. taper angle }=\text { one-sixteenth inch per inch on diameter } \\
& H=0.866025 p \text { depth of } 60^{\circ} \text { sharp } \vee \text { thread } \\
& h\left\{\begin{array}{l}
=0.800000 \mathrm{p} \\
=0.923761 \mathrm{H}
\end{array}\right\} \text { depth of thread on work } \\
& f\left\{\begin{array}{l}
=0.033012 \\
=0.038120 \\
H
\end{array}\right\} \text { Depth of truncation } \\
& =0.041266 h\} \\
& \begin{array}{l}
p=1 / n \text { pitch } \\
n=\text { number of threads per inch }
\end{array}
\end{aligned}
$$

3. Taper of thread.-The taper of the thread is 1 in 16 , or threefourths inch per foot, measured on the diameter.

(b) Illustration.-There are shown in Figure 26 the relations as specified herein for form of thread, and general notation. Special notation is given in Figures 27, 28, and 30.

${ }_{10}$ While Mr. Briggs originally advocated a slightly rounded crest and root, cutting tools are actually slightly flattened at the crest and root. 


\section{SYMBOLS}

The list of symbols given in Section II-2, together with additional symbols given below, should be used in formulas for expressing relations of pipe threads and for use on drawings, etc.

Pitch diameter of thread at end of pipe

Pitch diameter of thread at gaging notch

Pitch diameter of thread at $L_{2}$ from end of pipe

Maximum pitch diameter, external locknut thread.

Minimum pitch diameter, internal locknut thread

Distance from gaging notch to end of pipe $=$ normal engagement by hand

Length of effective thread

Nominal outside diameter of pipe=major diameter of pipe thread at $L_{2}$ from

end of pipe.

Internal diameter of pipe

\section{THREAD SERIES}

(a) National (American Briggs') Taper Pipe Threads.-Taper external and internal pipe threads are recommended for threaded pipe joints and pipe fittings for any service. The sizes and basic dimensions of the "national (American Briggs') taper pipe threads" are specified in Table 66.

1. Outside diameter of pipe.-The outside diameters of pipe are given in column 5 of Table 66.

2. Diameters of taper threads.-The pitch diameter's of the taper threads are determined by formulas based on the outside diameter of pipe and the pitch of thread. These are as follows ${ }^{11}$ (see Symbols above):

$$
\begin{aligned}
& E_{0}=D-(0.05 D+1.1) p \\
& E_{1}=E_{0}+0.0625 L_{1}
\end{aligned}
$$

3. Length of thread.-The length of the taper external thread is determined by a formula based on the outside diameter of pipe and the pitch of the thread. This is as follows ${ }^{11}$ (see Symbols above):

$$
L_{2}=(0.8 D+6.8) p
$$

4. Length of engagement.-The normal length of engagement between taper external and internal threads, when screwed together by hand, is shown in column 6 of Table 66 . This length is controlled by means of gages.

5. Tolerances.-The tolerance on diameter is the equivalent of the variation in diameter due to taper over one and one-half turns either way from the basic dimensions. ${ }^{12}$

11 These formulas are not expresscd in the same terms as the formulas originally established by Mr. Briggs, because they aro used to determine directly the pitch diameter and the length of effective thread, which includes two threads slightly imperfect at the crest; whereas tho Briggs formulas determined the major diameter and tho length of perfect thread, the two threads imperfect on the crest not being included in the formula. However, hoth forms give identical results.

${ }_{12}$ On account of tho gage tolerance of one-half turn on working gages, this is equivalent to one turn plus or one turn minus from the gaging notch when using working gagas (see figs. 34 and 35). 


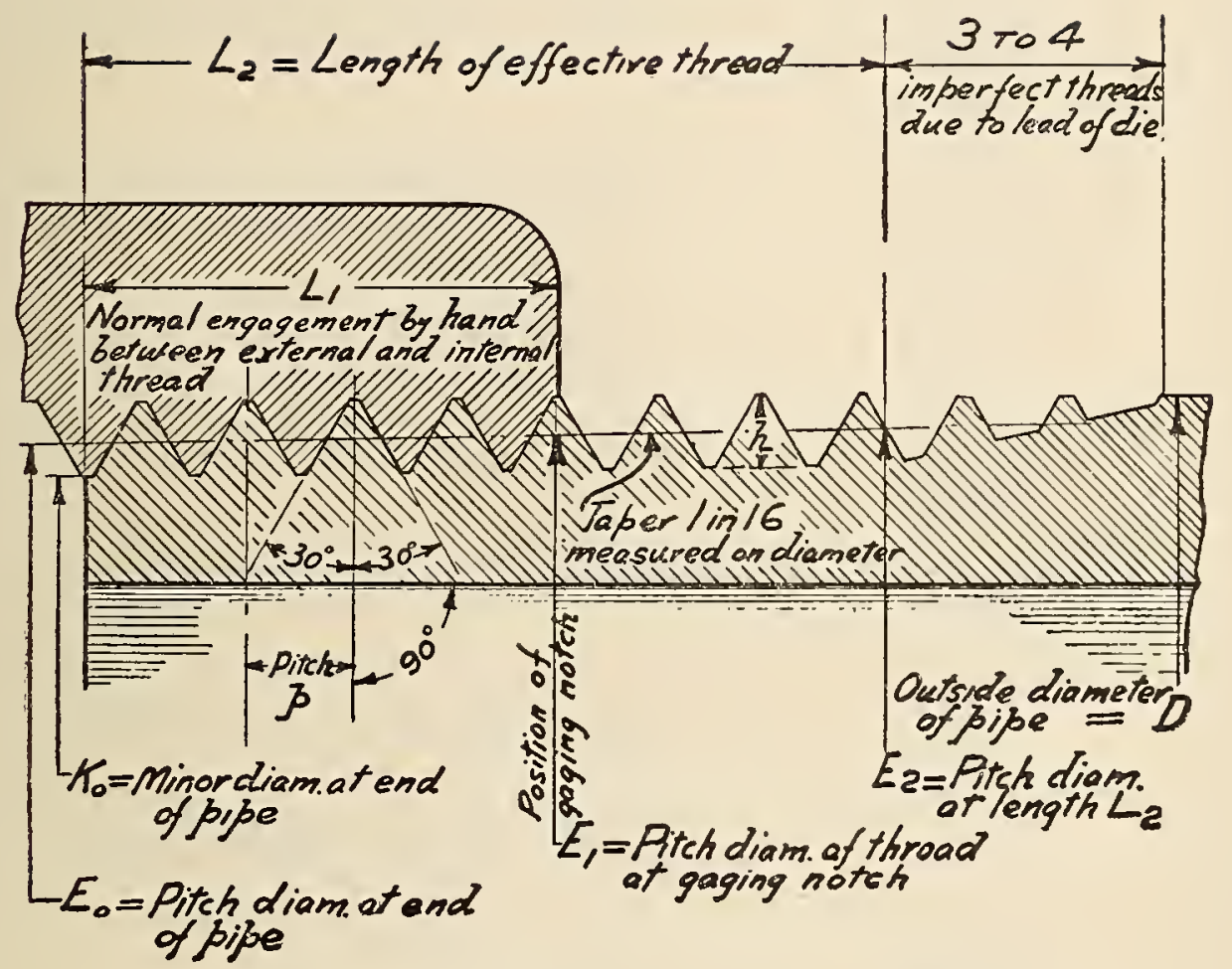

FIg. 27.-National taper pipe thread notation NOTATION

$E_{0}=D-(0.05 D+1.1) p$

$E_{1}=E_{\mathrm{o}}+0.0625 \mathrm{Li}$

$\begin{aligned} L_{h} & =p(0.8 D+6.8) \\ h & =0.8 p\end{aligned}$ 


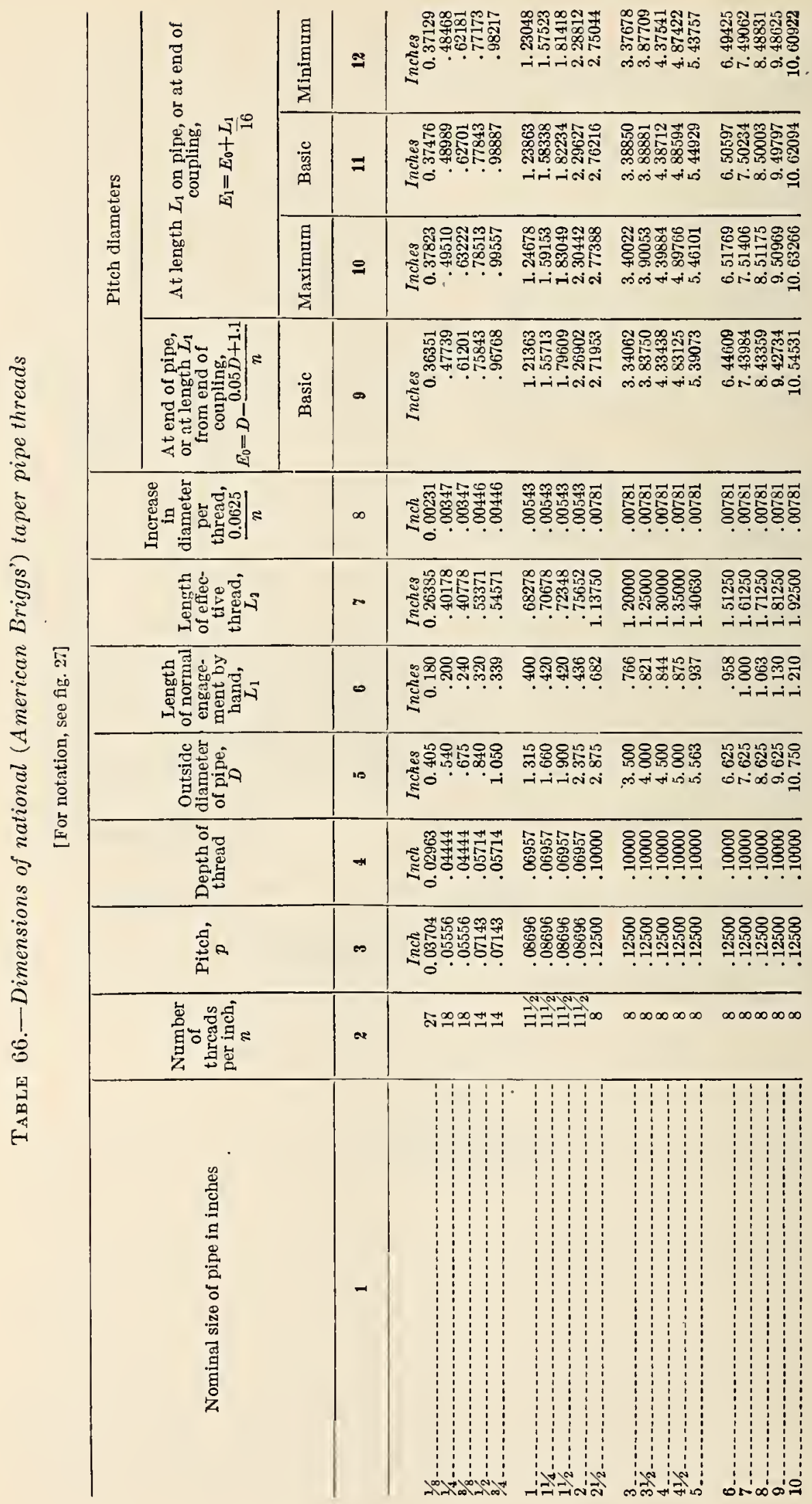




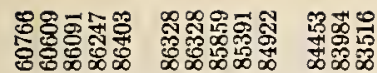

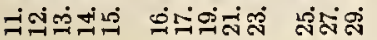

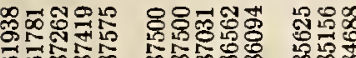

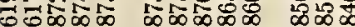

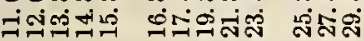

Opg

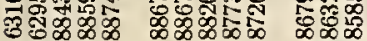

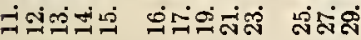

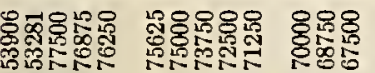

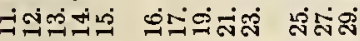

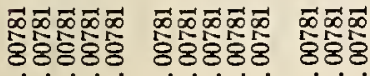

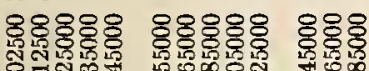

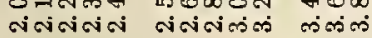

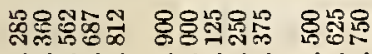

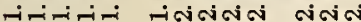

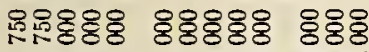

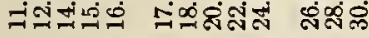

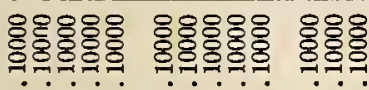

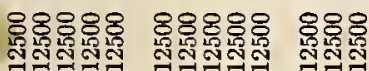

$\infty \infty \infty \infty \infty \quad \infty \infty \infty \infty \infty \infty \infty \infty \infty$

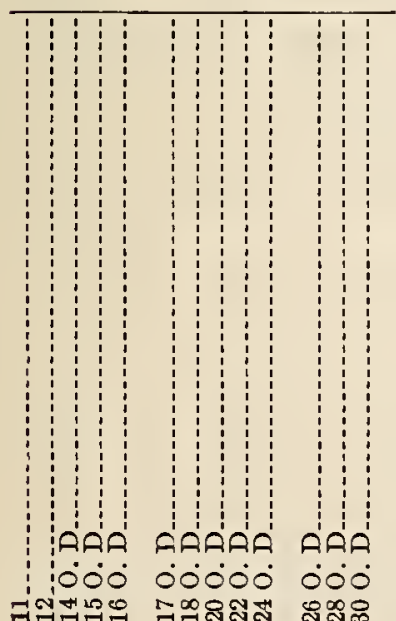


(b) National Straight Pipe Threads.-The specified sizes and basie dimensions on the "national straight pipe threads" are given in Table 67.

1. Diameters of straight threads.-The basie piteh diameter of the straight thread is equal to the diameter at the gaging noteh of "national (Ameriean Briggs') taper pipe threads," and is determined by the following formula based on the outside diameter of pipe and the piteh of thread (see Symbols above):

$$
E_{1}=D-(0.05 D+1.1) p+0.0625 L_{1}
$$

2. Tolerances.-The toleranee on piteh diameter of a straight pipe thread is the equivalent of the variation in diameter over one and one-half turns either way from the gaging noteh of the "national (American Briggs') taper pipe thread." ${ }^{13}$ (See columns 4 and 6 of Table 67.)

3. Application to internal threads.-Straight threaded internal wrought iron or wrought steel eouplings of the weight known as

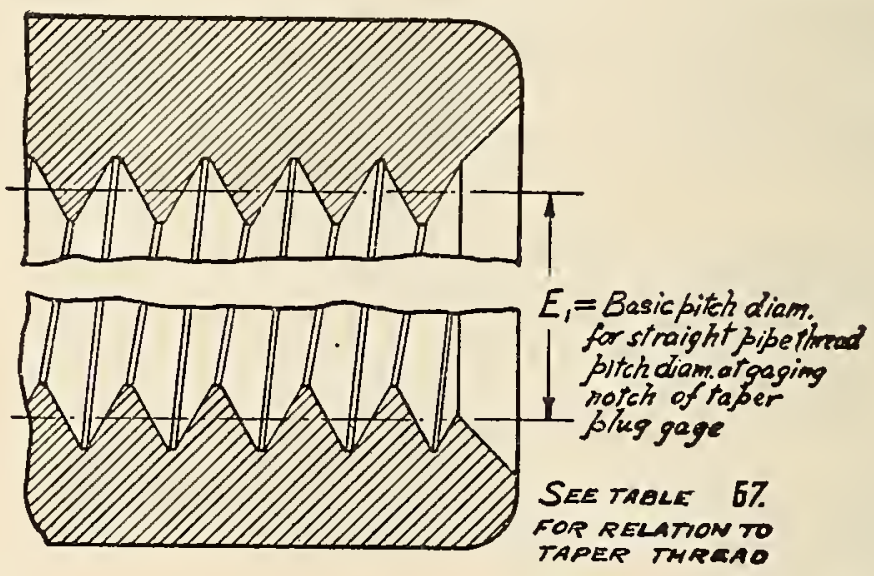

FIG. 28.--National straight pipe thread notation (internal)

Note.-This thread is gaged with the taper threaded plug gage and should gage flush at the face with the gaging notch, allowing a maximum variation of one turn plus or minus from the notch

"standard" may be used with taper threaded pipe for ordinary pressures, as they are sufficiently duetile to adjust themselves to the taper external thread when properly serewed together.

For high pressures, only taper external and internal threads should be used.

4. Application to external threads.-Straight external threads are reeognized only for special applieations, sueh as long serews and tank nipples.

5. Application to long screw joints.-Long serew joints are used to a limited extent. This joint is not considered satisfactory when

18 The coupling thread may be gaged with a taper threaded plug gage. On acrount of the gage tolerance of one-half turn on working taper pipe thread gages, the working tolerance is equivalent to one turn either way from the gaging notch. In gaging, care must be taken to gage at the first thread and not at the end of the coupling when the thread is chamfered. 
subjected to high temperature or pressure. In this application the coupling has a straight thread and must make a joint with a "national taper pipe thread." (See fig. 28.) It is necessary that the coupling be screwed on the straight external thread for the full length of the coupling and then back until it engages the taper external thread. The straight thread on the pipe enters the coupling freely by hand, the joint being made by a packing material between the locknut and the coupling. (See fig. 29.)

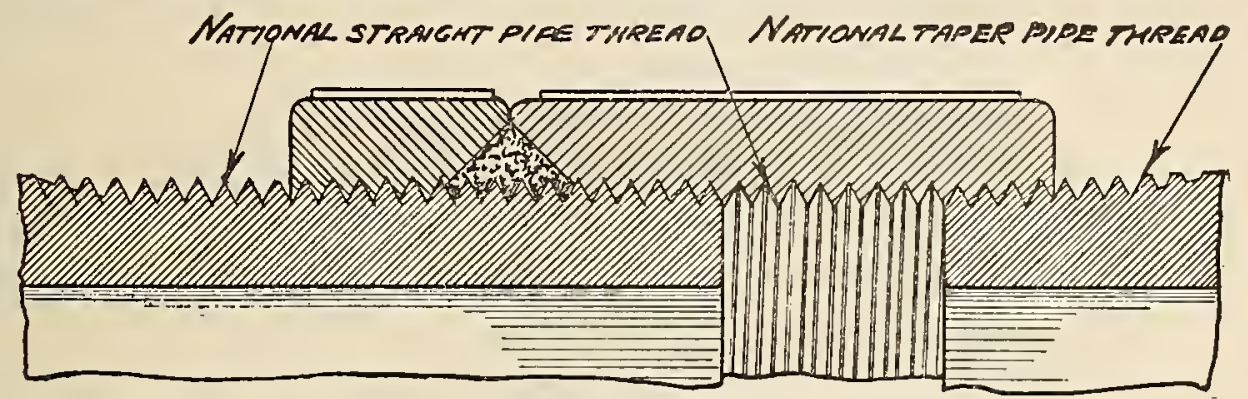

FIG. 29.-Illustration of "long screw" joint between straight threaded coupling and taper threaded pipe

On account of the long engagement of thread, imperfections in pitch affect the fit when the coupling is screwed on the pipe its full length. Refinements of manufacture and gaging to insure a properly interchangeable product are more costly than the commercial use warrants; therefore, the use of this type of joint is not recommended. For this reason, specifications for tolerances and gaging are not included herein.

TABLE 67.-Dimensions of national straight pipe threads (for couplings)

[For notation, see fig. 28]

\begin{tabular}{|c|c|c|c|c|c|c|}
\hline \multirow{2}{*}{ Nominal sizes in inches } & \multirow{2}{*}{$\begin{array}{l}\text { Threads } \\
\text { per inch }\end{array}$} & \multirow{2}{*}{$\begin{array}{l}\text { Major } \\
\text { diameter,1 } \\
\text { basic }\end{array}$} & \multicolumn{3}{|c|}{ Pitch diameter } & \multirow{2}{*}{$\frac{\begin{array}{c}\text { Minor } \\
\text { diameter } 1\end{array}}{\text { Basic }}$} \\
\hline & & & Maximum & Basic & Minimum & \\
\hline \multirow[t]{2}{*}{1} & 2 & 3 & 4 & 5 & 6 & 7 \\
\hline & $\begin{array}{l}27 \\
18 \\
18 \\
14 \\
\cdot 14\end{array}$ & $\begin{array}{l}\text { Inches } \\
0.40439 \\
.53433 \\
.67145 \\
.83557 \\
1.04600\end{array}$ & $\begin{array}{c}\text { Inches } \\
0.37823 \\
.49510 \\
.63222 \\
.78513 \\
.99557\end{array}$ & $\begin{array}{l}\text { Inches } \\
0.37476 \\
.48989 \\
.62701 \\
.7743 \\
.98887\end{array}$ & $\begin{array}{c}\text { Inches } \\
0.37129 \\
.48468 \\
.62181 \\
.77173 \\
.98217\end{array}$ & $\begin{array}{l}\text { Inches } \\
0.34513 \\
.44544 \\
.58257 \\
.72129 \\
.93172\end{array}$ \\
\hline $111 / 42 \ldots$ & $\begin{array}{l}111 / 2 \\
111 / 2 \\
111 / 2 \\
111 / 2 \\
8\end{array}$ & $\begin{array}{l}1.30819 \\
1.65294 \\
1.89190 \\
2.36583 \\
2.86216\end{array}$ & $\begin{array}{l}1.24678 \\
1.59153 \\
1.83049 \\
2.30442 \\
2.77388\end{array}$ & $\begin{array}{l}1.23863 \\
1.58338 \\
1.82234 \\
2.29627 \\
2.76216\end{array}$ & $\begin{array}{l}1.23048 \\
1.57523 \\
1.81418 \\
2.28812 \\
2.75044\end{array}$ & $\begin{array}{l}1.16907 \\
1.51382 \\
1.75277 \\
2.22671 \\
2.66216\end{array}$ \\
\hline $\begin{array}{l}3 \\
31 / 2 \\
41 / 2 \\
5\end{array}$ & $\begin{array}{l}8 \\
8 \\
8 \\
8 \\
8\end{array}$ & $\begin{array}{l}\text { 3. } 48850 \\
\text { 3. } 98881 \\
4.48713 \\
4.98544 \\
5.54929\end{array}$ & $\begin{array}{l}3.40022 \\
3.90053 \\
4.39884 \\
4.89766 \\
5.46101\end{array}$ & $\begin{array}{l}3.38850 \\
3.88881 \\
4.38712 \\
4.88594 \\
5.44929\end{array}$ & $\begin{array}{l}3.37678 \\
3.87709 \\
4.37541 \\
4.87422 \\
5.43757\end{array}$ & $\begin{array}{l}\text { 3. } 28850 \\
3.78881 \\
4.28713 \\
4.78594 \\
5.34929\end{array}$ \\
\hline $6 \ldots$ & 8 & 6.60597 & 6.51769 & 6. 50597 & 6.49425 & 6. 40597 \\
\hline
\end{tabular}

1 The national pipe thread form is maintained; therefore, the major and minor diameters vary with the pitch diameter and are determined by the threading tools. 
(c) National Locknut Threads.-Occasional requirements make it advisable to have a straight thread of the largest diameter it is possible to cut on a pipe. This practice has been standardized and is known as "maximum external and minimum internal locknut threads." For dimensions, see Table 68. The "tank nipple" shown in Figure 31 is an example of this thread. In this application a "national (American Briggs') standard taper pipe thread" is cut on the end of the pipe after having first cut the "external locknut thread."

TABLE 68.-Dimensions of national locknut threads

[For notation, see fig. 30]

\begin{tabular}{|c|c|c|c|c|}
\hline Nominal size in inches & $\begin{array}{l}\text { Threads } \\
\text { per inch }\end{array}$ & $\begin{array}{c}E_{\mathrm{e}} \\
\text { (Maxi- } \\
\text { mum) } 1\end{array}$ & $\begin{array}{c}E_{\mathrm{i}} \\
\text { (Mini- } \\
\text { mum) } 1\end{array}$ & $\begin{array}{l}\text { Depth of } \\
\text { thread }\end{array}$ \\
\hline 1 & 2 & 3 & 4 & 5 \\
\hline $\begin{array}{ll}1 / 8 \\
1 / 4 \\
3 / 8 \\
1 / 8 \\
3 / 4 \\
3 / 2-\cdots\end{array}$ & $\begin{array}{l}27 \\
18 \\
18 \\
14 \\
14\end{array}$ & $\begin{array}{l}\text { Inches } \\
0.38402 \\
.50378 \\
.64090 \\
.79629 \\
1.00672\end{array}$ & $\begin{array}{l}\text { Inches } \\
0.38633 \\
.50725 \\
.64437 \\
.80075 \\
1.01119\end{array}$ & $\begin{array}{l}\text { Inch } \\
0.02963 \\
.04444 \\
.04444 \\
.05714 \\
.05714\end{array}$ \\
\hline $\begin{array}{l}1 \\
11 \\
11\end{array}$ & $\begin{array}{l}111 / 2 \\
111 / 2 \\
111 / 2 \\
111 / 2 \\
8\end{array}$ & $\begin{array}{l}1.26037 \\
1.60512 \\
1.84408 \\
2.31801 \\
2.79341\end{array}$ & $\begin{array}{l}1.26580 \\
1.61055 \\
1.84951 \\
2.32345 \\
2.80122\end{array}$ & $\begin{array}{l}.06957 \\
.06957 \\
.06957 \\
.06957 \\
.10000\end{array}$ \\
\hline $\begin{array}{l}31 \\
3122 \\
41 \\
4\end{array}$ & $\begin{array}{l}8 \\
8 \\
8 \\
8 \\
8\end{array}$ & $\begin{array}{l}\text { 3. } 41975 \\
\text { 3. } 92006 \\
4.41838 \\
\text { 4. } 91719 \\
5.48054\end{array}$ & $\begin{array}{l}3.42756 \\
3.92787 \\
4.42619 \\
4.92500 \\
5.48836\end{array}$ & $\begin{array}{l}.10000 \\
.10000 \\
.10000 \\
.10000 \\
.10000\end{array}$ \\
\hline $\begin{array}{l}6 \\
8 \\
8 \\
8\end{array}$ & $\begin{array}{l}8 \\
8 \\
8 \\
8 \\
8\end{array}$ & $\begin{array}{r}6.53722 \\
7.53359 \\
8.53128 \\
9.52922 \\
10.65219\end{array}$ & $\begin{array}{r}6.54503 \\
7.54141 \\
8.53909 \\
9.53703 \\
10.66000\end{array}$ & $\begin{array}{l}.10000 \\
.10000 \\
.10000 \\
.10000 \\
.10000\end{array}$ \\
\hline 112 & $\begin{array}{l}8 \\
8\end{array}$ & $\begin{array}{l}11.65063 \\
12.64906\end{array}$ & $\begin{array}{l}11.65844 \\
12.65688\end{array}$ & $\begin{array}{l}.10000 \\
.10000\end{array}$ \\
\hline
\end{tabular}

${ }^{1}$ A tolerance equivalent to one and one-half turns of the "national taper pipe thread" is recommended, the tolerance being minus on $E_{\mathrm{e}}$ and plus on $E_{\mathrm{i}}$. 


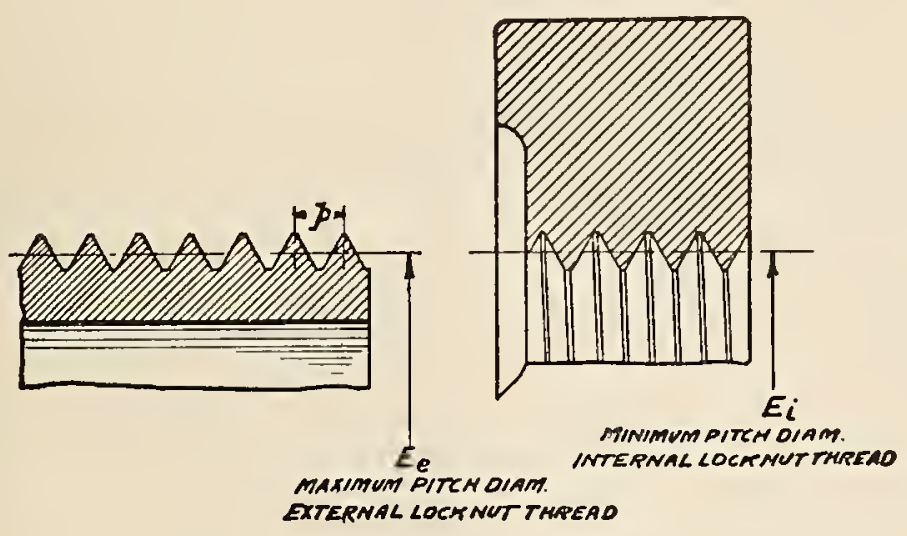

FIa. 30.-National lo:knut thread notation NOTATION

$E_{1}=$ pitch diameter at gaging notch of national taper plug gage $E_{\mathrm{e}}=E_{1}+(4 p \times 0.0625)$
$E_{\mathrm{i}}=E_{1}+(5 p \times 0.0625)$

NoTE.-See Table 68 for relation to taper pipe thread

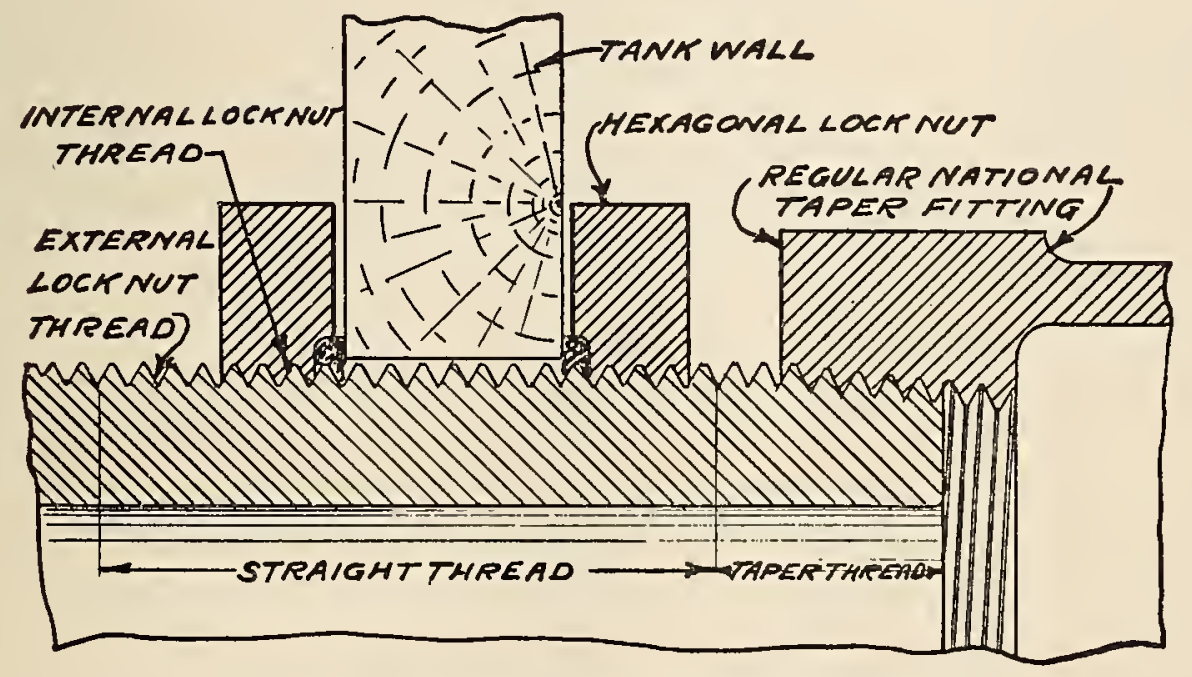

FIG. 31.-Illustration of "tank nipple" thread 


\section{TABLES OF PIPE DIMENSIONS}

Tables $69,70,71$, and 72 , which follow, are not a part of the thread standard, but are reprinted as part of the "Manual on American Standard Pipe Threads."

TABLE 69.-Dimensions of standard wrought pipe

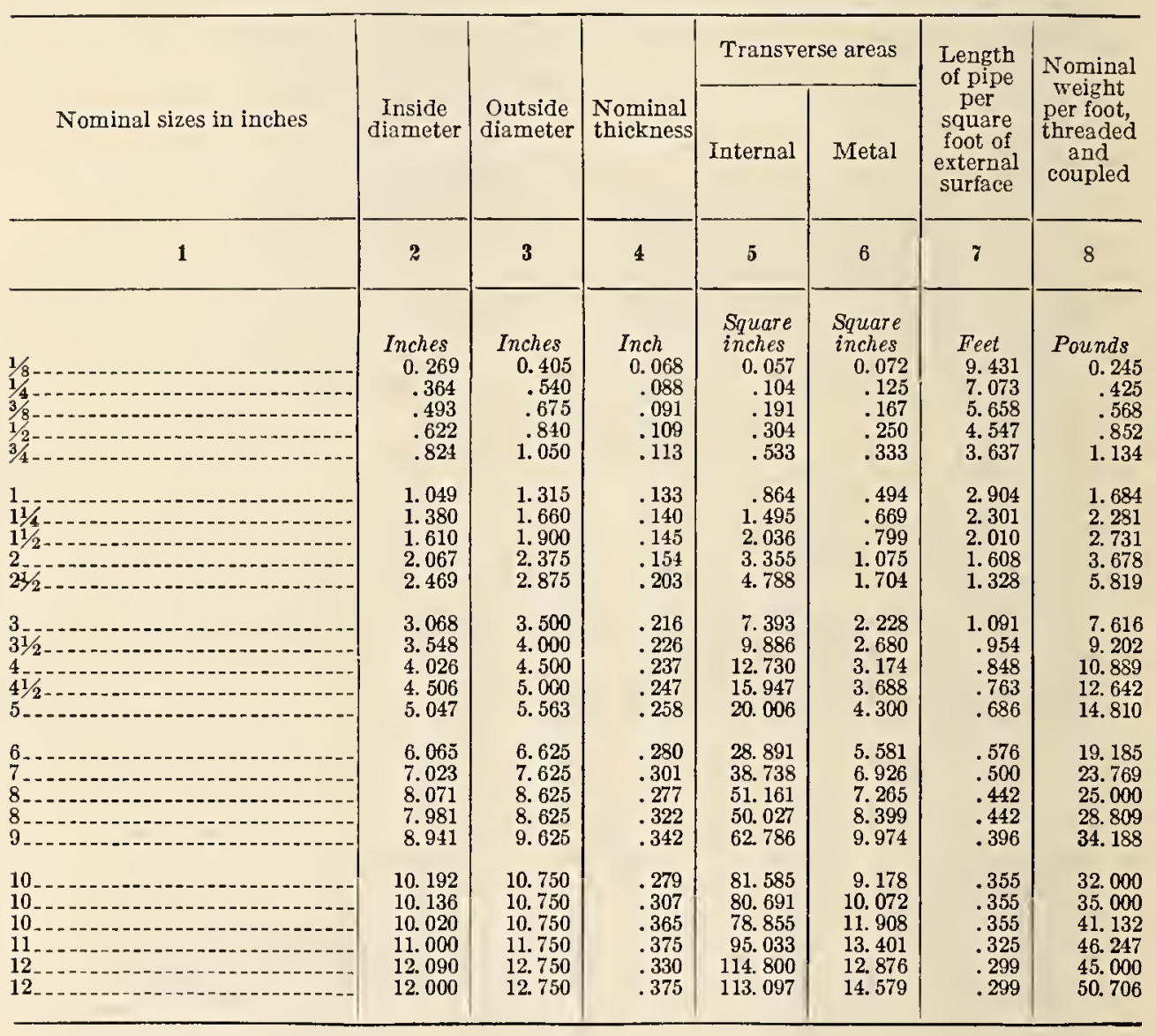


TABLE 70.-Dimensions of extra strong wrought pipe

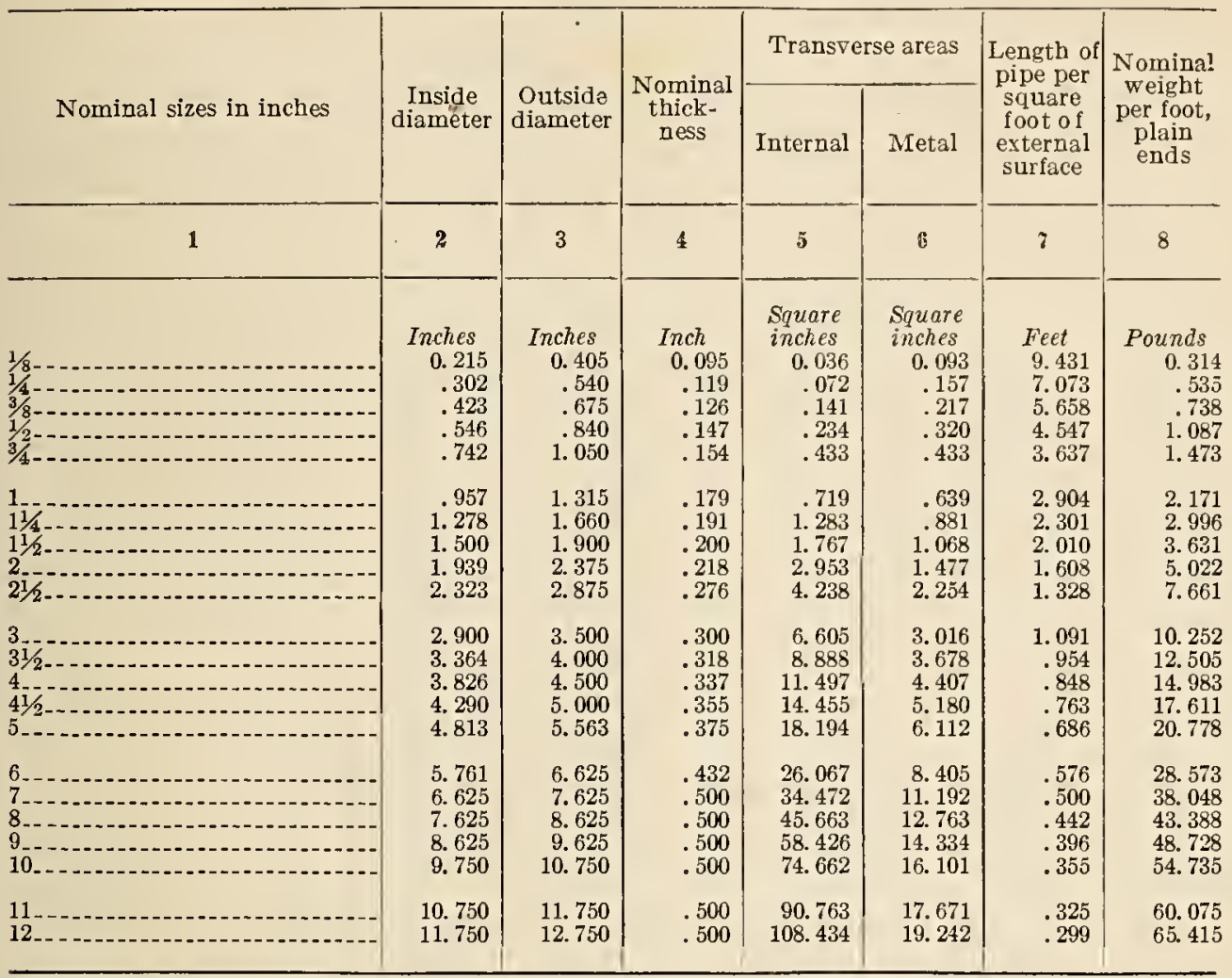

TABLI 71.-Dimensions of double extra strong wrought pipe

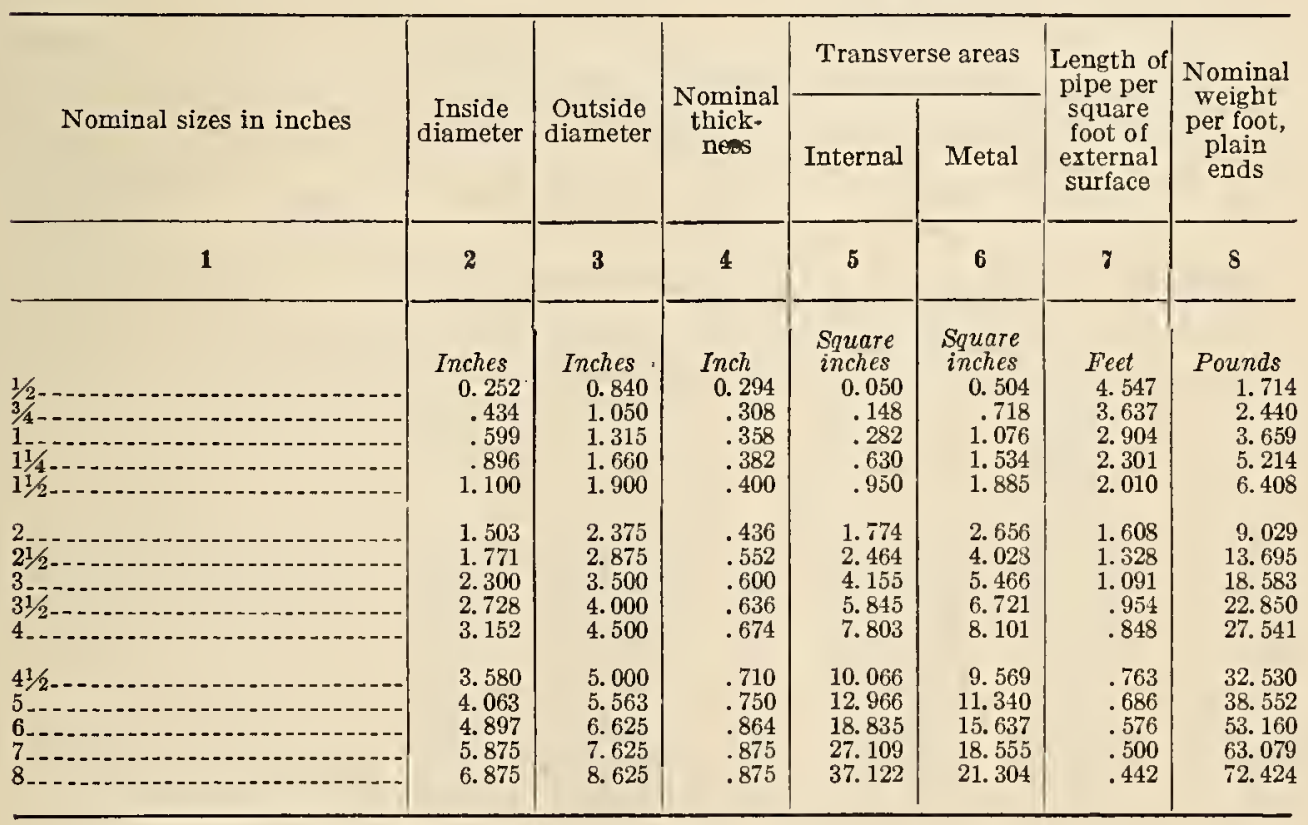

$16802^{\circ}-25 \dagger-9$ 
TABLE 72.-Diameters of large $O . D$. pipe

\begin{tabular}{|c|c|c|c|c|c|c|c|c|c|c|}
\hline \multirow{2}{*}{$\begin{array}{c}\text { Nominal sizes in } \\
\text { inches }\end{array}$} & \multirow{2}{*}{$\begin{array}{l}\text { Out- } \\
\text { side } \\
\text { diam- } \\
\text { eter }\end{array}$} & \multicolumn{9}{|c|}{ Inside diameter } \\
\hline & & $\begin{array}{l}1 / 4 \text { inch } \\
\text { thick }\end{array}$ & $\begin{array}{l}\text { I6 inch } \\
\text { thick }\end{array}$ & $\left|\begin{array}{c}3 / 8 \text { inch } \\
\text { thick }\end{array}\right|$ & $\begin{array}{l}\frac{7}{16} \text { inch } \\
\text { thick }\end{array}$ & $\begin{array}{l}1 / 2 \text { inch } \\
\text { thick }\end{array}$ & $\begin{array}{l}\text { Poinch } \\
\text { thick }\end{array}$ & $\begin{array}{l}5 / 8 \text { inch } \\
\text { thick }\end{array}$ & $\begin{array}{c}3 / 4 \text { inch } \\
\text { thick }\end{array}$ & $\begin{array}{l}1 \text { inch } \\
\text { thick }\end{array}$ \\
\hline 1 & 2 & 3 & 4 & 5 & 6 & $\gamma$ & 8 & 9 & 10 & 11 \\
\hline $\begin{array}{l}14 \\
15 \\
16 \\
18\end{array}$ & $\begin{array}{r}\text { Inches } \\
14 \\
15 \\
16 \\
17 \\
18\end{array}$ & $\begin{array}{r}\text { Inches } \\
131 / 2 \\
141 / 2 \\
151 / 2 \\
161 / 2 \\
171 / 2\end{array}$ & \begin{tabular}{|r|} 
Inches \\
$133 / 8$ \\
$14^{3} / 8$ \\
$15^{3} / 8$ \\
$16^{3} / 8$ \\
$17^{3} / 8$
\end{tabular} & \begin{tabular}{|r|} 
Inches \\
$131 / 4$ \\
$141 / 4$ \\
151 \\
16 \\
$171 / 4$ \\
\end{tabular} & \begin{tabular}{|r|} 
Inches \\
$131 / 8$ \\
$141 / 8$ \\
$151 / 8$ \\
$161 / 8$ \\
$171 / 8$
\end{tabular} & $\begin{array}{r}\text { Inches } \\
13 \\
14 \\
15 \\
16 \\
17\end{array}$ & \begin{tabular}{|r|} 
Inches \\
$127 / 8$ \\
$137 / 8$ \\
1478 \\
$157 / 8$ \\
$167 / 8$
\end{tabular} & \begin{tabular}{|r|} 
Inches \\
$123 / 4$ \\
$133 / 4$ \\
$14^{3} / 4$ \\
$15^{3} / 4$ \\
$16^{3} / 4$
\end{tabular} & \begin{tabular}{|r|} 
Inches \\
$121 / 2$ \\
$131 / 2$ \\
$141 / 2$ \\
$151 / 2$ \\
$161 / 2$
\end{tabular} & $\begin{array}{r}\text { Inches } \\
12 \\
13 \\
14 \\
15 \\
16\end{array}$ \\
\hline $\begin{array}{l}20 \\
22 \\
24 \\
26 \\
30\end{array}$ & $\begin{array}{l}20 \\
22 \\
24 \\
26 \\
28 \\
30\end{array}$ & $\mid$\begin{tabular}{l}
- \\
\hdashline$-\cdots$
\end{tabular} & $\begin{array}{r}193 / 8 \\
213 / 8 \\
\end{array}$ & $\begin{array}{l}191 / 4 \\
211 \\
231 / 4 \\
251 / 4\end{array}$ & $\begin{array}{l}191 / 8 \\
211 / 8 \\
231 / 8 \\
251 / 8 \\
271 / 8 \\
291 / 8\end{array}$ & $\begin{array}{l}19 \\
21 \\
23 \\
25 \\
27 \\
29\end{array}$ & $\begin{array}{l}187 / 8 \\
207 / 8 \\
227 / 8 \\
247 / 8 \\
267 / 8 \\
287 / 8\end{array}$ & $\begin{array}{l}183 / 4 \\
2034 \\
223 / 4 \\
2434 \\
2634 \\
2834\end{array}$ & $\begin{array}{l}181 / 2 \\
201 / 2 \\
221 / 2 \\
241 / 2 \\
261 / 2 \\
281 / 2\end{array}$ & \begin{tabular}{c}
18 \\
$-\cdots-$ \\
\hdashline.- \\
--- \\
$--\cdot$
\end{tabular} \\
\hline
\end{tabular}

\section{THREADING TOOLS}

(a) TAPs.-Recommended dimensions of standard taps for "national taper pipe threads" are given in Table 73.

Recommended dimensions of standard taps for "national straight pipe threads" are given in Table 74 .

(b) TAP DrILLs.-Sizes of tap drills recommended for "national taper pipe threads" are given in Table 75 .

Sizes of tap drills recommended for "national straight pipe threads" are given in Table 76.

TABLE 73.-Dimensions of standard taps for national taper pipe threads

\begin{tabular}{|c|c|c|c|c|c|c|c|c|c|c|}
\hline \multirow{2}{*}{$\begin{array}{l}\text { Nominal sizes } \\
\text { in inches }\end{array}$} & \multicolumn{2}{|c|}{$\begin{array}{l}\text { Length } \\
\text { overall }\end{array}$} & \multicolumn{2}{|c|}{$\begin{array}{l}\text { Length of } \\
\text { thread }\end{array}$} & \multicolumn{2}{|c|}{$\begin{array}{l}\text { Diameter of } \\
\text { shank }\end{array}$} & \multicolumn{2}{|c|}{$\begin{array}{l}\text { Size of } \\
\text { square }\end{array}$} & \multicolumn{2}{|c|}{$\begin{array}{l}\text { Projection of } \\
\text { small end } \\
\text { through } \\
\text { taper gage }\end{array}$} \\
\hline & $\begin{array}{c}\text { Nom- } \\
\text { inal }\end{array}$ & $\begin{array}{l}\text { Toler- } \\
\text { ance }\end{array}$ & $\begin{array}{c}\text { Nom- } \\
\text { inal }\end{array}$ & $\begin{array}{l}\text { Toler- } \\
\text { ance }\end{array}$ & $\begin{array}{l}\text { Nom- } \\
\text { inal } \\
\text { (maxi- } \\
\text { mum) }\end{array}$ & $\begin{array}{l}\text { Toler- } \\
\text { ance }\end{array}$ & $\begin{array}{l}\text { Nom- } \\
\text { inal } \\
\text { (maxi- } \\
\text { mum) }\end{array}$ & $\begin{array}{l}\text { Toler- } \\
\text { ance }\end{array}$ & $\begin{array}{l}\text { Nom- } \\
\text { inal }\end{array}$ & $\begin{array}{l}\text { Toler- } \\
\text { ance }\end{array}$ \\
\hline 1 & 2 & 3 & 4 & 5 & 6 & $\gamma$ & 8 & 9 & 10 & 11 \\
\hline & $\begin{array}{r}\text { Inches } \\
21 / 8 \\
21 / 6 \\
216 \\
31 / 8 \\
31 / 4\end{array}$ & $\begin{array}{c}\text { Inch } \\
\pm \frac{1}{32} \\
\pm \pm \frac{1}{32} \\
\pm \frac{32}{32} \\
\pm \frac{1}{32} \\
\pm \frac{1}{32}\end{array}$ & $\begin{array}{r}\text { Inches } \\
3 / 4 \\
1 \frac{1}{16} \\
1 \frac{1}{15} \\
13 \\
13 / 8\end{array}$ & $\begin{array}{l}\text { Inch } \\
\pm \frac{3}{64} \\
\pm \frac{3}{6} \\
\pm \frac{3}{66} \\
\pm \frac{3}{6} \\
\pm \frac{3}{60}\end{array}$ & $\begin{array}{r}\text { Inches } \\
\mathbf{0 . 4 3 7 5} \\
.5625 \\
.7000 \\
.8650 \\
\mathbf{1 . 0 7 5 0}\end{array}$ & $\begin{array}{c}\text { Inch } \\
-0.007 \\
-.007 \\
-.007 \\
-.007 \\
-.009\end{array}$ & $\begin{array}{r}\text { Inches } \\
0.328 \\
.421 \\
.531 \\
.640 \\
.812\end{array}$ & $\begin{array}{r}\text { Inch } \\
-0.006 \\
-.006 \\
-.006 \\
-.006 \\
-.010\end{array}$ & $\begin{array}{l}\text { Inch } \\
0.312 \\
.459 \\
.454 \\
.579 \\
.565\end{array}$ & $\begin{array}{c}\text { Inch } \\
\pm 0.0625 \\
\pm .0625 \\
\pm .0625 \\
\pm .0625 \\
\pm .0625\end{array}$ \\
\hline & $\begin{array}{l}33 / 4 \\
4 \\
41 / 4 \\
41 / 2 \\
51 / 2\end{array}$ & $\begin{array}{l} \pm \frac{1}{16} \\
\pm 1 \frac{1}{16} \\
\pm \frac{1}{16} \\
\pm \frac{1}{16} \\
\pm \frac{1}{16}\end{array}$ & $\begin{array}{l}13 / 4 \\
13 \\
13 \\
13 \\
134 \\
2 \frac{4}{18}\end{array}$ & $\begin{array}{l} \pm \frac{3}{8} \\
\pm \frac{3}{34} \\
\pm \frac{3}{86} \\
\pm \frac{3}{8} \\
\pm \frac{3}{66}\end{array}$ & $\begin{array}{l}1.1250 \\
1.3125 \\
1.5000 \\
1.8750 \\
2.2500\end{array}$ & $\begin{array}{l}-.009 \\
-.009 \\
-.009 \\
-.009 \\
-.009\end{array}$ & $\begin{array}{r}.843 \\
.984 \\
1.125 \\
1.406 \\
1.687\end{array}$ & $\begin{array}{l}-.010 \\
-.010 \\
-.010 \\
-.010 \\
-.010\end{array}$ & $\begin{array}{l}.678 \\
.686 \\
.699 \\
.667 \\
.925\end{array}$ & $\begin{array}{l} \pm .0938 \\
\pm .0938 \\
\pm .0938 \\
\pm .0938 \\
\pm .0938\end{array}$ \\
\hline & $\begin{array}{l}6 \\
61 / 2 \\
63 / 4\end{array}$ & $\begin{array}{l} \pm \frac{1}{16} \\
\pm \frac{1}{16} \\
\pm \frac{1}{16}\end{array}$ & $\begin{array}{l}25 / 8 \\
214 \\
23 / 4\end{array}$ & $\begin{array}{l} \pm \frac{3}{67} \\
\pm \frac{3}{62} \\
\pm \frac{3}{64}\end{array}$ & $\begin{array}{l}2.6250 \\
2.8125 \\
\text { 3. } 0000\end{array}$ & $\begin{array}{l}-.009 \\
-.009 \\
-.009\end{array}$ & $\begin{array}{l}1.968 \\
2.108 \\
2.250\end{array}$ & $\begin{array}{r}-.010 \\
-.010 \\
-.010\end{array}$ & $\begin{array}{r}.925 \\
.938 \\
.950\end{array}$ & $\begin{array}{l} \pm .0938 \\
\pm .1250 \\
\pm .1250\end{array}$ \\
\hline
\end{tabular}


TABLE 74.-Dimensions of standard taps for national straight pipe threads

\begin{tabular}{|c|c|c|c|c|c|c|c|c|c|c|c|c|}
\hline \multirow{2}{*}{$\begin{array}{c}\text { Nominal } \\
\text { sizes in } \\
\text { inches }\end{array}$} & \multicolumn{2}{|c|}{$\begin{array}{l}\text { Length } \\
\text { overall }\end{array}$} & \multicolumn{2}{|c|}{$\begin{array}{l}\text { Length of } \\
\text { thread }\end{array}$} & \multicolumn{2}{|c|}{$\begin{array}{l}\text { Diameter of } \\
\text { shank }\end{array}$} & \multicolumn{2}{|c|}{$\begin{array}{l}\text { Size of } \\
\text { square }\end{array}$} & \multicolumn{3}{|c|}{ Pitch diameter } & \multirow{2}{*}{$\begin{array}{c}\begin{array}{c}\text { Major } \\
\text { diam- } \\
\text { eter }\end{array} \\
\begin{array}{c}\text { Nom- } \\
\text { inal }\end{array}\end{array}$} \\
\hline & $\begin{array}{c}\text { Nom- } \\
\text { inal }\end{array}$ & $\begin{array}{l}\text { Toler- } \\
\text { ance }\end{array}$ & $\begin{array}{c}\text { Nom- } \\
\text { inal }\end{array}$ & $\begin{array}{l}\text { Toler- } \\
\text { ancc }\end{array}$ & $\begin{array}{l}\text { Nom- } \\
\text { inal } \\
\text { (maxi- } \\
\text { mum) }\end{array}$ & $\begin{array}{l}\text { Toler- } \\
\text { ance }\end{array}$ & $\begin{array}{l}\text { Nom- } \\
\text { inal } \\
\text { (maxi- } \\
\text { mum) }\end{array}$ & $\begin{array}{l}\text { Toler- } \\
\text { ance }\end{array}$ & $\begin{array}{l}\text { Maxi- } \\
\text { mum }\end{array}$ & Basic & $\begin{array}{l}\text { Mini- } \\
\text { mum }\end{array}$ & \\
\hline 1 & 2 & 3 & 4 & $\tilde{E}$ & 6 & $y$ & 8 & 9 & 10 & 11 & 12 & 13 \\
\hline & $\begin{array}{r}\text { Inches } \\
21 / 8 \\
2 \frac{7}{16} \\
218 \\
31 / 8 \\
31 / 4\end{array}$ & $\begin{array}{l}\text { Inch } \\
\pm \frac{1}{32} \\
\pm \frac{2}{32} \\
\pm \frac{2}{32} \\
\pm \frac{12}{32} \\
\pm \frac{12}{32}\end{array}$ & $\begin{array}{c}\text { Inches } \\
3 / 4 \\
1 \frac{1}{18} \\
1 \frac{1}{18} \\
13 \\
13 / 8\end{array}$ & $\begin{array}{c}\text { Inch } \\
\pm \frac{3}{66} \\
\pm \frac{3}{86} \\
\pm \frac{8}{66} \\
\pm \frac{36}{66} \\
\pm \frac{3}{66}\end{array}$ & $\begin{array}{r}\text { Inches } \\
0.4375 \\
.5625 \\
.7000 \\
.8650 \\
1.0750\end{array}$ & $\begin{array}{r}\text { Inch } \\
-0.007 \\
-.007 \\
-.007 \\
-.007 \\
-.009\end{array}$ & $\begin{array}{c}\text { Inches } \\
0.328 \\
.421 \\
.531 \\
.640 \\
.812\end{array}$ & $\begin{array}{c}\text { Inch } \\
-0.006 \\
-.006 \\
-.006 \\
=.006 \\
-.010\end{array}$ & $\begin{array}{r}\text { Inches } \\
0.3763 \\
.4914 \\
.6288 \\
.7802 \\
.9909\end{array}$ & $\begin{array}{r}\text { Inches } \\
0.3743 \\
.4899 \\
.6270 \\
.7784 \\
.9889\end{array}$ & $\begin{array}{r}\text { Inches } \\
0.3733 \\
.4884 \\
.6253 \\
.7767 \\
.9869\end{array}$ & $\begin{array}{l}\text { Inches } \\
0.4044 \\
.5343 \\
.6715 \\
.8356 \\
1.0460\end{array}$ \\
\hline & $\begin{array}{l}33 / 4 \\
4 \\
41 / 4 \\
41 / 2 \\
51 / 2\end{array}$ & $\begin{array}{l} \pm \frac{1}{18} \\
\pm \frac{2}{16} \\
1 \frac{1}{18} \\
\pm \frac{1}{16} \\
\pm \frac{1}{16}\end{array}$ & $\begin{array}{l}13 / 4 \\
13 / 4 \\
13 / 4 \\
13 / 4 \\
2 \frac{9}{16}\end{array}$ & $\begin{array}{l} \pm \frac{3}{66} \\
\pm \frac{3}{66} \\
\pm \frac{3}{64} \\
\pm \frac{3}{63} \\
\pm \frac{3}{66}\end{array}$ & $\begin{array}{l}1.1250 \\
1.3125 \\
1.5000 \\
1.8750 \\
2.2500\end{array}$ & $\begin{array}{r}-.009 \\
-.009 \\
-.009 \\
-.009 \\
-.009\end{array}$ & $\begin{array}{r}.843 \\
.984 \\
1.125 \\
1.466 \\
1.687\end{array}$ & $\begin{array}{l}-.010 \\
-.010 \\
-.010 \\
-.010 \\
-.010\end{array}$ & $\begin{array}{l}1.2406 \\
\text { 1. } 5856 \\
\text { 1. } 8246 \\
\text { 2. } 2988 \\
\text { 2. } 7649\end{array}$ & $\begin{array}{l}\text { 1. } 2386 \\
1.5834 \\
1.8223 \\
2.2963 \\
\text { 2. } 7622\end{array}$ & $\begin{array}{l}\text { 1. } 2366 \\
1.5811 \\
1.8201 \\
2.2938 \\
2.7594\end{array}$ & $\begin{array}{l}\text { 1. } 3082 \\
1.6529 \\
1.8919 \\
2.3658 \\
2.8622\end{array}$ \\
\hline $\begin{array}{l}3 . \\
31 \\
4.2\end{array}$ & $\begin{array}{l}6 \\
61 / 2 \\
6^{3} / 4\end{array}$ & $\begin{array}{l} \pm \frac{1}{16} \\
\pm \frac{8}{10} \\
\pm \frac{1}{16}\end{array}$ & $\begin{array}{l}25 / 8 \\
211 \\
23 / 4\end{array}$ & $\begin{array}{l} \pm \frac{3}{66} \\
\pm \frac{3}{64} \\
\pm \frac{3}{68}\end{array}$ & $\begin{array}{l}2.6250 \\
2.8125 \\
3.0000\end{array}$ & $\begin{array}{l}-.009 \\
-.009 \\
-.009\end{array}$ & $\begin{array}{l}\text { 1. } 968 \\
\text { 2. } 108 \\
2.250\end{array}$ & $\begin{array}{l}-.010 \\
-.010 \\
-.010\end{array}$ & $\begin{array}{l}3.3913 \\
3.8916 \\
4.3899\end{array}$ & $\begin{array}{l}3.3885 \\
\text { 3. } 8888 \\
4.3871\end{array}$ & $\begin{array}{l}\text { 3. } 3858 \\
3.8861 \\
4.3844\end{array}$ & $\begin{array}{l}3.4885 \\
3.9888 \\
4.4871\end{array}$ \\
\hline
\end{tabular}

TABLE 75.-Sizes of tap drills, national taper pipe threads

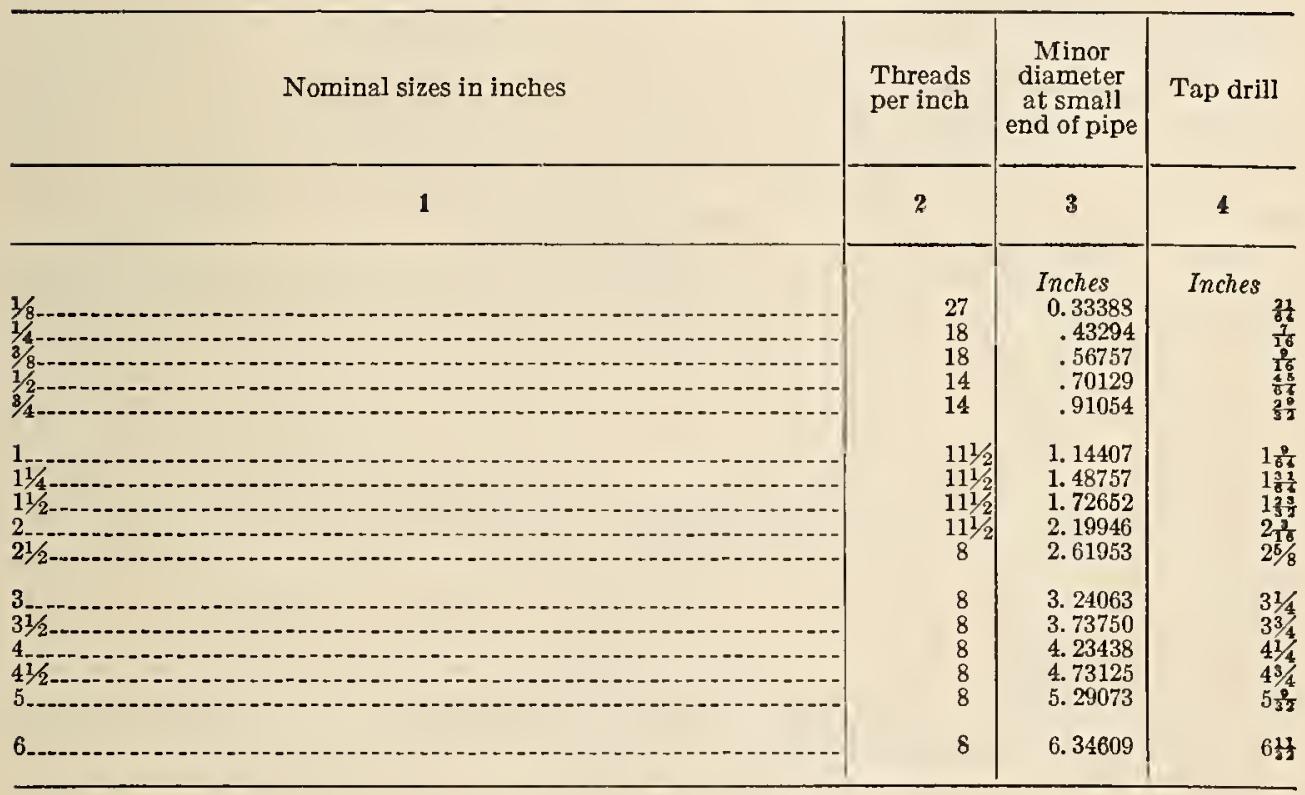


TABLE 76.-Sizes of tap drills, national straight pipe threads

\begin{tabular}{|c|c|c|c|}
\hline Nominal sizes in inches & $\begin{array}{l}\text { Threads } \\
\text { per inch }\end{array}$ & $\begin{array}{l}\text { Minor } \\
\text { diameter, } \\
\text { basic }\end{array}$ & Tap drill \\
\hline 1 & 2 & 3 & 4 \\
\hline 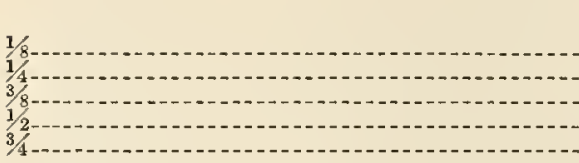 & $\begin{array}{l}27 \\
18 \\
18 \\
14 \\
14\end{array}$ & $\begin{array}{l}\text { Inches } \\
0.34513 \\
.44544 \\
.58257 \\
.72129 \\
.93172\end{array}$ & 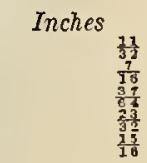 \\
\hline 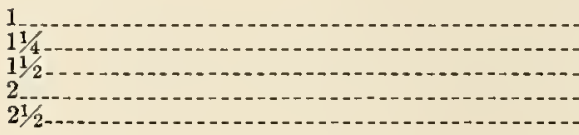 & $\begin{array}{l}111 / 2 \\
111 / 2 \\
111 / 2 \\
111 / 2 \\
8\end{array}$ & $\begin{array}{l}\text { 1. } 16907 \\
\text { 1. } 51382 \\
1.75277 \\
2.22671 \\
2.66216\end{array}$ & $\begin{array}{l}1 \frac{5}{38} \\
1 \frac{3}{64} \\
13 \frac{1}{3} \\
2 \frac{7}{32} \\
2 \frac{32}{32}\end{array}$ \\
\hline $44^{3}{ }^{3} 1 / 2-2$ & $\begin{array}{l}8 \\
8 \\
8 \\
8 \\
8\end{array}$ & $\begin{array}{l}\text { 3. } 28850 \\
\text { 3. } 78881 \\
\text { 4. } 28713 \\
\text { 4. } 78594 \\
\text { 5. } 34929\end{array}$ & $\begin{array}{l}39 \\
3 \frac{9}{35} \\
4 \frac{9}{32} \\
4 \frac{35}{32} \\
5 \frac{12}{32}\end{array}$ \\
\hline 6 & 8 & 6. 40597 & $6 \frac{13}{32}$ \\
\hline
\end{tabular}

\section{GAGES}

In order properly to maintain interchangeability of pipe threads, gages should consist of "master," "check" or "setting," "inspection," and "working" gages. The same fundamentals apply as those outlined in Section III covering gages for fastening screws, with the single exception that, with taper threaded gages, separate "go" and "not go" gages are not necessary.

(a) Classification of Gages.-1. Master gage.-The master gage is a taper threaded plug gage. The roots of the threads are cut to a sharp $\mathrm{V}$ or may be undercut below the sharp $\mathrm{V}$ to facilitate the making of the thread. The crests are truncated an amount equal to $0.1 p$ (see fig. 32). ${ }^{14}$ Basic dimensions of taper pipe thread gages are given in Table 81 . This gage is provided with the gaging notch as illustrated in Figure 32 . The master gage is the gage to which all other gages are ultimately referred either by transfer of measurements or direct comparison by engagement. It is intended primarily for the use of gage and thread tool manufacturers.

2. Check (or reference) gages.-The check gages consist of a plug gage, similar in all respects to the master gage, and one ring gage. The ring gage has a thickness equal to dimension $L_{1}$, is the same diameter at the small end as the small end of the plug gage, and is flush with the plug gage at the small end and at the gaging notch when screwed on tight by hand (see fig. 32). The check plug gage is used to inspect inspection and working taper threaded ring gages. The check ring gage is used to compare the check plug with the

${ }^{34}$ The object of truncating the crests on gages (truncation $0.1 p$ ) is to insure that, when gaging commercial threads cut with a slightly dull tool, the gage bears on the sides of the thread instead of on the roots. 
master plug, or the inspection and working plug gages with the check plug gage.

3. Inspection gages.-Inspection gages consist of one taper threaded plug gage and one taper threaded ring gage. The roots of the threads are cut to a sharp $\vee$ or may be undercut to facilitate making the thread. The crests are truncated an amount equal to $0.1 p$, but otherwise the gages are made to the dimensions given in Table $66{ }^{14}$ The ring gage has a thickness equal to dimension $L_{1}$, and the same diameter at the small end as the small end of the plug gage (see fig. 33).

Inspection gages are for the use of the purchaser of pipe thread products. When used, the extreme tolerances on the work should be applied. This tolerance is one and one-half turns either way from the gaging notch in the case of internal threads inspected with

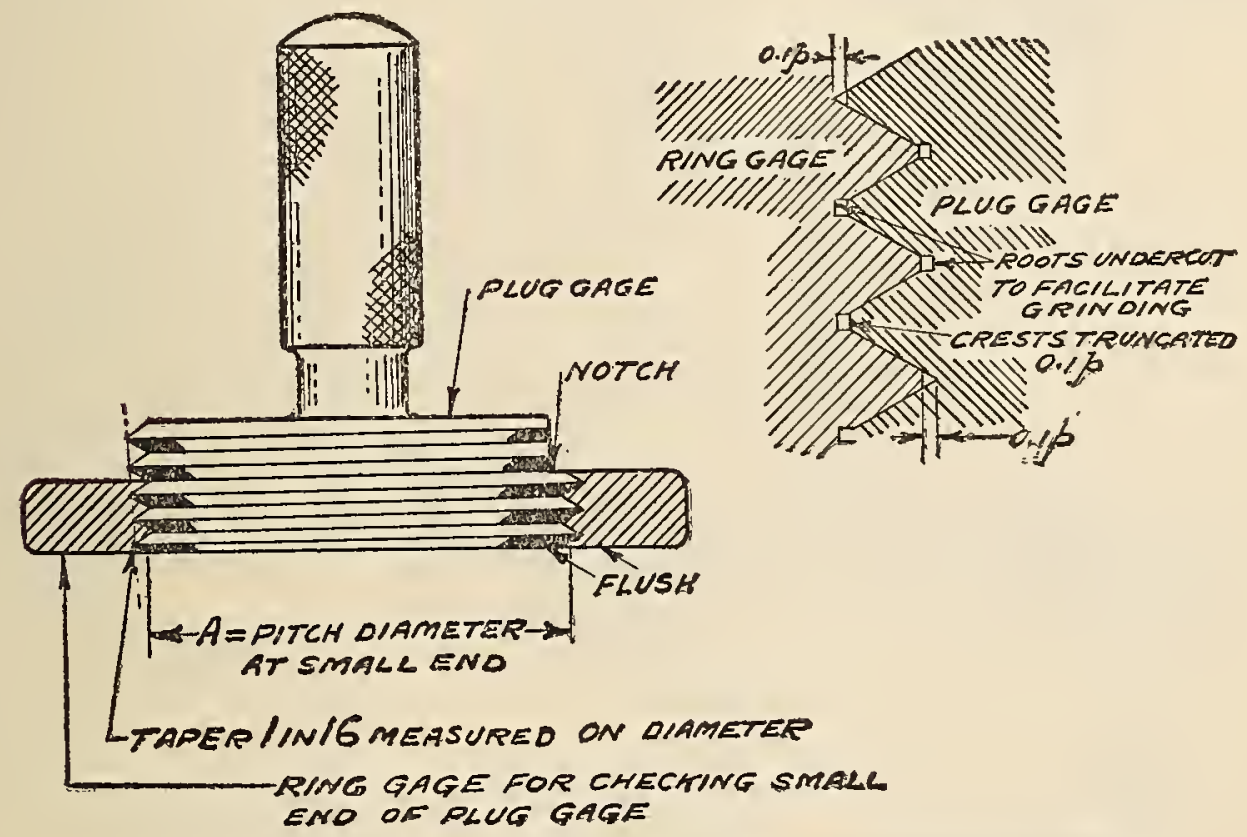

FIG. 32.-Master gages or check gages for checking working gages

the inspection plug gage, and when inspecting external threads the tolerance is one and one-half turns either way from the small end of the inspection ring. Inspection gages should be checked frequently against the check gages, and in use their errors should be taken into account.

4. Working gages. - The working gages consist of one taper threaded plug and one taper threaded ring gage. These gages are similar in all respects to the inspection plug and ring gages. The working gages are used by the manufacturer to inspect his product. In using the working gages, the tolerance to be applied is one turn either way from the gaging notch in case of internal threads inspected with the plug 
gage, and in the case of external threads the tolerance is one turn either way from the small end of the working ring gage.

(b) Gaging Practices.-1. Gaging internal threads.-The inspection and working plug gages, Figure 34 , should screw tight by hand into the fitting or coupling until the notch is flush with the face. When the thread is chamfered, the notch should be flush with the bottom of the chamfer. The fitting or coupling is within the working or net tolerance if the working gage notch is within one turn of the coupling or fitting face when screved in tight by hand. In the same way the coupling or fitting is within the inspection or extreme tolerance if the inspection gage notch is within one and one-half turns of the coupling or fitting when screwed on tight by hand.

This method of gaging is used either for taper internal threads or for straight internally threaded couplings which screw together with taper external threads.

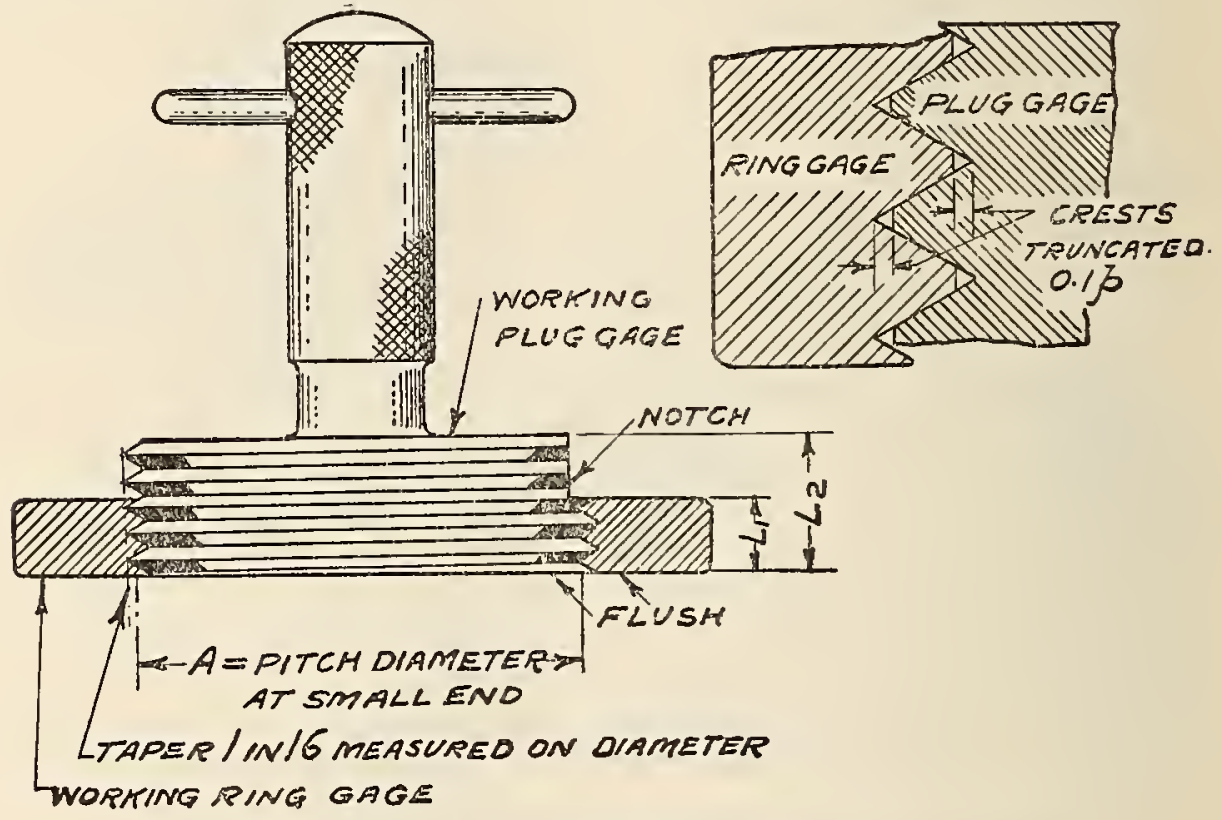

FIG. 33.-Inspection or working gages for checking product

2. Gaging taper external threads.-The ring gage, Figure 35, should screw tight by hand on the pipe or external thread until the small end of the gage is flush with the end of the thread. The pipe or external thread is within the working or net tolerance if the working ring gage screws on until the end of pipe or external thread is within one turn of the small end of the gage. The pipe or external thread is within the inspection or extreme tolerance if the inspection ring screws on until the end of pipe is within one and one-half turns of the small end of the gage.

(c) Specifications for Gages.-1. Master gages.-Master gages shall be made within the narrowest possible limits of error. In no 
case should the accumulative error exceed the total accumulative tolerance on diameter given in Table 77. Each master gage shall be accompanied by a report showing the error on each of the elements of thread and a statement of the accumulative error derived from the errors in the various elements. In case of question, the deviations of this gage from the basic size shall be ascertained by the Bureau of Standards at Washington, D. C.
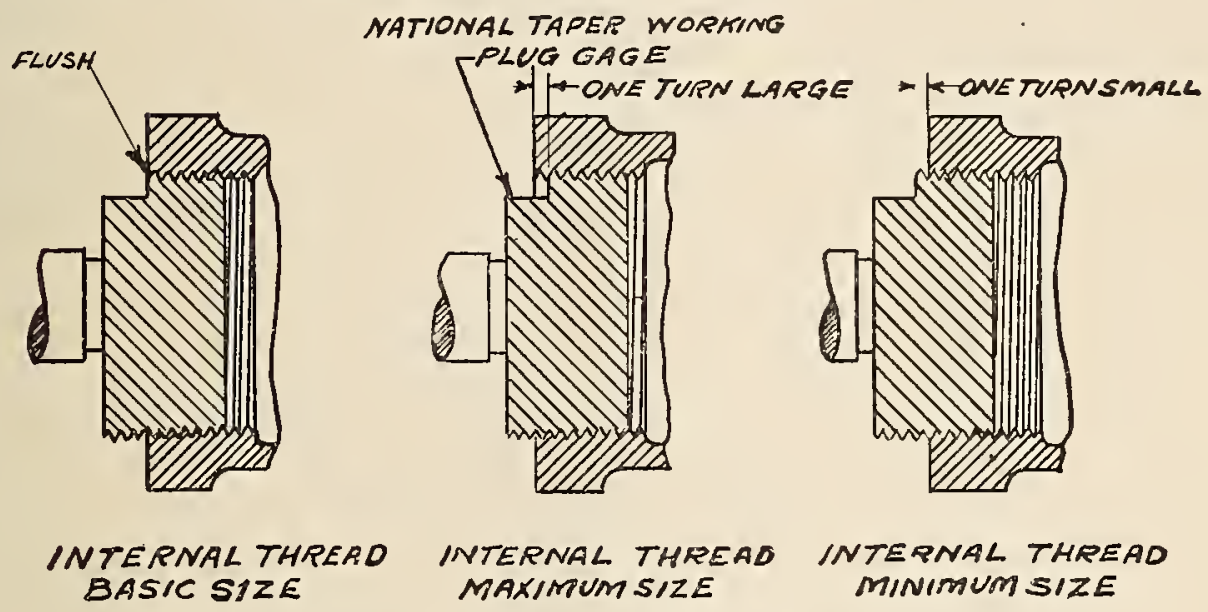

INTERNAL THRERO INTERNAL THREAD INTERNAL THREAD

FIG. 34.-Gaging of internal national taper pipe threads

2. Check (or reference) gages.-Column 2 of Table 77 gives the maximum allowable accumulation of all errors in the thread surface of a check gage, expressed in terms of diameter, as illustrated in Figure 36. No point on the thread surface of the gage should be outside of the zone of tolerance indicated by the shaded portion of

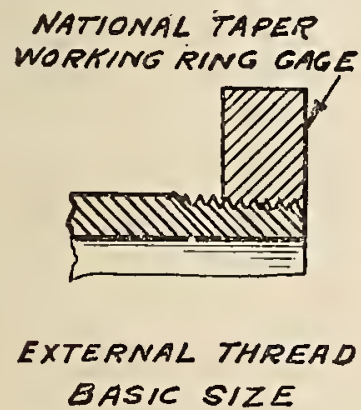

BASIC SIZE

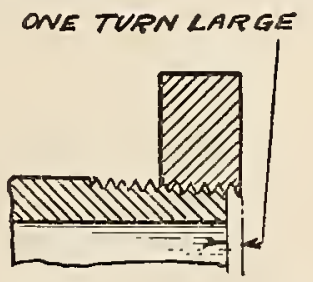

EXTERNAL THREAD MAXIMUM SIZE

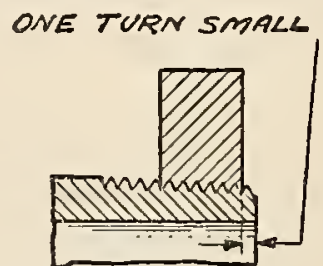

EXTERNAL THREAD MINIMUM SIZE

FIG. 35.-Gaging of external national taper pipe threads

the illustration. This column is used when checking gages by measurement. If the errors in the gage are reported in terms of pitch, angle of thread, and diameter, Tables 79 and 80 may be used to determine the accumulation of these errors for comparison with column 2. In Table 79 the results of errors in angle are expressed in terms of diameter. In Table 80 the results of errors in pitch are expressed in terms of diameter. 
For example: A three-fourths inch, 14 thread, pipe thread plug gage is reported as follows:

Pitch diameter, large end, 0.98881 inch.

Pitch diameter, small end, 0.96775 inch.

One-half included angle of thread, $29^{\circ} 58^{\prime}$.

Maximum error in lead, 0.00007 inch.

The correct pitch diameter at large end is 0.98886 inch. The error is 0.00005 inch. The correct pitch diameter at small end is 0.96768 inch. (See Table 66.)

Error of $2^{\prime}$ in angle is equivalent to 0.00005 inch. 0.00007 inch error in lead is equivalent to 0.00012 inch. (See Table 66.) The accumulative error at large end in terms of diameter $=0.00023$ inch. The accumulative error at small end equals 0.00025 inch.

The gage falls within the limits of the check gage $(0.00028$ inch as given in Table 77).

Column 3 of Table 77 gives the equivalent of column 2, expressed in terms of distance parallel to the axis, and represents the maximum distance which a check ring gage of perfect thickness, or a check plug gage of perfect length from small end to gaging notch, may vary from being flush at the gaging notch, or at the small end, when referred to basic dimensions. It is equal to 16 times column 2 because of the basic taper of 1 in 16, measured on the diameter. This column is used when checking check gages by comparison with a master gage. The necessary allowance must be made for the error in the master gage.

Column 4 of Table 77, gives the equivalent of column 3, expressed in terms of the decimal part of a turn. This column is also used when checking check gages by comparison with a master gage. The necessary allowance must be made for the error in the master gage.

A tolerance of plus or minus 0.0002 inch is allowed on the distance between the gaging notch and the small end of the check plug gage, or on the thickness of the check ring gage.

It is possible for check plug and ring gages, which come within all of the above tolerances, to vary from being flush with each other at the small end, or at the gaging notch, when scrcwed together tight by hand. The maximum variation which might occur, expressed in terms of distance, is given in column 5 of Table 77, and gages which come within these limits should be checked by measurement before being rejected.

3. Inspection gages.-The tolerances on new inspection gages are the same as on working gages. (See Table 78.)

4. New working gages. - Column 2 of Table 78 gives the maximum allowable accumulation of all errors in the thread surface of new working gages, expressed in terms of diameter, as illustrated in Figure 36. No point in the thread surface of the gage should be 
outside of the zone of tolerance indicated by the shaded portion of the illustration. This column is used when checking gages by measurement.

Column 3 of Table 78 gives the equivalent of column 2, expressed in terms of distance parallel to the axis, and represents the maximum distance which a new working ring gage of perfect thickness, or a new working plug gage of perfect length from small end to gaging notch, may vary from being flush at the gaging notch, or at the small end, when referred to basic dimensions. It is equal to 16 times column 2, because of the basic taper of 1 in 16, measured on the diameter. This column is used when checking working gages by comparison with a gage, the error of which is known. The necessary allowance must be made for this error.

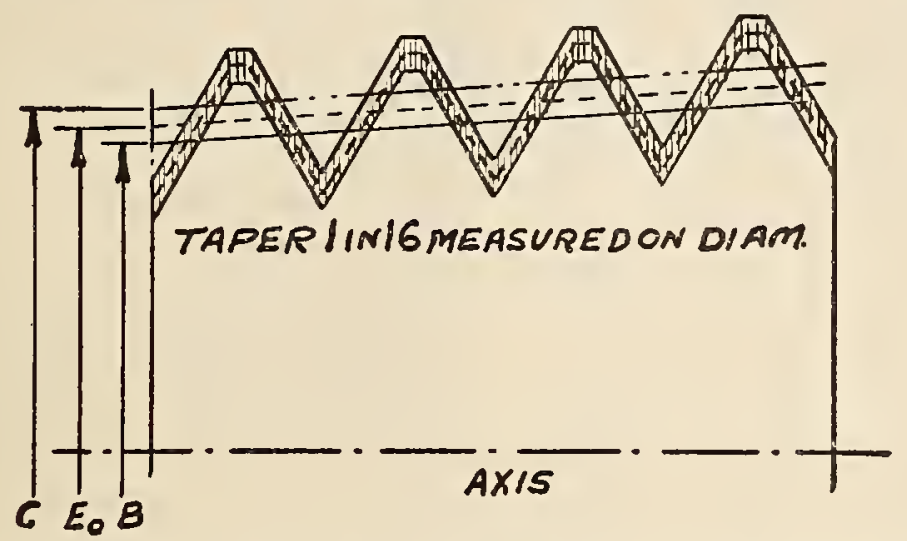

Fig. 36.--Relation of tolerance to basic dimensions of a correct taper pipe thread gage

$E_{0}=$ basic pitch diameter at small end of gage

$B=$ minimum pitch diameter at small end of gage

$C=$ maximum pitch diameter at small end of gage

$B=E_{\mathrm{o}}-$ column 2 from Table 77 for check gages, or column 2 from Table 78 for new working gages

$C=E_{0}+$ column 2 from Table 77 for check gages, or column 2 from Table 78 for new working gages

Note.-No point of the thread surface of the gage should be outside of the zone of tolerance indicated by the shaded portion of the illustration. The dotted line indicates the outline of a perfect gage made exactly to the basic dimensions.

Column 4 of Table 78 gives the equivalent of column 3, expressed in terms of the decimal part of a turn. This column is also used when checking working gages by comparison with a gage, the error of which is known. The necessary allowance imust be made for this error.

A tolerance of plus or minus 0.0005 inch is allowed on the distance between the gaging notch and the small end of the working plug gage, or on the thickness of the working ring gage.

It is possible for working plug and ring gages which come within all of the above tolerances to vary from being flush with each other at the small end or at the gaging notch, when screwed together tight by hand. The maximum variation which might occur, expressed in terms of distance, is given in column 5 of Table 78, and gages which come within these limits should be checked by comparison with reference gages before being rejected. 
It is also possible for working plug and ring gages which come within all of the above tolerances to vary from being flush at the small end or at the gaging notch, when screwed tight by hand on a reference gage which comes within the tolerances specified for reference gages. The maximum variation which might occur, expressed in terms of distance, is given in column 6 of Table 78, and gages which come within these limits should be checked by measurement before being rejected.

5. Worn working gages.-The maximum wear on working gages must not be more than the equivalent of one-half turn from the basic dimensions.

In order that no work passed by the working gage shall be rejected by the inspection gage, it will be necessary to discontinue the use of the working gage when it has worn one-half turn; that is, the working gage should always be kept within the tolerance equivalent of one-half turn from the basic dimensions.

TABLE 77.-Tolerances for chech (or reference) gages, national taper pipe threads

\begin{tabular}{|c|c|c|c|c|}
\hline Nominal sizes in inches & $\begin{array}{l}\text { Total ac- } \\
\text { cumulatice } \\
\text { tolerance } \\
\text { on Jiam- } \\
\text { eter (see } \\
\text { fig. } 36)\end{array}$ & $\begin{array}{l}\text { Equivalent } \\
\text { longitudi- } \\
\text { nal varia- } \\
\text { tion }(16 \times \\
\text { column } 2)\end{array}$ & $\begin{array}{c}\text { Equivalent } \\
\text { angular } \\
\text { variation } \\
\text { cxpressed } \\
\text { as decimal } \\
\text { part of one } \\
\text { turn }\end{array}$ & (l) \\
\hline 1 & 2 & 3 & 4 & 5 \\
\hline $\begin{array}{l}1 / 8 \\
1 / 8 \\
1 / 48 \\
1 / 2 \\
3 / 4-2\end{array}$ & $\begin{array}{l}\text { Inch } \\
0.00020 \\
.00022 \\
.00024 \\
.00026 \\
.00028\end{array}$ & $\begin{array}{r}\text { Inch } \\
0.0032 \\
.0035 \\
.0038 \\
.0042 \\
.0045\end{array}$ & $\begin{array}{l}0.086 \\
.063 \\
.068 \\
.059 \\
.063\end{array}$ & $\begin{array}{r}\text { Inch } \\
0.0068 \\
.0074 \\
.0080 \\
.0088 \\
.0094\end{array}$ \\
\hline $\begin{array}{l}1 \\
1 \\
1\end{array}$ & $\begin{array}{l}.00030 \\
.00032 \\
.00034 \\
.00036 \\
.00038\end{array}$ & $\begin{array}{l}.0048 \\
.0051 \\
.0054 \\
.0058 \\
.0061\end{array}$ & $\begin{array}{l}.055 \\
.059 \\
.062 \\
.067 \\
.050\end{array}$ & $\begin{array}{l}.0100 \\
.0106 \\
.0112 \\
.0120 \\
.0126\end{array}$ \\
\hline $\begin{array}{l}31 / 2 \\
41 / 2-20\end{array}$ & $\begin{array}{l}.00038 \\
.00041 \\
.00043 \\
.00045 \\
.00047\end{array}$ & $\begin{array}{l}.0061 \\
.0066 \\
.0069 \\
.0072 \\
.0075\end{array}$ & $\begin{array}{l}.050 \\
.053 \\
.055 \\
.058 \\
.060\end{array}$ & $\begin{array}{l}.0126 \\
.0136 \\
.0142 \\
.0148 \\
.0154\end{array}$ \\
\hline $\begin{array}{l}6 \\
6 \\
7_{1}\end{array}$ & $\begin{array}{l}.00051 \\
.00055 \\
.00059 \\
.00063 \\
.00066\end{array}$ & $\begin{array}{l}.0082 \\
.0088 \\
.0094 \\
.0101 \\
.0106\end{array}$ & $\begin{array}{l}.065 \\
.070 \\
.075 \\
.080 \\
.085\end{array}$ & $\begin{array}{l}.0168 \\
.0180 \\
.0192 \\
.0206 \\
.0216\end{array}$ \\
\hline $11{ }^{12} 1{ }^{1}$ & $\begin{array}{r}00070 \\
.00074 \\
.00082 \\
.00090 \\
.00098\end{array}$ & $\begin{array}{l}.0112 \\
.0118 \\
.0131 \\
.0144 \\
.0157\end{array}$ & $\begin{array}{l}.090 \\
.095 \\
.105 \\
.115 \\
.125\end{array}$ & $\begin{array}{r}.0228 \\
.0240 \\
.0266 \\
.0292 \\
0318\end{array}$ \\
\hline 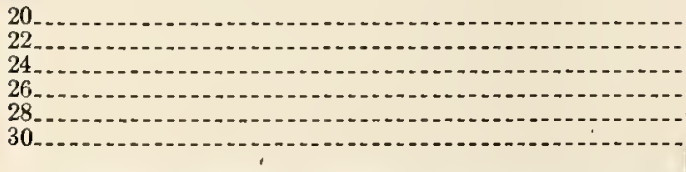 & $\begin{array}{l}.00106 \\
.00113 \\
.00121 \\
.00129 \\
.00137 \\
.00144\end{array}$ & $\begin{array}{l}.0170 \\
.0181 \\
.0194 \\
.0206 \\
.0219 \\
.0230\end{array}$ & $\begin{array}{l}135 \\
.145 \\
.155 \\
.165 \\
.175 \\
.185\end{array}$ & $\begin{array}{l}.0344 \\
.0366 \\
.0392 \\
.0416 \\
.0442 \\
.0464\end{array}$ \\
\hline
\end{tabular}

1 Maximum amount it is possible for plug and ring gages to vary from being flush at small end or at gag. ing notch when screwed together tight by hand ( 2 times column $3+0.0004$ inch). 
TABLE 78.-Tolerances for inspection and working gages, national (American Briggs') taper pipe threads

\begin{tabular}{|c|c|c|c|c|c|}
\hline Nominal sizes in inches & $\begin{array}{c}\text { Total ac- } \\
\text { cumulative } \\
\text { tolerance } \\
\text { on diameter } \\
\text { (see fig. 36) }\end{array}$ & $\begin{array}{l}\text { Equiva- } \\
\text { lent longi- } \\
\text { tudinal } \\
\text { variation } \\
(16 \times \\
\text { column 2) }\end{array}$ & (l) & (2) & $\left({ }^{3}\right)$ \\
\hline \multirow[t]{2}{*}{1} & 2 & 3 & 4 & 5 & 6 \\
\hline & $\begin{array}{l}\text { Inch } \\
0.00040 \\
.00044 \\
.00048 \\
.00052 \\
.00056\end{array}$ & $\begin{array}{c}\text { Inch } \\
0.0061 \\
.0070 \\
.0077 \\
.0083 \\
.0090\end{array}$ & $\begin{array}{r}0.172 \\
.126 \\
.136 \\
.118 \\
.126\end{array}$ & $\begin{array}{r}\text { Inch } \\
0.0138 \\
.0150 \\
.0164 \\
.0176 \\
.0190\end{array}$ & $\begin{array}{r}\text { Inch } \\
0.0103 \\
.0112 \\
.0122 \\
.0132 \\
.0142\end{array}$ \\
\hline $\begin{array}{l}1 \\
11 / 4 / 2 \\
11 / 2 \\
2 \\
21 / 2-200\end{array}$ & $\begin{array}{l}.00060 \\
.00064 \\
.00068 \\
.00072 \\
.00076\end{array}$ & $\begin{array}{l}.0096 \\
.0102 \\
.0109 \\
.0115 \\
.0122\end{array}$ & $\begin{array}{l}.110 \\
.118 \\
.124 \\
.134 \\
.100\end{array}$ & $\begin{array}{l}.0202 \\
.0214 \\
.0228 \\
.0240 \\
.0254\end{array}$ & $\begin{array}{l}.0151 \\
.0160 \\
.0170 \\
.0180 \\
.0190\end{array}$ \\
\hline $\begin{array}{l}3 \\
31 \\
4 \\
4 \\
4 \\
5\end{array}$ & $\begin{array}{l}.00076 \\
.00082 \\
.00086 \\
.00090 \\
.00094\end{array}$ & $\begin{array}{l}.0122 \\
.0131 \\
.0138 \\
.0144 \\
.0150\end{array}$ & $\begin{array}{l}.100 \\
.105 \\
.110 \\
.115 \\
.120\end{array}$ & $\begin{array}{l}.0254 \\
.0272 \\
.0286 \\
.0298 \\
.0310\end{array}$ & $\begin{array}{r}.0190 \\
.0204 \\
.0214 \\
.0223 \\
.0232\end{array}$ \\
\hline $\begin{array}{l}6 \\
6\end{array}$ & $\begin{array}{l}.00102 \\
.00110 \\
.00118 \\
.00126 \\
.00132\end{array}$ & $\begin{array}{l}.0163 \\
.0176 \\
.0189 \\
.0202 \\
.0211\end{array}$ & $\begin{array}{l}130 \\
140 \\
150 \\
.160 \\
170\end{array}$ & $\begin{array}{l}.0336 \\
.0362 \\
.0388 \\
.0414 \\
.0432\end{array}$ & $\begin{array}{l}.0252 \\
.0271 \\
.0290 \\
.0310 \\
.0324\end{array}$ \\
\hline $\begin{array}{l}11 \\
12 \\
14 \\
16 \\
18\end{array}$ & $\begin{array}{l}.00140 \\
.00148 \\
.00164 \\
.00180 \\
.00196\end{array}$ & $\begin{array}{l}.0224 \\
.0237 \\
.0262 \\
.0288 \\
.0314\end{array}$ & $\begin{array}{l}.150 \\
.190 \\
.210 \\
.230 \\
.250\end{array}$ & $\begin{array}{l}.0458 \\
.0484 \\
.0534 \\
.0586 \\
.0638\end{array}$ & $\begin{array}{l}.0343 \\
.0362 \\
.0400 \\
.0439 \\
.0478\end{array}$ \\
\hline $\begin{array}{l}20 \\
22 \\
24 \\
26 \\
28\end{array}$ & $\begin{array}{l}.00212 \\
.00226 \\
.00242 \\
.00258 \\
.00274 \\
.00288\end{array}$ & $\begin{array}{l}.0339 \\
.0362 \\
.0387 \\
.0413 \\
.0438 \\
.0461\end{array}$ & $\begin{array}{l}.270 \\
.290 \\
.310 \\
.330 \\
.350 \\
.370\end{array}$ & $\begin{array}{r}.0688 \\
.0734 \\
.0784 \\
.0836 \\
.0886 \\
.0932\end{array}$ & $\begin{array}{l}.0516 \\
.0550 \\
.0588 \\
.0626 \\
.0664 \\
.0698\end{array}$ \\
\hline
\end{tabular}

1 Equivalent angular $\nabla$ ariation expressed as a decimal part of one turn.

Maximum amount it is possible for new working plug and ring gages which come within the specified tolerances to vary from being flush at the small end or at the gaging notch when screwed together tight by

hand ( 2 times column $3+0.0010$ inch).
3 Maximum amount it is possible for new working plug or ring gages which come within specified tolerances to vary from being flush at the small end or at the gaging notch when screwed on reference gage tight bp band. $\left\{\frac{\text { Column 5, Table } 77+\text { column } 5 \text {, Table } 78 .}{2}\right\}$

TABLE 79.-Corrections in diameter for errors in half angle, national (American Briggs') taper pipe thread gages

\begin{tabular}{|c|c|c|c|c|c|}
\hline \multirow{2}{*}{ Error in half angle of thread in minutes, $a^{\prime}$} & \multicolumn{5}{|c|}{ Correction in diameter, $E^{\prime \prime}$} \\
\hline & $\begin{array}{l}8 \text { threads } \\
\text { per inch }\end{array}$ & $\begin{array}{c}11 \frac{1}{2} \text { threads } \\
\text { per inch }\end{array}$ & $\begin{array}{l}14 \text { threads } \\
\text { per inch }\end{array}$ & $\begin{array}{l}18 \text { threads } \\
\text { per inch }\end{array}$ & $\begin{array}{l}27 \text { threads } \\
\text { pcr inch }\end{array}$ \\
\hline 1 & 2 & 3 & 4 & 5 & 6 \\
\hline $\begin{array}{l}4 \\
4\end{array}$ & $\begin{array}{l}\text { Inch } \\
0.00006 \\
.00011 \\
.00017 \\
.00022 \\
.00028\end{array}$ & $\begin{array}{l}\text { Inch } \\
0.00004 \\
.00008 \\
.00012 \\
.00016 \\
.00019\end{array}$ & $\begin{array}{l}\text { Inch } \\
0.00003 \\
.00006 \\
.00010 \\
.00013 \\
.00016\end{array}$ & $\begin{array}{l}\text { Inch } \\
0.00002 \\
.00005 \\
.00007 \\
.00010 \\
.00012\end{array}$ & $\begin{array}{l}\text { Inch } \\
0.00002 \\
.00003 \\
.00005 \\
.00007 \\
.00008\end{array}$ \\
\hline $\begin{array}{l}6 \\
7 \\
8 \\
8\end{array}$ & $\begin{array}{l}.00034 \\
.00039 \\
.00045 \\
.00050 \\
.00056\end{array}$ & $\begin{array}{l}.00023 \\
.00027 \\
.00031 \\
.00035 \\
.00039\end{array}$ & $\begin{array}{l}.00019 \\
.00022 \\
.00026 \\
.00029 \\
.00032\end{array}$ & $\begin{array}{l}.00015 \\
.00017 \\
.00020 \\
.00022 \\
.00025\end{array}$ & $\begin{array}{l}.00010 \\
.00012 \\
.00013 \\
.00015 \\
.00017\end{array}$ \\
\hline
\end{tabular}


TABLE 79.-Corrections in diameter for errors in half angle, national (American Briggs') taper pipe thread gages-Continued

\begin{tabular}{|c|c|c|c|c|c|}
\hline \multirow{2}{*}{ Error in half angle of thread in minutes, $a^{\prime}$} & \multicolumn{5}{|c|}{ Correction in diameter, $E^{\prime \prime}$} \\
\hline & $\begin{array}{l}8 \text { threads } \\
\text { per inch }\end{array}$ & $\begin{array}{l}111 / 2 \text { threads } \\
\text { per inch }\end{array}$ & $\begin{array}{l}14 \text { threads } \\
\text { per inch }\end{array}$ & $\begin{array}{l}18 \text { threads } \\
\text { per inch }\end{array}$ & $\begin{array}{l}27 \text { threads } \\
\text { per inch }\end{array}$ \\
\hline 1 & $?$ & 3 & 4 & 5 & 6 \\
\hline $11{ }_{11}$ & $\begin{array}{l}\text { Inch } \\
0.00062 \\
.00067 \\
.00073 \\
.00078 \\
.00081\end{array}$ & $\begin{array}{l}\text { Inch } \\
0.00043 \\
.00047 \\
.00051 \\
.00054 \\
.00058\end{array}$ & $\begin{array}{l}\text { Inch } \\
0.00035 \\
.00038 \\
.00042 \\
.00045 \\
.00048\end{array}$ & $\begin{array}{l}\text { Inch } \\
0.00027 \\
.00030 \\
.00032 \\
.00035 \\
.00037\end{array}$ & $\begin{array}{r}\text { Inch } \\
0.00018 \\
.00020 \\
.00022 \\
.00023 \\
.00025\end{array}$ \\
\hline $\begin{array}{l}16 \\
17 \\
18 \\
19\end{array}$ & $\begin{array}{l}.00089 \\
.00095 \\
.00101 \\
.00106 \\
.00112\end{array}$ & $\begin{array}{l}.00062 \\
.00066 \\
.00070 \\
.00074 \\
.00078\end{array}$ & $\begin{array}{l}.00051 \\
.00054 \\
.00058 \\
.00061 \\
.00064\end{array}$ & $\begin{array}{l}.00040 \\
.00042 \\
.00045 \\
.00047 \\
.00050\end{array}$ & $\begin{array}{l}.00027 \\
.00028 \\
.00030 \\
.00031 \\
.00033\end{array}$ \\
\hline $22^{21}$ & $\begin{array}{l}.00117 \\
.00123 \\
.00129 \\
.00134 \\
.00140\end{array}$ & $\begin{array}{r}.00082 \\
.00086 \\
.00089 \\
.00093 \\
.00097\end{array}$ & $\begin{array}{l}.00067 \\
.00070 \\
.00074 \\
.00077 \\
.00080\end{array}$ & $\begin{array}{l}.00052 \\
.00055 \\
.00057 \\
.00060 \\
.00062\end{array}$ & $\begin{array}{l}.00035 \\
.00036 \\
.00038 \\
.00040 \\
.00041\end{array}$ \\
\hline $\begin{array}{l}26 \\
27 \\
28\end{array}$ & $\begin{array}{l}.00145 \\
.00151 \\
.00157 \\
.00162 \\
.00168\end{array}$ & $\begin{array}{l}.00101 \\
.00105 \\
.00109 \\
.00113 \\
.00117\end{array}$ & $\begin{array}{l}.00083 \\
.00086 \\
.00089 \\
.00093 \\
.00096\end{array}$ & $\begin{array}{l}.00065 \\
.00067 \\
.00070 \\
.00072 \\
.00075\end{array}$ & $\begin{array}{l}.00043 \\
.00045 \\
.00046 \\
.00048 \\
.00050\end{array}$ \\
\hline 65 & $\begin{array}{l}.00252 \\
.00336\end{array}$ & $\begin{array}{l}.00175 \\
.00233\end{array}$ & $\begin{array}{l}.00144 \\
.00192\end{array}$ & $\begin{array}{l}.00112 \\
.00149\end{array}$ & .00075 \\
\hline
\end{tabular}

$a^{\prime}=$ error in half included angle of thread. $E^{\prime \prime}=$ correction in diameter. $E^{\prime \prime}=\frac{1.53812}{n} \times \tan a^{\prime}$.

TABLE 80.-Corrections in diameter for errors in lead

\begin{tabular}{|c|c|c|c|c|c|c|c|c|c|c|}
\hline \multirow{2}{*}{$\begin{array}{l}\text { Error in } \\
\text { lead in } \\
\text { inches, } p^{\prime}\end{array}$} & \multicolumn{10}{|c|}{ Correction in äiameter, $E^{\prime}$} \\
\hline & 0.00000 & 0.00001 & 0.00002 & 0.00003 & 0.00001 & 0.00005 & 0.00006 & 0.00007 & 0.00008 & 0.00009 \\
\hline 1 & 2 & 3 & 4 & 5 & 6 & $\gamma$ & 8 & 9 & 10 & 11 \\
\hline $\begin{array}{l}0.00000 \\
.00010 \\
.00020 \\
.00030 \\
.00010 \\
.00050\end{array}$ & $\begin{array}{c}\text { Inch } \\
0.00000 \\
.00017 \\
.00035 \\
.00052 \\
.00069 \\
.00087\end{array}$ & $\begin{array}{c}\text { Inch } \\
0.00002 \\
.00019 \\
.00036 \\
.00054 \\
.00071 \\
.00088\end{array}$ & $\begin{array}{c}\text { Inch } \\
0.00003 \\
.00021 \\
.00038 \\
.00955 \\
.00073 \\
.00090\end{array}$ & $\begin{array}{c}\text { Inch } \\
0.00005 \\
.00023 \\
.00040 \\
.00057 \\
.00074 \\
.00092\end{array}$ & $\begin{array}{c}\text { Inch } \\
0.00007 \\
.00024 \\
.00042 \\
.00059 \\
.00076 \\
.00094\end{array}$ & $\begin{array}{c}\text { Inch } \\
0.00009 \\
.00026 \\
.00043 \\
.00061 \\
.00078 \\
.00095\end{array}$ & $\begin{array}{c}\text { Inch } \\
0.00010 \\
.00028 \\
.00015 \\
.00062 \\
.00080 \\
.00097\end{array}$ & $\begin{array}{c}\text { Inch } \\
0.00012 \\
.00029 \\
.00047 \\
.00064 \\
.00081 \\
.00099\end{array}$ & $\begin{array}{c}\text { Inch } \\
0.00014 \\
.00031 \\
.00048 \\
.00066 \\
.00083 \\
.00100\end{array}$ & $\begin{array}{l}\text { Inch } \\
0.00016 \\
.00033 \\
.00050 \\
.00068 \\
.00085 \\
.00102\end{array}$ \\
\hline $\begin{array}{l}.00060 \\
.00070 \\
.00080 \\
.00090 \\
.00100\end{array}$ & $\begin{array}{l}.00104 \\
.00121 \\
.00139 \\
.00156 \\
.00173\end{array}$ & $\begin{array}{l}.00106 \\
.00123 \\
.00140 \\
.00158 \\
.00175\end{array}$ & $\begin{array}{l}.00107 \\
.00125 \\
.00142 \\
.00159 \\
.00177\end{array}$ & $\begin{array}{l}.00109 \\
.00126 \\
.00141 \\
.00161 \\
.00178\end{array}$ & $\begin{array}{l}.00111 \\
.00128 \\
.00145 \\
.00163 \\
.00180\end{array}$ & $\begin{array}{l}.00113 \\
.00130 \\
.00147 \\
.00165 \\
.00182\end{array}$ & $\begin{array}{l}.00114 \\
.00132 \\
.00149 \\
.00166 \\
.00181\end{array}$ & $\begin{array}{l}.00116 \\
.00133 \\
.00151 \\
.00168 \\
.00185\end{array}$ & $\begin{array}{r}.00118 \\
.00135 \\
.00152 \\
.00170 \\
.00187\end{array}$ & $\begin{array}{l}.00120 \\
.00137 \\
.00154 \\
.00171 \\
.00189\end{array}$ \\
\hline $\begin{array}{l}.00110 \\
.00120 \\
.00130 \\
.00140 \\
.00150\end{array}$ & $\begin{array}{l}.00191 \\
.00208 \\
.00225 \\
.00242 \\
.00260\end{array}$ & $\begin{array}{l}.00192 \\
.00210 \\
.00227 \\
.00244 \\
.00262\end{array}$ & $\begin{array}{l}.00194 \\
.00211 \\
.00229 \\
.00246 \\
.00263\end{array}$ & $\begin{array}{l}.00196 \\
.00213 \\
.00230 \\
.00248 \\
.00265\end{array}$ & $\begin{array}{l}.00197 \\
.00215 \\
.00232 \\
.00249 \\
.00267\end{array}$ & $\begin{array}{l}.00199 \\
.00217 \\
.00234 \\
.00251 \\
.00268\end{array}$ & $\begin{array}{l}.00201 \\
.00218 \\
.00236 \\
.00253 \\
.00270\end{array}$ & $\begin{array}{r}.00203 \\
.00220 \\
.00237 \\
.00255 \\
.00272\end{array}$ & $\begin{array}{l}.00204 \\
.00222 \\
.00239 \\
.00256 \\
.00274\end{array}$ & $\begin{array}{l}.00206 \\
.00223 \\
.00241 \\
.00258 \\
.00275\end{array}$ \\
\hline $\begin{array}{l}.00160 \ldots \\
.00170_{-} \\
.00180 \\
.00190_{-}= \\
.00200_{-}\end{array}$ & $\begin{array}{l}.00277 \\
.00291 \\
.00312 \\
.00329 \\
.00346\end{array}$ & $\begin{array}{l}.00279 \\
.00296 \\
.00313 \\
.00331 \\
.00348\end{array}$ & $\begin{array}{l}.00281 \\
.00298 \\
.00315 \\
.00333 \\
.00350\end{array}$ & $\begin{array}{l}.00282 \\
.00300 \\
.00317 \\
.00334 \\
.00352\end{array}$ & $\begin{array}{l}.00284 \\
.00301 \\
.00319 \\
.00336 \\
.00353\end{array}$ & $\begin{array}{l}.00286 \\
.00303 \\
.00320 \\
.00338 \\
.00355\end{array}$ & $\begin{array}{r}.00288 \\
.00305 \\
.00322 \\
.00339 \\
.00357\end{array}$ & $\begin{array}{r}.00289 \\
.00307 \\
.00321 \\
.00341 \\
.00359\end{array}$ & $\begin{array}{l}.00291 \\
.00308 \\
.00326 \\
.00343 \\
.00360\end{array}$ & $\begin{array}{r}.00293 \\
.00310 \\
.00327 \\
.00345 \\
.00362\end{array}$ \\
\hline
\end{tabular}

$p^{\prime}=$ error in lead.

$E^{\prime}=$ correction in diameter.

$E^{\prime}=1.732 p^{\prime}$ 


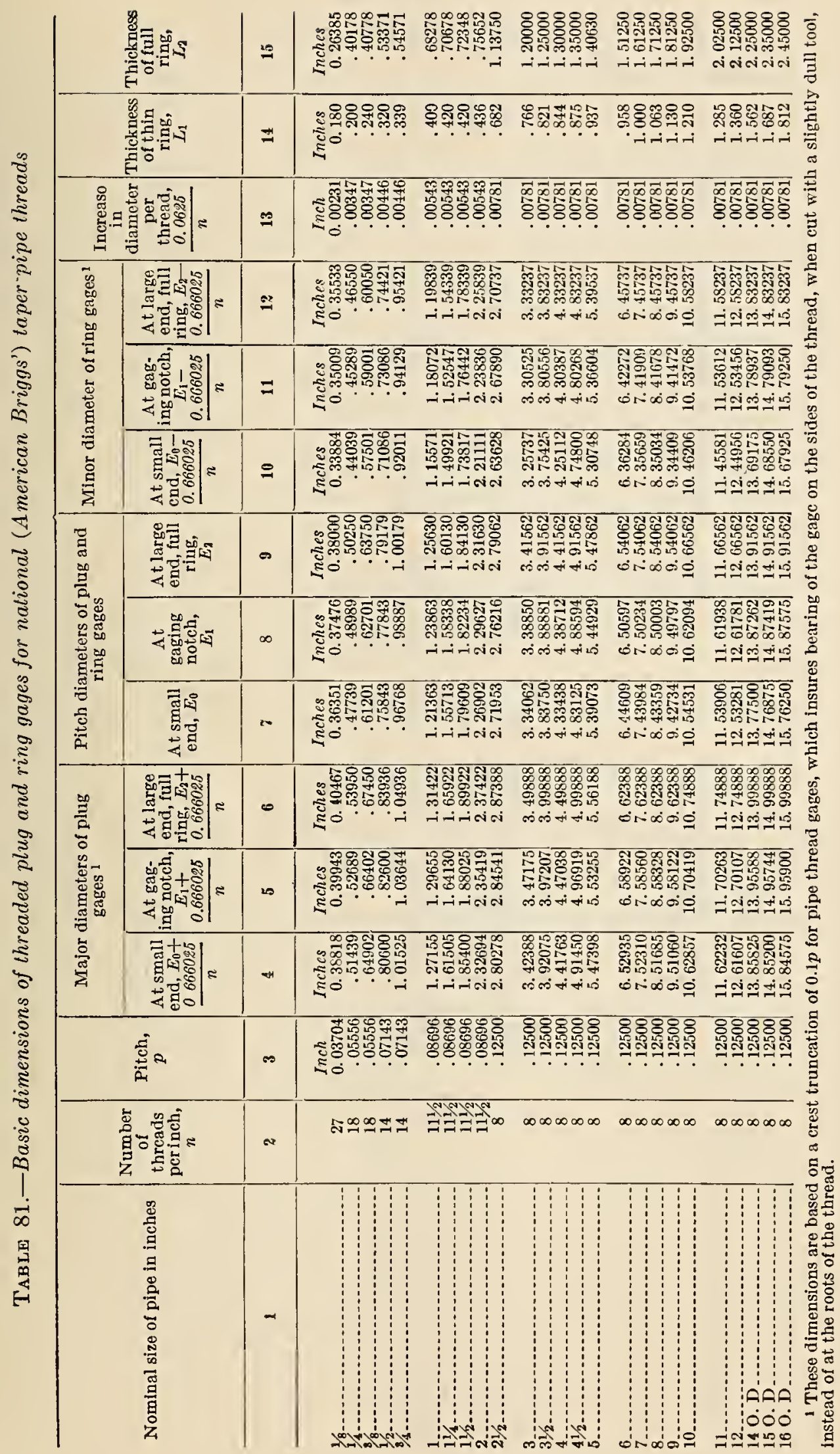




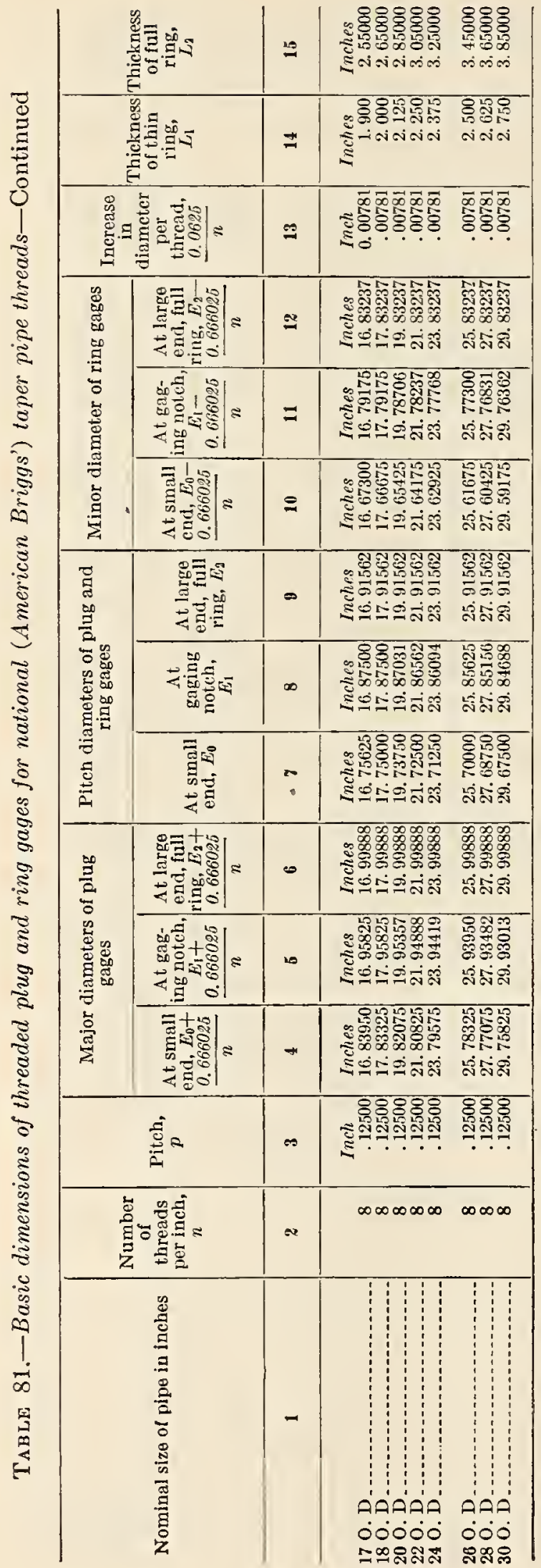




\section{SECTION VII. WOOD SCREWS}

This specification summarizes the results of the standardization of wood screws by the manufacturers in cooperation with the Bureau of Standards and the technical committee on builders' hardware of the Federal Specifications Board. It has been officially adopted by the Federal Specifications Board for the use of all departments and independent establishments of the Government in the purchase of wood screws, and published as Circular No. 140 of the Bureau of Standards.

The former difference of 0.013165 inch in diameter, used as a basis for the arithmetical progression of the numbering system, has been discarded in favor of a difference of 0.013 inch (even) as established by the commission for machine screws. This provides interchangeability for the numbered sizes of machine screws and wood screws in connection with articles that may be fastened either to metal or wood.

Flat, round, and oval head types are covered in the specification.

The numbered sizes of wood screws run consecutively from No. 0 (0.060 inch, or $1.5 \mathrm{~mm})$ to No. 24 (0.372 inch, or $9.4 \mathrm{~mm})$, omitting Nos. $13,15,17,19,21,22$, and 23.

The number of sizes of brass and steel screws manufactured as standard have been reduced from 555 to 291, a reduction of 47 per cent.

\section{GENERAL SPECIFICATIONS}

(a) Material and Workmanship.-Screws shall be made of steel or brass, as specified, and shall be free from any defects which would affect their serviceability.

(b) Pornts. - Standard screws shall be furnished with gimlet points. Cone and diamond pointed screws are special.

(c) Trpes.-Screws shall be furnished in flat, round or oval heads as ordered. (See fig. 37.)

(d) Measurements of Lengths.-The length of all screws shall be measured from the largest diameter of bearing surface of the head to the extreme end of the point measured parallel to the axis of the screw.

(e) Threaded Length.-Screws shall be threaded approximately two-thirds of the nominal length.

(f) Measurement of Diameters.-The diameter shall be measured on the body of the screw under the head.

(g) Tolerance on Diameter.-The maximum variation on diameter permitted is +0.004 and -0.007 inch, or +0.1 and -0.2 mm. 
(h) Tolerance on Number of Threads Per Inch.-The maximum variation in the number of threads per inch permitted is plus or minus 10 per cent.

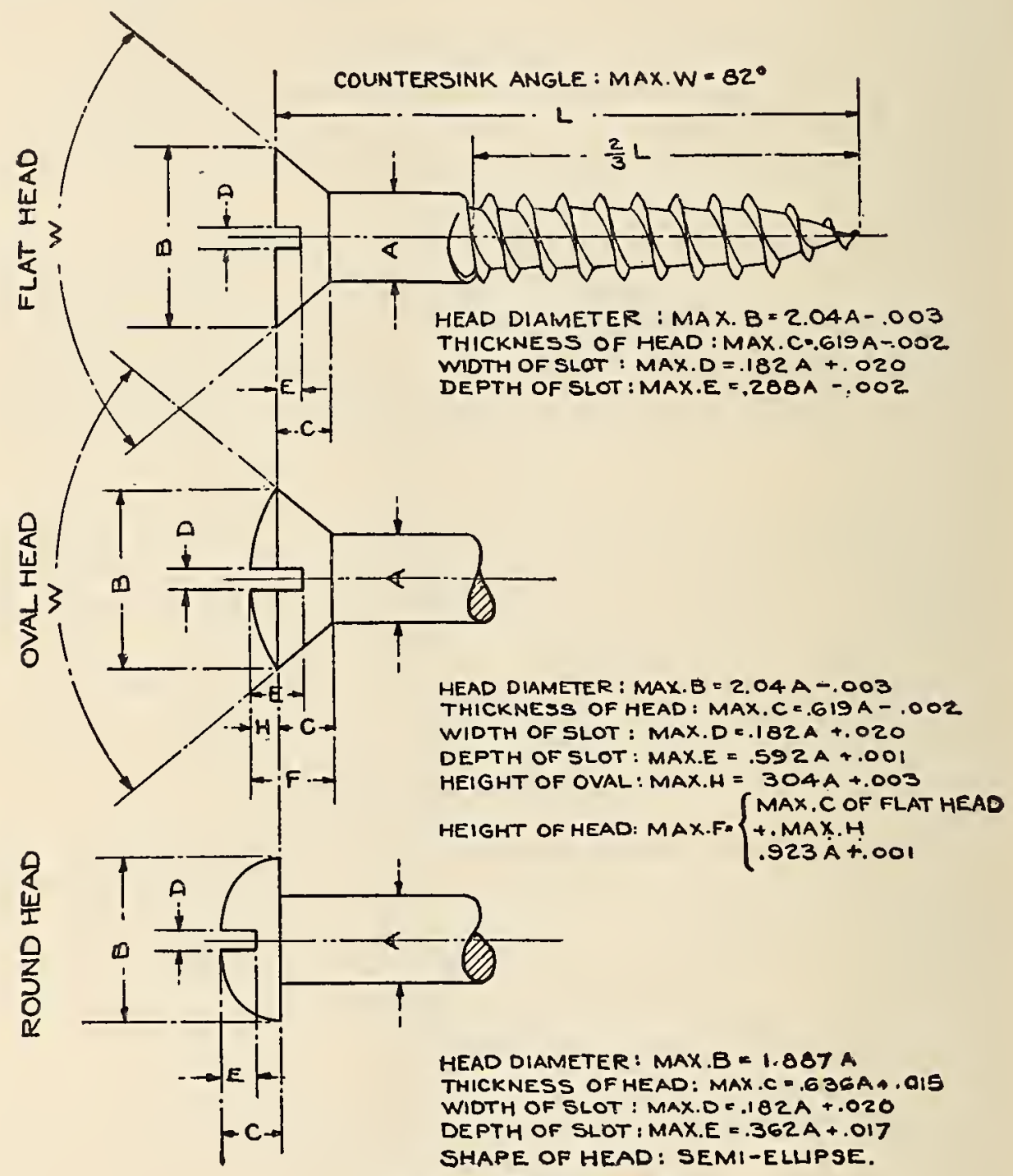

FIG. 37.-Wood screws

(i) INCLUDed ANgLe.-The included angle of the head on flat and oval hea,d screws shall be $82^{\circ}$. 


\section{THREAD SERIES}

The numbered screw sizes, basic and limiting diameters, and threads per inch given in Table 82 are standard.

TABLE 82.-National wood screw standard, size numbers, diameters, and pitches

\begin{tabular}{|c|c|c|c|c|c|c|c|}
\hline \multirow{2}{*}{ Number of screw } & \multirow{2}{*}{$\begin{array}{l}\text { Threads } \\
\text { per inch }\end{array}$} & \multicolumn{6}{|c|}{ Diameter } \\
\hline & & Basic & Maximum & Minimum & Basic & Maximum & Minimum \\
\hline 1 & 2 & 3 & 4 & 5 & 6 & z & 8 \\
\hline $\begin{array}{l}0 \ldots . . \\
1 \\
2 \\
3 \\
4 \ldots . .\end{array}$ & $\begin{array}{l}32 \\
28 \\
26 \\
24 \\
22\end{array}$ & $\begin{array}{r}\text { Inch } \\
0.060 \\
.073 \\
.086 \\
.099 \\
.112\end{array}$ & $\begin{array}{c}\text { Inch } \\
0.064 \\
.077 \\
.090 \\
.103 \\
.116\end{array}$ & $\begin{array}{l}\text { Inch } \\
0.053 \\
.066 \\
.079 \\
.092 \\
.105\end{array}$ & $\begin{array}{r}m m \\
1.5 \\
1.9 \\
2.2 \\
2.5 \\
2.8\end{array}$ & $\begin{array}{r}m m \\
1.6 \\
2.0 \\
2.3 \\
2.6 \\
2.9\end{array}$ & $\begin{array}{r}m m \\
1.3 \\
1.7 \\
2.0 \\
2.3 \\
2.6\end{array}$ \\
\hline $\begin{array}{l}5-10 \\
6 \\
8 \\
8 \\
9\end{array}$ & $\begin{array}{l}20 \\
18 \\
16 \\
15 \\
14\end{array}$ & $\begin{array}{l}.125 \\
.138 \\
.151 \\
.164 \\
.177\end{array}$ & $\begin{array}{l}.129 \\
.142 \\
.155 \\
.168 \\
.181\end{array}$ & $\begin{array}{l}.118 \\
.131 \\
.144 \\
.157 \\
.170\end{array}$ & $\begin{array}{l}\text { 3. } 2 \\
3.5 \\
3.8 \\
4.2 \\
4.5\end{array}$ & $\begin{array}{l}3.3 \\
3.6 \\
3.9 \\
4.3 \\
4.6\end{array}$ & $\begin{array}{l}3.0 \\
3.3 \\
3.6 \\
4.0 \\
4.3\end{array}$ \\
\hline $\begin{array}{l}10 \\
11 \\
12 \\
14 \\
16\end{array}$ & $\begin{array}{r}13 \\
12 \\
11 \\
10 \\
9\end{array}$ & $\begin{array}{l}.190 \\
.203 \\
.216 \\
.242 \\
.268\end{array}$ & $\begin{array}{l}.194 \\
.207 \\
.220 \\
.246 \\
.272\end{array}$ & $\begin{array}{l}.183 \\
.196 \\
.209 \\
.235 \\
.261\end{array}$ & $\begin{array}{l}4.8 \\
5.2 \\
5.5 \\
6.1 \\
6.8\end{array}$ & $\begin{array}{l}4.9 \\
5.3 \\
5.6 \\
6.2 \\
6.9\end{array}$ & $\begin{array}{l}4.6 \\
5.0 \\
5.3 \\
5.9 \\
6.6\end{array}$ \\
\hline $\begin{array}{l}18 \\
20 \\
24\end{array}$ & $\begin{array}{l}8 \\
8 \\
7\end{array}$ & $\begin{array}{r}.294 \\
.320 \\
.372\end{array}$ & $\begin{array}{l}.298 \\
.324 \\
.376\end{array}$ & $\begin{array}{l}.287 \\
.313 \\
.365\end{array}$ & $\begin{array}{l}7.5 \\
8.1 \\
9.4\end{array}$ & $\begin{array}{l}7.6 \\
8.2 \\
9.5\end{array}$ & $\begin{array}{l}7.3 \\
7.9 \\
9.2\end{array}$ \\
\hline
\end{tabular}

\section{TOLERANCES ON LENGTH}

(a) Flat and Oval Head Screws.-The maximum variations permitted in the length of flat and oval head screws are given in Table 83.

(b) Round Head Screws.-The maximum variations permitted in the length of round head screws are given in Table 84 .

TABLE 83.-Tolerances on length of flat and oval head screws ${ }^{1}$

\begin{tabular}{|c|c|c|c|}
\hline Nominal length in inches & $\begin{array}{l}\text { Minus } \\
\text { tolerance }\end{array}$ & Nominal length in inches & $\begin{array}{l}\text { Minus } \\
\text { tolerance }\end{array}$ \\
\hline 1 & 2 & 1 & 2 \\
\hline $\begin{array}{l}1 / 4 \\
8 / 8 \\
1 / 2 \\
5 / 8 \\
8 / 4\end{array}$ & $\begin{array}{l}\text { Inch } \\
0.031 \\
.033 \\
.035 \\
.037 \\
.039\end{array}$ & 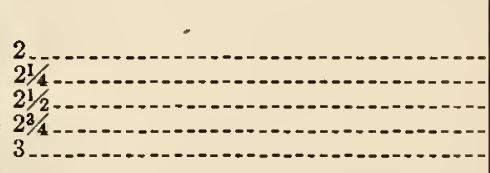 & $\begin{array}{r}\text { Inch } \\
0.060 \\
.064 \\
.068 \\
.072 \\
.076\end{array}$ \\
\hline $1 / 8$ & $\begin{array}{l}.041 \\
.043 \\
.048 \\
.052 \\
.056\end{array}$ & $41 / 2$ & $\begin{array}{l}.084 \\
.092 \\
.101 \\
.109\end{array}$ \\
\hline
\end{tabular}

1 Plus tolerance $=0$.

$16802^{\circ}-25 \dagger-10$ 
TABLE 84.-Tolerances on length of round head screws ${ }^{1}$

\begin{tabular}{|c|c|c|c|c|c|c|c|c|c|}
\hline \multirow{2}{*}{ Nominal length in inches } & \multicolumn{9}{|c|}{ Screw numbers } \\
\hline & 0 & 1 & 2 & 3 & 4 & 5 & 6 & 7 & 8 \\
\hline \multirow{2}{*}{$\begin{array}{l}1 / 46 \\
3 / 8 \\
1 / 2 \\
5 / 8 \\
3 / 4-2\end{array}$} & \multirow{2}{*}{$\begin{array}{l}\text { Inch } \\
0.064 \\
.065\end{array}$} & \multirow{2}{*}{$\begin{array}{l}\text { Inch } \\
0.071 \\
.073 \\
.075 \\
\end{array}$} & \multirow{2}{*}{$\begin{array}{l}\text { Inch } \\
0.077 \\
.079 \\
.081 \\
.083 \\
.085\end{array}$} & \multirow{2}{*}{$\begin{array}{c}\text { Inch } \\
0.084 \\
.086 \\
.088 \\
.090 \\
.092\end{array}$} & \multirow{2}{*}{$\begin{array}{l}\text { Inch } \\
0.090 \\
.092 \\
.094 \\
.096 \\
.098\end{array}$} & Inch & Inch & Inch & Inch \\
\hline & & & & & & $\begin{array}{r}0.099 \\
.101 \\
.103 \\
.105\end{array}$ & $\begin{array}{r}0.105 \\
.107 \\
.109 \\
.111\end{array}$ & $\begin{array}{r}0.112 \\
.114 \\
.116 \\
.118\end{array}$ & $\begin{array}{r}0.118 \\
.120 \\
.122 \\
.124\end{array}$ \\
\hline & 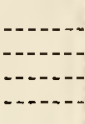 & & & $\begin{array}{l}.094 \\
.096\end{array}$ & $\begin{array}{l}.100 \\
.102 \\
.106 \\
.110\end{array}$ & $\begin{array}{l}.107 \\
.109 \\
.113 \\
.117\end{array}$ & $\begin{array}{l}.113 \\
.115 \\
.119 \\
.123\end{array}$ & $\begin{array}{l}.120 \\
.122 \\
.126 \\
.130\end{array}$ & $\begin{array}{l}.126 \\
.128 \\
.132 \\
.136\end{array}$ \\
\hline & & & & & & & $\begin{array}{l}.127 \\
.131 \\
.135 \\
.139\end{array}$ & $\begin{array}{l}.134 \\
.138 \\
.142 \\
.146\end{array}$ & $\begin{array}{l}.140 \\
.144 \\
.148 \\
.152\end{array}$ \\
\hline \multirow{2}{*}{ Nominal length in inches } & \multicolumn{9}{|c|}{ Screw numbers } \\
\hline & 9 & 10 & 11 & 12 & 14 & 16 & 18 & 20 & 24 \\
\hline \multirow{2}{*}{$\begin{array}{l}1 / 8 \\
8 / 8 \\
3 / 4 \\
3 / 8 \\
1\end{array}$} & \multirow{2}{*}{$\begin{array}{l}\text { Inch } \\
0.127 \\
.129 \\
.131 \\
.133 \\
.135\end{array}$} & \multirow{2}{*}{$\begin{array}{l}\text { Inch } \\
0.133 \\
.135 \\
.137 \\
.139 \\
.141\end{array}$} & Inch & \multirow{2}{*}{$\begin{aligned} & \\
& 0.148 \\
& .150 \\
& .152 \\
& .154\end{aligned}$} & Inch & Inch & Inch & Inch & Inch \\
\hline & & & $\begin{array}{l}0.142 \\
0144 \\
.146 \\
.148\end{array}$ & & $\begin{array}{r}0.163 \\
.165 \\
.167\end{array}$ & $0.180^{\circ}$ & & & -... \\
\hline $\begin{array}{l}1 / 4- \\
11 / 2- \\
13 / 4- \\
2-\end{array}$ & $\begin{array}{l}.139 \\
.143 \\
.147 \\
.151\end{array}$ & $\begin{array}{l}.145 \\
.149 \\
.153 \\
.157\end{array}$ & $\begin{array}{l}.152 \\
.156 \\
.160 \\
.164\end{array}$ & $\begin{array}{l}.158 \\
.162 \\
.166 \\
.170\end{array}$ & $\begin{array}{r}.171 \\
.175 \\
.179 \\
.183\end{array}$ & $\begin{array}{l}.184 \\
.188 \\
.192 \\
.196\end{array}$ & $\begin{array}{r}0.198 \\
.202 \\
.206 \\
.210\end{array}$ & $\begin{array}{r}0.215 \\
.219 \\
.223\end{array}$ & - \\
\hline $21 / 40$ & $\begin{array}{l}.155 \\
.159 \\
.163 \\
.167\end{array}$ & $\begin{array}{l}.161 \\
.165 \\
.169 \\
.173\end{array}$ & $\begin{array}{l}.168 \\
.172 \\
.176 \\
.180\end{array}$ & $\begin{array}{l}.174 \\
.178 \\
.182 \\
.186\end{array}$ & $\begin{array}{l}.187 \\
.191 \\
.195 \\
.199\end{array}$ & $\begin{array}{l}.200 \\
.204 \\
.208 \\
.212\end{array}$ & $\begin{array}{l}.214 \\
.218 \\
.222 \\
.226\end{array}$ & $\begin{array}{l}.227 \\
.231 \\
.235 \\
.239\end{array}$ & 0.265 \\
\hline $\begin{array}{l}31 / 2 \\
4 i \overline{2} \\
41 / 2 \\
5-.\end{array}$ & & .181 & .188 & $\begin{array}{l}.194 \\
.202\end{array}$ & $\begin{array}{l}.207 \\
.215 \\
.223 \\
.231\end{array}$ & $\begin{array}{l}.220 \\
.228 \\
.226 \\
.244\end{array}$ & $\begin{array}{l}.234 \\
.242 \\
.250 \\
.258\end{array}$ & $\begin{array}{l}.247 \\
.255 \\
.263 \\
.271\end{array}$ & $\begin{array}{l}.273 \\
.281 \\
.289 \\
.297\end{array}$ \\
\hline
\end{tabular}

1 Plus tolerance $=0$. Minus tolerances as given in body of table.

\section{STANDARD SIZES OF WOOD SCREWS}

(a) Steel Sonews.-The standard sizes of steel screws are given in Table 85.

(b) Brass Screws.-The standard sizes of brass screws are given in Table 86. 
TABLE 85.-Standard sizes of steel screws

\begin{tabular}{|c|c|c|c|c|c|c|c|c|c|c|c|}
\hline \multicolumn{3}{|c|}{ Lengths } & $\begin{array}{l}\text { No. } 0 \text {, } \\
\text { diam- } \\
\text { eter, } \\
0.060 \\
\text { inch, } \\
1.5 \mathrm{~mm}\end{array}$ & $\begin{array}{l}\text { No. 1, } \\
\text { diam- } \\
\text { eter, } \\
0.073 \\
\text { inch, } \\
1.9 \mathrm{~mm}\end{array}$ & $\begin{array}{l}\text { No. 2, } \\
\text { diam- } \\
\text { eter, } \\
0.086 \\
\text { inch, } \\
2.2 \mathrm{~mm}\end{array}$ & $\begin{array}{l}\text { No. } 3 \text {, } \\
\text { diam- } \\
\text { eter, } \\
0.099 \\
\text { i neh, } \\
2.5 \mathrm{~mm}\end{array}$ & $\begin{array}{l}\text { No. } 4, \\
\text { diam- } \\
\text { eter, } \\
0.112 \\
\text { inch, } \\
2.8 \mathrm{~mm}\end{array}$ & $\begin{array}{l}\text { No. 5, } \\
\text { diam- } \\
\text { eter, } \\
0.125 \\
\text { inch, } \\
3.2 \mathrm{~mm}\end{array}$ & $\begin{array}{l}\text { No. 6, } \\
\text { diam- } \\
\text { eter, } \\
0.138 \\
\text { i nch, } \\
3.5 \mathrm{~mm}\end{array}$ & $\begin{array}{l}\text { No. } 7, \\
\text { diam- } \\
\text { eter, } \\
0.151 \\
\text { inch, } \\
3.8 \mathrm{~mm}\end{array}$ & $\left\{\begin{array}{l}\text { No. } 8 \\
\text { diam- } \\
\text { eter, } \\
0.164 \\
\text { inch, } \\
4.2 \mathrm{~mm}\end{array}\right.$ \\
\hline \multirow[t]{4}{*}{ Inches } & & $m m$ & & & & & & & & & \\
\hline & $\begin{array}{l}3 / 8 \\
1 / 2 \\
5 / 8 \\
3 / 4\end{array}$ & $\begin{array}{r}9.5 \\
12.7 \\
15.9 \\
19.1\end{array}$ & $x$ & $\underset{x}{x}$ & $\begin{array}{l}x \\
x \\
x \\
x\end{array}$ & $\begin{array}{l}\hat{x} \\
x \\
x \\
x\end{array}$ & $\begin{array}{l}x \\
x \\
x \\
x\end{array}$ & $\begin{array}{l}x \\
x \\
x\end{array}$ & $\begin{array}{l}x \\
x \\
x\end{array}$ & $\begin{array}{l}x \\
x \\
x\end{array}$ & $\begin{array}{l}x \\
x \\
x\end{array}$ \\
\hline & $\begin{array}{l}7 / 8 \\
11 / 4 \\
11 / 2 \\
13 / 4\end{array}$ & $\begin{array}{l}22.2 \\
25.4 \\
31.8 \\
38.1 \\
44.5\end{array}$ & & & & $\stackrel{x}{x}$ & $\begin{array}{l}x \\
x \\
x \\
x\end{array}$ & $\begin{array}{l}\stackrel{x}{x} \\
\dot{x} \\
x\end{array}$ & $\begin{array}{l}x \\
x \\
x \\
x \\
x\end{array}$ & $\begin{array}{l}x \\
\stackrel{x}{x} \\
\dot{x} \\
\dot{x} \\
x\end{array}$ & $\begin{array}{l}x \\
\dot{x} \\
\dot{x} \\
x \\
x\end{array}$ \\
\hline & $\begin{array}{l}2 \\
21 / 4 \\
21 / 2 \\
23 / 4 \\
3\end{array}$ & $\begin{array}{l}50.8 \\
57.2 \\
63.5 \\
69.9 \\
76.2\end{array}$ & & & & & & & $\begin{array}{l}x \\
x \\
x\end{array}$ & $\begin{array}{l}x \\
x \\
x\end{array}$. & $\begin{array}{l}\stackrel{x}{x} \\
\stackrel{x}{x}\end{array}$ \\
\hline \multicolumn{3}{|c|}{ Lengths } & $\begin{array}{l}\text { No. } 9 \text {, } \\
\text { diam- } \\
\text { eter, } \\
0.177 \\
\text { inch, } \\
4.5 \mathrm{~mm}\end{array}$ & $\begin{array}{l}\text { No. 10, } \\
\text { diam- } \\
\text { eter, } \\
0.190 \\
\text { i nch, } \\
4.8 \mathrm{~mm}\end{array}$ & $\begin{array}{l}\text { No.11, } \\
\text { diam- } \\
\text { eter, } \\
0.203 \\
\text { inch, } \\
5.2 \mathrm{~mm}\end{array}$ & $\begin{array}{l}\text { No. 12, } \\
\text { diam- } \\
\text { eter, } \\
0.216 \\
\text { inch, } \\
5.5 \mathrm{~mm}\end{array}$ & $\begin{array}{c}\text { No. 14, } \\
\text { diam- } \\
\text { eter, } \\
0.242 \\
\text { i nch, } \\
6.1 \mathrm{~mm}\end{array}$ & $\begin{array}{l}\text { No. 16, } \\
\text { diam } \\
\text { eter, } \\
0.268, \\
\text { inch; } \\
6.8 \mathrm{~mm}\end{array}$ & $\begin{array}{c}\text { No. 18, } \\
\text { diam- } \\
\text { eter, } \\
0.294 \\
\text { inch, } \\
7.5 \mathrm{~mm}\end{array}$ & $\begin{array}{c}\text { No. 20, } \\
\text { diam- } \\
\text { eter, } \\
0.320 \\
\text { inch, } \\
8.1 \mathrm{~mm}\end{array}$ & $\begin{array}{l}\text { No. 24, } \\
\text { diam- } \\
\text { eter, } \\
0.372 \\
\text { inch, } \\
9.4 \mathrm{~mm}\end{array}$ \\
\hline \multirow[t]{6}{*}{ Inches } & & ${ }^{m m}{ }_{12.7}$ & X & $X$ & & & & & & & \\
\hline & $\begin{array}{l}0 / 8 \\
3 / 4 \\
7 / 8\end{array}$ & $\begin{array}{l}15.9 \\
19.1 \\
22.2\end{array}$ & $\begin{array}{l}x \\
x \\
x\end{array}$ & $\begin{array}{l}x \\
x \\
x\end{array}$ & $\begin{array}{l}\stackrel{x}{x} \\
x \\
x\end{array}$ & $\begin{array}{l}x \\
x \\
x\end{array}$ & $\begin{array}{l}x \\
x\end{array}$ & & & & \\
\hline & $1^{8 /}$ & 25.4 & $x$ & $x$ & $x$ & $\hat{x}$ & $\hat{x}$ & $x$ & 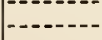 & $\ldots$ & - \\
\hline & $\begin{array}{l}11 / 4 \\
11 / 2 \\
13 / 4 \\
2\end{array}$ & $\begin{array}{l}31.8 \\
38.1 \\
44.5 \\
50.8\end{array}$ & $\begin{array}{l}x \\
x \\
x \\
x\end{array}$ & $\begin{array}{l}x \\
x \\
x \\
x\end{array}$ & $\begin{array}{l}\stackrel{x}{x} \\
\stackrel{x}{x} \\
x\end{array}$ & $\begin{array}{l}x \\
x \\
x \\
x\end{array}$ & $\begin{array}{l}x \\
x \\
x \\
x\end{array}$ & $\begin{array}{l}\dot{x} \\
\dot{x} \\
x \\
x\end{array}$ & $\begin{array}{l}\underset{x}{x} \\
\stackrel{x}{x}\end{array}$ & $\begin{array}{l}x \\
x\end{array}$ & 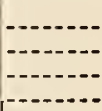 \\
\hline & $\begin{array}{l}21 / 1 \\
21 / 2 \\
23 / 4 \\
3\end{array}$ & $\begin{array}{l}57.2 \\
63.5 \\
69.9 \\
76.2\end{array}$ & $\begin{array}{l}x \\
x \\
x\end{array}$ & $\begin{array}{l}x \\
x \\
x \\
x\end{array}$ & $\begin{array}{l}x \\
x \\
x \\
x \\
x\end{array}$ & $\begin{array}{l}x \\
x \\
x \\
x\end{array}$ & $\begin{array}{l}x \\
x \\
x \\
x\end{array}$ & $\begin{array}{l}\stackrel{x}{x} \\
\dot{x} \\
x\end{array}$ & $\begin{array}{l}\stackrel{x}{x} \\
\dot{x} \\
x\end{array}$ & $\begin{array}{l}\frac{x}{x} \\
\frac{x}{x}\end{array}$ & 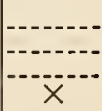 \\
\hline & $\begin{array}{l}31 / 2 \\
4 \\
41 / 2 \\
5\end{array}$ & $\begin{array}{r}88.9 \\
101.6 \\
114.3 \\
127.0\end{array}$ & & $x$ & $\times$ & $\begin{array}{l}x \\
x\end{array}$ & $\begin{array}{l}x \\
x \\
x \\
x\end{array}$ & $\begin{array}{l}x \\
x \\
x \\
x\end{array}$ & $\begin{array}{l}\underset{x}{x} \\
x \\
x\end{array}$ & $\begin{array}{l}\stackrel{x}{x} \\
x \\
x\end{array}$ & $\begin{array}{l}x \\
x \\
x \\
x\end{array}$ \\
\hline
\end{tabular}


TABLE 86.-Standard sizes of brass screws

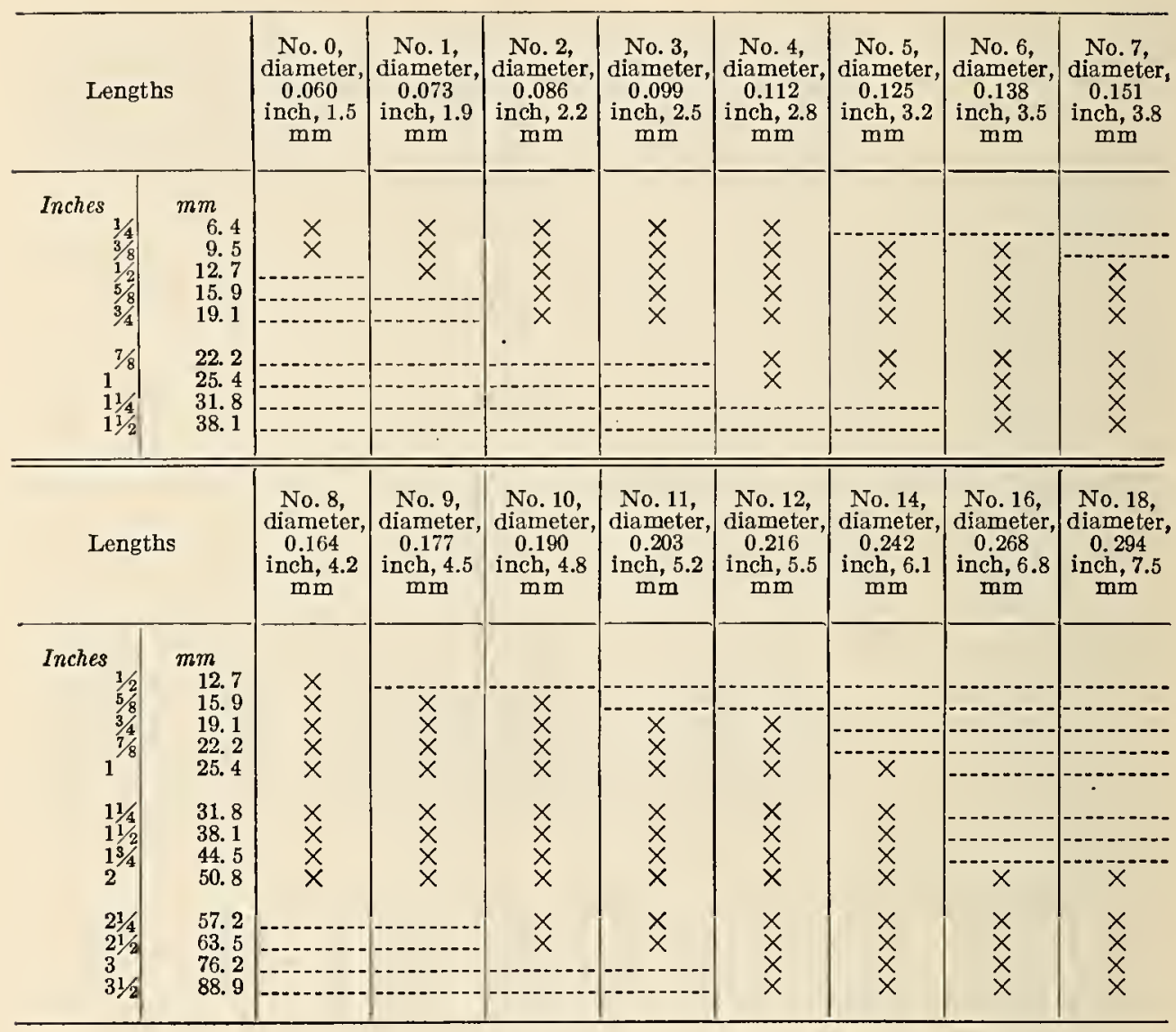




\section{APPENDIX 1. DERIVATION OF TOLERANCES}

\section{PITCH DIAMETER TOLERANCES}

(a) Tolerances for Fastening Screws.-The tolerances for fastening screws specified in Section III were arrived at by combining two factors, known as the net pitch diameter tolerance and the gage tolerance. The theoretical net tolerances for all screws and nuts of a given class of fit bear a definite mathematical relationship to each other, and it was intended that these should in no way be reduced by permissible manufacturing tolerances for master gages; that is, gages within class $\mathrm{X}$ tolerances. Consequently the net tolerances were increased by the equivalent diametrical space required to provide for the class $\mathrm{X}$ tolerances on diameter, lead, and angle, to produce the extreme tolerances specified for the product. In practice, the actual net tolerances will depend upon the method of gaging and upon the accuracy of the gages used.

1. Basis of net tolerances. - The net pitch diameter tolerances for the various classes of fit are based on the following series for a pitch of $\frac{1}{20}$ inch:

Class 1 , loose fit 0. 0045

Class 2, free fit.

Class 3, medium fit__._._._. 0020

Class 4, close fit_._._. 0010

Pitch diameter tolerances for pitches finer than $\frac{1}{2} \frac{1}{0}$ inch are to each other and to the tolerance for $\frac{1}{2}$ inch as the 0.6 th power of their respective pitches.

Pitch diameter tolerances for pitches coarser than $\frac{1}{20}$ inch are to each other and to the tolerance for $\frac{1}{2} \sigma$ inch as the 0.9 th power of their respective pitches.

The exponent 0.6 was chosen for pitches finer than $\frac{1}{20}$ inch because the resulting tolerances, except in two instances, do not vary more than 0.0001 inch from the pitch diameter tolerances specified in the A. S. M. E. Machine Screw Standard.

2. Gage tolerance.-The gage tolerance to be added to the net tolerance to obtain the extreme tolerance, which determines the absolute limits within which all variations of the work must be kept, is determined as follows:

Add together the following:

Pitch diameter tolerance of "go" gage,

Diametrical equivalent of lead tolerance of "go" gage,

Diametrical equivalent of angle tolerance of "go" gage,

Pitch diameter tolerance of "not go" gage.

Then subtract the following from the above sum:

One-half diametrical equivalent of lead tolerance of "not go" gage.

Diametrical equivalent of angle tolerance of "not go" gage.

(b) Tolerances for Screw Threads of Special Diameters, Pitches, and Lengths of Engagement.-As stated in Section IV, the pitch diameter tolerances for special sizes of threads of national form are obtained by adding three values, or increments; one dependent upon the basic major diameter, another upon the length of engagement, and the third upon the pitch. The formulas from which increments are derived are such that if used to derive tolerances for threads having a length of engagement equal to one diameter and falling within the regular series of sizes and classification of fits given in Section III, the resulting values will closely approximate the extreme tolerances specified in Section III. These formulas are summarized in Table 87. 
TABLE 87.-Schedule of tolerance increments for special threads

\begin{tabular}{|c|c|c|c|c|}
\hline Special classification of fit & Corresponding regular classification & $\begin{array}{l}\text { Diameter } \\
\text { increment }\end{array}$ & $\begin{array}{c}\text { Length of } \\
\text { engagement } \\
\text { increment }\end{array}$ & $\begin{array}{l}\text { Pitch in- } \\
\text { crement }\end{array}$ \\
\hline 1 & 2 & 3 & 4 & $\mathbf{5}$ \\
\hline Class $\mathrm{A}$ & Class 1 , loose fit..... & $0.002 \sqrt{D}$ & $0.002 Q$ & $0.020 \sqrt{p}$ \\
\hline Class B. & Class 1 , loose fit (without allowance). & $.002 \sqrt{D}$ & $.002 Q$ & $.020 \sqrt{p}$ \\
\hline Class $\mathrm{C}$. & Class 2, free fit. & $.002 \sqrt{D}$ & $.002 Q$ & $.010 \sqrt{p}$ \\
\hline Class D.. & Class 3 , medium fit. & $.002 \sqrt{D}$ & $.002 Q$ & $.005 \sqrt{p}$ \\
\hline Class $\mathbf{E}$. & Class 4 , close fit.... & $.001 \sqrt{D}$ & $.001 Q$ & $.0025 \sqrt{p}$ \\
\hline
\end{tabular}

\section{RELATION OF LEAD AND ANGLE ERRORS TO PITCH DIAMETER
TOLERANCES}

It has been stated in various sections of the report that the tolerances specified for pitch diameter include all errors of pitch diameter, lead, and angle. Also, there were tabulated the errors in lead and angle, each of which could be compensated for by one-half of the specified pitch diameter tolerances. These equivalents were derived from definite mathematical relations which are given below. A rigorous mathematical analysis upon which these formulas are based is presented in Appendix 3 of Letter Circular No. 23, issued by the Bureau of Standards.

(a) Diameter Equivalent of Lead Error.-The formula expressing the relation between lead error between any two threads within the length of engagement and its diameter equivalent is as follows:

in which

$$
E^{\prime}=\left( \pm p^{\prime}\right) \cot a,
$$

$E^{\prime}=$ pitch diameter increment due to lead error,

$p^{\prime}=$ the maximum lead error between any two of the threads engaged,

$a=$ half-angle of thread.

The quantity $E^{\prime}$ is always added to the measured pitch diameter in the case of external thread, and it is always subtracted in the case of an internal thread, regardless of the sign introduced by the lead error $p^{\prime}$.

For threads of national form, the above formula reduces to:

$$
E^{\prime}=1.7321 p^{\prime}
$$

(b) Diameter Equivalent of Angle Error.- - The general formula expressing the relation between error in the half angle of thread and its diameter equivalent; that is, the amount of the pitch diameter tolerance absorbed by such an error is:

$$
\cot a^{\prime}=\frac{h}{E^{\prime \prime} \sin a \cos a} \pm \cot a
$$

in which

$$
\begin{aligned}
& E^{\prime \prime}=\text { pitch diameter increment due to error in half-angle, } \\
& h=\text { basic thread depth, } \\
& a=\text { basic half-angle of thread, } \\
& a^{\prime}=\text { error in half-angle of thread. }
\end{aligned}
$$

The sign of cot $a$ is plus when the half angle of thread is less than nominal, and minus when the half angle is greater than nominal. By omitting $\pm \cot a$ from the formula an approximate mean value for $a^{\prime}$ or $E^{\prime \prime}$ is obtained which differs 
very little from either extreme value. The commission has, therefore, adopted for general use the formula:

$$
\cot a^{\prime}=\frac{h}{E^{\prime \prime} \sin a \cos a}
$$

For threads of national form this formula reduces to:

$$
\cot a^{\prime}=\frac{3 p}{2 E^{\prime \prime}}
$$

or

$$
E^{\prime \prime}=\frac{3}{2} p \tan a^{\prime}
$$

For the form of thread recommended for pipe-thread gages the formula becomes:

$$
\cot a^{\prime}=\frac{1.53812 p}{E^{\prime \prime}}
$$

or

$$
E^{\prime \prime}=\frac{1.53812}{. n} \tan a^{\prime}
$$

\section{APPENDIX 2. WIRE METHODS OF MEASUREMENT OF PITCH DIAMETER}

Throughout this report emphasis has been placed on pitch-diameter tolerances and limits, as upon these the fit of a screw thread largely depends. The maintenance of these tolerances and limits requires the use of limit thread gages, and these, in turn, depend upon the absolute values or measurements of master gages. The measurement of pitch diameter presents certain difficulties which may result in an uncertainty as to its true value. The adoption of a uniform practice in making such measurement is, therefore, desirable. The so-called "three-wire method" of measuring pitch diameter, as here outlined, has been found to be the most accurate and satisfactory when properly carried out, and is recommended for universal use in the direct measurement of thread plug gages.

\section{SIZE OF WIRES}

In the three-wire method of measuring pitch diameter small hardened steel cylinders or wires of correct size are placed in the thread groove, two on one side of the screw and one on the opposite side, as shown in Figure 38 . The contact face of the micrometer anvil or spindle over the two wires must be sufficiently large in diameter to touch both wires; that is, it must be equal to or greater than the pitch of the thread. It is best to select wires of such a size that they touch the sides of the thread at the mid slope, for the reason that the measurement of pitch diameter is least affected by any error in thread angle which may be present when such size is used. The size of wire which touches exactly at the mid slope of a perfect thread of a given pitch is termed the "bestsize" wire for that pitch. Any size, however, may be used which will permit the wires to rest on the sides of the thread and also project above the top of the thread.

The depth at which a wire of given diameter will rest in a thread groove depends primarily on the pitch and included angle of the thread; and secondarily, on the angle made by the helix, at the point of contact of the wire and the thread, with a plane perpendicular to the axis of the serew. Inasmuch as variation in the helix angle has a very small effect in determining the diameter of the wire which touches at the mid slope of the thread, and as it is desirable to use one size of 
wire to measure all threads of a given pitch and included angle, the best size wire is taken as that size which will touch at the mid slope of a groove cut around a cylinder perpendicular to the axis of the cylinder, and of the same anglc and depth as the thread of the given pitch. This is equivalent to a thread of zero helix angle. The size of wire touching at the mid slope, or "best-size" wire, is given by the formula:

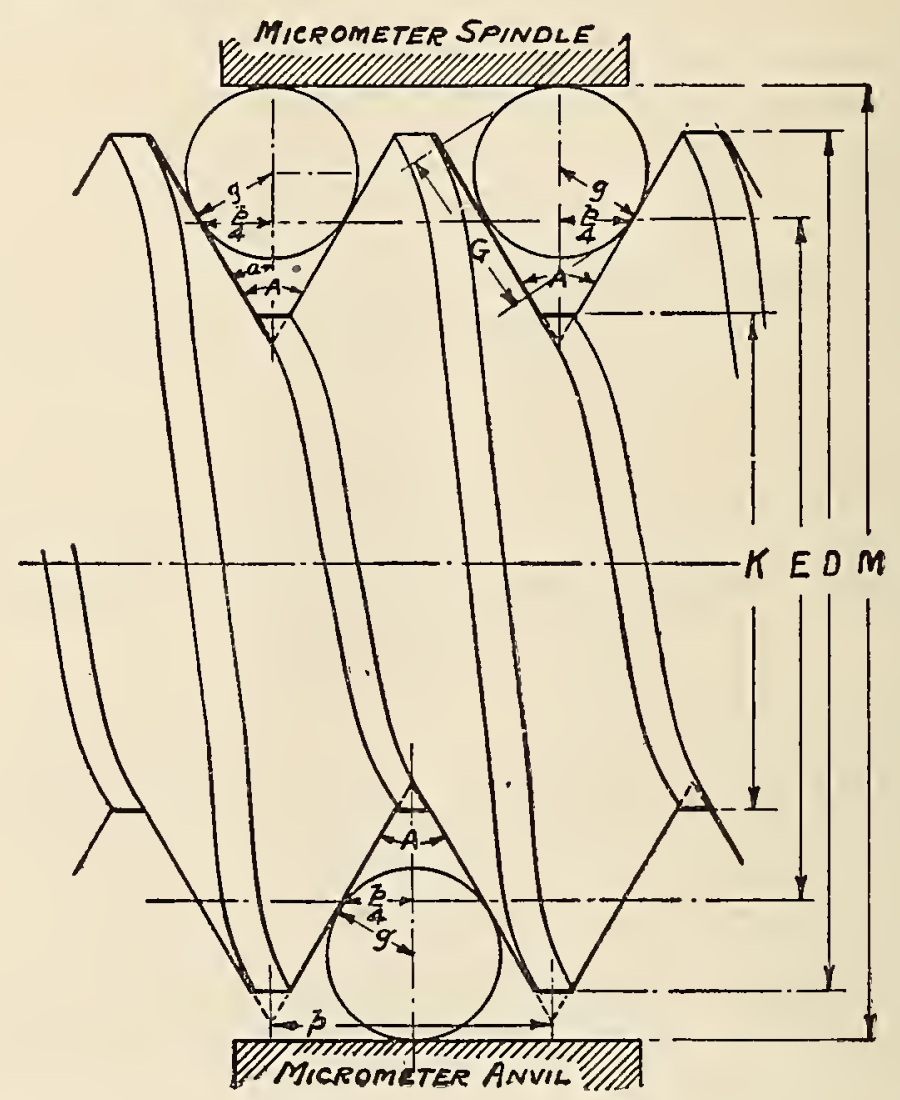

FIa. 38.-Three-wire method of measuring pitch diameter of thread plug gages

in which

$$
G=\frac{p}{2} \sec a
$$

This formula reduces to:

$$
\begin{aligned}
& G=\text { diameter of wire } \\
& p=\text { pitch } \\
& a=1 / 2 \text { included angle of thread. }
\end{aligned}
$$

$$
G=0.57735 \times p, \text { for } 60^{\circ} \text { threads. }
$$

It is frequently desirable, as, for example, when a best-size wire is not available, to measure pitch diameter by means of wires of other than the best size. The minimum size which may be used is limited to that permitting the wire to project above the crest of the thread, and the maximum to that permitting the wire to rest on the sides of the thread just below the crest, and not ride on the crest of the thread. The diameters of the best size, maximum, and minimum wires for "national coarse" and "national fine" threads are given in Tables 88 and 89. 


\section{SPECIFICATION FOR WIRES}

A suitable specification for wircs is as follows:

1. The wires should be cylinders of steel with working surfaces glass hard and accuratcly finished.

2. The working surface should be about 1 inch in length, and the wire should have a suitable handle which is provided at one end with an eye or other suitable means of suspension.

3. One side of the handle, which should be flattened, should be marked with the pitch for which the wire is the best size, and with the diameter of the working part of the wire.

4. The wire should be round within 0.00002 inch and should be straight to 0.00002 inch over any quarter-inch interval.

5. One set of wires should consist of three wires which should have the same diameter within 0.00003 inch, and this common diameter should be within 0.0001 inch of that corresponding to the best size for the pitch for which the wire is to be used.

\section{METHODS OF MEASURING AND USING WIRES}

In order to measure the pitch diameter of a screw-thread gage to an accuracy of 0.0001 inch by means of wires, it is necessary to know the wire diamctcrs to 0.00003 inch. The micrometer to be used for measuring wires should be one which is graduated to ten-thousandths of an inch and upon which hundredthousandths of an inch can be estimated. Such micrometers are available in various forms of precision bench micrometers, and measuring machines. Care should be taken to make sure that the measuring faces of the micrometer are flat and parallel to within 0.00002 inch. The taper of wires can best be determined by measuring between a flat micrometer contact and a cylindrical anvil. Any pits or worn spots on the wires can be detected with the same arrangement. Variations in roundness and straightness are usually determined by rotating the wire between flat contacts one-fourth inch in diameter. However, one form of variation in roundness can only be detected by rotating the wire in a $V$ groove against a flat micrometer contact. The $V$ groove may be the thread space in a hardened and well-finished thread plug gage.

The contact pressure used in making measurements is also an important factor, since the wires, when in use, rest on the sides of the thread, and a given pressure exerted on the top of the thread has a magnified effect in distorting the wire and causing the measurement of the pitch diameter to be slightly less than it should be. In making measurements over the wires inserted in the thread groove, it has been common shop practice to hold the wires down into the thread by means of elastic bands. This has a tendency to prevent the wires from adjusting themselves to the proper position in the thread grooves; thus a false measurement is obtained. In some cases it has also been the practice to support the screw being measured on two wires, which are in turn supported on a horizontal surface, and measuring from this surface to the top of a wire placed in a thread over the gage. If the screw is of large diameter, its weight causes a distortion of the wires and an inaccurate rcading is obtained. For these rcasons these practices should be avoided and subsidiary apparatus for supporting the wires and micrometer should be used.

A contact pressure of 2 pounds is recommended in making wire measurements of screw-thread gages. A skilled inspector naturally uses a light pressure or "feel" in making wire measurements by means of a micrometer caliper. The actual contact pressure used by a group of inspectors was found to vary from 1 to 3 pounds. The effect of variation in contact pressure in measuring threads 
of fine pitches is indicated by the difference in readings obtained with 2 and 5 pounds pressure on a 24-pitch thread plug gage. The reading over the wires with 5 pounds pressure was 0.00013 inch less than with 2 pounds pressure.

Measurements of a thread plug gage made in accordance with these instructions, with wires which conform to the above specifications, should be accurate to $0.0001 \mathrm{inch}$. If the diameters of the wires are known only to an accuracy of 0.0001 inch, an accuracy better than 0.0003 inch in the measurement of pitch diameter can not be expected.

\section{MEASUREIMENT OF PITCH DIAMETER OF NATIONAL STRAIGHT THREADS}

The general formula for determining the pitch diameter of any thread whose sides are symmetrical with respect to a line drawn through the vertex and perpendicular to the axis of the thread, in which the very slight effect of helix angle is not taken into account, is: ${ }^{15}$

in which

$$
E=M+\frac{\cot a}{2 n}-G(1+\operatorname{cosec} a)
$$

$$
\begin{aligned}
E & =\text { pitch diameter } \\
M & =\text { measurement over wires } \\
a & =\text { one-half included angle of thread } \\
n & =\text { number of threads per inch } \\
G & =\text { diameter of wires. }
\end{aligned}
$$

This formula differs from those given in most engineering handbooks in that the latter, as generally given, yield a result which should check with the major diameter of the screw measured, while the pitch diameter itself is not mentioned. For a $60^{\circ}$ thread of correct angle and thread form this formula simplifies to:

$$
E=M+\frac{0.86603}{n}-3 G
$$

For a given set of best-size wires

when

$$
E=M-X
$$

$$
X=G(1+\operatorname{cosec} a)-\frac{\cot a}{2 n}
$$

The quantity $X$ is a constant for a given thread angle, and, when the wires are used for measuring threads of the pitch and angle for which they are the best size,

15 The general formula, in which the helix angle is taken into account, is:

$$
E=M+\frac{\cot a}{2 n}-G\left(1+\operatorname{cosec} a+\frac{S^{2}}{2} \cos a \cot a\right)
$$

in which $S=$ tangent of the helix angle.

The value of $S$, the tangent of the helix angle, is given by the formula:

in which

$$
S=\frac{L}{3.1416 E}=\frac{l}{3.1416 N E}
$$

$$
\begin{aligned}
L & =\text { lead } \\
N & =\text { number of turns per inch } \\
E & =\text { nominal pitch diameter. }
\end{aligned}
$$

In commercial practice the term $\left(\frac{G S^{2}}{2} \cos a \cot a\right)$ is neglected, as its value is small, being in all cases less than 0.00015 inch for standard fastening screws when the best-size wire is used, and the above formula takes the simplified form given above. This practice is permissible provided that it is uniformly followed, and in оrder to maintain uniformity of practice, and thus avoid confusion, the Bureau of Standards uses the latter formula except when the value of the term $\left(\frac{G S^{2}}{2} \cos a \cot a\right)$ exceeds 0.00015 inch, as in the case of Acme and multiple threads, or other threads having exceptionally large helix angles. 
the pitch diameter is obtained by the simple operation of subtracting this constant or factor from the measurement taken over the wires. In fact, when best-size wires are used, this factor is changed very little by a moderate variation or error in the angle of the thread. Consequently, the factors for the various sets of wires in use may be tabulated, thus saving a considerable amount of time in the inspection of gages. However, when wires of other than the best size are used, this factor changes quite appreciably with a variation in the angle of the thread.

It has been shown that, with the exception of coarse pitch screws, variation in angle from nominal value causes no appreciable change in the quantity $X$ for the best-size wires. On the other hand, when a wire near the maximum or minimum allowable size is used, a considerable change occurs, and the values of the cotangent and cosecant of the actual measured half angle are to be used. It is apparent, therefore, that there is a great advantage in using wircs very closely approximating the best size. For convenience in carrying out computations, the values of $\frac{\cot a}{2 n}$ for standard pitches are given in Table 88 .

\section{MEASUREMENT OF PITCH DIAMETER OF NATIONAL TAPER THREADS}

The pitch diameter of a taper thread plug gage is measured in much the same manner as that of a straight thread gage, except that a definite position at which the measurement is to be made must be located. A point at a known distance $L$ from the end of the gage is located by means of a combination of precision gage blocks and the cone point furnished as an accessory with these blocks, as shown in Figure 39 at $A$. The gage is set vertically on a surface platc, the cone point is placed with its axis horizontal at the desired height, and the plug is turned until the point fits accurately into the thread. The position of this point is marked by placing a bit of Prussian blue or wax immediately above it. Measurement is made over the wires in the usual manner, but care must be taken that the contact surfaces of the micrometer make contact with all three wires, since the micrometer is not perpendicular to the axis of the screw when there is proper contact. See Figure 39. On account of this inclination, the measurement over the wires must be multiplied by the secant of the half angle of the taper of the thread. The formula for the pitch diameter of any taper thread plug gage, the threads of which are symmetrical with respect to a line perpendicular to the axis, then has the form: ${ }^{16}$

$$
E=M \sec y+\frac{\cot a}{2 n}-G(1+\operatorname{cosec} a)
$$

in which

$$
\begin{aligned}
E & =\text { pitch diameter } \\
M & =\text { measurement over wires } \\
y & =\text { half angle of taper of thread } \\
n & =\text { number of threads per inch }=1 / p \\
a & =\text { half angle of thread } \\
G & =\text { diameter of wires. }
\end{aligned}
$$

Thus the pitch diameter of a "national (American Briggs') standard pipe thread gage" having correct angle $\left(60^{\circ}\right)$ and taper $(3 / 4$ inch per foot) is then given by the formula:

$$
E=1.00048 M+0.86603 p-3 \mathrm{G}
$$

\footnotetext{
16 See footnote 15.
} 
The pitch diameter at any other point along the thread, as at the gaging notch, is obtained by multiplying the distance parallel to the axis of the thread, between this point and the point at which the measurement was taken, by the taper per inch, then adding the product to or subtracting it from the measured pitch diameter according to the direction in which the second point is located with respect to the first.

The following method, illustrated in Figure 40, has a theroretical advantage over the first method in that it is independent of the taper of the thread, and, therefore, requires less computation; or if the taper is not measured but assumed to be correct, it is more accurate. The axis of the gage and the line of

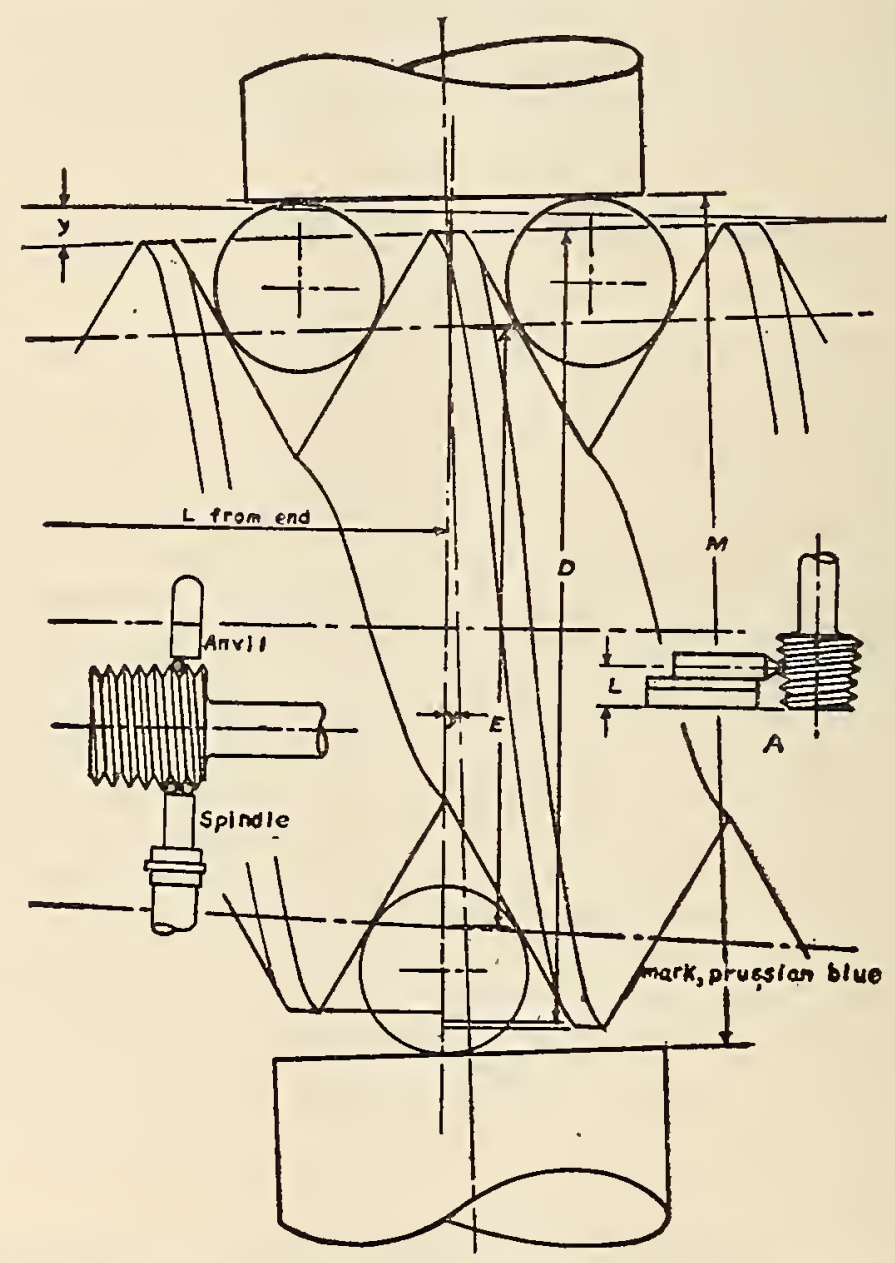

FIG. 39.-Measurement of pitch diameter of taper thread gages by the three-wire method

measurement are constrained perpendicular to each other. This is easily done on a measuring machine if the gage is supported on centers mounted on a slide whose ways are perpendicular to the line of measurement. If a micrometer caliper is used, its spindle is constraincd perpendicular to the axis of the screw. One method is to place the gage on a surface plate with its axis vertical, and support the micrometer in a horizontal position with its anvil and spindle resting on two equal combinations of gage blocks as shown in Figure 40 at $A$. A single wire is inscrted in the thread at the point located as in the previous method, and one other wire is placed in the upper thread on the opposite side. A measure- 
ment is taken over the two wires; the second wire is then moved to the thread immediately below, and a second reading is taken. The mean of these two readings is substituted in any of the above formulas.

\section{MEASUREMENT OF PITCH DIAMETER OF THREAD RING GAGES}

The application of direct methods of measurement to determining the pitch diameter of thread ring gages presents serious difficulties, particularly in securing

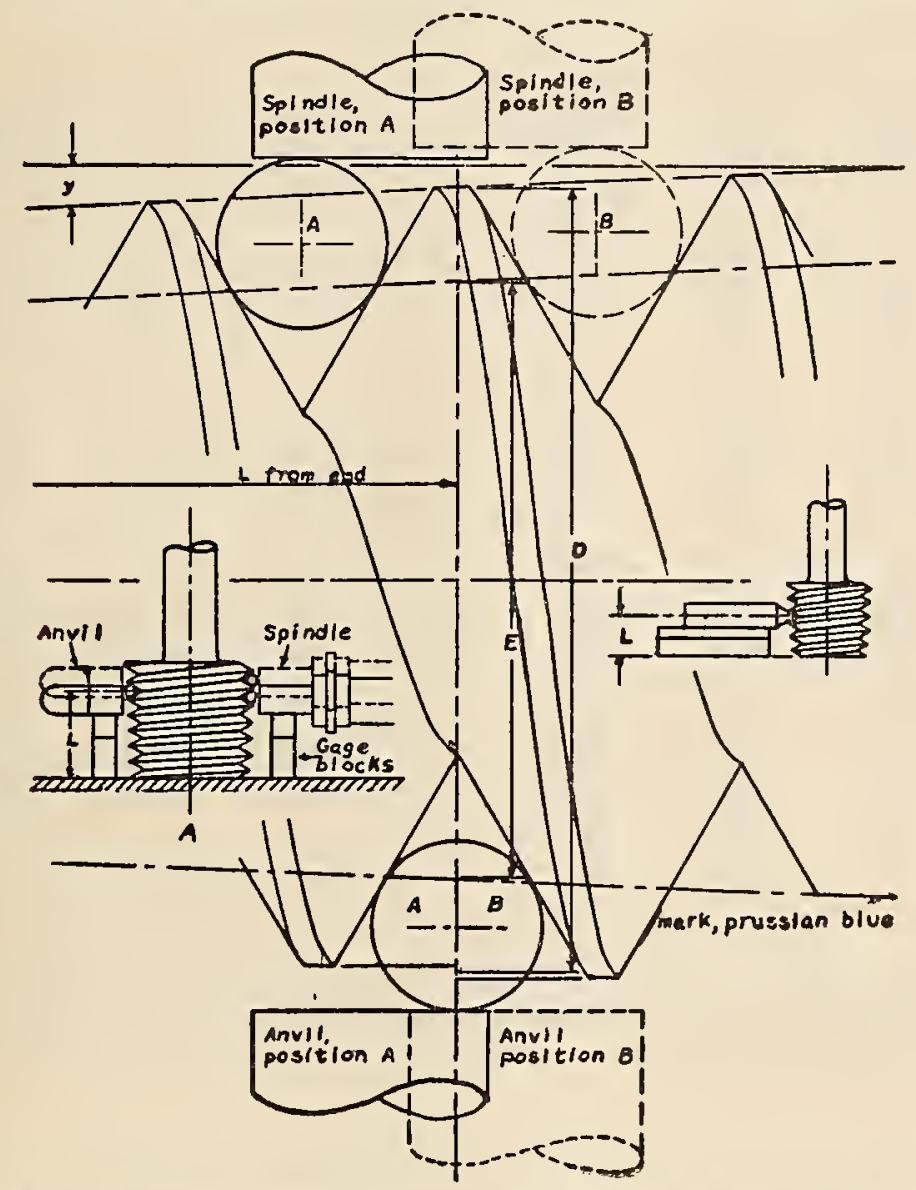

FIG. 40.-Measurement of pitch diameter of taper thread gages by the two-wire method

proper contact pressure when a high degree of precision is required. The usual practice is to fit the ring gage to a master setting plug. When the thread ring gage is of correct lead, angle, and thread form, within close limits, this method is quite satisfactory and represents standard American practice. It is the only method available for small sizes of threads. For the larger sizes, various more or less satisfactory methods have been devised, but none of these have found wide application. 
TABLE 88.-Wire sizes and constants, national coarse and fine threads, and national (American Briggs') pipe threads

\begin{tabular}{|c|c|c|c|c|c|c|}
\hline \multicolumn{3}{|c|}{ Wire sizes 1} & \multirow{2}{*}{$\begin{array}{l}\text { Threads } \\
\text { per inch } \\
\quad n\end{array}$} & \multirow{2}{*}{$\begin{array}{l}\text { Pitch } \\
p=\frac{1}{n}\end{array}$} & \multirow{2}{*}{$\frac{\text { Pitch }}{2}$} & \multirow{2}{*}{$\begin{array}{l}\text { Depth of } \\
\text { V thread } \\
\frac{\cot 30^{\circ}}{2 n}\end{array}$} \\
\hline $\begin{array}{c}\text { Best } \\
0.577350 p\end{array}$ & $\underset{1.010363 p}{\text { Maximum }}$ & $\begin{array}{l}\text { Minimum } \\
0.505182 p\end{array}$ & & & & \\
\hline 1 & 2 & 3 & 4 & 5 & 6 & $y$ \\
\hline $\begin{array}{l}\text { Inch } \\
0.00722 \\
.00802 \\
.00902 \\
.01031 \\
.01203\end{array}$ & $\begin{array}{c}\text { Inch } \\
0.01263 \\
.01403 \\
.01579 \\
.01804 \\
.02105\end{array}$ & $\begin{array}{c}\text { Inch } \\
0.00631 \\
.00702 \\
.00789 \\
.00902 \\
.01052\end{array}$ & $\begin{array}{l}80 \\
72 \\
64 \\
56 \\
48\end{array}$ & $\begin{array}{c}\text { Inch } \\
0.01250 \\
.01389 \\
.01562 \\
.01786 \\
.02083\end{array}$ & $\begin{array}{c}\text { Inch } \\
0.00625 \\
.00694 \\
.00781 \\
.00893 \\
.01042\end{array}$ & $\begin{array}{l}0.01083 \\
.01203 \\
.01353 \\
.01546 \\
.01804\end{array}$ \\
\hline $\begin{array}{l}.01312 \\
.01443 \\
.01604 \\
.01804 \\
.02062\end{array}$ & $\begin{array}{l}.02296 \\
.02526 \\
.02807 \\
.03157 \\
.03608\end{array}$ & $\begin{array}{l}.01148 \\
.01263 \\
.01403 \\
.01579 \\
.01804\end{array}$ & $\begin{array}{l}44 \\
40 \\
36 \\
32 \\
28\end{array}$ & $\begin{array}{l}.02273 \\
.02500 \\
.02778 \\
.03125 \\
.03571\end{array}$ & $\begin{array}{l}.01136 \\
.01250 \\
.01389 \\
.01562 \\
.01786\end{array}$ & $\begin{array}{l}.01968 \\
.02165 \\
.02406 \\
.02706 \\
.03093\end{array}$ \\
\hline $\begin{array}{l}.02138 \\
.02406 \\
02887 \\
.03208 \\
.03608\end{array}$ & $\begin{array}{l}.03742 \\
.04210 \\
.05052 \\
.05613 \\
.06315\end{array}$ & $\begin{array}{l}.01871 \\
.02105 \\
.02526 \\
.02807 \\
.03157\end{array}$ & $\begin{array}{l}27 \\
24 \\
20 \\
18 \\
16\end{array}$ & $\begin{array}{l}.03704 \\
.04167 \\
.05000 \\
.05556 \\
.06250\end{array}$ & $\begin{array}{l}.01852 \\
.02083 \\
.02500 \\
.02778 \\
.03125\end{array}$ & $\begin{array}{l}.03208 \\
.03608 \\
.04330 \\
.04811 \\
.05413\end{array}$ \\
\hline $\begin{array}{l}.04124 \\
.04441 \\
.04811 \\
.05020 \\
.05249\end{array}$ & $\begin{array}{l}.07217 \\
.07772 \\
.08420 \\
.08786 \\
.09185\end{array}$ & $\begin{array}{l}.03608 \\
.03886 \\
.04210 \\
.04393 \\
.04593\end{array}$ & $\begin{array}{l}14 \\
13 \\
12 \\
11.5 \\
11\end{array}$ & $\begin{array}{l}.07143 \\
.07692 \\
.08333 \\
.08696 \\
09091\end{array}$ & $\begin{array}{l}.03571 \\
.03846 \\
.04167 \\
.04348 \\
.04545\end{array}$ & $\begin{array}{l}.06186 \\
.06662 \\
.07217 \\
.07531 \\
.07873\end{array}$ \\
\hline $\begin{array}{l}.05773 \\
.06415 \\
.07217 \\
.08248 \\
.09623\end{array}$ & $\begin{array}{l}.10104 \\
.11226 \\
.12630 \\
.14434 \\
.16839\end{array}$ & $\begin{array}{l}.05052 \\
.05613 \\
.06315 \\
.07217 \\
.08420\end{array}$ & $\begin{array}{r}10 \\
9 \\
8 \\
7 \\
6\end{array}$ & $\begin{array}{l}.10000 \\
.11111 \\
.12500 \\
.14286 \\
.16667\end{array}$ & $\begin{array}{l}.05000 \\
.05556 \\
.06250 \\
.07143 \\
.08333\end{array}$ & $\begin{array}{l}.08660 \\
.09623 \\
.10825 \\
.12372 \\
.14434\end{array}$ \\
\hline $\begin{array}{l}.11547 \\
.12830 \\
.14434\end{array}$ & $\begin{array}{r}.20207 \\
.22453 \\
.25259\end{array}$ & $\begin{array}{l}.10104 \\
.11226 \\
.12630\end{array}$ & $\begin{array}{l}5 \\
4.5 \\
4\end{array}$ & $\begin{array}{l}.20000 \\
.22222 \\
.25000\end{array}$ & $\begin{array}{l}.10000 \\
.11111 \\
.12500\end{array}$ & $\begin{array}{l}.17321 \\
.19245 \\
.21651\end{array}$ \\
\hline
\end{tabular}

1 These wire sizes are based on zero helix angle. Also maximum and minimum sizes are based on a width of flat at the crest equal to $1 / 8 \times p$. The width of flat of "national pipe thread gages" is slightly less than this, so that the minimum size listed is slightly too small for such gages. In any case, the use of wires of either extreme size is to be avoided. 


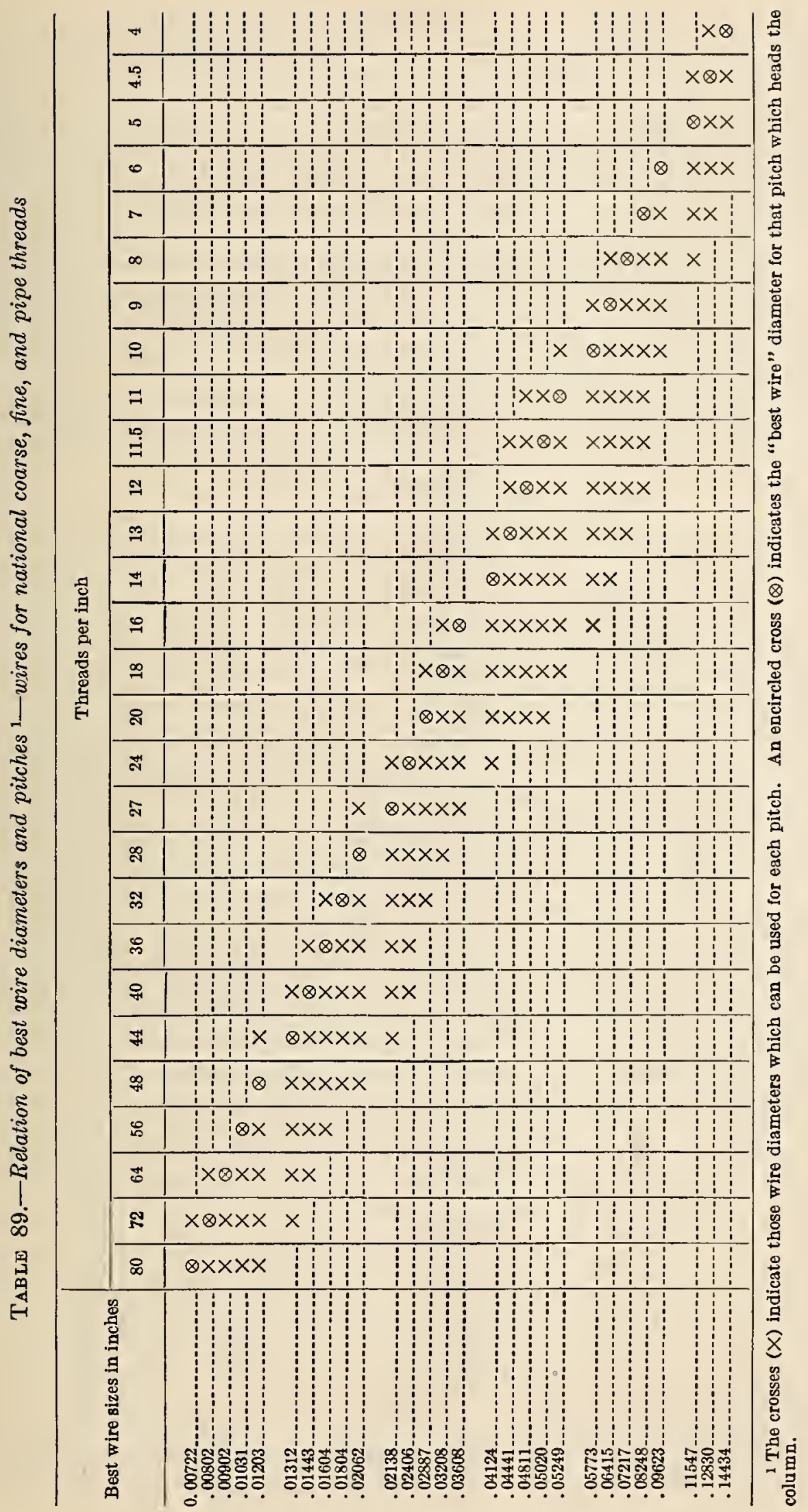




\section{APPENDIX 3. CONTROL OF ACCURACY OF THREAD ELE- MENTS IN THE PRODUCTION OF THREADED PRODUCT}

In order to maintain the dimensions of threaded product within the limiting sizes specified, it is essential that the tools used and the processes applied be suitable for the particular requirements. An analysis of the various factors controlling the accuracy of the individual thread elements is here presented. In this analysis, the fundamental factors controlling the accuracy of the elements of a screw thread are stated, and are followed by a brief discussion of the relationship of these factors to each of the prevailing commercial methods of producing screw threads. It is recognized, however, that certain varying factors are involved, such as lubrication, method of holding the work or tool, sharpness of cutting edges, etc., so that it is not always possible to predetermine the exact sizes of the tools required to accomplish the desired results.

Screw threads are usually produced either by cutting or rolling. Five general methods of cutting, two of rolling, and two of finishing screw threads are in common use.

\section{FUNDAMENTAL FACTORS}

The accuracy of the individual elements of a thread is controlled mainly as follows:

Angle by the angle between, and contour of the cutting edges of the tool used for cutting, or of the sides of the grooves of the die used for rolling.

Lead by the rate of the longitudinal motion of the tool with respect to the rate of revolution of the part to be threaded.

Major diameter of external thread by the outside diamcter of the stock, or by the forming tool.

Minor diameter of internal thread by the diameter of the hole in the work before threading. In the case of a drilled hole, this depends on the diameter and accuracy of grinding of the tap drill used.

Pitch diameter by the radial setting of the forming surface of the tool.

Thread form by the form and position of the tool, and the conditions under which it is used.

Inspection of the angle and proflle of the thread-forming tool is essential to control the accuracy of the thread produced. The same means and methods can be applied to such inspection as are applied in the measurement of screw thread gages and threaded product. Attention is directed to the optical projection apparatus for mcasuring angle and lead, and examining profile; the microscope, which may be readily adapted to shop requirements; and indicating gages, which may be designed to check the dimensions of threading tools.

The sources of lead errors require special consideration and for this purpose the methods of producing screw threads may be considered under two headings, namely, those in which relative longitudinal motion of the tool and product is controlled by means of a lead screw, and those in which the tool is self-leading.

(a) Tool Controlled By Lead Screw.-In cutting a thread on a lathe or other machine embodying a lead screw, using a single point cutting tool or single milling cutter, progressive lead errors are caused by (1) a progressive lead error in the lead screw; (2) lack of parallelism of the motion of the cutting tool, the axis of the lead screw, and the axis of the part to be thrcaded; and (3) incorrect ratio of the rate of revolution of the spindle to that of the lead screw, due to an incorrect or approximate combination of gears.

Local lead errors are caused by (1) local lead errors in the lead screw; (2) lost motion in the action of the lead screw or connecting mechanism; (3) varying frictional resistance in the mechanism; (4) when a live center is used, irregular 
play of its spindle in the bearings; and (5) variations in the amount of metal removed by the cutting tool.

Periodic lead errors are caused by (1) periodic lead errors in the lead screw; (2) eccentricity of motion of the lead screw; (3) thrust bearings of spindle or lead screw running out of true; (4) variations in the spacing of gcar teeth, or eccentric gears or mountings; (5) when a live center is used, eccentricity of motion of its spindle; and (6) periodic variations in the amount of metal removed, due to lack of uniformity of the material in diameter, straightness, or physical properties.

When a multiple-toothed threading tool is controlled by a lead screw, variations from correct spacing of the teeth of the tool are superimposed on the lead errors resulting from any of the above causes in that portion of the thread not passed over by every tooth of the tool. In the portion of the thread completely passed over by the tool, the effect of the difference in lead between the tool and lead screw is to produce a thin thread.

The simplest method of inspecting a machine tool to determine whether it will cut a screw thread within satisfactory limits is to cut carefully a sample screw on the machine and measure the lead errors of the screw. The obvious remedy for errors from such sources is the careful inspection of the various elements of the machine, and correction of the errors thus located, either by improving the design or by carefully refinishing or remaking the parts to a greater degree of accuracy.

(b) Self-Leading Threading Tool.-When a thread is cut by means of a tap or die, which, as ordinarily used, is self-leading and not controlled by a lead screw, lead errors may occur as the result of: (1) Incorrect lead of the tap or die; (2) too much or too little relief at the throat of the die or on the chamfer at the end of the tap; (3) the setting of an adjustable die or tap chaser to cut a thread considerably larger or smaller than that for which the tool was intended; that is, to cut a helix angle considerably different from the helix angle of the chaser; (4) excessive resistance to longitudinal motion; (5) improper alinement of the axis of the tap or die with that of the work, etc.; and (6) excessive angle relief.

The accuracy of the lead of the tap or of the chasers in the die is the most difficult of these sources of error to control, and indeed presents serious difficulties. There is, first, the difficulty of cutting a tap or chaser which is free from lead errors resulting from any of the causes outlined above; and second, the distortion which the steel composing the tap or die undergoes in hardening.

In the inspection of such thread forming tools, practically the same means and methods can be applied as in the measurcment of screw-thread gages. For checking the lead, indicating gages or some of the usual lead-measuring devices for screw-thread gages may be used. To measure the lead of a die chaser, the chaser must be held in a fixture in such a position that the direction of mcasurement corresponds to the direction of longitudinal motion of the chaser threads when cutting a thread.

\section{CUTTING OF SCREW THREADS}

(a) Single-Point Tool.-A screw thread may be produced by traversing a single-point threading tool-shaped to correspond to the shape of the thread space in an axial plane, and so placed as to cut an angle, equal to the angle of the top surface of the tool, in correct relation to the axis of the thread-along the revolving part to be threaded at such a rate as to produce a thrcad of the desired lead. This is the common method of cutting screws in an engine lathe; a lead screw driven by gearing being the thual means for imparting to the tool the longitudinal motion at the desired rate. This method is used commercially $16802^{\circ}-25 \uparrow-11$ 
only when special conditions make it necessary, as when the thread to be cut is not standard, or it is not practicable to apply other methods.

Various forms of single-point cutting tools for cutting threads of national form are illustrated in Figure 41 , at $A, B, C$, and $D$. The circular tool shown at $C$ has the advantage that it can be reground indefinitely without destroying its correct form. The diagram at $D$ shows the method for calculating the angle $x$ of the cutting tool, having a clearance angle $v$, in a plane perpendicular to the edge $M N$; and the formula for determining the clearance angle $v$, of a tool for cutting a thread of helix angle $s$, is also given. Such tools usually consist of hardened tool steel, ground to the correct form after hardening; special alloys such as "stellite" are also used for this purpose.

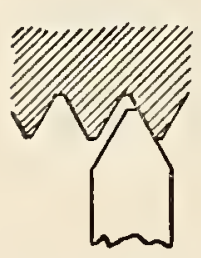

A

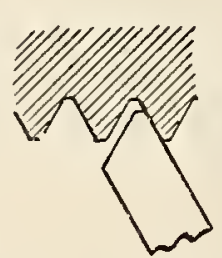

B

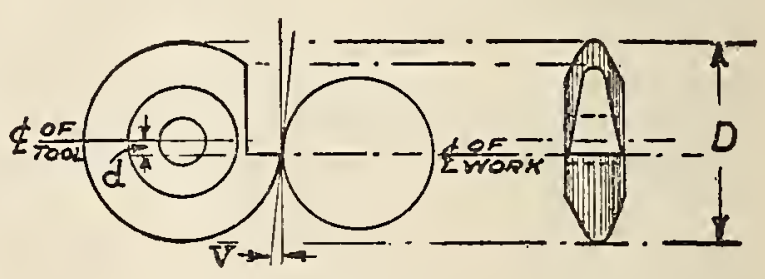

$d=\frac{D}{2} \sin V$
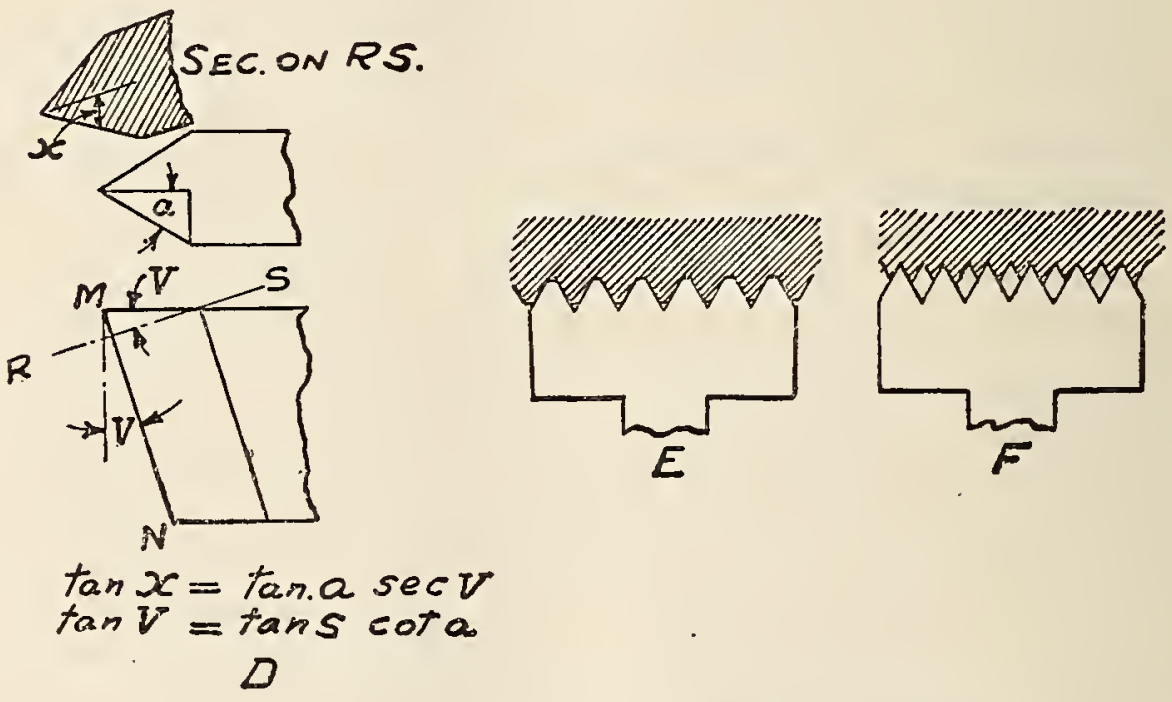

FIG. 41.-Single point and multiple point thread cutting tools

(b) Thread Chaser.-A screw thread may be produced by successively traversing a multiple-point thread tool, known as a chaser, along the part to be threaded, each tooth following in the thread in the same manner as a single-point thread tool. Two forms of chasers arc shown in Figure 41 at $E$ and $F$, the one at $F$ being especially suitable for cutting fine threads. Chasers are well adapted to roughing out threads, as they cut rapidly, and may be used for finishing threads accurately if the teeth are ground after hardening.

(c) TAP or Die.-A screw thread may be produced by using a tap for internal threads or die for external threads. These tools occur in considerable variety in their commercial forms, but consist essentially of a number of multiple-point cutters or chasers, usually four, arranged circumferentially. They may be 
either solid or adjustable, and collapsible or self-opening, respectively, for withdrawing quickly from the work after threading. By their use a thread is generally finished by one passage of the tool, although a second or finishing cut is sometimes made to secure greater accuracy. Dies are applied, in general, to threading screws, bolts, and studs; and taps to nuts or other internal threads within the usual range of sizes. They are also applied to the threading of pipe and pipe fittings. The rapidity with which threading operations may be performed by the use of taps and dies, within the limits of accuracy suitable for a large percentage of commercial work, makes them most efficient and widely used threading tools. It is only in cutting large sizes or coarse pitches, or where a high degree of accuracy is desired, that their use may be less economical than other means of cutting threads.

Aside from lead errors, which have been previously considered, the accuracy of the thread produced depends on the form of the cutting teeth, character of the cutting edges, clearance or relief for cutting edges, construction of the tool, and the conditions under which it is used.

(d) Milling Cutter.-A screw thread may be produced by feeding in to the depth of the thread and then traversing a rapidly revolving single milling cutter along the slowly revolving part to be threaded at such a rate as to produce a thread of the desired lead; the pronle of the cutting edges of the cutter conforming approximately to the shape of the thread groove in an axial plane, and the axis of the cutter being set at an angle to the axis of the thread, in a plane parallel to the axis of the thread, equal to the mean helix angle of the thread cut. The single-cutter method of thread milling is especially applicable to the cutting of large threads of coarse pitch, multiple threads, and the heavier classes of work. When the amount of metal to be removed is large, as compared with the size of the screw, this method is especially suitable because the torsional strain is much smaller than that produced by a die, and consequently the accuracy of the screw produced is greater. ${ }^{17}$

(e) Threading Hob.-A screw thread may be produced by feeding in to the depth of the thread, and then traversing a rapidly revolving multiple milling cutter or thread hob, somewhat longer than the length of the thread to be cutwhich consists of annular rows of teeth, whose centers lie in planes perpendicular to the axis of the cutter (in effect a series of single cutters formed into one solid piece), and the axis of which is parallel to the axis of the thread-along the slowly revolving part to be threaded slightly more than either one or two complete revolutions of the work, at a rate per revolution of the work equal to the pitch of the thread. The multiple-cutter method of thread milling is used largely for cutting comparatively short threads, usually of fine or medium pitches, when smoothness or a considerable degree of accuracy is desired, or when the thread must maintain a fixed relation with a point or surface on the work.

The error introduced in the form of thread produced by cutter teeth having the same form as that of the intended form of thread, as the result of the axes of cutter and thread being parallel, is usually not serious except when the helix angle is large. ${ }^{18}$

\footnotetext{
17 For refinements in connection with the determination of the profile of cutting edge of a thread milling cutter, see "The milling of screw threads and other problems in the theory of screw threads," bF II. II. Jeffcott. Proceedings of the Institution of Mechanical Engineers, 1922-I, pp. 51j-528, and discussion pp. 529-562; or Engineering (London) 113, Apr. 7, 1922, pp. 411-442, and discussion pp. 412-414.

${ }^{18}$ For formulas which may be applied in such cases to determine and plot the exact contour of the cutting edges to produce, as nearly as possible, the thread form required, see "Side-cutting of thread milling hobs," by Earle Buckingham. Transactions of the American Society of Mechanical Engineers, 42, 1920, pp. 569-593; also the reference cited in foot note 17 for thread milling cutter profile.
} 


\section{ROLLING OF SCREW THREADS}

The second general process for forming screw threads, namely, that of rolling, is a cold-forging process. It may be defined as an impression or displacement method whereby the threads are formed by means of a die or roll having threads or ridges, which are forced into the material to be threaded, and, by displacing it, produce a thread of the required form and pitch. In this process no material is removed, but the metal is displaced from the thread space and forced up on each side above the original surface of the piece to be threaded. Thus, the major diameter of a V-shaped $60^{\circ}$ thread so produced is found in practice to be greater than the original diameter of the blank by an amount varying from 65 per cent of the single-depth of thread for small screws to 85 per cent for large screws. An approximate formula, based on geometrical considerations only, for the diameter of a blank to be threaded to national form is as follows:

in which

$$
D_{1}=\sqrt{D^{2}-1.3 D p+0.63 p^{2}}
$$

$$
\begin{aligned}
& D_{1}=\text { diameter of blank } \\
& D=\text { major diameter of thread } \\
& p=\text { pitch of thread. }
\end{aligned}
$$

In case the thread required must be accurate within close limits, the exact value of $D_{1}$ necessary in any given case must be determined experimentally, as its value is affected by the physical properties of the material. ${ }^{10}$

The thread-rolling process is the most rapid and economical method of forming screw threads in quantity production, when the part to be threaded is of such form as to permit its use. It is used only for external threads and is not regarded as being feasible for internal threads, since the area of contact of the roll in an internal thread is relatively much larger than on an external thread, and in order to displace the metal a very heavy pressure is required. It is difficult to support the work with the necessary rigidity to withstand the heavy pressure, and to provide a bearing for the roll which will withstand the strain.

Screw threads may be rolled by either of two methods, as follows:

(a) Threading Roll.-By forcing a cylindrical disk or roll, having a threaded periphery and being free to rotate on the pin or bolt on which it is mounted, against the piece to be threaded while the latter is revolving. The cylindrical roll is used when the work is in an automatic screw machine or turret lathe and it is impossible to cut the thread required by means of a thread cutting die, or when an additional operation would be necessary before cutting the thread. The thread on the roll corresponds in pitch, and approximately in form, to the thread to be rolled. The roll may be presented to the work in either a tangential direction as shown at $A$, Figure 42 , or radially as shown at $B$; a satisfactory thread is formed in either case.

(b) Thread Rolling Dies.-By rolling the blank between dies, which may be either flat or cylindrical in form, when performed by machines designed exclusively for this work. When flat dies are used, as shown in Figure 42 at $C$, one die $M$ remains stationary, and the other die, $N$, which is parallel or ncarly parallel to $M$, has a reciprocating movement. The faces of the dies have parallel milled or planed grooves of approximately the same form as that of the required thread which are set at an angle to the line of motion of the blank equal to the helix angle of

${ }^{19}$ This formula is derived in "Size of stock for holts having rolled threads," hy F. Webster. American Machinist, 30, Oct. 31, 1907, p. 630. 
the thread to be produced. The angles of the grooves and ridges in a plane perpendicular to the direction of the grooves are given by the formula:

in which

Tan $a_{1}=\tan a \cos s$

$$
\begin{aligned}
& a_{1}=\text { half angle of ridge of die } \\
& a=\text { half angle of thread to be rolled } \\
& s=\text { helix angle of thread. }
\end{aligned}
$$

The spacing of the ridges is determined by the formula:

in which

$$
p_{1}=p \cos s
$$

$$
\begin{aligned}
& p_{1}=\text { spacing of ridges of die } \\
& p=\text { pitch of thread to be rolled } \\
& s=\text { helix angle of thread. }
\end{aligned}
$$

The blank is inserted at one end of the stationary die, and rolls between the die faces until it is ejected at the other; the thread being formed in one passage of the blank. When cylindrical dies are used, one of the dies, which is a complete cylinder, revolves continuously in one direction and the other is a stationary cylindrical segment. This method is used extensively for threading almost all
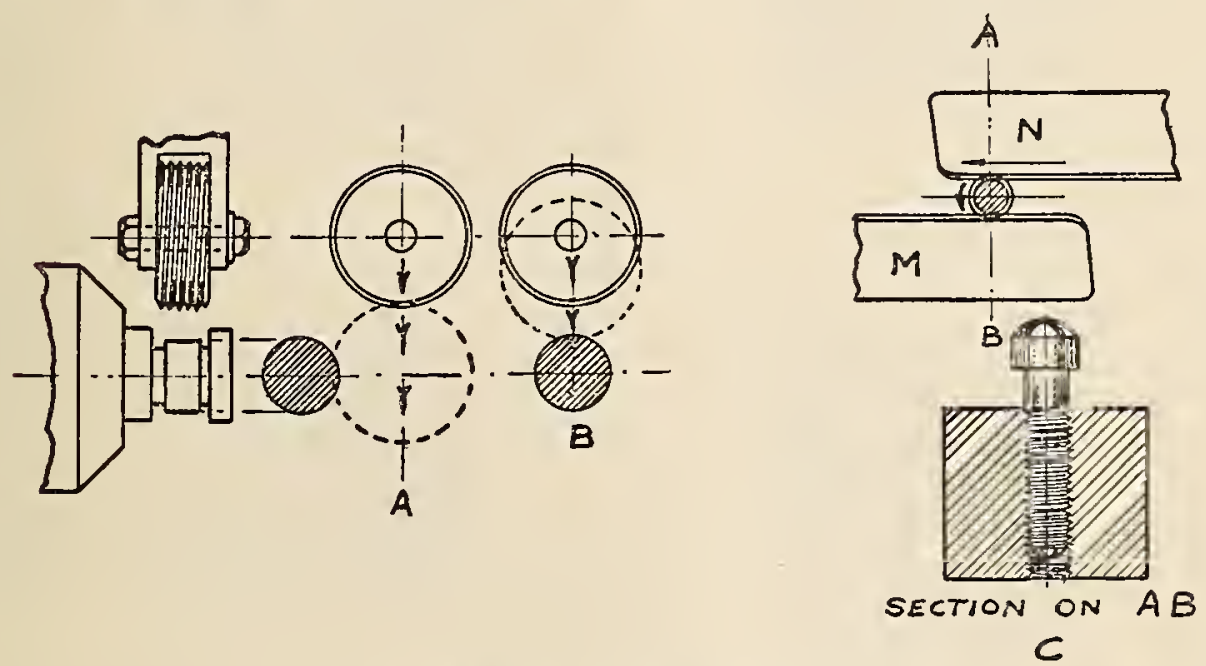

Fra. 42.-Methods of rolling screw threads

forms of small and medium sizes of screws and bolts, when required in suffciently large quantities to warrant the use of a thread rolling machine. ${ }^{20}$

\section{FINISHING OF SCREW THREADS}

On account of the difficulty of producing an accurately finished thread by means of a cutting tool, in ordinary gage-making practice the thread is lapped, or ground and lapped, in order to finish all elements of the thread to correct dimensions. The process of grinding is applied to hardened screns only, and is intended to correct any errors present as the result of distortion in the hardening process, as well as those resulting from the cutting operation. Lapping is usually applied to hardened screws threads, and may be either substituted for grinding, or performed after grinding to remove the marks left by the grinding wheel and to produce a smooth and highly-polished surface. These processes are used largely in the production of screw-thread gages.

${ }_{20}$ The principles involved in determining the spacing and angle of ridges of flat dies, and position of the dies, are considered in "Principles of thread rolling and the setting of dies," by J. F. Springer, American I.Iachinist, 33, Apr. 21, 1910, pp. 739-741. 
(a) Grinding.-The grinding of a thread is similar to the process of milling a thread by the single-cutter method. The profile of the periphery of the grinding wheel is "dressed" by means of a diamond to conform to the shape of the thread groove in an axial plane, with the axis of the wheel set at an angle to the axis of the thread, in a plane parallel to the axis of the thread, equal to the helix angle. In order to produce a thread having straight sides and correct angle, the periphery of the wheel should be dressed to the required angle after the wheel has been set to the helix angle, in the plane containing the axis of the thread and the center of the wheel. The same considerations as to the exact profile of the periphery of the grinding wheel, to produce a thread of exactly correct form, apply as for the tooth profile of a single milling cutter set at the helix angle of the thread. The principal differences between the thread milling and grinding processes are that a large diameter of grinding wheel is desirable, and several light cuts are taken, whereas, a small diameter of milling cutter is desirable and a single cut is taken.

(b) LAPping.-The lapping of a screw thread may be defined as a process of abrasion by successively traversing the thread, as it revolves, with a so-called lap, which consists of an engaging screw thread of softer material, usually finegrained cast iron, brass, or cold-rolled steel, in which very fine abrasive material is enbedded in the thread surface. For removing considerable material, the laps are charged with coarser abrasive, and for imparting a fine finish, a finer abrasive; in either case the abrasive used is very fine, and the lap is thoroughly lubricated. A number of laps may be necessary to finish either an internal or external thread to the required form and dimensions. The necessary laps for reducing the national form of thread are as follows:

(1) For reducing the sides of the thread, and this correcting the pitch diameter, the lap consists of a thread, of correct form, slightly large on all dimensions (about 0.001 inch) to go onto a plug, or small to go into a ring. The thread form of this lap is shown in Figure 43 at $A$. It is shorter in length than the thread to be lapped, in order that only local and periodic lead errors will be corrected and the lead over the entire length of the thread will not be affected.

(2) For reducing the major diameter of an external thread, or increasing the minor diameter of an internal thread, and correcting the form at the crest, a lap of full thread form may be used if the thread angle of the lap for an external thread is large (by about $2^{\circ}$ ), or for an internal thread is small, and proper clearances are provided on pitch and minor diameters.

(3) If the angle of the thread has been changed by improper lapping, or distorted by strains produced in hardening, it may be corrected by means of a lap of correct angle, with clearances at crest and root, shown at $C$, Figure 43.

(4) Local or periodic errors in pitch will have been corrected to a considerable extent by the above laps, but a progressive error in lead can only be corrected, if negative (short), by means of a lap of correct thread form whose lead is long; and if positive (long), by means of a lap whose lead is short. In either case the length of the lap should be about one and one-half times that of the thread to be lapped.

The order in which these laps are applied will depend on the nature of the errors in the work, and the changes which take place in the dimensions of the work during the operation. Frequent checking of the dimensions of the work is necessary to assure good results.

For sizing internally threaded laps, a hand tap, having a pilot whose diameter is equal to the minor diameter of the thread, tapered at the leading end threads, and accurately made to the dimensions of the finished thread is very convenient. It should be lubricated with a good grade of lard oil to impart a smooth finish to the threads in the lap. 


\section{APPENDIK 4. DESIGN AND CONSTRUCTION OF GAGES}

The following recommendations will be helpful in the design and construction of gages used in the inspection of threaded product (see also Section III division 6 , and fig. 22).

\section{MATERIAL}

The commission has no recommendations as to material to present. A body of manufacturers and users of gages, known as the Gage Stcel Committee, with headquarters at the Burcau of Standards, is developing such rccommendations. These will be based on results of an extensive experimental investigation now being earried on at the Bureau of Standards in cooperation with the Ordnance Department, United States Army, and various manufacturers.

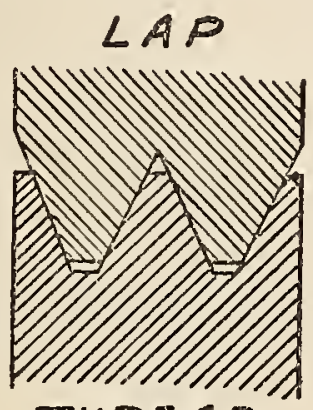

THREAD

$A$

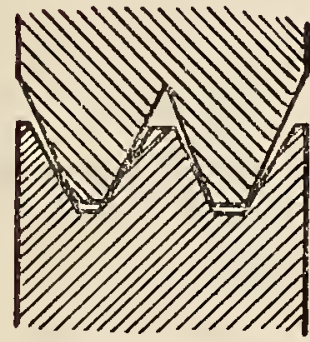

Frg. 43.-Thread form of laps for lapping screw threads

\section{PLUG THREAD GAGES}

(a) TYPE.-Thread plug gages $1 \frac{1}{2}$ inches and less in diameter should be made with a shank inserted in a handle and securely fastened.

Plug gages of more than $1 \frac{1}{2}$ inches in diametcr, unless otherwise specified, should have the gaging blank so made as to be reversible. This can be accomplished by having the finished hole in the gage blank fitting a shouldered projection on the end of the handle, the gage blank being keyed on and held with a nut.

Inspection and working thread plug gages with chamfered end threads should be provided with dirt grooves which extend into the gage for a depth of from one to four threads.

(b) Design of "Go" Thread Plug Gages.-The handle of the "go" thread plug gage should be chamfered in order to provide a ready means of identification of "go" gages as distingiushed from "not go" gages. The thread should be of basic form with the root clearcd to facilitate grinding and lapping.

The length of thread parallel to the axis of all standard "go" thread plug gages should be at least equal to the length of engagcment of the product. 
(c) Design of "Not Go" Thread Plug Gages.-All "not go" thread plug gages should be made to check the pitch diameter only. This necessitates removal of the crest of the thread, so that the dimension of the major diameter is never greater than that specified for the "go" gage, and also the removal of the portion of the thread at the root. - The crest of the thread should be truncated to to a depth corresponding to a flat equal to $p / 4$ and the groove be widened from a position one-fourth the depth of the sharp $\vee$ thread below the pitch diameter line to the bottom of the V-thread groove. This form of thread checks principally the pitch diameter and has sufficient gaging surface to provide against rapid wear.

\section{RING THREAD GAGES}

(a) TYPE.-All ring thread gages should be made adjustable. The thread should have the root cleared to facilitate grinding and lapping, and the crest truncated to correspond to the minor diameter of the internal thread of the product.

(b) Design of "Go" Thread Ring Gages.-The "go" gage should be distinguished from the "not go" gage by having a decided chamfer, and both gages should have their outside diameters knurled if made circular.

(c) Design of "Not Go" Thread Ring Gages.-All "not go" ring thread gages should be made to check the pitch diameter only. This necessitates removal of the crest of the thread so that the dimension of the minor diameter is never less than that specified for the maximum or "go" gage and also the removal of the portion of the thread at the root of the standard form.

\section{PLAIN PLUG GaGES}

Plain plug gages of $11 / 2$ inches and less in diameter should be made with a plug inserted in the handle and securely fastened. Plain plug gages of more than 11/2 inches in diameter should have the gaging blank so made as to be reversible. This can be accomplished by having a finished hole in the gage-blank fitting a shouldered projection on the end of the handle, the gage blank being held on with a nut.

The "go" plain plug gage should be distinguished from the "not go" gage by having a decided chanfer on the handle of the "go" gage.

\section{PLAIN RING GAGES}

Both the "go" and "not go" gages should have their outside diameters knurled if made circular.

The "go" gage should have a decided chamfer in order to provide a ready means of identification for distinguishing the "go" from the "not go" gage.

\section{PLAIN SNAP GAGES}

Snap gages may be either adjustable or nonadjustable. It is recommended that all snap gages up to and including one-eighth inch be of the built-up type. For larger snap gages, forge blanks, flat plate stock, or other suitable construction may be used.

Sufficient clearance beyond the mouth of the gage should be provided to permit the gaging of cylindrical work.

Snap gages for measuring lengths and diameters may have one gaging dimension only, or may have a maximum and minimum gaging dimension, both on one end, or on opposite ends of the gage. When the maximum and minimum gaging dimensions are placed on opposite ends of the gage, the maximum or "go" end of the snap gage should be distinguished from the minimum or "not co" end by having the corners of the gage on the "go" end decidedly chamfered. 


\section{APPENDIX 5. FUTURE WORK OF THE COMMISSION}

The following brief summary of the uncompleted projects to which the commission has devoted its attention is presented for the purpose of acquainting the public with the progress made, and of inviting the cooperation of all intercsted parties in bringing as much of this work as possible to a satisfactory conclusion.

While some of these projects are practically completed, others have assumed considerable proportions, on account of the variety of intercsts which must be considered, and some will undoubtedly require much time to complete. It is the intention of the commission to issue supplementary reports from time to time, as additional standardization projects are completed.

\section{SCREW THREADS USED IN ELECTRICAL INDUSTRY}

Three different forms of thread are in common use on electrical equipment, each type finding application on a certain class of work, but much confusion has existed as to their proper uses and combinations. Specifications have been formulated for the following types of screw threads: (1) Screw threads for conduit, and conduit couplings and fittings, (2) special straight pipe threads for fixtures, (3) fine threads for brass tubing, and (4) rolled threads for screw shells of electric sockets and lamp bases.

Data regarding item 2 are given in A. S. M. E. Bulletin No. 1525, report on "Straight Pipe Threads" of the Committee on Standardization of Special Threads for Fixturcs and Fittings. Data on item 3 arc given in a bulletin issued by the National Council of Lighting Fixture Manufacturers in 1921. Data on itcm 4 are given in A. S. M. E. Bulletin No. 1474, report on "Rolled Threads for Screw Shells of Electric Sockets and Lamp Bases" of the Committee on Standardization of Special Threads for Fixturcs and Fittings.

These specifications will be available in mimeographed form pending the publication of further sections of this report.

\section{BOLT AND NUT PROPORTIONS AND WRENCH OPENINGS}

Another project to which the commission has given attention is the standardization of bolt and nut proportions, particularly as to the widths across flats, because of the desirability of reducing the number of sizes of bar stock, dies, and wrenches necessary.

Several months after the commission undertook this work, the Sectional Committee on the Standardization of Bolt, Nut, and Rivet Proportions was organized under the procedure of the American Engineering Standards Committee with the Society of Automotive Engineers and the American Society of Mechanical Engincers acting as joint sponsors. To avoid duplication of effort and prevent the promulgation of two conflicting standards, a subcommittee of the commission and a subcommittee of the sectional committee have worked in close cooperation in developing proposed standards for wrench-head bolts and nuts.

The results of the work of these subcommittees were published late in $1923 \mathrm{by}$ the sponsors, as a tentative report of subcommittee No. 2 of the sectional committee entitled "Proposed Standard Sizes of Wrench Head Bolts and Nuts," for the purpose of obtaining criticisms and comments of all interested manufacturers and users, before the subcommittee made its final report to the sectional committee. The proposed sizes are intended to supersede all existing standards of wrench head bolts and nuts, of which a large number have grown up in various industries and sections of the country. As the result of the adoption of such standard series of sizes, there will be a marked saving in the number of sizes of bar stock and of finished bolts and nuts which the manufacturers and dealers must carry, and in the number of wrenches which the consumer requires to fit articles produced by the different industries. 
In fixing tolerances on widths across flats of bolt heads, nuts, and wrenches, it was intended that a minimum clearance be provided which would assure a fit of the wrench to the bolt head and nut, and a maximum clearance which would not permit the rounding of the corners of a hexagonal nut or bolt head nor spreading of the wrench jaws.

\section{MACHINE SCREW AND STOVE BOLT PROPORTIONS}

As in the standardization of bolt and nut proportions and wrench openings, the same subcommittee of the commission and a subcommittee of the Sectional Committee on the Standardization of Bolt, Nut, and Rivet Proportions have worked in cooperation to develop standard dimensions for machine-screw heads. The proposed standard sizes were published in March, 1924, by the sponsor bodies as a tentative report of subcommittee No. 3 of the sectional committee entitled, "Proposed Standard Sizes of Slotted Heads for Machine and Wood Screws." This report is being submitted to all interested manufacturers, dcalers, and users of these products for criticism and comment. This report contains head sizes for a number of machine screw sizes which arc not a part of the national coarse or national fine thread serics. The commission recommends that these sizes be eliminated.

There has been considerable difficulty in arriving at a satisfactory standard for stove bolt proportions on account of the difference in the types of manufacturing equipment used by Eastern and Western manufacturers. This difficulty is discussed in the above-mentioned report of subcommittee No. 3. Although the commission has not come to an agreement on head dimensions for stove bolts, it recommends that thread diameters and pitches conform to the specifications giren in Section III of this report for the national coarse thread series, class 1 , loose fit.

\section{OIL-WELL CASING THREADS}

One of the special problems brought to the attention of the commission was the great need for standardization of oil-well casing threads. Definite work toward such standardization was initiated by the Mid-Continent Oil and Gas Association, in 1921, but this was complicated by a proposal to simplify casing sizes and weights, and provide new standard sizes of nesting casing required for the deeper well drilling which is now necessary. Certain manufacturers have also endeavored to come to an agreement on thread standards, and a further influence toward that end has been the fostering of standardization of oil-field equipment by the American Petroleum Institute, and by Purchasing Agents' Associations.

Aside from the variety of casing sizes, further complications arise from the fact that three tapers and three or four pitches have been in use. Through the cooperative efforts of the American Petroleum Institute, the Standardization Committee of the Mid-Continent Oil and Gas Association, and the commission the standardization of casing threads has progressed to the point where certain agreements as to pitches and tapers have been effected. On the larger sizes 8 threads per inch, and on the smaller sizes, 10 threads per inch will be standard, with the dividing line at about 8 inches diamater of casing. The taper on the larger sizes will be three-fourths inch per foot, and on the smaller sizes threeeighths inch per foot, the change in taper and in pitch taking place at the same point. The American Petroleum Institute has determined upon a series of casing sizes which will meet present requirements, and only minor details in the specifications remain to be developed.

The standardization of oil-well casing and casing threads has an important bearing on the national defense, and for this reason the commission is very much gratified with the progress made by the industry in such standardization. 


\section{WRENCH FIT OF THREADED STUDS}

As announced in the 1921 report, insufficient data were available for the proper specification of tolerances and allowances (interferences) for class 5 , wrench fit. The commission, in cooperation with the Bureau of Standards, has since collected and compiled much information as to stud fits from technical literature and by correspondence with manufacturers of automobiles, trucks, gas and steam engincs, tractors, and other types of machinery in which studs are used. While the information thus obtained was very complete and valuable, the commission feels it necessary to obtain additional experimental data as to safe and proper limits for such interierences, on account of the wide variations in commercial practice as to maximum and mininum interferences.

The Bureau of Standarcis is conducting a series of tests, and has already obtained considerable data, in which the relations between pitch diancter interference, thread form, and assembling torque for representative sizes and pitches are being determined. The results of a somewhat similar series of tests also have been submitted by a large automobile manufacturer. As a result of this work the commission expects to be in a position to make definite recommendations in the not far distant future.

\section{WIRE GAGES, AND STOCK SIZES OF WIRE, METAL SHEET, AND PLATE}

The interest of the commission in sizes of stock for screws, and bolts, and in tap-drill sizes, led to an investigation of the possibilities for the simplification of wire gages. The existing confusion in the industries due to the many wire and sheet metal gages in use is well known. One of the causes of uncertainty arising from the multiplicity of gages in use is the failure on the part of a purchaser to designate a particular wire gage or to state the decimal size corresponding to the gage number; also errors frequently occur in transmitting this information. A further complication arises from the fact that, in common trade practices, different gages are used for different materials. The result is that, if, for examplc, a purchaser who has been accustomed to using a No. 10 steel wire for a certain purpose should order a No. 10 copper wire for the same purpose, he would receive a wire of a different diameter. Alleged sharp and dishonest practices arising from this situation have been brought to the attention of the Department of Commerce.

In the course of its investigation, the commission held a hearing on October 8, 1923 , in New York, N. Y., to consider the question. From the discussion at this hearing and the letters received, the commission has arrived at the following conclusions:

The following gages are in customary use in the United States:

\section{Designation of gage}

American Wire, or Brown \& Sharpe _ Copper, brass, bronze, and other nonferrous wires and sheets.

Steel Wire, or Washburn \& Moen.... Steel wire (other than music wire and drill rod).

Birmingham, or Stub's Iron Wire.... Telegraph and telephone wire, and to some extent thickncss of nonferrous sheets, and wall thickness of tubing.

Twist drill and steel wire Twist drills and drill rods.

U. S. Standard for sheets and plates_- Sheet and plate iron and steel.

Music wire. Piano and other music wire.

Sheet zinc. 
From the testimony given before the commission, it appears that while there is a certain amount of overlapping of the various gages used on various materials and in different industries and much uncertainty on the part of the public, the opinion in each industry is that the particular gage used in that industry is reasonably satisfactory, and that all other industries should adopt that particular gage. In view of this attitude on the part of those who use gages, it is the opinion of the commission that it would be impracticable to attempt to remedy the present situation by recommending the adoption of any one of the various existing gages and the elimination of all others. It is further the opinion of the commission that a more practical solution would be to eliminate all of the arbitrary gage series and to express all diameters and thicknesses of wires, drill rods, plates, and sheets in terms of decimal parts of an inch.

The designation of diameters and thicknesses of wires and sheets in decimal fractions of an inch instead of by gage numbers does not necessarily involve any change from present practice so far as actual dimensions are concerned. Each industry would naturally continue the use of present stock sizes. A point which should not be overlooked, however, is the possibility of selecting stock sizes for each material or industry such that the stock sizes for all materials and industries would form a consistent and logical series. A part of this series would be employed in one industry or on one type of material, while other parts would be used on other materials or in other industries. It seems probable that judicious selection of stock sizes in the various materials and industries would make possible a very considerable reduction in the quantity of materials carried in stock, and in the number and variety of dies used in the production of drawn material, by eliminating certain sizes from the various gage series which are unnecessarily close together. This presents a problem in simplification by elimination, the solution of which the commission is not in a position to undertake.

\section{ACME THREADS}

The sizes and pitches of Acme threads in use are so extensive in variety that an effort to evolve a standard series has been considered futile. The table given below was compiled from 34 replies to questionaires sent to machinery manufacturers and shows the relative percentage of each pitch used in screws for transmitting power, and those diameters corresponding to the various pitches most often used. Both right and left handed single threads are included. This table, however, can not be considered as covering the entire field.

TABLE 90.-Diameters and pitches of Acme threads commonly used

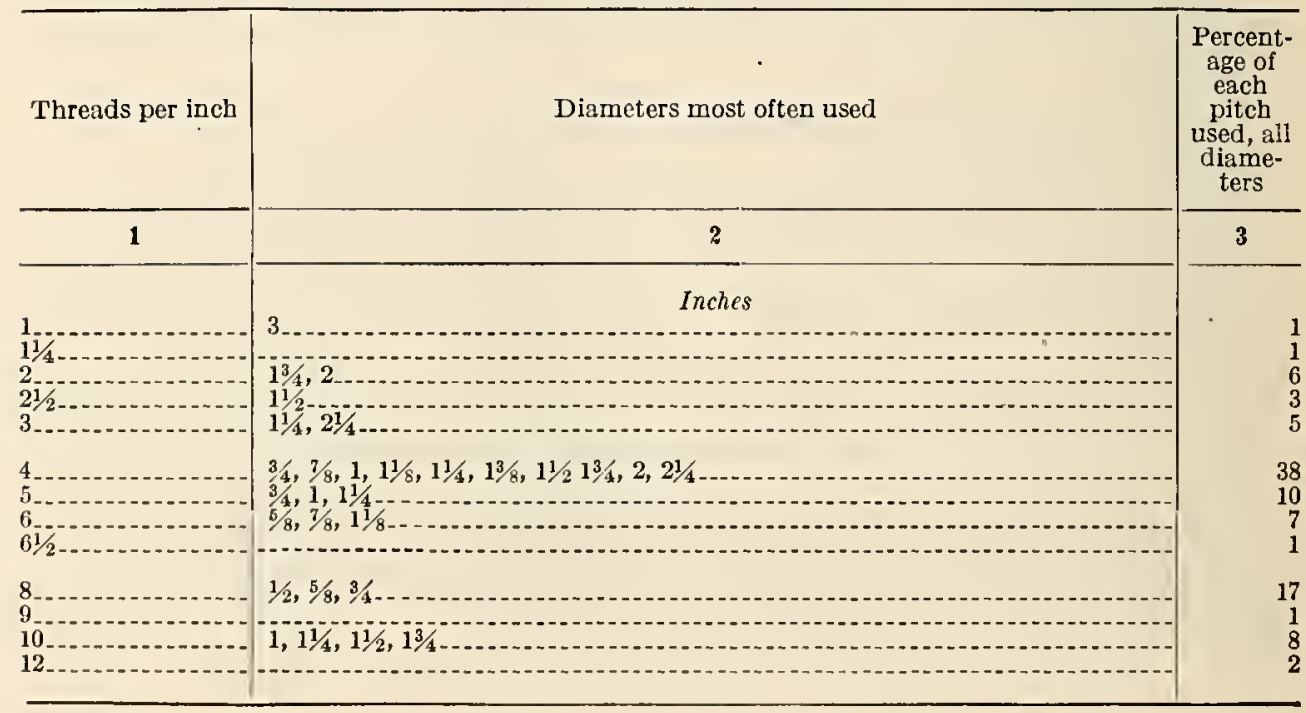




\section{OTHER STANDARDIZATION PROJECTS}

(a) Tolerance Specifications for Pipe Threads Subject to High PressuRes.-The requirements of taper threads for high-pressure work are not as generally realized as they should be, and attempts are frequently made to use commercial pipe threads, made to the usual tolerance specifications, with very unsatisfactory results. An investigation as to tolerances on the individual thread elements of pipe threads for such purposes is proposed.

(b) Instrument Tubing Threads.-The lack of a universal standard for threads on brass tubing for instrument work is not as important a question from the standpoint of the general public as standard threads for tubing for domestic purposes, for which specifications will be given in the section on screw threads used in electrical industry. Nevertheless, it would be very desirable to have definite recommended pitches for various sizes of instrument tubing. The French have considered the matter of suffeient importance to formulate a definite standard for threaded brass tubes for optical instruments, which was adopted in December, 1916. Very little information is available as to Ancrican practice, but standards are used for certain special purposes, such as threads on miscroscope objectives, which are to a large extent interchangeable.

(c) Threads on Instroment Screws.--Smaller sizes and finer pitches of screws than those provided in the national coarse and national fine thread series, together with the latter, are extensively used in instrument work. Various manufacturers' standards for the small sizes are in use but there has been no concerted effort among American manufacturers to adopt a common standard.

(d) Form of Thread for Valve Stems.-From the points of view of the threading tool and valve manufacturers, it would be desirable to standardize the form of thread used on valve stems. A variety of forms are now in use, principally the truncated $60^{\circ}$ thread and modified Acme thread.

(e) Threads on Condenser Tube Ferrules. - It has been brought to the attention of the commission that several different pitches are used on the same nominal sizes of condenser tube ferrules. This is a source of inconvenience and expense in making repairs.

(f) Plumbers' Fine Threads. - Fine pitch taper threads on thin-walled brass tubing are being produced to various plumbing manufacturers' standards. A unification of these standards is desirable.

(g) Special Threads.-Other threads in use in the United States, for which standard specifications may be formulated are the Buttress Thread, Harvey Grip Thread, Knuckle Thread, Square Thread, etc. 



\section{INDEX}

Page

Accuracy, control of

Allowance (definition)

(numerical values)

Angle errors, diameter equivalent of .. 24 ,

$28,32,33,92,112,136,146$

Angle of helix (see Helix angle) .............. 14 thread

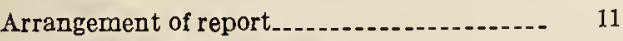

Authorization hy Congress.........-......-... 5 5

Axis of screw . ............. 14

Base of thread.................................. 14

Basic size-15

Bolt and nut proportions...-.............-. 165

Bolts and nuts, screw threads for

Chaser, thread.

Class A (ft)

Class B

Class C

Class D....................... 97

Class $\mathrm{E}_{\text {- }}$

Class 1 , loose fit. . ........................ 21, 23, 37, 41

Class 2, frce fit........................... 21, 27, 38,42

Class 3 , medium fit.................... 22, $30,39,43$

Class 4, close fit ....................... 22, 32, 40, 44

Class 5, wrench fit_............................ 22, 167

Classification of fits...-.................. 21, 23, 90

Clearance, crest

at major diamcter...................... 18

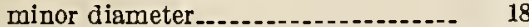

Close fit, class 4 .................... 22, 32, 40, 44

Committees, suh............................ 8

Comparators, thread ................... 71

Core diametcr (see Minor diamcter) .......... 13

Crest......................................... 14 clearance............................... 15

Cutter, thread milling

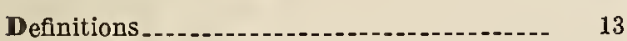

Depth of engagement.........-.............. 14 thread.-...-............................ 14, 64

Direction of tolerances on gages.......-..-.- 69

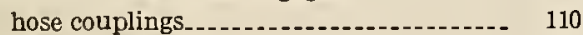

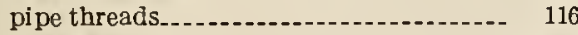

screw and nut.................... 22, 89

Engagement, depth of length of

Errors, control of lead . ..................... 156-157 diameter equivalents of lead and angle .- 24, $28,32,33,92,112,136,146$

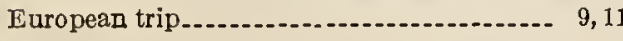

Finish

Fit

Form of thread:

fire-hose coupling threads.-...-........... 108

hose-coupling threads....................- 108

national coarse and fine threads.........-. 17

pipe threads
Form of thread-Continued.

Page

threads of spccial diameters and pitches_ 88

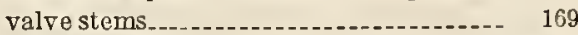

Free fit, class $2 \ldots \ldots \ldots \ldots \ldots \ldots$

Future work of commission.............. 165-169

Gage classification.................. 68, 73, 103, 128

Gages, classification as to accuracy ........... 73, 103 Class X .......................... 73, 75, 105

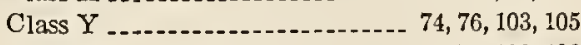

Class Z $Z$....................... 74, 77, 103, 106

design and construction of ......-...... 163, 164 dimensions of

direction of tolerances on .............. 69

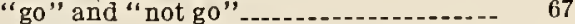

indicating _........... 71

inspection ................... 68, 114, 129, 132, 135

master-.............................. 68, 128, 130

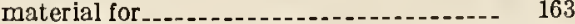

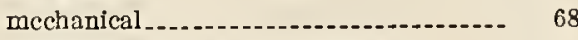

national coarse and fine threads.-.-..-... 67-87

national fire-hose coupling threads..... 113-114

national pipe threads...-...-........... 128-138

threads of special diametcrs and pitches. 103-106

optical_.........- 68

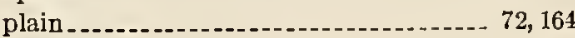

plug thread.............................. 72, 163

ring thread .............................. 71, 164

setting or check.................. 68, 128, 131, 134

specifications for

testing of

thread snap -

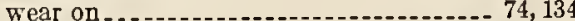

working -...................... 68, 129, 132, 135

Gaging, fundamentals of

ohject of

practices..._._._............. 69-73, 76, 130

Grinding, thread

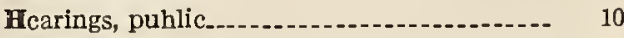

Helix angle_................................ 14

Historical_...

Hoh, threading-............................ 159

Họhs, tooth outlines of -................ 54

Interchangeability

International standardization-_-_-_-_-_.-.-. 11

Lapoing of screw threads................... 162

Lead (definition)

crrors, control of

diametcr equivalent of -.........- 24, $28,32,33,92,112,136,146$

Length of cugagement.-..-..-.-.-.-.-.-14, 22, 116

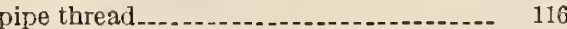

Limits . . . -

Locknut thrcads.

Long screw joints.-_-_..._-_.

Loose fit, class 1........................ 21, 23, 37, 41

Machine screw proportions_...-_-_-_-_-_... 166

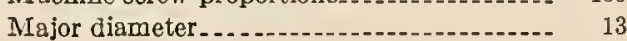


Page

Marking of tools, gages, etc............ 16, 58, 76, 102

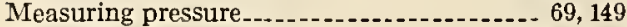

Medium fit, class 3 .................. 22, 30, 39, 43

Members of commission.-................ 7,9

Micrometcrs, thread.................... 70

Minor diameter

uniform, of nut._...................... 22

National coarse-thread series............. 19,

$37-40,45,60-62,65,79,84$

fine-thread series... 20,41-44,49, 61-63, 66, 81, 86

fire-hose coupling threads................ 106-114

form of thread.................... 18, 88, 108

hose-coupling thrcads.-.-.--.-..-.- 106-109, 112

locknut threads

straight pipc threads.

taper pipe threads..............-116-119,137

Net tolerances, basis of ..................... 145

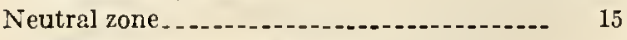

Nomenclature (see Terminology) ..........- 13

Notation _._._._._._. 15-17, 115-116, 120, 123

Number of threads. ........................ 14

Oil-well casing threads........................ 166

Organization of commission . . ............ 6-11

Outside diameter (see Major diameter) .....- 13

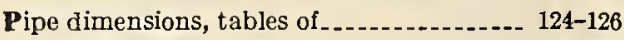
thread gages...-.....-...-...-........... 128-138 manual

threads, straight.-....................... 120-121 tapcr

Pitch........................................ 14

diameter . . . .

measurement of . . . .

uniform, of minimum nut....... 22

Procedure of commission . .................. 10

Public hearings

Report, arrangement of

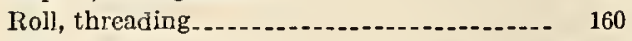

Roller dics. . . . .

Root.

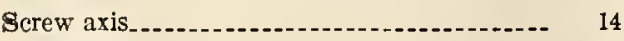

thread................. 13

threads, Acme

cutting of - .

finishing of ....................... 161-162

on oil-well casing.

on tubing . . . 165,169

regular.............. 18-87

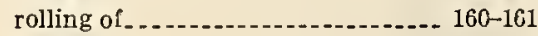

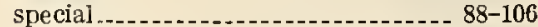

tools for cutting . . .. 53-67, 101-103, 157-159

used in electrical industry . ............ 165

Screws, instrument_....................... 169

machine................ 18-87

wood $\ldots . . . . . . . . . . . . . . . . . . . . . . . . . . . . . .139-144$
Page

of thread ............ 14

Standard temperature......................... 69

Stove bolt proportions.................. 166

Straight pipe threads............. 120-121

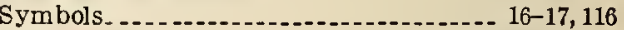

Tap drill sizes................. 63-67, 102-103, 126-127

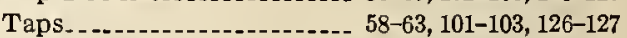
dimensions and tolcrances_60-63, 102-103,126-127 shape of cutting edge for.................- $\quad 59$

Temperature, standard

Terminology . . . .

Thread (see Screw thread)

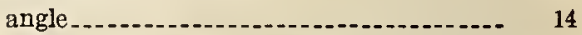
depth (see Dcpth of thread) .............. 14 series...-.......-. 19-21, 88, 108-109, 116-123, 141

Threads, internal and extcrnal............... 13

Threading tools, specifications............ 63-67, $101-103,108,126-128$

Tolerance (dcfinition) . . _.................. 15

Tolerances, derivation of ................. 145-147 direction on gages. 69

hose couplings....................... 110

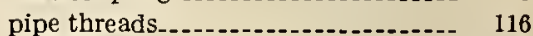

screw and nut........................ 22, 89

(product specifications) ................ 22-23, $88-89,109-112,116,120,122,139-142,169$, gage................... 73-78, 103-106, 130-135 numerical values-

Class 1 , loose fit................. 24, 45-52

Class 2, free fit. .................. 28, 45-52

Class 3 , medium fit............. 32, 45-52

Class 4 , close fit................... 33, 45-52

Class A .

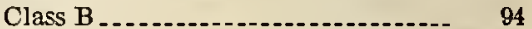

Class C .

Class D .

Class E . .

wood screws.......... 141-142

Tool, single point ... .................... 54, 157-158

Tools, form of . .............. 53-58, 59, 102, 156-162 marking of ........................... 16, 58, 102

Tubing, screw threads on

Uniform minimum nut......................... 22

Utility of report. .

Wire gages.................................. 167-168 methods of measurement. ............. 147-155

Wires, mcasurement of ....... 149-150 sizes of . specification for........................... 149

Wood screws. ................... 139-144

Wrench fit, class 5........................ 22, 167 openings. 






